Emanuela Chiapparini Renate Stohler

Esther Bussmann (Hrsg.)

\title{
Soziale Arbeit \\ im Kontext Schule
}

Aktuelle Entwicklungen in

Praxis und Forschung in der Schweiz 
Soziale Arbeit im Kontext Schule 
Emanuela Chiapparini

Renate Stohler

Esther Bussmann (Hrsg.)

Soziale Arbeit im Kontext Schule

Aktuelle Entwicklungen in Praxis

und Forschung in der Schweiz

Budrich UniPress Ltd.

Opladen • Berlin • Toronto 2018 
Bibliografische Information der Deutschen Nationalbibliothek

Die Deutsche Nationalbibliothek verzeichnet diese Publikation in der Deutschen Nationalbibliografie; detaillierte bibliografische Daten sind im Internet über http://dnb.d-nb.de abrufbar.

\section{Die Publikation konnte dank der finanziellen Unterstützung des Instituts für Kindheit, Jugend und Familie am Departement Soziale Arbeit der Zürcher Hochschule für Angewandte Wissenschaften realisiert werden.}

(C) 2018 Dieses Werk ist bei Verlag Barbara Budrich erschienen und steht unter der Creative Commons Lizenz Attribution-ShareAlike 4.0 International (CC BY-SA 4.0): https://creativecommons.org/licenses/by-sa/4.0/

Diese Lizenz erlaubt die Verbreitung, Speicherung, Vervielfältigung und Bearbeitung bei Verwendung der gleichen CC-BY-SA 4.0-Lizenz und unter Angabe der UrheberInnen, Rechte, Änderungen und verwendeten Lizenz.

\section{(c) (1) (2)}

Dieses Buch steht im OpenAccess Bereich der Verlagsseite zum kostenlosen Download bereit (https://doi.org/10.3224/86388774)

Eine kostenpflichtige Druckversion (Printing on Demand) kann über den Verlag bezogen werden. Die Seitenzahlen in der Druck- und Onlineversion sind identisch.

$\begin{array}{ll}\text { ISBN } & 978-3-86388-774-2 \\ \text { eISBN } & 978-3-86388-328-7 \\ \text { DOI } & 10.3224 / 86388774\end{array}$

Umschlaggestaltung: Bettina Lehfeldt, Kleinmachnow - www.lehfeldtgraphic.de Lektorat: Karina Siuda, Berlin

Satz: Beate Glaubitz, Leverkusen

Druck: Books on Demand GmbH, Norderstedt

Printed in Germany

Verlag Budrich UniPress Ltd. 


\section{Inhalt}

Emanuela Chiapparini, Renate Stohler, Esther Bussmann

Einleitung: Soziale Arbeit im Kontext Schule

I. Grundlagenorientierte Beiträge

Mirjam Staub

„Horte - Verlängerter Arm der Schule oder Unterstützung für

Familien?"

Nina Thieme

Zur Qualität ganztägiger Bildung

Monique Brunner, Roger Pfiffner, Simone Ambord, Ueli Hostettler

Wie Schulsozialarbeit und Schule kooperieren: Fünf Merkmale interdisziplinärer Zusammenarbeit zwischen Schulsozialarbeitenden und Lehrpersonen

Emanuela Chiapparini, Kadrie Selmani, Christa Kappler, Patricia

Schuler

„Die wissen gar nicht, was wir alles machen“. Befunde zu

multiprofessioneller Kooperation im Zuge der Einführung von

Tagesschulen in der Stadt Zürich

Andreas Jud, Madlaina Stauffer \& David Lätsch

Fachliches Handeln an der Schnittstelle von Schule und Kindesschutz: Empirische Erkenntnisse zum Einsatz von Gefährdungsmeldungen in der Schweiz 
II. Perspektiven aus der französischen und deutschen Schweiz ......

Hans-Peter von Aarburg und Jacques Kottelat

Positionierungen der Schulsozialarbeit in der französischsprachigen

Schweiz

Claudia Mühlebach und Marianne Zogmal

Ausserschulische Betreuung in der Romandie - Herausforderungen

und Gedankenanstösse

Anna Maria Riedi und Martin Biebricher

Auf der Suche nach einem gemeinsamen Bildungsverständnis. Ein strukturtypisierender Orientierungsrahmen für die Zusammenarbeit von Schule und Kinder- und Jugendförderung

III. Perspektiven aus der Praxis

Joël Humbert-Droz

„Kann ich mal mit jemandem reden?" Berufsintegration und

Sozialpädagogik in der Schule in Kleingruppen Dielsdorf

Markus Buholzer

Familienklassenzimmer

Marco Dalcher

Sozialpädagogik an der Volksschule Basel-Stadt - Schnittmengen und Stolpersteine

Simon Benz

„Auch wir haben was zu sagen!“ - Beitrag zur Qualitätssteigerung in

Tagesschulen durch Kooperation in interprofessionellen Teams

Aldo Venzi

Schulsozialarbeit und Schulentwicklung - eine Betrachtung aus der Perspektive der Schulsozialarbeit

Karin Vaneck

„Chance und Herausforderung mit Fachpersonen Tagesstrukturen in der Schule zu kooperieren“. Soziale Arbeit in der Schule aus der Sicht einer Schulleiterin

Autorinnen und Autoren 


\title{
Einleitung: Soziale Arbeit im Kontext Schule
}

\author{
Emanuela Chiapparini, Renate Stohler, Esther Bussmann
}

\section{Schule und gesellschaftliche Forderungen}

Über die öffentliche Schule wird in der Schweiz wie auch in anderen Ländern auf unterschiedlichen Ebenen immer wieder kontrovers diskutiert. Zum Beispiel, wenn mit sozialem Wandel einhergehende Herausforderungen und damit verbundene unterschiedliche Lebensführung (Wirth, 2015) zu bewältigen sind (z.B. Wandel der Familienformen, Zuwanderung, Armut, Digitalisierung), wenn sich unerwünschte Folgen des Schulsystems, wie z.B. Bildungsungleichheit (z.B. Becker, Bühler \& Bühler, 2013; Felouzis \& Charmillot, 2017), manifestieren oder wenn Missstände an Schulen publik werden, wie z.B. Cybermobbing unter Schülerinnen und Schülern (Müller et al., 2016). Es wird jeweils die Frage aufgeworfen, ob und welche Aufgaben die Schule zusätzlich übernehmen soll und kann, um Kinder und Jugendliche bestmöglich zu fördern. Kurz: An die Schule werden hohe und komplexe gesellschaftliche Erwartungen gestellt.

Neben Familie und öffentlicher Schule mit ihren vielfältigen Diensten (Baier \& Schnurr, 2008) leistet auch das vielfältige Angebot der Sozialen Arbeit $^{1}$ für Kinder, Jugendliche und Familien (z.B. Kinder- und Jugendarbeit oder Elternbildung, Bundesrat, 2012; Piller \& Schnurr, 2013; Jud et al., 2014) einen wichtigen Beitrag zur Erziehung und Bildung der nachwachsenden Generation.

Kernauftrag der Schule ist der Unterricht in verschiedenen Fächern gemäss Lehrplan (formale Bildung), der von Lehrpersonen mit entsprechender Qualifikation im Klassenverband erteilt wird. Neben der Vermittlung von Unterrichtsstoff (Bildungsauftrag im engeren Sinne) übernehmen Lehrpersonen auch sozialerzieherische Aufgaben im Rahmen der Klassenführung oder

1 In der Grundlagenliteratur steht der Begriff «Soziale Arbeit» in der Regel als Oberbegriff für Sozialarbeit und Sozialpädagogik (z.B. Thole, 2012; Lambers, 2016). Diesem Verständnis folgen grundsätzlich auch die Herausgeberinnen des vorliegenden Buches, wobei der Begriff «Soziale Arbeit» im schweizerischen Kontext neben sozialarbeiterischen und sozialpädagogischen Tätigkeitsbereichen immer auch Tätigkeitsbereiche der soziokulturellen Animation umfasst (Husi \& Villiger, 2012; AvenirSocial, 2014). 
bei der Durchführung von Klassenlagern oder Schulprojekten (Erziehungsauftrag).

Gemäss Scherr (2008) basiert Schule auf einem verkürzten und „funktionalen" Bildungsverständnis, in dem Lehrpersonen die Wissensvermittlung stark in den Vordergrund stellen und das Heranwachsende auf die Berufsbildung und den Arbeitsmarkt vorbereitet, indem Schülerinnen und Schüler Qualifizierungen erwerben. Allerdings ist vor den gegebenen gesellschaftlichen Herausforderungen und im Zuge der Ganztagsbildung vermehrte Persönlichkeitsbildung bzw. „Subjektbildung“ (ebd., S. 139) gefordert, ergänzend zum funktionalen Bildungsverständnis in Schulen. Heranwachsende sollen vermehrt zu eigenverantwortlichen, handlungs-, entscheidungs- und urteilsfähigen Individuen gebildet werden, die keineswegs unabhängig, aber reflexiv soziale Begebenheiten angehen. Offene Lerngelegenheiten und attraktive Angebote für Eigenaktivitäten in der Schule sind anzubieten. Hierbei bietet sich an, dies in der Kooperation mit der Sozialen Arbeit und deren bewährten Theorien und Methoden anzugehen (ebd., S. 144).

\section{Angebote der Sozialen Arbeit im Kontext Schule}

In der deutschsprachigen Schweiz besteht zum Begriff Schulsozialarbeit (SSA) Konsens, dass dieser für ein niederschwelliges, dauerhaftes Angebot der Sozialen Arbeit in den Bereichen Prävention, Beratung und Intervention im Kontext Schule steht (z.B. Ziegele, 2014; Drilling, 2009; AvenirSocial \& SSAV, o.D.; Baier, 2015).

Die SSA wurde in den letzten 25 Jahren in allen deutschsprachigen Kantonen als freiwilliges Angebot an Primar- und/oder Sekundarschulen eingeführt (z.B. Baier, 2011; Müller, 2004; Neuenschwander, Iseli \& Stohler, 2007; Vögeli-Mantovani, 2005) und wird gemäss der neusten Bestandsaufnahme in allen deutschsprachigen Kantonen der Schweiz angeboten, wenn auch der Versorgungsgrad zwischen den Kantonen stark variiert (Seiterle, 2014, S. 87-89). Da in der Schweiz Schul- und Sozialwesen im Kompetenzbereich der Kantone liegen, bestehen in Bezug auf Zielsetzung, inhaltliche Ausrichtung und Organisation der SSA Unterschiede innerhalb und zwischen den Kantonen (vgl. Arnold et al., 2014; Baier, 2015; Ziegele, 2014).

Die ersten Horte als Betreuungsstätten für schulpflichtige Kinder entstanden in der Schweiz Ende des 19. Jahrhunderts innerhalb oder ausserhalb der Schule. Je nach Kanton und Sprachregion entwickelten sich bis heute unterschiedliche Modelle.

Insbesondere in den letzten 15 Jahren wurden in einzelnen Kantonen die Horte in der öffentlichen Schule zu Tagesschulen (z.B. im Kanton Bern, 
Genf, Basel und Zürich) ausgebaut. ${ }^{2}$ Trotz des Versuches, eine einheitliche Definition von Tagesschulen in der Schweiz (Schweizerische Konferenz der kantonalen Erziehungsdirektoren [EDK], 2015) festzuhalten, unterscheiden sich die Tagesschulmodelle in den einzelnen Kantonen bezüglich Teilnahmeverbindlichkeit und pädagogischer Konzepte.

Je nach Tagesschulmodell sind Fachpersonen der Sozialen Arbeit teilweise in einzelnen Unterrichtsstunden (z.B. Klassenrat) und mit unterschiedlichen Funktionen im ausserunterrichtlichen Bereich der Tagesschulen tätig (z.B. Leitung, Betreuung, Förder- und Freizeitangebote). Insbesondere fliessen Konzepte der Sozialen Arbeit (z.B. soziales Lernen oder Partizipation) in die noch weiter auszubauenden Konzepte der Tagesschule ein (z.B. Chiapparini, 2017).

Zudem ist die offene Kinder- und Jugendarbeit in der Schweiz stark verbreitet (Gerodetti \& Schnurr, 2013) und seit geraumer Zeit Kooperationspartnerin der Schule, z.B. bei der Durchführung von Präventionsprojekten (Iseli \& Pfiffner, 2011). Im Zuge der Tagesschulentwicklungen gilt die offene Kinder- und Jugendarbeit als ein wichtiger Drittanbieter für Freizeitangebote in der Schule. Gleichzeitig besteht die Herausforderung darin, die Prinzipien der Freiwilligkeit und Partizipation der Angebote in das Schulsystem einzubinden. Hierzu bestehen einzelne Projekte, z.B. auf der Sekundarstufe in BaselStadt (Erziehungsdepartement des Kantons Basel-Stadt, 2017)

Soziale Arbeit im schulischen Kontext findet aber auch seit längerer Zeit in sozialpädagogisch orientierten Kleingruppen- oder Tagesschulen und Institutionen der stationären Kinder- und Jugendhilfe mit interner Schule statt (Bundesamt für Justiz, 2017) ${ }^{3}$.

\section{Relevanz des vorliegenden Sammelbandes}

Die Sichtung der deutschsprachigen Publikationen in Buchform zum Thema Soziale Arbeit im Kontext Schule macht deutlich, dass Grundlagen und vermehrt auch spezifische Aspekte der Schulsozialarbeit den Schwerpunkt bilden (z.B. Drilling, 2009; Baier \& Deinet, 2011; Baier \& Heeg, 2011; Spies \& Pötter, 2011; Hollenstein \& Nieslony, 2012; Stüwe et al., 2015; Hollenstein et al., 2017).

2 In der Schweiz sind insbesondere Privatschulen und Schulen für Kinder mit besonderen Bedürfnissen als Ganztagsschule organisiert, indem das Mittagessen in der Schule eingenommen wird. Gleichzeitig sind einzelne öffentliche Schulen als Tagesschulen konzipiert, z.B. in der Stadt Zürich sind dies aktuell fünf öffentliche Schulen.

3 Unter https://www.heiminfo.ch kann gezielt nach Heimen mit interner Schule gesucht werden. 
Als ein separates Thema von Sozialer Arbeit im Kontext Schule werden Ganztagsschulen in Deutschland und Tagesschulen in der Deutschschweiz behandelt. In diesen Schulmodellen sind explizit Fachpersonen der Sozialen Arbeit im Handlungsfeld Schule tätig (Speck, Olk \& Stimpel, 2011; Böttcher \& Maykus, 2014). Im Schweizer Kontext liegen noch wenige Studien zur Zusammenarbeit von Fachpersonen der Schulpädagogik und der Sozialen Arbeit vor (Schüpbach et al., 2012; Kappler et al., 2016). Obwohl in Deutschland unvergleichlich viel mehr Studien rund um die Ganztagsschulen zu finden sind, stellt Andresen (2016) fest, dass diese in den letzten 15 Jahren ausschliesslich vom Unterricht her konzipiert sind. Bisher ungenügend wurden beispielsweise die Gestaltungsperspektive der ausserunterrichtlichen Angebote und andere Handlungsfelder der Sozialen Arbeit berücksichtigt, die substantielle Bestandteile von Tagesschulen sind.

Ergänzend zu den skizzierten Buchpublikationen zielt folgender Sammelband darauf $a b$, in der Schweiz aktuelle und ausgewählte Themen im Bereich Schule und Soziale Arbeit aus Forschung und Praxis schlaglichtartig zugänglich zu machen.

Im Besonderen werden erstmals verschiedene gegenwärtige Entwicklungen der Sozialen Arbeit im Kontext Schule in der französischen Schweiz aufgezeigt. Aufgrund des föderalistischen Schulsystems in der Schweiz entstehen vielfältige Möglichkeiten, wie die Angebote der Sozialen Arbeit in der Schule gestaltet und benannt werden (vgl. 2). Vor diesem Hintergrund stellen sich den Herausgeberinnen dieses Sammelbandes nicht nur sprachliche, sondern ebenso begriffliche und konzeptionelle Herausforderungen (vgl. 1).

\section{Kontextualisierung der Beiträge}

Die Beiträge des vorliegenden Sammelbandes beziehen sich auf die Soziale Arbeit im Kontext Schule aus der Praxis, Fachwelt, Bildungsverwaltung und Forschung. Sie lassen sich in folgende drei Gruppen einteilen, welche gleichzeitig die drei Hauptteile des Sammelbandes bilden:

- Grundlagenorientierte Beiträge

- Perspektiven aus der französischen und deutschen Schweiz

- Perspektiven aus der Praxis

In den Beiträgen dieses Sammelbandes verwenden die Autorinnen und Autoren die Begriffe Soziale Arbeit, Sozialpädagogik, Sozialarbeit und soziokulturelle Animation sowie die Bezeichnungen für die entsprechenden Fachpersonen (vgl. Kap. 1) teilweise unterschiedlich, insbesondere dort, wo sie im 
Praxisfeld oder Fachdiskurs so üblich sind. Die Herausgeberinnen des Sammelbandes haben diese Begriffsvielfalt bewusst so belassen.

Im ersten Teil „Grundlagenorientierte Beiträge“ wirft Mirjam Staub mit ihrem Beitrag einen historischen Blick auf die Soziale Arbeit im Kontext Schule, indem sie die Anfänge der Hortentwicklung in der Deutschschweiz und exemplarisch die Hortentwicklung in den Kantonen Zürich und St. Gallen Ende des 19. Jahrhunderts aufzeigt. Der Auftrag der Horte hat sich im Laufe der Zeit gewandelt, wie die Autorin in ihrem Beitrag detailliert dokumentiert. Gleichzeitig steht dieser bis heute im Spannungsfeld zwischen Schule und Sozialer Arbeit sowie zwischen Schule und Familie. Vor diesem Hintergrund plädiert die Autorin für eine verstärkte interprofessionelle Zusammenarbeit der Fachpersonen der Sozialen Arbeit.

Ein Dauerbrenner in der Bildungsforschung ist die Qualitätssicherung, welche konträr diskutiert wird. Im Zusammenhang mit Ganztagsschulen (vgl. bspw. Sauerwein und Klieme, 2016) steht diese zudem vermehrt in Diskussion (Fischer et al., 2016; Thieme, 2016) und auch in der Deutschschweiz werden Qualitätskriterien für Tagesschulen entwickelt und gefordert (Brückel et al., 2017).

Hierzu bietet Nina Thieme in ihrem Beitrag theoriebasierte Reflexionen zum Qualitätsverständnis ganztägiger Bildung. In Abgrenzung zum verbreiteten objektiven Qualitätsbegriff votiert sie für einen relationalen Begriff von Qualität, indem sie von Aushandlungsmomenten zwischen schulischen und sozialpädagogischen Fachpersonen und dem ungleichen Verhältnis zwischen Lehrpersonen und Schülerinnen und Schülern ausgeht. Vor diesem Hintergrund stellt sich gemäss der Autorin weniger die Frage, was als Qualität gilt, sondern vielmehr die Frage, inwiefern die strukturellen Bedingungen für eine aushandlungsorientierte Kinder- und Jugendarbeit gegeben sind, damit alle Beteiligten sich gleichberechtigt einbringen können.

Zur Zusammenarbeit und zu gegenseitigen Erwartungen von Lehrpersonen und Schulsozialarbeitenden liegen primär im Rahmen von Evaluationen erhobene Befunde vor (Baier \& Heeg, 2011; Vogel, 2013), welche Aussagen dazu machen, wie sich diese ausgestalten und wo Chancen und Herausforderungen liegen. Es zeichnet sich bezüglich der Schulsozialarbeit ab, dass sich die Daten vornehmlich auf die Deutschschweiz beziehen. Zur Schliessung dieser Wissenslücke leistet das vom Schweizerischen Nationalfonds geförderte Forschungsprojekt „Kooperationsformen und Nutzungsstrukturen in der Schulsozialarbeit" einen Beitrag, in dem in 17 Kantonen in drei Sprachregionen der Schweiz Schulsozialarbeitende, Schulleitende und Lehrpersonen anhand von Fragenbögen zu ihrer Kooperationstätigkeit befragt werden. Aus dieser Studie stellen Monique Brunner, Roger Pfiffner, Simone Ambord und 
Ueli Hostettler in ihrem Beitrag erste Befunde vor, wie Schulsozialarbeit und Lehrpersonen zusammen kooperieren. Dies wird anhand der fünf Merkmale interdisziplinärer Zusammenarbeit nach Bronstein (2003) vorgenommen: Interdependenz, Gemeinsame Ziele, Flexibilität, Neue Aktivitäten, Reflexion Arbeitsprozess.

Die Zusammenarbeit zwischen Lehrkräften und sozialpädagogischen Fachkräften spielt ebenfalls in der Einführung von Tagesschulen eine zentrale Rolle und gilt hierzu als ein wichtiges Qualitätsmerkmal (Olk et al., 2011; Fischer, Klieme, Holtappels, Stecher \& Rauschenbach, 2013). Gleichzeitig besteht wenig Wissen über die Gestaltung der Zusammenarbeit zwischen Lehrkräften und sozialpädagogischen Fachkräften und darüber, wie beide Berufsgruppen sich gegenseitig wahrnehmen. Darauf geht der Beitrag von Emanuela Chiapparini, Kadrie Selmani, Christa Kappler und Patricia Schuler ein, in welchem hierzu erste Befunde aus der vom Schweizerischen Nationalfonds geförderten qualitativen Studie zu pädagogischen Zuständigkeiten im Zuge der Einführung von Tagesschulen in der Stadt Zürich (,AusTEr“) vorgestellt und diskutiert werden.

Ein weiteres zentrales Thema der Sozialen Arbeit in der Schule sind Gefährdungsmeldungen im Rahmen des zivilrechtlichen Kinderschutzes. Die Entscheidung für oder gegen eine Gefährdungsmeldung ist von einem Dilemma geprägt: Einerseits kann der nötige Kinderschutz aufgrund einer zu späten oder unterlassenen Gefährdungsmeldung nicht geleistet werden, andererseits besteht die Gefahr, unnötigerweise die Privatsphäre der Familie zu beinträchtigen. Mit diesen Unsicherheiten treffen Schulleitungen, Lehrpersonen und Schulsozialarbeitende einschneidende Entscheidungen für das Kind resp. die Familie. Hierzu bietet eine vom Schweizerischen Nationalfonds unterstützte Studie Erkenntnisse zu den auslösenden und hindernden Faktoren für eine Gefährdungsmeldung in der Deutschschweiz. Im Mittelpunkt des Beitrags von Andreas Jud, Madlaina Stauffer und David Lätsch stehen Antworten auf die Frage, nach welchen Überlegungen sich Lehrpersonen, Schulleitende und Schulsozialarbeitende für oder gegen eine Gefährdungsmeldung entscheiden. Die Befunde weisen darauf hin, dass diese Entscheidung stark von der Kooperationsbereitschaft der Eltern abhängig ist.

Im zweiten Teil des Sammelbandes werden zu drei Themenfeldern der Sozialen Arbeit im Kontext Schule - Schulsozialarbeit, ausserschulische Betreuung und Kinder- und Jugendförderung - erstmals Entwicklungen aus der französischen Schweiz mitberücksichtigt.

Während sich die Schulsozialarbeit in den letzten zwanzig Jahren in der Deutschschweiz etabliert hat und sich gemäss Baier und Deinet (2011) in der Profilierungsphase befindet, entwickelten sich in der französischen Schweiz 
verschiedene Modelle der Kooperation zwischen Schule und Sozialer Arbeit mit unterschiedlichen Bezeichnungen. Im Übersichtsbeitrag von Hans-Peter von Aarburg und Jacques Kottelat werden die unterschiedlichen Formen schulischer Sozialarbeit in der französischsprachigen Schweiz systematisch vorgestellt und diskutiert. Zudem werden Antworten gegeben, wie ein Nebeneinander von verschiedenen Formen der Sozialen Arbeit in der Schule möglich ist.

Im Zuge der Entwicklung der Ganztagsbildung im Kontext Schule, für welche in der Deutschschweiz die Begriffe Tagesschule und Tagesstruktur bezeichnend sind, etabliert sich der parallele Begriff der „école à l'horaire continu“ (EDK, 2017) nicht in gleicher Weise. Ganztägige Bildungsangebote sind hingegen unter dem Begriff der „Acceuil parascolaire“ (ausserschulische Angebote) wiederzufinden (ebd.). Während in der Stadt Genf ein gemeindeübergreifendes Angebot vorliegt, sieht dies im Kanton Waadt viel heterogener aus. Anhand dieser konträren Beispiele stellen Claudia Mühlebach und Marianne Zogmal die ausserschulischen Betreuungsangebote im Kontext Schule vor, indem sie die Entstehungsgeschichte, die Ziele, Qualität und die qualifizierten Fachpersonen in den beiden französischsprachigen Kantonen diskutieren.

In den letzten Jahren hat die Offene Kinder- und Jugendarbeit in der Schweiz eine zunehmende Aufbau- und Strukturentwicklung auf kommunaler, kantonaler sowie nationaler Ebene erfahren (Gerodetti und Schnurr, 2013). Insbesondere die Offene Kinder- und Jugendarbeit wird im Rahmen der Ganztagsbildung als attraktive und potentielle Kooperationspartnerin für die Schule bewertet. Vor dem Hintergrund der vergleichbaren Angebote in der italienischsprachigen, französischen und deutschsprachigen Schweiz, welche erstmals systematisch in den kommenden Jahren untersucht werden (Dachverband offene Kinder- und Jugendarbeit Schweiz (DOJ), 2017), bietet der Beitrag von Anna Maria Riedi und Martin Biebricher für den sprachübergreifenden Diskurs einen strukturtypisierenden Orientierungsrahmen für die Zusammenarbeit zwischen Schule und Kinder- und Jugendförderung. Hierbei geht es wesentlich um das Verständnis eines gemeinsamen Bildungsbegriffs zwischen den beteiligten Fachpersonen der Sozialen Arbeit und der Schule.

Im dritten Teil des Sammelbandes wird die Soziale Arbeit im Kontext Schule aus der Perspektive der Praxis mit ausgewählten Beiträgen vertieft.

Der Sozialen Arbeit kommt im Rahmen der Schulen in Kleingruppen eine zentrale Rolle bezüglich Berufsintegration und sozialpädagogischer Begleitung im Unterricht zu. Joël Humbert-Droz, Sozialpädagoge, gibt in seinem Beitrag einen Einblick in die Schule in Kleingruppen Dielsdorf, indem 
er auf konzeptioneller und erfahrungsbasierter Ebene die zentrale Rolle der Sozialpädagoginnen und Sozialpädagogen in der Schule in Kleingruppen vorstellt und diskutiert. Hierbei sind klare Verantwortungsbereiche im Team, wöchentliche Austauschsitzungen mit Lehrpersonen und Erhöhung der Stellenprozente für die Sozialpädagoginnen und Sozialpädagogen Kernelemente der optimalen schulischen Bildung von Lernenden.

In den letzten zehn Jahren bewährte sich in den Primarschulen Kriens das Konzept der Familienklassenzimmer, um komplexe Problemsituationen eines Kindes zusammen mit der Familie, den Lehrpersonen und Fachpersonen der Sozialen Arbeit in einem gemeinsamen Lernsetting innerhalb der Schule anzugehen. Der Schuldirektor der öffentlichen Schulen in Kriens, Markus Buholzer, stellt das Konzept anhand der zentralen systemtheoretischen Elemente, basierend auf Erfahrungsbeispielen und Befunden aus Evaluationen, vor und diskutiert dieses.

Am Beispiel Tagesstrukturen auf der Sekundarstufe in Basel-Stadt zeigt Marco Dalcher in seinem Beitrag zur Sozialpädagogik an der Volksschule Basel-Stadt auf, wie Schulpädagogik und Sozialpädagogik Hand in Hand zum Wohlbefinden von Kindern und Jugendlichen beitragen können und sollen. Schule wird von den Jugendlichen nicht nur als Ort der formalen Bildung, sondern beispielsweise auch als Aufenthaltsort wahrgenommen. Ausgehend von jugendgerechten Angeboten der Tagesstruktur an der Volksschule Basel-Stadt diskutiert der Autor die Chancen und Risiken einer Verschränkung von zwei Disziplinen.

Simon Benz, angehende Fachperson Sozialer Arbeit, plädiert in seinem Beitrag für bessere Qualität in Tagesschulen durch Kooperation in interprofessionellen Teams und weist damit auf die Potentiale der Zusammenarbeit von Lehrpersonen und Fachpersonen Sozialer Arbeit in Tagesschulen hin. Ausgehend von empirischen Befunden zeigt der Autor exemplarisch an einem Hortkonzept einer Tagesschule auf, wie sich die Zusammenarbeit zwischen Fachpersonen der Sozialen Arbeit und Lehrpersonen in der Praxis gestalten kann.

Aldo Venzi geht in seinem Beitrag der Frage nach, ob und inwiefern die Schulsozialarbeit die Entwicklungsprozesse einer Schule beeinflussen soll oder kann. Ausgehend von Befunden aus Deutschland, welche auf der Ebene der Schulkultur und des Schulklimas eine Beeinflussungsmöglichkeit der Schulsozialarbeit belegen, veranschaulicht dies der Autor am Fallbeispiel der Einführung von Konfliktlotsen in einem Schulhaus. Hierzu werden hemmende und fördernde Aspekte der Schulsozialarbeit bezüglich Schulentwicklung reflektiert.

Am Beispiel des sozialen Brennpunktquartiers Thierstein in Basel-Stadt, in welchem 2012 das Projekt der schulzentrierten Bildungslandschaften ge- 
startet ist, stellt Karin Vaneck, Schulleiterin in jenem Quartier, die Rolle der Sozialen Arbeit im Kontext Schule aus ihrer Perspektive vor. Fachpersonen der Sozialen Arbeit sind im Unterricht tätig. Zudem geht das Konzept der Bildungslandschaft von den bestehenden sozialpädagogischen Angeboten im Quartier aus. Die Zusammenarbeit der Fachpersonen Sozialer Arbeit, Lehrpersonen und weiterer Fachpersonen in den Tagesstrukturen, im Unterricht und in den sozialpädagogischen Angeboten im Quartier (Drittanbietende) wird vorgestellt und diskutiert.

\section{Literatur}

Andresen, S. (2016). Lern- und Bildungsgelegenheiten in der Ganztagsschule. Die Erweiterung der Perspektive über den Unterricht hinaus. Zeitschrift für Pädagogik, 62(6).

Arnold, R., Brandstetter, J., Eugster, R., Müller, M. \& Reutlinger, C. (2014). 8 x Schulsozialarbeit. Acht Berichte und Reflexionen aus einer vielfältigen Praxis. Berlin: Frank \& Timme GmbH.

AvenirSocial (2014). Berufsbild der Professionellen Sozialer Arbeit. Bern: AvenirSocial. Retrieved from:

http://www.avenirsocial.ch/cm_data/AS_Berufsbild_DE_def_1.pdf [Zugriff: 25. 02. 2018].

AvenirSocial \& SSAV (o.D.). Leitbild Soziale Arbeit in der Schule. Online verfügbar unter: http://www.avenirsocial.ch/cm_data/AS_DE_Schulsozarbeit_160329.pdf [Zugriff: 25.02.2018].

Baier, F. (2011). Schulsozialarbeit in der Schweiz. In: F. Baier \& U. Deinet (Hg.), Praxisbuch Schulsozialarbeit. Methoden, Haltungen und Handlungsorientierungen für eine professionelle Praxis (S. 57-84). Opladen: Budrich.

Baier, F. (2015). Schulsozialarbeit in der Schweiz. In: T. Olk \& K. Speck (Hg.), Reader Schulsozialarbeit Band 3: Von den Nachbarn lernen - Internationaler Vergleich von Jugendsozialarbeit an Schule. Bd. 3. Berlin: Eigenverlag Deutsches Rotes Kreuz e.V., S. 41-61.

Baier, F. \& Deinet, U. (Hg.) (2011). Praxisbuch Schulsozialarbeit. Methoden, Haltungen und Handlungsorientierungen für eine professionelle Praxis. Opladen: Budrich.

Baier, F. \& Heeg, R. (2011). Praxis und Evaluation von Schulsozialarbeit. Sekundäranalysen von Forschungsdaten aus der Schweiz. Wiesbaden: VS Verlag.

Baier, F. \& Schnurr, S. (Hg.) (2008). Schulische und schulnahe Dienste. Angebote, Praxis und fachliche Perspektiven. Bern: Haupt.

Becker, R., Bühler, P. \& Bühler, T. (Eds.) (2013). Bildungsungleichheit und Gerechtigkeit: Wissenschaftliche und gesellschaftliche Herausforderungen. Prisma: Beiträge zur Erziehungswissenschaft aus historischer, psychologischer und soziologischer Perspektive. Bd. 20. Bern: Haupt Verlag. 
Böttcher, W. \& Maykus, S. (2014). Sozialpädagogin und Sozialpädagoge sein in der Ganztagsschule. In: S. Maschke, G. Schulz-Gade \& L. Stecher (Hg.), Jahrbuch Ganztagsschule 2014. Inklusion. Der pädagogische Umgang mit Heterogenität. Schwalbach/Ts.: Debus Pädagogik, S. 88-101.

Bronstein, L. (2003). A model for interdisciplinary collaboration. Social Work, 48(3), S. 297-306.

Brückel, F., Kuster, R., Annen, L. \& Larcher S. (Hg.) (2017). Qualität in Tagesschulen/Tagesstrukturen (QuinTaS). Bern: hep.

Bundesamt für Justiz (2017): Verzeichnis der vom Bundesamt für Justiz anerkannten Erziehungseinrichtungen. Stand 07.11.2017. Online verfügbar unter: https://www.bj.admin.ch/dam/data/bj/sicherheit/smv/dokumentation/heimverzeic hnis-d.pdf [Zugriff: 25.02.2018].

Bundesrat (2012). Gewalt und Vernachlässigung in der Familie: notwendige Massnahmen im Bereich der Kinder- und Jugendhilfe und der staatlichen Sanktionierung. Bericht des Bundesrates in Erfüllung des Postulates Fehr (07.3725) vom 5. Oktober 2007. Online verfügbar unter:

https://www.bsv.admin.ch/bsv/de/home/sozialpolitische-themen/kinder-undjugendfragen/Kinderschutz/gewalt-und-vernachlaessigung-in-der-familie.html [Zugriff: 31.01.2018].

Chiapparini, E. (2017). Förderung des Wohlbefindens von Kindern durch die pädagogische Arbeit der Lehrkräfte und Fachpersonen Tagesstrukturen. Zwei Literaturarbeiten und eine empirische Untersuchung auf Kindergartenstufe in der Stadt Basel. Zürich: Zürcher Hochschule für Angewandte Wissenschaften. Online verfügbar unter:

https://www.zhaw.ch/storage/shared/sozialearbeit/Forschung/Kindheit_Jugend_F amilie/Soziale_Arbeit_Schule/f\%C3\%B6rderung-wohlbefinden-kinderchiapparini-emanuela.pdf [Zugriff: 24.04.2017].

Dachverband offene Kinder- und Jugendarbeit Schweiz (DOJ) (2017). Projektskizze Projekt „Offene Kinder- und Jugendarbeit - Travail avec les enfants et des jeunes - Animazione socioculturale dell'infanzia e della gioventù in allen Sprachregionen der Schweiz“. Unter Mitarbeit von M. Casutt. Bern.

Drilling, M. (2009). Schulsozialarbeit: Antworten auf veränderte Lebenswelten (4., aktualisierte Aufl. ed.). Bern: Haupt.

Erziehungsdepartement des Kantons Basel-Stadt, Volksschule. (2017). Konzept für Hausaufgabenunterstützung (HAU): Sekundarschule Drei Linden. Tagesstruktur.

Fischer, N., Klieme, E., Holtappels, H.-G., Stecher, L. \& Rauschenbach, T. (2013). Ganztagsschule 2012/2013. Deskriptive Befunde einer bundesweiten Befragung. Ergebnisse der Schulleitungsbefragung. Frankfurt a.M., Dortmund, Gießen \& München.: StEG 2012.

Fischer, N., Kuhn, H. P. \& Tillack, C. (Hg.) (2016). Was sind gute Schulen? Teil 4: Theorie, Praxis und Forschung zur Qualität von Ganztagsschulen. Immenhausen: Prolog.

Gerodetti, J. \& Schnurr, S. (2013). Offene Kinder- und Jugendarbeit in der Schweiz. In: U. Deinet \& B. Sturzenhecker (Hg.), Handbuch Offene Kinder- und Jugendarbeit. 4., überarbeitete und aktualisierte Aufl. Wiesbaden: Springer VS, S. 827-839. 
Hollenstein, E. \& Nieslony, F. (Hg.) (2012). Handlungsfeld Schulsozialarbeit. Profession und Qualität. Hohengehren: Schneider.

Hollenstein, E., Nieslony, F., Speck, K. \& Olk, T. (Hg.) (2017). Handbuch der Schulsozialarbeit (Band 1). Weinheim Basel: Beltz.

Husi. K. \& Villiger, S. (2012). Sozialarbeit, Sozialpädagogik, Soziokulturelle Animation. Theoretische Reflexionen und Forschungsergebnisse zur Differenzierung Sozialer Arbeit. Luzern: interact.

Iseli, D. \& Pfiffner, R. (2011). Zusammenarbeit und Koordination Offene Kinder- und Jugendarbeit und Schulsozialarbeit. Bericht. Bern: Berner Fachhochschule.

Jud, A., Fergert, J. M. \& Schlup, M. (Hg.) (2014). Kinder- und Jugendhilfe im Trend. Veränderungen im Umfeld der Kinder- und Jugendhilfe am Beispiel der Stadt Zürich. Luzern: interact.

Kappler, C., Chiapparini, E. \& Schuler, P. (2016). Die gute neue Tagesschule in der Schweiz. Der Erziehungs- und Bildungsauftrag aus der Sicht der Professionen. Schulpädagogik - heute, 7(13), S. 1-15.

Lambers, Helmut (2016). Theorien der Sozialen Arbeit. Ein Kompendium und Vergleich. Opladen \& Toronto: Verlag Barbara Budrich.

Müller, S. (2004). Schulsozialarbeit im Kanton Zürich. Schlussbericht. Fachhochschule Zürich. Hochschule für Soziale Arbeit. Zürich.

Neuenschwander, P., Iseli, D. \& Stohler, R. (2007). Bestandesaufnahme der Schulsozialarbeit im Kanton Bern. Bern: BFH Soziale Arbeit.

Olk, T., Speck, K. \& Stimpel, T. (2011). Professionelle Kooperation unterschiedlicher Berufskulturen an Ganztagsschulen - Zentrale Befunde eines qualitativen Forschungsprojektes. In: L. Stecher, H.-H. Krüger \& T. Rauschenbach (Hg.), Ganztagsschule. Neue Schule? Wiesbaden: VS Verlag, S. 63-80.

Piller, E. \& Schnurr, S. (Hg.) (2013). Kinder- und Jugendhilfe in der Schweiz. Forschung und Diskurse. Wiesbaden: Springer VS.

Sauerwein, M. \& Klieme, E. (2016). Anmerkungen zum Qualitätsbegriff in der Bildungsforschung. Schweizerische Zeitschrift für Bildungsforschung, 38(3), S. 459-478.

Schüpbach, M., Jutzi, M. \& Thomann, K. (2012). Expertise zur Kooperation in verschiedenen Kooperationsfeldern. Eine qualitative Studie zu den Bedingungen gelingender multiprofessioneller Kooperationen in zehn Tagesschulen. Zuhanden des Erziehungsdepartements. Bern: Universität Bern, Institut für Erziehungswissenschaft.

Seiterle, N. (2014). Schulsozialarbeit in der Deutschschweiz. Eine Standortbestimmung. In: K. Gschwind (Hg.), Soziale Arbeit in der Schule. Definition und Standortbestimmung. Luzern: interact, S. 82-153.

Speck, K., Olk, T. \& Stimpel, T. (2011). Auf dem Weg zu multiprofessionellen Organisationen? Die Kooperation von Sozialpädagogen und Lehrkräften im schulischen Ganztag. Empirische Befunde aus der Ganztagsforschung und dem Forschungsprojekt „Professionelle Kooperation von unterschiedlichen Berufskulturen an Ganztagsschulen“ (ProKoop). In: W. Helsper \& R. Tippelt (Hg.), Pädagogische Professionalität. Weinheim: Beltz, S. 184-201. 
Spies, A. \& Pötter, N. (2011). Soziale Arbeit an Schulen. Einführung in das Handlungsfeld Schulsozialarbeit. 1. Aufl. Wiesbaden: VS Verlag für Sozialwissenschaften.

Stüwe, G., Ermel, N. \& Haupt, S. (2015). Lehrbuch Schulsozialarbeit. Weinheim und Basel: Beltz.

Thieme, N. (2016). (Mehr) Qualität des Bildungssystems durch Ganztagesschulen mit Qualität? Theoretische Überlegungen und rekonstruktive Vergewisserungen zu den Voraussetzungen, um über Qualität verhandeln zu können. In: S. Blömeke, M. Caruso, S. Reh, U. Salaschek \& J. Stiller (Hg.), Tradition und Zukünfte. Beiträge zum 24. Kongress der Deutschen Gesellschaft für Erziehungswissenschaft. Opladen, Berlin \& Toronto: Budrich, S. 245-256.

Thole, W. (Ed.) (2012). Grundriss Soziale Arbeit: Ein einführendes Handbuch (4. Aufl.). Wiesbaden: VS Verlag für Sozialwissenschaften. Retrieved from: http://sfx.metabib.ch/sfx locater?sid=ALEPH: EBI01\&genre =book\&isbn=9783-531-94311-4 [Zugriff: 09.03.2018].

Vogel, Ch. (2013). Mythos Kooperation. Die Klischierung des Legitimationsproblems in aktuellen Institutionalisierungsformen der Schulsozialarbeit. In: E. Piller \& S. Schnurr (Hg.), Kinder- und Jugendhilfe in der Schweiz. Forschung und Diskurse. Wiesbaden: Springer, S. 197-228.

Vögeli-Mantovani, U. (2005). Die Schulsozialarbeit kommt an! Aarau: Schweizerische Koordinationsstelle für Bildungsforschung.

Wirth, J. V. (2015). Die Lebensführung der Gesellschaft: Grundriss einer allgemeinen Theorie. Wiesbaden: Springer. Retrieved from: http://sfx.ethz.ch/sfx locater?sid=ALEPH:EBI01\&genre=book\&isbn=97836580 77068 [Zugriff: 09.03.2018].

Ziegele, U. (2014). Soziale Arbeit in der Schule. In: K. Gschwind (Hg.), Soziale Arbeit in der Schule. Definition und Standortbestimmung. Luzern: interact, S. 1478.

Ziegele, U., Gschwind, K. \& Seiterle, N. (2014). Soziale Arbeit in der Schule. Definition und Standortbestimmung. Luzern: interact, Hochschule Luzern. 


\section{Grundlagenorientierte Beiträge}





\title{
„Horte - Verlängerter Arm der Schule oder Unterstützung für Familien?““
}

\author{
Mirjam Staub
}

„Entwicklungen im Arbeitsmarkt“, „vermehrte Berufstätigkeit der Frauen“, „gewandeltes Verständnis der Rollen von Mann und Frau in Familie und Kindererziehung“ (EDK, 2011, S. 27). Diese Schlagworte werden im HarmoSKonkordat der schweizerischen Erziehungsdirektorenkonferenz als Begründung aufgeführt, weshalb heute vermehrt Betreuungsangebote für Schülerinnen und Schüler geschaffen werden sollen. Das Thema der ausserschulischen Betreuung von Schulkindern wird heute, unter anderem dank dem Konkordat, denn auch vermehrt auf die politische Agenda gesetzt. Auffallend ist aber, dass die Wichtigkeit von Betreuungsangeboten zwar mit gesellschaftlichen Veränderungen begründet wird, es offensichtlich aber bis heute nicht genügt, die Kinder lediglich zu beaufsichtigen, um den Eltern zu ermöglichen, einer Arbeit ausser Haus nachzugehen. Vielmehr werden Tagesstrukturen mit verschiedenen pädagogischen Absichten verbunden, deren Qualitätsmerkmale und Wirksamkeit jedoch nach wie vor Gegenstand der Schulforschung sind (Schüpbach, Frei \& Nieuwenboom, 2017). Ausserdem werden Tagesstrukturen bis heute sehr unterschiedlich organisiert und geführt. Der Grund für diese Verschiedenheit in Trägerschaft, Personal und pädagogischer Konzeption liegt allerdings nicht nur in den föderalistischen Strukturen der Schweiz, sondern ist vielmehr historisch gewachsen. Auffallend ist jedoch, dass Forschung und Diskurs rund um Tagesstrukturen bzw. um Betreuungsangebote für Schulkinder bisher mehrheitlich ahistorisch geführt werden. Die aktuelle Entwicklung wird meistens lediglich aus der jüngeren Geschichte der wenigen Tagesschulversuche in der Deutschschweiz oder der Einführung der Blockzeiten zur Unterrichtsorganisation hergeleitet (Grunder, 2008; Schüpbach, Frei \& Nieuwenboom, 2017). Für die Entstehung der Tagesstrukturen ${ }^{1}$, unter welche auch die Betreuung von

1 Der Begriff „,Tagesstrukturen“ umfasst sämtliche Betreuungsangebote für Kinder und Jugendliche ausserhalb der Familie, wobei unterschieden wird zwischen modularen Tagesstrukturen, in denen Betreuung durch die Schule oder andere Anbieter vor, zwischen und nach dem Unterricht angeboten werden und individuell in Anspruch genommen werden 
Kindern in Horten fällt, gibt es bisher keine umfassenden historischen Untersuchungen, obwohl die ersten Horte für Schulkinder bereits Ende des 19. Jahrhunderts gegründet wurden. Diesem Desiderat nimmt sich der folgende Beitrag an, dem die Ergebnisse meines Dissertationsprojektes zugrunde liegen, welches die Erforschung der Anfänge und der Institutionalisierung der ausserschulischen Betreuung von Schulkindern in der Deutschschweiz im 19. und 20. Jahrhundert zum Ziel hat. ${ }^{2}$ Am Beispiel der ersten Horte in der Deutschschweiz, die in den Städten Zürich und St. Gallen um 1886 entstanden sind ${ }^{3}$ und die als Pionierwerke gelten können, wird im Folgenden erläutert, wie sich diese im Spannungsfeld zwischen Schule und Familie entwickelten und welche Rolle die sich um die Jahrhundertwende zu etablieren beginnende Soziale Arbeit im Kontext der Betreuung von Schulkindern spielte.

\section{Pädagogisches Konzept - schulnah oder familienergänzend?}

In der Stadt Zürich wurde der erste Hort für Knaben 1886, der erste Hort für Mädchen fünf Jahre später gegründet. 1887 entstanden der erste Knaben- und der erste Mädchenhort in der Stadt St. Gallen. In beiden Städten ging die Initiative zwar von Einzelpersonen aus, diese brachten ihr Anliegen jedoch vor die Schulbehörden, die Lehrerschaft und vor gemeinnützige Kreise, aus denen je eine eigens zum Zweck der Führung der Horte eingesetzte Kommission gebildet wurde. Für die Initianten an beiden Orten war klar, dass eine solche Neugründung ein Werk der privaten Gemeinnützigkeit sein müsse und diese Aufgaben nicht der Schule bzw. der öffentlichen Hand übertragen werden konnte. Dennoch waren sie auf die Zusammenarbeit mit der Schule und den Schulbehörden angewiesen. Die Horte in Zürich und St. Gallen wurden

können, sowie gebundenen Tagesstrukturen, in denen die Kinder verbindlich das Betreuungsangebot, das ganztägig, ausserhalb der Unterrichtszeiten, jedoch durch die Schule (z.B. Tagesschule) angeboten wird, besuchen (BFS, 2015).

2 In meinem Dissertationsprojekt zeichne ich, unter Rückgriff auf den Theorieansatz des Akteurzentrierten Institutionalismus den Institutionalisierungsprozess der Horte für Schulkinder im 19. und 20. Jahrhundert nach. Als Quellenkorpus dienen Jahresberichte und Sitzungsprotokolle der Kinderhortkommissionen sowie Tagebücher und Berichte der Hortleitenden. (siehe auch:

https://www.uzh.ch/blog/ife-hbs/forschungsprojekte/laufend/kinderhorte)

3 Der erste Hort für Schulkinder in der Schweiz wurde 1886 in Winterthur gegründet. Allerdings ist hierzu die Quellenlage dürftig. Nach den Gründungen in Zürich 1886 und St. Gallen 1887 entstanden weitere ähnliche Einrichtungen in Basel, Bern, Lausanne, Vevey und Genf (Fisler, 1898). 
durch die Schulbehörden finanziell unterstützt und fanden meist in unentgeltlich zur Verfügung gestellten Schulzimmern statt.

Während in Zürich die Verflechtung der Kinderhortkommission mit der Schule von Anfang an sehr eng war, da deren erster Präsident Paul Hirzel gleichzeitig Präsident der Stadtschulpflege war, organisierte sich die Kommission in St. Gallen von den Schulbehörden und der Lehrerschaft unabhängiger. Sie publizierte beispielsweise die Aufrufe zur Anmeldung von Kindern in die Horte in den Tageszeitungen und sprach damit die Eltern der Kinder direkt an, anstatt, wie es die Kommission in Zürich handhabte, die Lehrpersonen dazu aufzurufen, Kinder zu melden, deren „ökonomische Bedürftigkeit“" und „Grad der Aufsichtslosigkeit“ (Jahresbericht Zürich, 1887, S. 7) für eine Aufnahme in den Hort sprach. Die Horte in Zürich und St. Gallen richteten sich dennoch in erster Linie an Eltern, die sich aufgrund ihrer Arbeitstätigkeit nach Unterrichtsschluss nicht um ihre Kinder kümmern konnten. Beide Kinderhortkommissionen betonten in den 1880er Jahren, dass die Notwendigkeit zur Schaffung von Horten darin begründet liege, dass die schwierige Erwerbssituation, bedingt durch die Industrialisierung, dazu führe, dass auch viele Mütter einer Erwerbsarbeit ausserhalb des Hauses nachgehen müssten. Die zeitliche Abwesenheit der Mütter galt als Ursache der Verwahrlosung von Kindern, entsprechend wurden die Horte als Beitrag zur Lösung der Ende des 19. Jahrhunderts viel diskutierten „Sozialen Frage“ dargestellt.

In den Horten sollte dieser Mangel an Beaufsichtigung und Erziehung ausgeglichen werden und weil sie die Zeiten umfassten, in denen eigentlich die Eltern für diese Aufgaben zuständig wären, sollten sie folglich das Familienleben und die Familienerziehung temporär ersetzen (Jahresbericht Zürich, 1887, S. 1; Jahresbericht St. Gallen, 1887, S. 4). Das pädagogische Konzept, das daraus abgeleitet wurde, sah sowohl in Zürich als auch in St. Gallen vor, dass die Gestaltung der Horte möglichst familienähnlich sein sollte. Entgegen der seit 1872 existierenden Vorbilder aus Deutschland (Fisler, 1898; Rolle \& Kessberg, 1988) wurden in den Horten in Zürich und St. Gallen daher eher kleinere Gruppen von 20 bis 25 Kindern im schulpflichtigen Alter geschlechtergetrennt betreut. Die Kinder besuchten den Hort jeweils am Nachmittag nach Unterrichtsschluss. Im Hort erhielten sie zuerst einen kleinen Imbiss bestehend aus Brot und Milch, danach wurden die Hausaufgaben erledigt und die verbleibende Zeit mit „sinnvoller Beschäftigung“ gefüllt. Dies hiess etwa, dass mit den Kindern Gartenarbeiten verrichtet oder Spaziergänge unternommen wurden, gespielt, gesungen oder gestrickt und geflickt wurde. Allerdings wurde die Beschäftigung der Kinder trotz dem Ideal der Familienähnlichkeit mit hohen pädagogischen Ansprüchen verbunden. Denn sowohl in Zürich als auch in St. Gallen sollten ausschliesslich ,pädagogisch gebildete“ (Protokoll Frauenverein Zürich, 5.10.1891) Leiterinnen und Leiter ange- 
stellt werden, welche die Kinder in Zürich im pestalozzianischen ${ }^{4}$, in St. Gallen im fröbelschen ${ }^{5}$ Sinne anleiten konnten. Die Auswahl des Personals zeigt also eine grosse Nähe der Horte zur Schule. Der Lehrer Albert Fisler, der als erster Knabenhortleiter in Zürich angestellt wurde, betonte 1898 explizit, dass Charakter und Gesinnung für die Aufgabe als Hortleiterin oder Hortleiter nicht ausreichten, sondern die pädagogische Ausbildung und Erfahrung entscheidend sei: „Wer sich aber je in der Lage gesehen, eine grössere Zahl jugendlicher Quecksilbernaturen im ,Naivetätsstadium‘ unserer Stadtjungen und dazu in ihren Freistunden so zu leiten und zu überwachen, dass sie diese Art Schutzaufsicht nicht als beengenden und drückenden Zwang empfinden, wird bald inne werden, dass ein gewisses Mass von pädagogischer Erfahrung und Übung nicht so leicht entbehrlich ist.“ (Fisler, 1898, S. 20).

In St. Gallen sah es die Kinderhortkommission zwar ebenfalls als Ideal an, ausgebildete Lehrer für die Leitung der Knaben anzustellen, machte aber die Erfahrung, dass es kaum möglich war, berufstätige Lehrer für diese Aufgabe zu finden. Die Kinderhortkommission in St. Gallen entschied daher bereits 1887, ,als Leiter erzieherisch tüchtige Männer aber nicht aktive Lehrer [...] in Aussicht zu nehmen." (Protokoll St. Gallen, 10.1.1887). Dies bedeutete, dass sie in den ersten zwölf Jahren ausgebildete Pfarrer anstellte, was allerdings eine grosse Fluktuation zur Folge hatte, da die Anstellung im Knabenhort von den meisten dieser Männer lediglich als Zwischenverdienst bis zur Wahl in ein Pfarramt genutzt wurde. Schliesslich entschied die Kinderhortkommission St. Gallen, auf der Suche nach einer konstanteren Leitung, Jakob Schmid anzustellen, der weder Pfarrer noch Lehrer, sondern Mesmer ${ }^{6}$ war, der aber ,,durch grossen Takt, Freundlichkeit und Festigkeit in Behandlung der Knaben sich ganz vortrefflich bewährte" (Jahresbericht St. Gallen, 1898, S. 3). Für die Leitung des Mädchenhortes zog es die Kinderhortkommission St. Gallen zwar in Betracht, Frauen, die einen Kurs als Arbeitslehrerin oder Kindergärtnerin absolviert hatten, anzustellen, ausschlaggebend für die Wahl als Mädchenhortleiterin war dann jedoch vielmehr das ,grosse erzieherische Talent“ (Jahresbericht St. Gallen, 1896, S. 8) einer Frau. In St. Gallen waren demnach die pädagogischen Fähigkeiten wichtiger als die Ausbildung der Leiterinnen und Leiter.

4 Heinrich Pestalozzi (1746-1842) wird aufgrund seiner pädagogischen Schriften und des von ihm geführten Erziehungsinstituts in Iferten vielfach als Vorbild für die Armenerziehung herangezogen. Vgl. Oelkers \& Osterwalder, 1995.

5 Friedrich Fröbel (1782-1852) gilt als Begründer der Kindergartenpädagogik. Seine an Pestalozzi angelehnte Pädagogik stellte das kindliche Spiel in den Mittelpunkt. Vgl. Küttel, 1882.

6 Mesmer ist die schweizerische Berufsbezeichnung für Kirchendiener, Sakristan oder Küster, der mit der Aufgabe betraut ist, Hauswartsdienste in einer Kirche zu übernehmen. 
Aus den Berichten der Kinderhortkommission St. Gallen wird deutlich, dass es zudem stets ihre Absicht war, Familien, denen die Erziehung der Kinder aufgrund ihrer Arbeitstätigkeit, wegen Krankheit oder aus anderen Gründen nicht möglich war, zu unterstützen: „Es ist nicht seine [des Kinderhortes] Aufgabe, den Eltern bloss die Besorgung und Ernährung ihrer Kinder erleichtern zu helfen, sondern zu deren Erziehung ihnen behülflich zu sein“ (Jahresbericht St. Gallen, 1895, S. 4). Aus diesem Grund war die Kommission zwar an der Zusammenarbeit mit der Schule interessiert, behielt es sich jedoch vor, möglichst unabhängig davon zu agieren. Im Gegensatz dazu intensivierte die Kinderhortkommission in Zürich die Zusammenarbeit mit den Schulbehörden und den nach der Jahrhundertwende eingesetzten Fürsorgebehörden, etwa wenn es darum ging, ein auffälliges Kind in einer Anstalt zu versorgen (Jahresbericht Zürich, 1910, S. 8). Nach der Jahrhundertwende gab sich die Kommission in Zürich zudem vermehrt die Aufgabe, in den Horten nicht mehr nur die Familienerziehung aufgrund der zeitlichen Abwesenheit der Eltern zu ergänzen, sondern die elterliche Erziehung zu korrigieren (Jahresbericht Zürich, 1915, S. 4f.). Verwahrlosung verstand sie neu als Folge moralischer Fehler der Eltern, woraus sie die Verpflichtung ableitete, in die Erziehungshoheit der Eltern aktiv einzugreifen und deren Fehler zu korrigieren.

\section{Rolle der Sozialen Arbeit}

Die Aufgabe, die Familienerziehung zu kontrollieren und zu korrigieren, welcher sich die Kinderhortkommission Zürich nach der Jahrhundertwende verpflichtet sah, erforderte eine verstärkte Zusammenarbeit mit Fürsorgestellen. Die Zürcher Kommission war daher daran interessiert, die Bestrebungen zu unterstützen, welche erste Ausbildungsangebote für Frauen im Bereich der Sozialen Arbeit schaffen wollten. 1907, noch bevor in Zürich die ersten „Kurse zur Einführung in weibliche Hülfstätigkeit für soziale Aufgaben“ durchgeführt wurden, hiess die Kinderhortkommission Zürich ein Gesuch der Initiantinnen gut, ,dass je 2 bis drei Kursteilnehmerinnen in unseren Mädchenhorten sich an der Leitung beteiligen“ (Protokoll Zürich, 6.7.1907). In den Folgejahren wurden im Mädchen- und in dem späteren Tageshort in Zürich regelmässig sogenannte „Practikantinnen“ (Korrespondenz Gutknecht, 1927) eingesetzt, welche die Kurse für soziale Hilfstätigkeit und ab 1920 die Kurse an der daraus entstandenen sozialen Frauenschule besuchten. Mit Grete Högger übernahm 1925 zudem erstmals eine Absolventin der sozialen Frauenschule die Leitung des Zürcher Mädchenhortes. Dies war kein Zufall, denn die Kurse an dieser neuen Ausbildungsstätte für Soziale Arbeit bereite- 
ten gemäss Kursprogramm unter anderem explizit auf eine Anstellung in einem Kinderhort vor (Ragaz, 1920; Matter, 2011).

Obwohl es in St. Gallen noch keine eigene Ausbildungsstätte für Soziale Arbeit gab, erkannte auch die St. Galler Kinderhortkommission, dass die Horte von diesen neuen Ausbildungsmöglichkeiten profitieren konnten. Dies allerdings weniger zur Verstärkung der interprofessionellen Zusammenarbeit als vielmehr aus dem Grund, dass sich dadurch eine neue Möglichkeit eröffnete, (freiwilliges) Personal für die Hortleitung zu gewinnen. 1909 publizierte die Kommission in ihrem Jahresbericht einen Aufruf zur Suche nach „Töchtern“, die freiwillig im Mädchenhort mithelfen würden und dadurch ,an Geist und Gemüt gefördert, zudem noch in ihrem sozialen Denken und Empfinden weitblickender und weitherziger würden." (Jahresbericht St. Gallen, 1909, S. 4). Die Mitarbeit dieser „Töchter“ bezeichnete die Kinderhortkommission St. Gallen als „,soziale Hilfsarbeit“, womit die Nähe zu den Mädchen- und Frauengruppen für soziale Hilfstätigkeit Alice Salomons deutlich wird, die auch Vorbild der sozialen Frauenschule in Zürich waren. Die bis dahin gemeinnützige, freiwillige Mithilfe von Frauen in den Kinderhorten in St. Gallen wurde somit von der Kinderhortkommission neu zu einem Art Praktikum im Bereich der Sozialen Arbeit für höhere Töchter umgewandelt.

Nach der Jahrhundertwende waren in den Horten in Zürich und St. Gallen nicht nur Frauen mit einer Ausbildung oder Ausbildungsambitionen in der Sozialen Arbeit beschäftigt, sondern es wird aus den Jahresberichten beider Hortkommissionen auch ersichtlich, dass zunehmend sozialpädagogische Anliegen Einzug in die pädagogischen Konzepte fanden. In Zürich zeigte sich dies darin, dass sich besonders die Mädchenhortleiterin Grete Högger gemeinsam mit der Theologin und dem Kommissionsmitglied Rosa Gutknecht für bedürfnisorientierte Angebote einsetzte und erfolgreich einen Tageshort einführte, in welchem die Kinder vor der Schule, über Mittag und nach der Schule betreut werden konnten. Högger machte sich zudem dafür stark, von der Absicht, die Familienerziehung zu korrigieren, abzusehen und den Hort wieder als Unterstützungsangebot für arbeitstätige Eltern zu verstehen. Sie war überzeugt, dass der Hort keinen Ersatz für das Elternhaus bieten könne, aber für die Familien dennoch ,eine grosse Hilfe“ darstelle (Jahresbericht Zürich, 1927, S. 11). In St. Gallen, wo die Horte seit ihrer Gründung als Angebot der Unterstützung verstanden wurden, betonte die Kinderhortkommission nach der Jahrhundertwende, dass neben der Erziehung der Kinder auch das „sozial pädagogische Moment“ einen grossen Stellenwert einnehme, und meinte damit in erster Linie die Verpflegung im Hort, die für viele Kinder ihre Hauptmahlzeit darstellte (Jahresbericht St. Gallen, 1910, S. 7). In beiden Fällen führte der Einbezug der Sozialen Arbeit dazu, dass gegenüber 
einseitig schulischen Ansprüchen dem Unterstützungsauftrag der Horte grösseres Gewicht beigemessen wurde.

Die Horte in der Stadt St. Gallen wurden 1919 dennoch durch die städtischen Schulbehörden übernommen. Die Horte in der Stadt Zürich wurden 1929 zwar durch das Wohlfahrtsamt der Stadt übernommen, zwei Jahre später jedoch ebenfalls dem städtischen Schulamt unterstellt. An beiden Orten werden die Horte als Tagesstrukturen sowohl mit schulischem als auch sozialpädagogischem Personal bis heute weitergeführt.

\section{Fazit}

Der Blick in die Geschichte der Horte für Schulkinder zeigt, dass unter anderem an den Aspekten der Ausbildung und dem Hintergrund des Leitungspersonals sowie den Beschäftigungsformen, die den Kindern angeboten wurden, deutlich wird, inwiefern die Tagesstrukturen als Teil der Schule und/oder als Unterstützung für Familien verstanden und gestaltet wurden. Da sich die Horte im Spannungsfeld zwischen Unterricht und Betreuung und mit dem Aufkommen der Sozialen Arbeit als Profession zudem zwischen Schulpädagogik und Sozialer Arbeit bewegten, wurden diese Aspekte immer wieder unterschiedlich ausgelegt und definiert.

Dieses Spannungsfeld ist bis heute geblieben. Egal unter welcher Trägerschaft Tagesstrukturen geführt werden, sie stehen immer im Spannungsfeld zwischen Unterricht und Betreuung sowie zwischen Schulpädagogik und Sozialer Arbeit. Die Frage danach, wie viel Schulnähe oder wie viel sozialpädagogische Anliegen das pädagogische Konzept beinhalten soll oder ob Lehrpersonen, Personen mit einer Ausbildung in Sozialer Arbeit oder ohne pädagogische Ausbildung dem Auftrag der Tagesstrukturen am besten gerecht werden können, bleibt nach wie vor ungeklärt (vgl. EDK, 2011, S. 27f.). Die Geschichte der ersten Horte in der Deutschschweiz zeigt, dass je nach Hintergrund des Personals eine grössere Nähe entweder zur Schule oder zur Sozialen Arbeit bzw. zur Fürsorge angestrebt wird. Eine interprofessionelle Zusammenarbeit ermöglicht(e) zwar vielfältigere Kooperationsformen, kann jedoch auch zu einer Vormachtstellung gegenüber den Eltern führen und etwa zur stärkeren Einflussnahme oder gar Kontrolle der Familien verleiten. Sollen Tagesstrukturen nicht nur Teil der Schulpädagogik sein, sondern auch das Professionswissen der Sozialen Arbeit einbezogen werden, scheint das Bewusstsein für dieses Gefahrenpotential, aber vor allem auch eine reflexive, dialogische Haltung zwischen den Professionen einerseits und den Familien als Nutzern der Tagesstrukturen andererseits von grosser Wichtigkeit. Die Diskussion um Tagesstrukturen wird wie schon in der Vergangenheit auch in 
Zukunft politischen und pädagogischen Konjunkturen unterworfen sein. Erst durch die interprofessionelle Zusammenarbeit von Fachpersonen der Schulpädagogik und der Sozialen Arbeit können diese reflektiert und relativiert werden. Und erst durch die Zusammenarbeit kann auch ermöglicht werden, dass in den Tagesstrukturen sowohl schulische als auch sozialpädagogische Anliegen vereint werden.

\section{Quellen}

Sozarch Ar 495.10:1: Protokolle des Gemeinnützigen Frauenvereins Sektion Zürich, 1889-1928.

StadtASG 4/1/58: Jahresberichte Kinderhortkommission St. Gallen, 1887-1917.

StadtASG 4/1/58: Protokollbücher Kinderhortkommission St. Gallen, 1887-1912.

Stadtarchiv Zürich VII.53: Jahresberichte Jugendhortkommission Zürich I, 18871926.

Stadtarchiv Zürich VII.53: Protokolle Kinderhortkommission Zürich, 1895-1909.

Stadtarchiv Zürich VII.53: Korrespondenz Rosa Gutknecht, 1927.

\section{Sekundärliteratur}

BFS (2015): Typologie der Betreuungsformen. Verfügbar unter: http://www.netzwerk-kinderbetreuung.ch/media/filer_public/aa/7c/aa7c9235c0ae-4639-8703-95c7e5e5b349/bfs_feb_statistik_typologie_20150709.pdf [Zugriff: 27.02.2018].

EDK (2011): Interkantonale Vereinbarung über die Harmonisierung der obligatorischen Schule (HarmoS-Konkordat) vom 14. Juni 2007. Verfügbar unter: https://edudoc.ch/record/96777/files/harmos-konkordatd.pdf [Zugriff: 27.02.2018].

Fisler, A. (1898): Städtische Jugend und Jugendhorte. Achtundneunzigstes Neujahrsblatt der Zürcherischen Hülfsgesellschaft, 1898.

Grunder, H.-U. (2008): Ganztagsschulen in der Schweiz - Anstösse aus der Schulgeschichte. In: S. Larcher Klee \& B. Grubenmann (Hrsg.), Tagesstrukturen als sozial- und bildungspolitische Herausforderung. Bern 2008, S. 101-116.

Küttel, C. (1882): Der Fröbel'sche Kindergarten in der Schweiz. Zürich 1882.

Matter, S. (2011): Der Armut auf den Leib rücken. Die Professionalisierung der Sozialen Arbeit in der Schweiz (1900-1960). Zürich 2011.

Oelkers, J., Osterwalder, F. (1995): Pestalozzi - Umfeld und Rezeption. Studien zur Historisierung einer Legende. Basel 1995.

Ragaz, C. (1920): Soziale Arbeitsschule Zürich. Neue Wege 14/10, 1920, S. 437-439. Rolle, J., Kessberg, E. (1988): Der Hort im Spiegel seiner Geschichte. Stuttgart 1988.

Schüpbach, M., Frei, L., Nieuwenboom, W. (2017): Tagesschulen. Ein Überblick. Wiesbaden 2017. 


\section{Zur Qualität ganztägiger Bildung}

Nina Thieme

Spätestens seit der Veröffentlichung der ersten PISA-Ergebnisse (vgl. Deutsches PISA-Konsortium 2001), durch die ein unterdurchschnittlich schlechtes Abschneiden deutscher Schülerinnen und Schüler mit Blick auf die untersuchten Bereiche der Lesekompetenz, der mathematischen und der naturwissenschaftlichen Grundbildung attestiert worden ist, steht in Deutschland das „Thema ,Ganztagsschule“ ganz oben auf der Agenda der aktuellen Bildungsreformdebatte" (Wissenschaftlicher Beirat für Familienfragen beim BMFSFJ 2006, S. 11). Durch das vom Bund getragene Investitionsprogramm „Zukunft Bildung und Betreuung" (IZBB) (vgl. u.a. BMBF 2009) ist ein umfassender Ausbau von Ganztagsschulen initiiert worden, verbunden mit dem Ziel einer Qualitätsverbesserung des deutschen Bildungssystems durch qualitativ hochwertige ganztägige Bildung. In diesem Zusammenhang wird das, was die Qualität ganztägiger Bildung ausmacht, eher aus einer objektivistischen Perspektive bestimmt (vgl. Kapitel 1) bei gleichzeitiger Nichtberücksichtigung eines relationalen Qualitätsverständnisses, gemäß dem Qualität in und durch Aushandlungsprozesse/n bestimmt wird (vgl. Kapitel 2). Inwieweit Ganztagsschulen einen Raum darstellen, in dem solche Aushandlungen über die Qualität ganztägiger Bildung erfolgen können (vgl. Kapitel 4), wird anschließend an die Darlegung der Aushandlungsmöglichkeiten der an ganztägiger Bildung im Wesentlichen beteiligten Institutionen der Schule und der Kinder- und Jugendarbeit (vgl. Kapitel 3) reflektiert.

\section{Einleitende Bemerkungen: zur bildungspolitischen Forderung von Qualität ganztägiger Bildung}

Mittlerweile sind Ganztagsschulen in der deutschen Bildungslandschaft fest verankert. Durch das vom Bund von 2003 bis 2009 getragene Investitions- 
programm „Zukunft Bildung und Betreuung“ (IZBB) (vgl. u.a. BMBF 2009) ist ein umfassender Ausbau von Ganztagsschulen initiiert worden, die sich gemäß der Kultusministerkonferenz (KMK) dadurch kennzeichnen, dass

- ,an mindestens drei Tagen in der Woche ein ganztägiges Angebot für die Schülerinnen und Schüler bereitgestellt wird, das täglich mindestens sieben Zeitstunden umfasst,

- an allen Tagen des Ganztagsschulbetriebs den teilnehmenden Schülerinnen und Schülern ein Mittagessen bereitgestellt wird,

- die Ganztagsangebote unter der Aufsicht und Verantwortung der Schulleitung organisiert und in enger Kooperation mit der Schulleitung durchgeführt werden sowie in einem konzeptionellen Zusammenhang mit dem Unterricht stehen“" (KMK 2013, S. 4).

Eines der wesentlichen Ziele dieses mit vier Milliarden ausgestatteten Investitionsprogramms bestand in einer nachhaltigen „Qualitätsverbesserung unseres Bildungssystems“ (BMBF 2003a). Die anvisierte Qualitätsverbesserung sollte dazu beitragen, so formulierte es die von 1998 bis 2005 amtierende Bundesministerin für Bildung und Forschung, Edelgard Bulmahn, in einer Imagebroschüre des Bundesministeriums für Bildung und Forschung (BMBF) zum Investitionsprogramm, „das deutsche Bildungssystem in zehn Jahren wieder an die Weltspitze [zu] bringen. Denn von der Qualität der Bildung hängt unsere Zukunft ab“ (2003b). Diese nicht nur retrospektiv ambitioniert anmutende und auch präzisierungsbedürftige (Heraus-)Forderung, das deutsche Bildungssystem innerhalb einer Dekade ,wieder an die Weltspitze [zu] bringen“ (ebd.), solle in ganztägigen Settings durch

- eine ,individuelle Förderung und [ein] Eröffnen von Lernchancen“ (ebd., S. 7),

- $\quad$ eine „Veränderung von Unterricht und Lernkultur“ (ebd.),

- „Soziales Lernen“( (ebd.),

- „Partizipation“ (ebd.),

- eine „Öffnung von Schule“ (ebd.),

- „Kreative Freizeitgestaltung“ (ebd.) und

- eine „Qualifizierung des Personals“ (ebd.) realisiert werden.

Durch die erfolgte Festlegung solcher Merkmale, die zugleich eine qualitativ hochwertige ganztägige Bildung ausmachen würden, wird die Annahme vermittelt, dass Qualität ,in Bezug auf inhaltliche Aspekte des Gegenstandsbereichs zu bestimmen sei“ (Oelerich 2005, S. 5). Qualität wird also, in einer solchen als objektivistisch zu bezeichnenden Perspektive, durch die Beschaffenheit des Gegenstands selbst zu begründen versucht (vgl. dazu auch Neumann \& Honig 2009, S. 196; Schaarschuch \& Schnurr 2004, S. 321). Damit 
einhergehend wird die Möglichkeit einer von Akteurinnen und Akteuren unabhängig erfolgenden Festlegung von Qualität markiert; unberücksichtigt bleibt, dass Qualität ,relativ zu demjenigen [ist], der diesen Begriff verwendet sowie abhängig [ist] von Kontexten, in denen er verwendet wird" (Harvey \& Green 2000, S. 17).

An diesem Punkt setzen theoretische Positionen an, die ein objektivistisches Qualitätsverständnis „,ür unangemessen halten und nicht die gegenstandsbezogenen Inhalte von Angeboten, sondern vielmehr die Aushandlungsprozesse zwischen den beteiligten Akteuren um die Qualität eines Angebots in den Mittelpunkt stellen“ (Oelerich 2005, S. 5).

\title{
2. Qualität als relationales, aushandlungsbedürftiges Konstrukt
}

\author{
„Also, wo ich so denke, selbst wenn die \\ Angebote wirklich qualitativ hochwertig sind, dann heißt es \\ zum Teil aber nicht unbedingt, dass es dann eben entsprechend \\ in der Wahrnehmung des Schülers so ist."
}

(Hartmut Lietzau, Lehrer an einem gebundenen Ganztagsgymnasium)

Auch wenn in diesem Zitat, das aus einem narrativen, im Rahmen der BiRBi-Pro-Studie $^{1}$ geführten Interview stammt, einleitend ein ,(normative[r]) Essentialismus“ (Neumann \& Honig 2009, S. 196) deutlich wird, legt es gleichzeitig nahe, dass Qualität nur im gemeinsamen Dialog in und durch Aushandlungsprozesse/n bestimmt werden kann. Einem solchen Verständnis von Qualität als relationalem Konstrukt (vgl. u.a. Schaarschuch \& Schnurr 2004) entsprechend, müssten die an ganztägiger Bildung beteiligten Akteurinnen und Akteure - vornehmlich Lehrkräfte, Professionelle der Sozialen Arbeit und Schülerinnen und Schüler - ins Zentrum gerückt sowie deren „Interessenlagen [und damit verbundene Perspektiven] [...] [sowie] die Verhältnisse und Beziehungen, die sie miteinander verbinden, systematisch berück-

1 Mit der BiRBi-Pro-Studie („Bildungsgerechtigkeit oder Reproduktion von Bildungsungerechtigkeit durch schul- und sozialpädagogische Professionelle. Fallrekonstruktionen am Beispiel ganztägiger Arrangements") sind wir in einer professionenvergleichenden Perspektive folgenden Fragestellungen nachgegangen: 1. Wie deuten Professionelle der Sozialen Arbeit und Lehrkräfte in Ganztagsschulen das an sie adressierte Leitziel einer Reduktion von Bildungsungerechtigkeit? 2. Wie (re-)produzieren sie, vor dem Hintergrund der von ihnen artikulierten Sichtweisen, Bildungsungerechtigkeit? Alle Daten, die im Rahmen dieser von Oktober 2011 bis Januar 2015 vom Bundesministerium für Bildung und Forschung (BMBF) geförderten Studie (vgl. u.a. Thieme, Faller \& Heinrich 2012) erhoben worden sind, sind anonymisiert worden. 
sichtigt [werden]“ (ebd., S. 310). Gleichzeitig, und dies wird auch durch das Zitat deutlich, ist davon auszugehen, dass die an ganztägiger Bildung beteiligten Akteurinnen und Akteure ,auf Grund ihrer Positionierung in diesem Feld unterschiedliche und (partiell) widersprüchliche Interessen [aufweisen]“ (ebd., S. 312; Hervorheb. im Original), wodurch auch erst das Erfordernis resultiert, Qualität auszuhandeln.

Was kann unter Aushandlung verstanden werden?

Als gängiger Terminus in der deutschen Sprache lässt sich Aushandlung analytisch betrachtet - folgendermaßen fassen: Ausgangspunkt sind (zumindest partiell) divergierende Interessenlagen von zwei oder mehr im Prinzip gleichberechtigten Personen bzw. Gruppen, die in einem gemeinsamen Kommunikationsprozess die eigenen Interessen zu artikulieren vermögen, diese in Bezug zu den geäußerten Vorstellungen des Gegenübers setzen und daran anschließend Vorschläge finden, ,that meet both sides' demands or needs to a mutually satisfactory extent" (Zartmann 2002, S. 7; vgl. auch Strauss 1978).

Solche Aushandlungen seien eingebettet in ,,negotiation context $[\mathrm{s}]^{6.2}$ (Strauss 1978, S. 237; Hervorheb. im Original), in Kontexte also, die durch bestimmte Merkmale gekennzeichnet sind, beispielsweise durch die Anzahl der an der Aushandlung beteiligten Akteurinnen und Akteure, durch die Anzahl und Komplexität der auszuhandelnden Sachverhalte sowie durch die Möglichkeit, Aushandlung zu beenden bzw. zu vermeiden. Im Vergleich zu diesen, der Spezifizierung von Aushandlungskontexten dienenden Merkmalen führt Strauss ein Merkmal an, das für Aushandlungskontexte konstitutiv ist, durch das Aushandlung also erst grundlegend möglich wird: ,The relative balance of power exhibited by the respective parties in the negotiation itself" (ebd.; Hervorheb. im Original).

\section{Zur Aushandlungsmöglichkeit in Schule und Kinder- und Jugendarbeit}

Geht man von der Annahme aus, dass Qualität ein relationales, der Aushandlung bedürfendes Konstrukt sei (vgl. u.a. Schaarschuch \& Schnurr 2004), ist mit Blick auf den ganztagsschulischen Kontext die Frage zu stellen, inwieweit Ganztagsschule einen ,negotiation context" (Strauss 1978, S. 237; Hervorheb. im Original) darstellt, in dem alle an ganztägiger Bildung beteiligten Akteurinnen und Akteure, vor allem Lehrkräfte, Professionelle der Sozialen

2 „The term negotiation context refers specifically to the structural properties entering very directly as conditions into the cours of the negotiation itself" (Strauss 1978, S. 237f.; Hervorheb. im Original). 
Arbeit und Schülerinnen sowie Schüler, ihre Interessenlagen artikulieren und damit verbundene Perspektiven auf die (auszuhandelnde) Qualität dieser Angebote annähernd gleichberechtigt (vgl. ebd.) einbringen können, wodurch eine Aushandlung, konstitutionstheoretisch gesprochen, erst möglich werden würde.

Hinsichtlich der Institution Schule könnte ein solches, für Aushandlungsprozesse notwendiges, im Prinzip gleichberechtigtes Einbringen eigener Perspektiven sowie ein gleichberechtigtes Vertreten eigener Interessenlagen erschwert sein, da durch die Aufgaben der Institution Schule vor allem mit Blick auf Interaktionen zwischen Schülerinnen sowie Schülern und Lehrkräften „ein spezifisches Grundgerüst fest[gelegt]“ (Ulich 2001, S. 77) werde:

- „In der Schule sollen zunächst einmal Kenntnisse und Fähigkeiten vermittelt und damit die nachwachsende Generation qualifiziert werden. Allein dadurch entsteht schon ein unvermeidliches Moment von Ungleichheit; denn die Lehrer/innen verfügen über jenes Wissen, das die Schüler/innen noch nicht besitzen. Im Qualifikationsvorsprung der Lehrer/innen gegenüber Schüler/innen liegt deshalb eine wichtige Quelle ihrer Macht.

- Die Schule soll die Jugendlichen im Hinblick auf unterschiedliche Schulabschlüsse und auf den Zugang zu beruflichen Positionen auslösen; diese Aufgabe erfordert individuelle Leistungsbewertungen und Notenzuteilungen, was wiederum die Abhängigkeit der Schüler/innen von Lehrer/innen verstärkt.

- Drittens soll Schule auf gesellschaftlich anerkannte, allerdings zunehmend umstrittene bzw. unklare Wertvorstellungen hin erziehen und damit zur sozialen Integration der Schüler/innen beitragen. Lehrer/innen kontrollieren z.B. die Einhaltung von Grundregeln des sozialen Umgangs in der Schule und können Regelverletzungen der Schüler/innen sanktionieren. Auch dies ist ein zentraler Machtaspekt der Lehrer/innen“ (ebd., S. 78; Hervorheb. im Original).

Anders gestaltet sich die Situation mit Blick auf das sozialpädagogische, von freien wie öffentlichen Trägern gestaltete Handlungsfeld der Kinder- und Jugendarbeit, durch deren Angebote Ganztagsschule überhaupt erst ganztägig werde (vgl. Coelen \& Wahner-Liesecke 2009, S. 241). Organisationsstrukturell bedingt ist Kinder- und Jugendarbeit, vor allem die offene Kinder- und Jugendarbeit, eher aushandlungsorientiert, ,weil die Sicherheit bietenden Institutionalisierungen und Routinisierungen der Handlungsabläufe" (Cloos et al. 2009, S. 194), im Gegensatz zu anderen professionellen Handlungsfeldern wie beispielsweise der Schule, so nicht gegeben seien, da „,nicht präzise festgelegt ist, was [...] eigentlich geschehen soll“" (Cloos \& Köngeter 2009, S. 88). 


\section{Abschließende Reflexionen: Ganztagsschule als Raum der Aushandlung von Qualität ganztägiger Bildung? Zur (möglichen) Bedeutsamkeit der Kinder- und Jugendarbeit}

In bildungspolitischen Forderungen von Qualität ganztägiger Bildung materialisiert sich ein objektivistisches Qualitätsverständnis, gemäß dem versucht wird, Qualität ,,in Bezug auf inhaltliche Aspekte des Gegenstandsbereichs zu bestimmen“ (Oelerich 2005, S. 5). Demgegenüber werden mit einem relationalen Qualitätsverständnis ,vielmehr die Aushandlungsprozesse zwischen den beteiligten Akteuren um die Qualität eines Angebots in den Mittelpunkt“ (ebd.) gestellt.

Ein solches Qualitätsverständnis zugrunde legend, stellt sich mit Blick auf ganztägige Bildung (zunächst) weniger die Frage, was als Qualität gilt, sondern ob Ganztagsschule, auch durch die Beteiligung einer strukturell bedingt eher aushandlungsorientierten Kinder- und Jugendarbeit, zu einem Raum wird, in dem Akteurinnen und Akteure, vor allem Lehrkräfte, Professionelle der Sozialen Arbeit und Schülerinnen und Schüler, ihre Interessenlagen artikulieren und damit verbundene Perspektiven auf die (auszuhandelnde) Qualität ganztägiger Bildung im Prinzip gleichberechtigt einbringen können.

Die Beantwortung dieser Frage hängt von verschiedenen, auf unterschiedlichen Ebenen angesiedelten und zusammenhängenden Faktoren ab:

Auf der organisationalen Ebene ist vor allem von Bedeutung, inwieweit durch die Einführung des Ganztags eine strukturelle Veränderung von Schule erfolgt. Wenn primär „nur mehr vom Gleichen geboten [wird]“ (Oelkers 2010, S. 46; Hervorheb. im Original), erscheint es vor dem Hintergrund der vornehmlich strukturell bedingten "sehr ungleichen Beziehung“ (Uhlich 2001, S. 77; Hervorheb. im Original) zwischen Schülerinnen sowie Schülern und Lehrkräften eher unplausibel, dass Ganztagsschule zu einem Raum der Aushandlung von Qualität ganztägiger Bildung wird. Erfolgt demgegenüber eine Öffnung von Schule derart, dass Bildungsangebote außerschulischer Partner, vor allem der Kinder- und Jugendarbeit, einbezogen werden, dürfte die Gestaltung der Verbindung von Unterricht und außerunterrichtlichen Angeboten eine Rolle spielen: Im Rahmen eines additiven Modells, in dem Unterricht und außerunterrichtliche Angebote ,eher unverbunden nebeneinander [ge]stellt" (Oelerich 2007, S. 18; Hervorheb. im Original) sind, werden Aushandlungsmöglichkeiten von Qualität potentiell eher auf Seiten der Kinder- und Jugendarbeit verbleiben. Wenn, einem integrativen Modell entsprechend, dagegen ,eine systematische Integration von unterrichtlichen und nicht-unterrichtlichen Anteilen" (ebd.; Hervorheb. im Original) erfolgt, wäre 
zunächst in den Blick zu nehmen, wie sich die multiprofessionelle Zusammenarbeit von Lehrkräften und Professionellen der Sozialen Arbeit faktisch gestaltet.

Bezüglich dieser Ebene lassen sich im Diskurs über multiprofessionelle Kooperation in pädagogischen Handlungskontexten zwei Argumentationslinien identifizieren (vgl. u.a. Kunze 2018; Silkenbeumer, Thieme \& Kunze 2017): Einem „Integrationsansatz“ (Kunze 2018) folgend, werde davon ausgegangen, dass sich multiprofessionelle Kooperation durch eine „Kopplung und Verschmelzung der unterschiedlichen Expertisen und Reflexionsmuster der Beteiligten“ (ebd.) kennzeichne. Demnach könnte eine multiprofessionelle Kooperation mit Blick auf die Profession der Lehrkräfte für diese das Potential eröffnen, aushandlungsorientierter zu werden. Geht man, gegenläufig dazu, von einem „Differenzierungsansatz“ (ebd.) aus, ist anzunehmen, dass durch multiprofessionelle Kooperationen fachliche und aufgabenbezogene Differenzen gerade nicht aufgelöst, sondern aufrechterhalten werden. In dem Fall erscheint es plausibel, dass eine Aushandlungsorientierung auf Seiten der Kinder- und Jugendarbeit verbleiben dürfte.

Grundsätzlich legen die Ausführungen nahe, dass unter bestimmten Voraussetzungen eine strukturell bedingt eher aushandlungsorientierte Kinderund Jugendarbeit einen bedeutsamen Beitrag dazu leisten könnte, Ganztagsschule zu einem Raum der Aushandlung von Qualität ganztägiger Bildung werden zu lassen. Die konkrete Ausgestaltung eines solchen Beitrags zu klären - unter Berücksichtigung der angeführten Faktoren -, bleibt jedoch letztlich eine empirische Aufgabe.

\section{Literatur}

BMBF (2003a): Verwaltungsvereinbarung Investitionsprogramm „Zukunft Bildung und Betreuung“ 2003 - 2007. URL:

http://www.ganztagsschulen.org/_media/20030512_verwaltungsvereinbarung_zu kunft_bildung_und_betreuung.pdf; Datum des Zugriffs: 29.10.2017.

BMBF (2003b): Investitionsprogramm „Zukunft Bildung und Betreuung“. Ganztagsschulen. Zeit für mehr. URL:

http://ganztag.nepomucenum.de/offen/praesentation/imagbroschuere_bmbf.pdf;

Datum des Zugriffs: 29.10.2017.

BMBF (2009): Gut angelegt. Das Investitionsprogramm Zukunft Bildung und Betreuung. Berlin.

Coelen, Thomas \& Wahner-Liesecke, Ingrid (2009): Jugendarbeit kann auch mit (Ganztags-)Schulen wirken. In: Lindner, Werner [Hrsg.]: Kinder- und Jugendarbeit wirkt. Aktuelle und ausgewählte Evaluationsergebnisse der Kinder- und Jugendarbeit, 2. Auflage. Wiesbaden: VS, S. 241-260. 
Cloos, Peter \& Köngeter, Stefan (2009): ,uns war ma langweilig, da ham wir das JUZ entdeckt" - Empirische Befunde zum Zugang von Jugendlichen zur Jugendarbeit. In: Lindner, Werner [Hrsg.]: Kinder- und Jugendarbeit wirkt. Aktuelle und ausgewählte Evaluationsergebnisse der Kinder- und Jugendarbeit, 2. Auflage. Wiesbaden: VS, S. 81-94.

Cloos, Peter; Köngeter, Stefan; Müller, Burkhard \& Thole, Werner (2009): Die Pädagogik der Kinder- und Jugendarbeit, 2., durchgesehene Auflage. Wiesbaden: VS.

Deutsches PISA-Konsortium [Hrsg.] (2001): PISA 2000: Basiskompetenzen von Schülerinnen und Schülern im internationalen Vergleich. Opladen: Leske + Budrich.

Harvey, Lee \& Green, Diana (2000): Qualität definieren. Fünf unterschiedliche Ansätze. In: Zeitschrift für Pädagogik 46, 41. Beiheft, S. 17-39.

KMK (2013): Allgemein bildende Schulen in Ganztagsform in den Ländern in der Bundesrepublik Deutschland - Statistik 2007 bis 2011 -. URL: http://www.kmk.org/fileadmin/pdf/Statistik/GTS_2011_Bericht.pdf; Datum des Zugriffs: 29.10.2017.

Kunze, Katharina (2018): „Niemandem die Scheibe Brot vom Teller ziehen.“ Zuständigkeitsunsicherheit als Herausforderung multiprofessioneller Kooperationsbeziehungen. Erscheint in: Boller, Sebastian et al. [Hrsg.]: Friedrich Jahresheft.

Neumann, Sascha \& Honig, Michael-Sebastian (2009): Das Maß der Dinge. Qualitätsforschung im pädagogischen Feld. In: Friebertshäuser, Barbara; Rieger-Ladich, Markus \& Wigger, Lothar [Hrsg.]: Reflexive Erziehungswissenschaft. Forschungsperspektiven im Anschluss an Pierre Bourdieu, 2., durchgesehene und erweiterte Auflage. Wiesbaden: VS, S. 190-210.

Oelerich, Gertrud (2005): Bestandsaufnahme und Auswertung der aktuellen Literatur und Forschungslage zu Ganztagsschulkonzepten und deren Qualitätsmerkmalen in Deutschland. Expertise zum BLK-Modellversuch ,Lernen für den GanzTag ${ }^{6}-$ Entwicklung von Qualifikationsprofilen und Fortbildungsbausteinen für pädagogisches Personal an Ganztagsschulen. URL:

http://www.ganztag-blk.de/cms/upload/pdf/blk/Oelerich_Qualittsmerkmale.pdf; Datum des Zugriffs: 02.11.2017.

Oelerich, Gertrud (2007): Ganztagsschulen und Ganztagsangebote in Deutschland Schwerpunkte, Entwicklungen und Diskurse. In: Bettmer, Franz et al. [Hrsg.]: Ganztagsschule als Forschungsfeld. Theoretische Klärungen, Forschungsdesigns und Konsequenzen für die Praxisentwicklung. Wiesbaden: VS, S. 13-42.

Oelkers, Jürgen (2010): Chancengleichheit, Ganztagsschule und Qualitätssicherung. In: Nerowski, Christian \& Weier, Ursula [Hrsg.]: Ganztagsschule organisieren ganztags Unterricht gestalten. Bamberg: University of Bamberg Press, S. 33-57.

Schaarschuch, Andreas \& Schnurr, Stefan (2004): Konflikte um Qualität. Konturen eines relationalen Qualitätsbegriffs. In: Beckmann, Christof; Otto, Hans-Uwe; Richter, Martina \& Schrödter, Mark [Hrsg.]: Qualität in der Sozialen Arbeit. Zwischen Nutzerinteresse und Kostenkontrolle. Wiesbaden: VS, S. 309-329.

Silkenbeumer, Mirja; Thieme, Nina \& Kunze, Katharina (2017): Kooperation in multiprofessionellen Handlungskontexten. Zur Frage beruflicher Zuständigkeit/en Sozialer Arbeit. In: Thieme, Nina \& Silkenbeumer, Mirja [Hrsg.]: Die herausge- 
forderte Profession. Soziale Arbeit in multiprofessionellen Handlungskontexten. 14. Sonderheft der Zeitschrift , neue praxis'.

Strauss, Anselm (1978): Negotiations. Varieties, Contexts, Processes, and Social Order. San Francisco, Washington \& London: Jossey-Bass Publishers.

Thieme, Nina; Faller, Christiane \& Heinrich, Martin (2012): Bildungsgerechtigkeit oder Reproduktion von Bildungsungerechtigkeit durch schul- und sozialpädagogische Professionelle - BiRBi-Pro. In: Soziale Passagen 4, Heft 1, S. 159-162.

Ulich, Klaus (2001): Einführung in die Sozialpsychologie der Schule. Weinheim \& Basel: Beltz.

Wissenschaftlicher Beirat für Familienfragen beim BMFSFJ (2006): Ganztagsschule - eine Chance für Familien.

http://www.bmfsfj.de/BMFSFJ/Service/publikationen,did=93306.html; Datum des Zugriffs: 06.12.2017.

Zartmann, William (2002): What I Want to Know about Negotiations. In: International Negotiation 7, Heft 1, S. 5-15. 


\title{
Wie Schulsozialarbeit und Schule kooperieren: Fünf Merkmale interdisziplinärer Zusammenarbeit zwischen Schulsozialarbeitenden und Lehrpersonen
}

\author{
Monique Brunner, Roger Pfiffner, Simone Ambord, \\ Ueli Hostettler
}

\section{Einleitung}

Im Schulalltag werden Lehrpersonen häufig mit Schwierigkeiten von Schülerinnen und Schülern konfrontiert, die den Schulbetrieb beeinträchtigen können: Verhaltensprobleme, Leistungsschwäche aufgrund familiärer Probleme, Kindeswohlgefährdungen, Gewalttätigkeiten im Umfeld der Schule sowie Sucht- oder Integrationsprobleme. Die angemessene Bearbeitung solcher Problemstellungen kann die Schulen und Lehrkräfte schnell überfordern, was seit Ende der 1990er Jahre zur raschen Verbreitung der Schulsozialarbeit an deutschsprachigen Schulen geführt hat (Hafen, 2005).

Die Schulsozialarbeit ist ein eigenständiges Handlungsfeld der Kinderund Jugendhilfe, richtet sich aber an die gleiche Adressatengruppe wie die Schule. Mit der Verbesserung der Entwicklungsbedingungen von Kindern und Jugendlichen verfolgt sie zudem dieselbe übergeordnete Zielsetzung wie die Lehrpersonen (Olk, Bathke \& Hartnuss, 2000). Dadurch entstehen Schnittstellen und Aufgabenüberschneidungen in den Bereichen Prävention, Früherkennung und Frühintervention sowie in der Elternarbeit. Dies setzt hohe Anforderungen an die Arbeitsteilung im schulischen Alltag und an die $\mathrm{Zu}-$ sammenarbeit verschiedener Akteurinnen und Akteure.

Ein Grossteil der Forschungsliteratur zur Kooperation im Bildungs- und Sozialwesen geht davon aus, dass eine gelingende Zusammenarbeit zwischen verschiedenen Fachbereichen zum Vorteil der Zielgruppen sowie der beteiligten Fachpersonen erfolgt (Mellin, Anderson-Butcher \& Bronstein, 2011; Stratz \& Wiklund, 2013). Gleichzeitig werden zahlreiche Herausforderungen für gelingende Zusammenarbeitsformen identifiziert, was erklärt, dass die Zusammenarbeit zwischen Schule und Schulsozialarbeit nicht immer unproblematisch ist (Olk, 2005). Diese Erkenntnis stammt primär aus Evaluationsstudien, die sich auf einzelne Schulsozialarbeitsangebote in kleineren Einzugsgebieten beziehen. Eine verallgemeinerbare empirische Grundlage zu den Kooperationsformen zwischen Schulsozialarbeit und Schule lag für die Schweiz bisher nicht vor. 
Im Gegensatz zur bisherigen Evaluationsforschung weist die hier präsentierte Studie eine umfassendere Datengrundlage auf und arbeitet mit einer theoretisch fundierten Definition von interdisziplinärer Zusammenarbeit. Die Untersuchung geht der Frage nach, welche Merkmale die interdisziplinäre Zusammenarbeit zwischen Schulsozialarbeit und Schule auszeichnen. Dafür wurden 173 Schulsozialarbeitende in vier Kantonen online dazu befragt, wie sie die gegenseitige Kooperation mit den Lehrpersonen einschätzen. Das der Untersuchung zugrundeliegende Kooperationsverständnis basiert auf dem «Modell für interdisziplinäre Zusammenarbeit» (Bronstein, 2003), das anschliessend beschrieben wird. Danach folgen eine Erläuterung der Datenbasis und des methodischen Vorgehens sowie die Präsentation der Ergebnisse. Abschliessend geht der Beitrag auf die Implikationen unserer Analyse für die Zusammenarbeit von Schulsozialarbeit und Schule ein.

\section{Interdisziplinäre Zusammenarbeit an Schulen: Bedeutung und Herausforderungen}

In der Literatur finden sich unterschiedliche Definitionen und Operationalisierungen berufsübergreifender Zusammenarbeitsformen. Das «Modell der interdisziplinären Zusammenarbeit» von Bronstein (2003) ist ein bekanntes Modell aus der Sozialen Arbeit, das in vergleichbaren empirischen Studien im englischsprachigen Raum bereits angewendet wurde. Das Modell definiert interdisziplinäre Zusammenarbeit als einen «effektiven zwischenmenschlichen Prozess, der das Erreichen von Zielen erlaubt, die von individuellen Angehörigen einer Profession allein nicht erreicht werden können» (Bronstein, 2003, S. 299; Übersetzung der Autorinnen und Autoren). Zur Entwicklung des Modells hat Bronstein verschiedene Ansätze interdisziplinärer $\mathrm{Zu}$ sammenarbeit analysiert und die sie kennzeichnenden, gemeinsamen Aspekte herausgearbeitet. Daraus resultierten fünf zusammenhängende Komponenten einer erfolgreichen Zusammenarbeit: Interdependenz, gemeinsame Zielvorstellungen, Flexibilität, neue gemeinsame professionelle Aktivitäten und Reflexion des Arbeitsprozesses (Abbildung 1). 


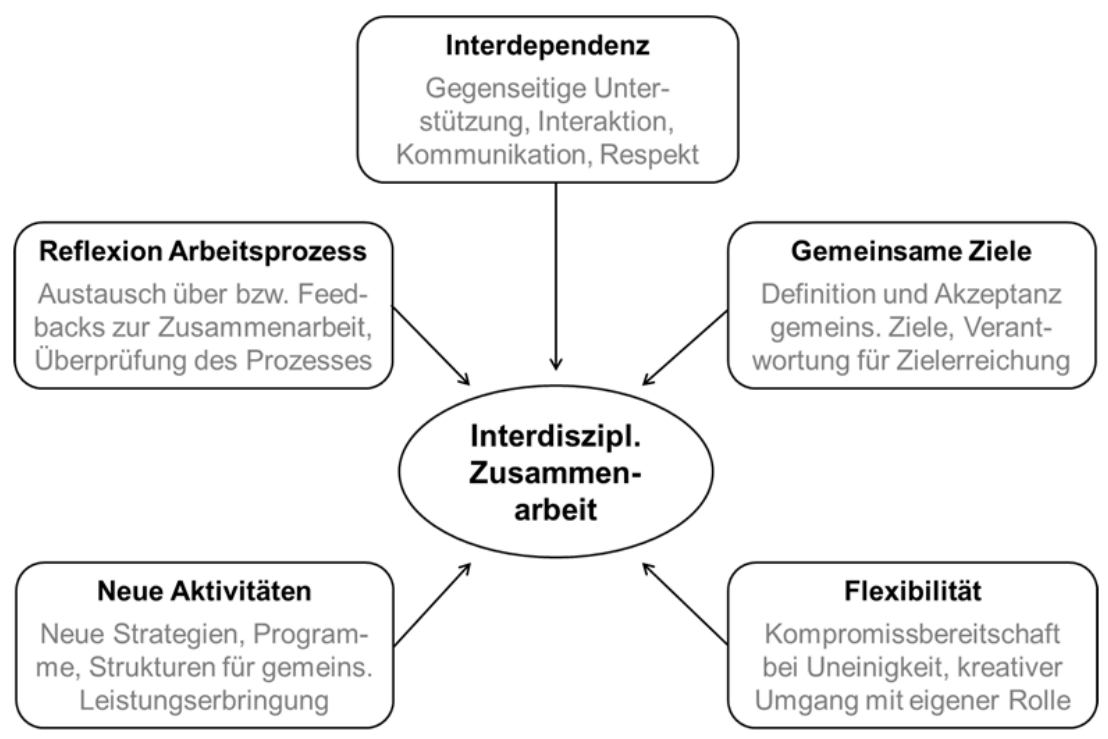

Abbildung 1: Fünf Komponenten der interdisziplinären Zusammenarbeit

Interdisziplinäre Kooperationsformen werden in der Forschungsliteratur als notwendig erachtet für die frühzeitige Erkennung und Bearbeitung von komplexen zusammenhängenden Schwierigkeiten von Kindern, Jugendlichen und Familien (Higham \& Yeomans, 2010; Darlington, Healy \& Feeney, 2010). Eine intensive Zusammenarbeit zwischen Schulsozialarbeit und Schule trägt nach Baier und Heeg (2011, S. 133) dazu bei, dass Gefährdungen von Schülerinnen und Schülern früher erkannt und bearbeitet werden können. Die Öffnung der Schulen gegenüber Angeboten der Kinder- und Jugendhilfe, eine bessere Berücksichtigung der lebensweltlichen Bedürfnisse von Kindern und Jugendlichen sowie die Förderung der Schulentwicklung können weitere positive Auswirkungen sein (Olk \& Speck, 2012). Wie Evaluationsstudien gezeigt haben, wird allerdings die Zusammenarbeit zwischen verschiedenen Berufsgruppen nicht in allen Schulen gleichermassen gepflegt (z.B. Pfiffner \& Grieb, 2015, S. 47f.). Die Schulsozialarbeit wird teilweise zu spät und von den Lehrpersonen nur zögerlich oder selten involviert. Informationsdefizite, Aufgabenüberschneidungen, unterschiedliche Organisationsstrukturen und berufskulturelle Unterschiede sind mögliche Gründe für solche Kooperationsdefizite (Olk, 2005). Vor diesem Hintergrund stellt sich die Frage, welche Merkmale die interdisziplinäre Zusammenarbeit zwischen Schulsozialarbeitenden und Lehrpersonen im kaum erforschten Deutschschweizer Kontext auszeichnen. 


\section{Methodisches Vorgehen}

\section{Datengrundlage}

Die hier vorgestellten Forschungsergebnisse basieren auf dem vom Schweizerischen Nationalfonds finanzierten Forschungsprojekt «Kooperationsformen und Nutzungsstrukturen in der Schulsozialarbeit». ${ }^{1}$ Darin untersuchen die Pädagogische Hochschule Bern und die Berner Fachhochschule die Zusammenarbeit zwischen Schulsozialarbeitenden, Schulleitungen, Lehrpersonen und schulexternen Fachstellen in allen deutschsprachigen Schulen der Schweiz mit Schulsozialarbeit. Bei den folgenden Ergebnissen handelt es sich um eine deskriptive Teilauswertung der Befragungsdaten von Schulsozialarbeitenden aus den vier Kantonen Bern, Basel-Landschaft, Basel-Stadt und Wallis, in denen die Befragung abgeschlossen ist.

Die Schulsozialarbeitenden wurden online zu ihrer Einschätzung zur $\mathrm{Zu}-$ sammenarbeit mit den Lehrpersonen befragt. Von den 175 kontaktierten Schulsozialarbeitenden haben 173 an der Studie teilgenommen, was einer Rücklaufquote von 98.9 Prozent entspricht. ${ }^{2}$ Da es sich um eine Vollerhebung der Schulsozialarbeitenden in vier Kantonen handelt, die Rücklaufquote hoch und die Zahl der fehlenden Werte klein ist ( 0.6 bis 2.3\%), sind die Daten insgesamt von guter Qualität und hoher Aussagekraft.

Von den 173 Schulsozialarbeitenden in den vier Kantonen sind 65.3 Prozent Frauen und 34.7 Prozent Männer. Damit ist der Männeranteil etwa 10 Prozent höher als in anderen Arbeitsfeldern der Sozialen Arbeit (vgl. Kessler \& Schmid, 2012). Der mittlere Beschäftigungsgrad der befragten Personen beträgt 40 bis 79 Prozent. Mit 39.4 Prozent der befragten Schulsozialarbeitenden weisen die meisten weniger als drei Jahre Berufserfahrung auf. Weitere 35.9 Prozent geben an, zwischen drei bis weniger als sieben Jahre in der Schulsozialarbeit tätig zu sein. Der Anteil mit sieben und mehr Jahren Berufserfahrung beträgt 24.7 Prozent. Die meisten Schulsozialarbeitenden $(63.2 \%)$ sind für ein bis zwei Schulhäuser zuständig und mehrheitlich (86\%) zu fixen Zeiten im Schulhaus anwesend. 84.3 Prozent der Befragten verfügen über einen Arbeitsplatz in den jeweiligen Schulhäusern. Die Verteilung der Schulsozialarbeitenden über ausgewählte persönliche und organisatorische Merkmalsvariablen ist der Tabelle 1 zu entnehmen.

Siehe dazu: http://p3.snf.ch/project-156642

2 Mit einem aufwändigen Datenerhebungsverfahren wurden alle Schulsozialarbeitenden kontaktiert, die im Zeitraum zwischen August und Dezember 2016 berufstätig waren. Da es sich bei der Schulsozialarbeit um ein sehr dynamisches Arbeitsfeld handelt, kann es sein, dass es heute mehr als 175 Schulsozialarbeitende in den vier Kantonen gibt. 
Tabelle 1: Persönliche und organisatorische Merkmale der befragten Schulsozialarbeitenden

\begin{tabular}{lrr}
\hline & absolut $^{\text {a }}$ & in \% \\
\hline $\begin{array}{l}\text { Persönliche Merkmale: } \\
\text { nach Geschlecht }\end{array}$ & & \\
$\quad$ Weiblich & 111 & 65.3 \\
$\quad$ Männlich & 59 & 34.7 \\
nach Berufserfahrung & & \\
$\quad$ Weniger als 1 Jahr bis weniger als 3 Jahre & 67 & 39.4 \\
3 bis weniger als 7 Jahre & 61 & 35.9 \\
7 und mehr Jahre & 42 & 24.7 \\
nach Beschäftigungsgrad & & \\
0-39\% & 15 & 8.8 \\
$40-79 \%$ & 120 & 70.6 \\
$80 \%$ und höher & 35 & 20.6 \\
Organisatorische Merkmale: & & \\
Anzahl Schulhäuser im Zuständigkeitsbereich & 108 & 63.2 \\
1 bis 2 & 32 & 18.7 \\
3 bis 4 & 31 & 17.9 \\
5 und mehr & & \\
Anwesenheit zu festen Zeiten & 148 & 86.0 \\
Ja & 24 & 14.0 \\
Nein & & \\
Arbeitsplatz am Schulhaus & 145 & 84.3 \\
Ja & 27 & 15.7 \\
Nein & &
\end{tabular}

${ }^{a}$ Die Abweichungen zum Total von 173 kommen aufgrund von 1 bis 3 fehlenden Werten zustande.

\section{Operationalisierung der interdisziplinären Zusammenarbeit}

Die fünf Komponenten innerschulischer interdisziplinärer Zusammenarbeit gemäss dem Modell von Bronstein $(2002 ; 2003)$ wurden mit jeweils vier Frageitems auf einer Antwortskala von eins (,trifft überhaupt nicht zu“) bis fünf (,trifft voll zu“) gemessen. Interdependenz zielt dabei auf die gegenseitige Abhängigkeit und Unterstützung der beteiligten Professionen und wurde beispielsweise mit dem Item „Lehrpersonen und Schulsozialarbeitende unterstützen sich gegenseitig in der Arbeit mit den Schülerinnen und Schülern “ gemessen. Die zweite Dimension erfasst, inwiefern Schulsozialarbeitende und Lehrkräfte gemeinsame Ziele verfolgen und Verantwortung für die Zielerreichung übernehmen (Beispielitem: „Lehrpersonen und Schulsozialarbeitende teilen gemeinsame Zielvorstellungen"). Unter Flexibilität versteht das Modell die Aufgeschlossenheit und das Verständnis für Angehörige anderer 
Berufsgruppen. Diese Dimension wurde u.a. mit dem Item „Lehrpersonen und Schulsozialarbeitende sind offen gegenüber neuen Ideen, wie den Schülerinnen und Schülern geholfen werden kann " gemessen. Zudem bedingt die interdisziplinäre Zusammenarbeit neue Strategien, Programme und Strukturen für gemeinsame Aktivitäten. Um dies zu messen, wurden die Schulsozialarbeitenden beispielsweise um ihre Einschätzung zu folgendem Item gebeten: „Durch die Zusammenarbeit mit Lehrpersonen werden den Schülerinnen und Schülern neue Formen der Unterstützung angeboten ". Schliesslich erfordert eine interdisziplinäre Zusammenarbeit die kontinuierliche Überprüfung und Reflexion gemeinsamer Arbeitsprozesse, was mit dem Beispielitem „Lehrpersonen und Schulsozialarbeitende verwenden Feedbacks, um die Effektivität der Teamarbeit zu steigern " gemessen wurde.

\section{Ergebnisse}

Die Abbildung 2 zeigt den Median ${ }^{3}$ - für alle fünf Komponenten der interdisziplinären Zusammenarbeit. Zudem verweisen das erste und dritte Quartil auf die Streuung in den Antworten der Schulsozialarbeitenden, wobei das erste Quartil $\left(\mathrm{x}_{\mathrm{Q} 1}\right)$ für die kritischeren Angaben der untersten 25 Prozent und das dritte Quartil ( $\left.\mathrm{x}_{\mathrm{Q} 3}\right)$ für die positiveren Angaben der obersten 25 Prozent der befragten Schulsozialarbeitenden steht.

Insgesamt verweisen die Ergebnisse auf ein eher positives Bild der interdisziplinären Zusammenarbeit, da der Median für vier der fünf Komponenten über dem mittleren Wert von drei liegt. Gleichzeitig ist zu beobachten, dass es deutliche Unterschiede bei den Einschätzungen der befragten Schulsozialarbeitenden gibt.

3 Der Median $\mathrm{x}_{\text {Med }}$ ist ein Mass der zentralen Tendenz. Er gibt diejenigen Ausprägungen an, welche die nach Grösse geordneten Daten in der Mitte teilen. Dadurch sind mindestens $50 \%$ der Daten kleiner/gleich und mindestens 50\% grösser/gleich $\mathrm{x}_{\text {Med }}$ (Jann, 2005, S. 33). 


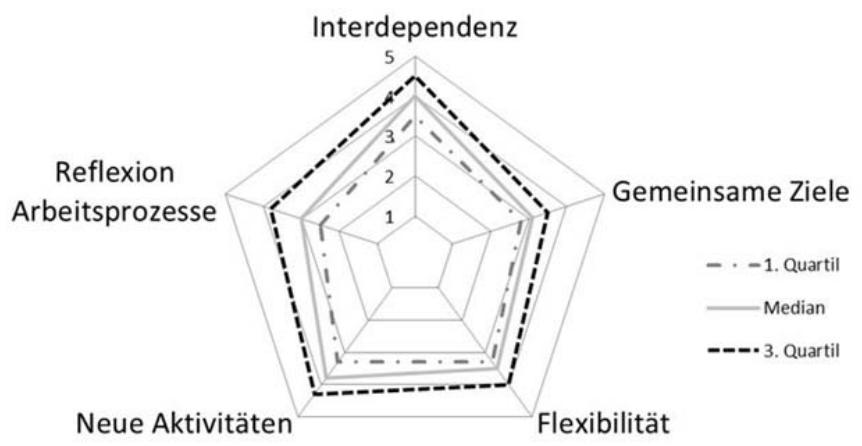

Abbildung 2: Merkmale der interdisziplinären Zusammenarbeit

Die gegenseitige Abhängigkeit und Unterstützung von Schulsozialarbeitenden und Lehrpersonen (Interdependenz) wird mit einem Median $\left(\mathrm{x}_{\mathrm{Med}}\right)$ von 4.0 insgesamt positiv eingeschätzt. Die Hälfte der Schulsozialarbeitenden bewertet diese Komponente mit einem Indexwert zwischen 3.5 und 4.5. Ein Viertel der Befragten ist diesbezüglich optimistischer $\left(\mathrm{x}_{\mathrm{Q} 3} \geq 4.5\right)$ und die übrigen 25 Prozent finden, dass die Interdependenz mit den Lehrkräften nur teilweise oder nicht gegeben ist $\left(\mathrm{x}_{\mathrm{Q} 1} \geq 3.5\right)$.

Am kritischsten wird die Reflexion gemeinsamer Arbeitsprozesse bewertet $\left(\mathrm{x}_{\mathrm{Med}}=3\right)$. Gemäss Angaben der Schulsozialarbeitenden erfolgt der gegenseitige Austausch über Arbeitsprozesse und deren Überprüfung nur teilweise. Zudem wird diese Komponente sehr unterschiedlich beurteilt. Das kritischste Viertel der Befragten gibt an, dass die gemeinsamen Arbeitsprozesse mit Lehrpersonen eher nicht reflektiert und überprüft werden $\left(\mathrm{x}_{\mathrm{Q} 1} \geq 2.5\right)$. Für das obere Viertel ist es dagegen eher oder ganz zutreffend, dass man sich zwecks Verbesserung der Zusammenarbeit regelmässig austauscht und bestehende Prozesse überprüft $\left(\mathrm{x}_{\mathrm{Q} 3} \geq 3.8\right)$.

Die übrigen Aspekte der interdisziplinären Zusammenarbeit werden als mittelmässig bis eher gut bewertet. Die Komponente Flexibilität, welche die Aufgeschlossenheit und das Verständnis für Angehörige anderer Berufsgruppen misst, weist einen Median von 3.5 auf. Der mittlere Wert für die Fragen $\mathrm{zu}$ den neuen Aktivitäten von Schulsozialarbeitenden und Lehrpersonen $\left(\mathrm{x}_{\mathrm{Med}}=3.8\right)$ liegt leicht über diesem Wert. Gleichzeitig ist hier der Abstand zwischen dem ersten und dritten Quartil grösser. Dies verweist darauf, dass es an den verschiedenen Standorten relativ grosse Unterschiede gibt, wie 
stark sich Schulsozialarbeitende und Lehrpersonen für gemeinsame Aktivitäten, Programme und Projekte engagieren. Jeweils 25 Prozent der befragten Schulsozialarbeitenden geben an, dass die Flexibilität und neue gemeinsame Aktivitäten nur teilweise oder gar nicht gegeben sind $\left(\mathrm{x}_{\mathrm{Q} 1} \geq 3.3\right)$. Insgesamt werden die Komponenten Flexibilität und gemeinsame Aktivitäten positiver eingeschätzt als das Vorhandensein gemeinsamer Zielvorstellungen $\left(\mathrm{x}_{\mathrm{Med}}=\right.$ $3.1)$.

\section{Diskussion und Fazit}

Mit der Einführung der Schulsozialarbeit stellen sich wichtige Fragen zur Ausgestaltung von Zusammenarbeitsformen zwischen Schulsozialarbeitenden, Lehrpersonen und anderen Akteurinnen und Akteuren. Dieser Beitrag ging von den fünf Merkmalen interdisziplinärer Zusammenarbeit nach Bronstein (2003) aus und beschrieb, wie diese in der Zusammenarbeit zwischen Schulsozialarbeitenden und Lehrpersonen nach der Einschätzung der Schulsozialarbeitenden ausgeprägt sind.

Die Ergebnisse aus vier Kantonen zeigen, dass die interdisziplinäre $\mathrm{Zu}-$ sammenarbeit von den Schulsozialarbeitenden insgesamt eher positiv bewertet wird, in Bereichen wie der regelmässigen Überprüfung gemeinsamer Arbeitsprozesse oder der Einigung auf gemeinsame Ziele aber Optimierungspotential besteht. Ausserdem wird ersichtlich, dass die Zusammenarbeit in rund einem Viertel der Schulen nicht intensiv gepflegt wird. Dies ist daran zu erkennen, dass die fünf Dimensionen interdisziplinärer Zusammenarbeit von den befragten Schulsozialarbeitenden höchstens mittelmässig eingeschätzt wurden. Strukturelle und berufskulturelle Unterschiede sowie Informationsdefizite über die Aufgaben und Verantwortlichkeiten der jeweils anderen Profession können Gründe dafür sein (Bronstein, 2003; Olk, 2005). Wir schliessen daraus, dass die strukturelle Basis der Zusammenarbeit in vielen Schulen gestärkt werden sollte. Für die Überwindung von Kooperationsbarrieren braucht es angemessene Strukturen und Gefässe, in deren Rahmen sich die Beteiligten regelmässig über Herausforderungen, Arbeitsprozesse und Zielsetzungen verständigen und gegebenenfalls auf Anpassungen einigen können (Bronstein, 2003; Mellin, Bronstein \& Anderson-Butcher, 2010). Die relativ guten Werte hinsichtlich Flexibilität, Kompromissbereitschaft und gegenseitiger Unterstützung sind gute Voraussetzungen für den Aufbau solcher Strukturen und Gefässe.

Die Ergebnisse der Studie widerspiegeln die Sicht der Schulsozialarbeitenden. Diese Perspektive ist wichtig, weil Schulsozialarbeitende in einem vergleichsweise jungen Berufsfeld der Sozialen Arbeit tätig sind und sich auf «institutionellem Fremdgebiet» bewegen. Sie arbeiten am Ort der Schule und 
mit Lehrkräften zusammen, die in Überzahl vertreten sind. Die Perspektive der Lehrpersonen, Schulleitungen oder der Speziallehrkräfte wurde in diesem Beitrag dagegen nicht abgebildet. Auch die Ursachen für die beobachteten Unterschiede hinsichtlich der interdisziplinären Zusammenarbeit konnten im Rahmen dieser Teilauswertung nicht geprüft werden. Zukünftige Studien sollten deshalb die Erklärungskraft verschiedener Einflussfaktoren prüfen, Zusammenarbeitsformen zwischen mehreren Berufsgruppen untersuchen und dabei die Sicht mehrerer Berufsgruppen berücksichtigen. Insbesondere ist interessant zu untersuchen, inwiefern und wo sich die Einschätzungen der verschiedenen Berufsgruppen decken. Diese und weitere Aspekte sind Gegenstand weiterer Untersuchungen im Rahmen des Gesamtprojekts „Kooperationsformen und Nutzungsstrukturen in der Schulsozialarbeit" (www.phbern.ch/ schulsozialarbeit).

\section{Literatur}

Baier, F. \& Heeg, R. (2011). Praxis und Evaluation von Schulsozialarbeit: Sekundäranalysen von Forschungsdaten aus der Schweiz. Wiesbaden: VS Verlag für Sozialwissenschaften.

Bronstein, L. (2002). Index of interdisciplinary collaboration. Social Work Research, 26(2), 113-126.

Bronstein, L. (2003). A model for interdisciplinary collaboration. Social Work, 48(3), 297-306.

Darlington, Y., Healy, K. \& Feeney, J. A. (2010). Approaches to assessment and intervention across four types of child and family welfare services. Children and Youth Services Review, 32(3), 356-364.

Drilling, M. (2009). Schulsozialarbeit: Antworten auf veränderte Lebenswelten (4. Aufl.). Bern: Haupt.

Hafen, M. (2005). Probleme und Entwicklungsmöglichkeiten der Sozialen Arbeit in der Schule. SozialAktuell, Nr. 13/2005: 6-9.

Higham, J. \& Yeomans, D. (2010). Working together? Partnership approaches to 1419 education in England. British Educational Research Journal, 36(3), 379-401.

Jann, B. (2005). Einführung in die Statistik. München: Oldenbourg Wissenschaftsverlag.

Kessler, R. \& Schmid, G. (2012). „Das ungenutzte Potential“. SozialAktuell. Fachzeitschrift für Soziale Arbeit, Nr. 1/2012, 10-12.

Mellin, E., Anderson-Butcher, D. \& Bronstein, L. (2011). Strengthening interprofessional team collaboration: potential roles for school mental health professionals. Advances in School Mental Health Promotion, 4(2), 51-61.

Mellin, E., Bronstein, L. \& Anderson-Butcher, D. (2010). Measuring interprofessional team collaboration in expanded school mental health: Model refinement and scale development. Journal of Interprofessional Care, 24(5), 514-523.

Olk, T. (2005). Kooperation zwischen Jugendhilfe und Schule. In Sachverständigenkommission Zwölfter Kinder- und Jugendbericht (Hrsg.), Band 4: Kooperationen 
zwischen Jugendhilfe und Schule (S. 9-100). München: Verlag Deutsches Jugendinstitut.

Olk, T. \& Speck, K. (2012). Kooperation von Jugendhilfe und Schule. In W. Thole (Hrsg.), Grundriss Soziale Arbeit (S. 355-360). Wiesbaden: VS Verlag für Sozialwissenschaften.

Olk, T., Bathke, G. \& Hartnuss, B. (2000). Jugendhilfe und Schule: Empirische Befunde und theoretische Reflexionen zur Schulsozialarbeit. Weinheim: Juventa.

Pfiffner, R. \& Grieb, M. (2015). Evaluation Schulsozialarbeit Steffisburg. Bern: Berner Fachhochschule.

Stratz, H. \& Wiklund, S. (2013). One for all or all for one? The collaboration of Swedish personal social services with regard to lone mothers on social assistance. British Journal of Social Work, 45(2), 549-567. 


\title{
„Die wissen gar nicht, was wir alles machen“. Befunde zu multiprofessioneller Kooperation im Zuge der Einführung von Tagesschulen in der Stadt Zürich
}

\author{
Emanuela Chiapparini, Kadrie Selmani, Christa Kappler, \\ Patricia Schuler
}

In zahlreichen Studien gelten multiprofessionelle Kooperationen als eine zentrale Einflussgrösse für die Optimierung organisatorischer Abläufe in Regelschulen und besonders in Tagesschulen (Fischer et al., 2013; Olk, Speck \& Stimpel, 2011). Kooperationen mit anderen Professionen stellen für Lehrkräfte eine zeitliche Entlastung dar, stärken das eigene Professionsverständnis, werden als förderliche Lernräume betrachtet oder sollen gelingende Schulentwicklung, z.B. beim Einführen von Tagesschulen, sichern.

Gleichzeitig werden professionelle Kooperationen als Spannungsfeld (Merten \& Kaegi, 2015) beschrieben und hohe Erwartungen an eine gelingende Kooperationskultur von schulpädagogischen, sozialpädagogischen und anderen Fachkräften in der Schule gestellt.

Hier knüpft der vorliegende Beitrag an, in welchem auf die Kooperation von Lehrkräften und sozialpädagogischen Fachkräften ${ }^{1}$ fokussiert wird. Auch in der Schweiz werden zunehmend Tagesschulen eingeführt und die Kooperation zwischen Lehrkräften und sozialpädagogischen Fachpersonen konzeptionell verankert und gefordert wie z.B. im Zürcher Tagesschulmodell (Stadt Zürich, 2017).

1 Die Begriffe „Soziale Arbeit“ und „Sozialpädagogik“ werden im vorliegenden Beitrag als konvergent (Lambers, 2013) verstanden.

Zudem umfasst die Bezeichnung „sozialpädagogische Fachkräfte“ alle Personen, die sozialpädagogisch qualifiziert sind. Hierzu zählen Fachkräfte, die einen Bachelorabschluss in Sozialer Arbeit an einer Fachhochschule oder höheren Fachschule vorweisen, und weitere Fachkräfte mit sozialpädagogischem Wissen.

In der Schweiz sind darüber hinaus für die sozialpädagogischen Fachkräfte die Bezeichnungen Fachperson Soziale Arbeit oder Fachpersonen Betreuung, Hortner/-innen oder schlicht Betreuungspersonen üblich (vgl. AvenirSocial 2011). Die Verbreitung des Betreuungsbegriffs hängt ebenfalls mit der verbreiteten Berufsausbildung „Fachpersonen Betreuung“ zusammen. Diese Fachkräfte bringen sozialpädagogisches Wissen mit und sind im Tagesschulbereich stark vertreten.

Schliesslich zeigen diese Ausführungen die Heterogenität und Länderspezifik der Bezeichnungen im Fachgebiet Soziale Arbeit auf. 
Während in der Schulforschung in Deutschland bereits einzelne Studien $\mathrm{zu}$ förderlichen Bedingungen für Kooperationen zwischen schulischen und sozialpädagogischen Fachkräften vorliegen, wurden die Kooperationsformen von sozialpädagogischen Fachkräften im schweizerischen Schulsystem ungenügend empirisch untersucht. Ausgehend von Konzepten der ,multiprofessionellen“, ,inter-/intraprofessionellen“ und „transprofessionellen“ Kooperation und der Klärung des professionstheoretischen Verständnisses werden in diesem Beitrag empirische Befunde aus dem laufenden SNF-Forschungsprojekt zu pädagogischen Zuständigkeiten an Tagesschulen (AusTEr, 2016) vorgestellt. Dazu werden die Fragen aufgegriffen und erkundet, wie Lehrkräfte und sozialpädagogische Fachkräfte die Zusammenarbeit unter sich wahrnehmen und welche daraus folgenden Bezüge zum Professionalisierungsverständnis sich präsentieren.

\section{Tagesschulentwicklungen in der Schweiz}

Tagesschulen gewinnen als institutionalisierte Formen von öffentlicher Bildung und Erziehung immer mehr an Bedeutung in der Schweiz (Chiapparini et al., 2016). Besonders deutlich zeichnet sich dies in der Schulentwicklung der Stadt Zürich ab, welche als erste Schweizer Gemeinde und im Rahmen des Projekts „Tagesschule 2025“ flächendeckend und schrittweise die bestehenden Regelschulen in gebundene Tagesschulen überführt. Anders als im Kanton Bern, wo mit dem Begriff „Tagesschule“ das freiwillige Hortangebot im Schulhaus gemeint ist, zeichnen sich Tagesschulen in der Stadt Zürich dadurch aus, dass alle Schülerinnen und Schüler an mindestens drei Tagen verbindlich ausserunterrichtliche Angebote im und ums Schulhaus nutzen (wie beispielsweise Mittagstisch oder ,erweiterte Lernangebote“) sowie weitere fakultative Förder- und Freizeitangebote nutzen. Dieses Schulmodell orientiert sich im Gegensatz zur klassischen Schulpädagogik an einem erweiterten Bildungs- und Erziehungsbegriff, sodass Bildung und Erziehung nicht nur im Unterricht, sondern ebenso in ausserunterrichtlichen Angeboten stattfindet. Bereits konzeptionell sind multiprofessionelle Kooperationen (Fischer et al., 2013; Olk, Speck \& Stimpel, 2011) in Tagesschulmodellen angesetzt, wobei diese zumindest im Schweizer Kontext theoretisch und empirisch ungenügend fundiert sind. Vertretende der Bildungsdirektion des Kantons Zürich (Bildungsdirektion Kanton Zürich, 2017) schlagen hierzu eine pragmatische und bildungsgesetzorientierte Perspektive für alle politischen Gemeinden des Kantons Zürich vor, indem die Verzahnung von Schulpädagogik und Sozialer Arbeit auf struktureller, personeller, pädagogischer und räumlicher Ebene differenziert reflektiert wird. 


\section{Theoretischer Rahmen und Forschungstand}

Grundsätzlich werden Kooperationen auf institutioneller oder auf personeller Ebene entweder als Strukturmerkmal oder als Handlungsmaxime unterschieden (z.B. Merten \& Kaegi, 2015). Richtet sich der Fokus auf die personelle Ebene, wie dies bei dem vorliegenden Beitrag der Fall ist, lassen sich drei Formen unterscheiden, die allerdings keine einheitliche Verwendung finden: a) multiprofessionelle, b) inter-/intraprofessionelle und c) transprofessionelle Kooperationen.

a) Mit „multiprofessioneller Kooperation“ (z.B. Fischer et al., 2013) wird im Kontext Schule die Zusammenarbeit zwischen inner- und ausserschulischen Fachkräften bezeichnet. $\mathrm{Zu}$ den innerschulischen Fachkräften zählen Lehrkräfte, sozialpädagogische Fachkräfte oder Heilpädagoginnen und Heilpädagogen, zu den ausserschulischen Fachkräften beispielsweise Trainerinnen und Trainer eines Sportvereins oder Jugendarbeiterinnen und Jugendarbeiter eines Jugendtreffs. Hierbei handelt es sich um eine offene und voraussetzungslose Kooperation, sodass ein Nebeneinander oder eine Koordinationsfunktion der Fachkräfte möglich ist.

b) Demgegenüber wird mit der Bezeichnung ,,inter- und intraprofessionelle Kooperation“ (z.B. Dizinger, 2015) erstens impliziert, ob es sich um eine Kooperation unter unterschiedlichen oder unter gleichen Fachkräften handelt. Zweitens ist die Richtung festzuhalten, aus welcher die Kooperation stattfinden soll, z.B. aus der Sicht einer Lehrkraft und einer weiteren an der Schule pädagogisch tätigen Person oder aus der Sicht der sozialpädagogischen Fachkräfte und Lehrkräfte. Drittens werden Synergien in der Arbeit erkannt und Ziel ist es, diese zu nutzen, um Arbeitsprozesse optimal zu gestalten.

c) Der Begriff der „transprofessionellen Kooperation“ (z.B. Chiocchio \& Richer, 2015) ist im Gesundheitswesen stark verbreitet, womit in einer Projektgruppe die Fachkräfte ihre üblichen Rollen vorerst zurückstellen und professionelles Wissen der anderen Fachgruppe in der Zusammenarbeit übernehmen und sich aneignen. Transprofessionelle Kooperation ist vor allem in kurzfristigen Projekten üblich (ebd.).

Obwohl in der Tagesschuldebatte die multiprofessionelle Kooperation zwischen Lehrkräften und sozialpädagogischen Fachkräften in den Vordergrund rückt, weisen gewisse Definitionen ebenso auf inter- und transprofessionelle Kooperationen zwischen beiden Berufsgruppen hin. Wenn beispielsweise als Kooperation die Arbeitsteilung sowie der intensive Austausch und die Konstruktion von gemeinsamem Wissen intendiert wird (Kamski, 2011) und damit Synergien angestrebt werden, handelt es sich gemäss der oberen Definiti- 
on eher um interprofessionelle Kooperationen. Wenn sich zudem das professionelle Handlungssetting und die Aufgaben beider Professionen entsprechend erweitern (Breuer \& Reh, 2010), wäre abzuwägen, inwiefern ein transprofessionelles Verständnis von Kooperation vorliegt.

Professionelle Kooperationen basieren zudem auf den jeweiligen „Berufskulturen“ (Terhart, 1997), welche sich aufgrund von dahinterstehenden gesellschaftlichen Institutionen durch je spezifische gesellschaftliche Aufträge, Handlungslogiken, Arbeitsprinzipien und professionelle Entwicklungsgeschichten (Paseka, 2011) unterscheiden.

Im vorliegenden Beitrag ist die Profession als Handlungsstruktur (Nittel, 2011) und nicht als Zustand zu verstehen. Folglich geht es bei der professionellen Kooperation um eine umfassende und reflexive Form, die eine multiprofessionelle Arbeitsbeziehung über einen gesamten Arbeitsprozess hindurch voraussetzt. Dieses Verständnis von multiprofessioneller Kooperation grenzt sich von einem relativ konflikt- und reflexionsfreien Nebeneinander von professionsspezifischen Handlungsorientierungen ab.

Allerdings fokussieren gegenwärtige Studienbefunde in Deutschland und der Schweiz vor allem auf letztgenanntes Verständnis von multiprofessioneller Kooperation, indem deren Gelingensfaktoren nachgezeichnet werden. Erfolgreiche multiprofessionelle Kooperation stellt sich eher ein, wenn beispielsweise der Nutzen durch die Kooperierenden (z.B. Olk, Speck \& Stimpel, 2011) wahrgenommen wird oder wenn Statuserwartungen und -zuschreibungen oder Fragen der Verbindlichkeit sowie der Ressourceneinbringung geklärt sind (vgl. Santen \& Seckinger, 2003). Zudem spielen optimale strukturelle Bedingungen eine zentrale Rolle, wie beispielsweise ausreichende materielle, personelle und räumliche Ressourcen, festgelegte Konzepte der Zusammenarbeit oder die Offenheit der Lehrkräfte für Betreuungsarbeit (Holtappels et al., 2011).

In der bisher einzigen Studie zum Thema aus der Schweiz (Schüpbach, Jutzi \& Thomann, 2012) werden vier zentrale Kriterien für gelingende Kooperation auf die Kommunikationsebene bezogen: a) reger Informationsfluss und -austausch, b) gute Kommunikationskultur, c) Offenheit gegenüber der Zusammenarbeit und d) persönliche Kontakte.

Zwei der wenigen Studien im deutschsprachigen Raum, die Kooperationen auf das Professionsverständnis fokussieren, sind die qualitative ProfKoop-Studie (Olk, Speck \& Stimpel, 2011; Speck et al., 2011) und die Studie von Böhm-Kasper, Dizinger \& Heitmann (2013): Insbesondere Lehrkräfte haben eine pragmatische Vorstellung von Kooperation, die in einer „losen Form" besonders tragfähig erscheint. Des Weiteren halten die Autoren fest, dass Spannungen der unterschiedlichen Berufskulturen insbesondere bei konkreten Anlässen in der Praxis ausgetragen werden und ein Eingriff in die Autonomie der jeweiligen Profession nicht als förderlich erachtet wird. 
Vor dem Hintergrund der oben gemachten professionstheoretischen Skizzierung, in welcher der Fokus auf Handlungsstrukturen gesetzt wird, sollen im Folgenden die Erwartungen der Professionen der Lehrkräfte und der sozialpädagogischen Fachkräfte untersucht werden, wo sie ihre pädagogischen Verantwortungen sehen. Parallel dazu geht es in der empirischen Frage darum, wie die Lehrkräfte und sozialpädagogischen Fachkräfte die explizite und implizite Kooperation zwischen ihren beiden Professionen schildern.

\section{Anlage der Studie}

Der vorliegende Beitrag knüpft an das vom Schweizerischen Nationalfonds geförderte Forschungsprojekt AusTEr (Chiapparini et al., 2016) an, welches mit einem professionstheoretischen Blick Aushandlungsprozesse von pädagogischen Zuständigkeiten an Tagesschulen im Spannungsfeld öffentlicher Erziehung erörtert.

$\mathrm{Zu}$ zwei Messpunkten werden vier Fälle von sich neu konstituierenden Tagesschulen in der Stadt Zürich untersucht (Cross Case Study). Da eine „Theorie der Ganztagsbildung“ (Coelen \& Stecher, 2014) fehlt, wurde ein qualitativer Zugang gewählt. Zudem erlaubt es dieser Zugang, den gedeuteten Ist-Zustand der unterschiedlichen Beteiligten an einer Ganztagesschule differenziert zu berücksichtigen. In der ersten Erhebungsphase wurden 55 Einzel- und Gruppeninterviews mit narrativen Passagen (Schütze, 1983; Bohnsack, 1999) geführt, um die subjektiven sowie kollektiven Sichtweisen möglichst aller an der Tagesschule Tätigen (schulische und sozialpädagogische Fachkräfte, ausserunterrichtliche Angebote von Externen, Erziehungsberechtigte sowie Schülerinnen und Schüler) zu berücksichtigen. Das Gesprächsmaterial wurde vollständig transkribiert und mit der Grounded Theory (Strauss \& Corbin, 1997) auf gemeinsame und kontrastierende Deutungsmuster hin analysiert. Der skizzierte qualitative Zugang ermöglicht es, aus den Erzählungen explizite und implizite Vorstellungen und Deutungsmuster zu rekonstruieren und die Handlungsroutinen und Sichtweisen der handelnden Personen abzubilden, welche latent in den Aussagen enthalten sind und von ihnen nicht explizit geäussert werden. Die anschliessend vorgestellten Befunde berücksichtigen die Gespräche mit den 16 Lehrkräften und 15 sozialpädagogischen Fachkräften.

Da der Begriff „Kooperation“ von einem normativen und affirmativen Charakter geprägt ist, indem Kooperationen grundsätzlich als wünschenswert erachtet werden, wird im Forschungsprojekt ein offener Zugang gewählt: Kooperation wird als Rahmenbedingung des Handelns verstanden, die nicht von vornherein als wünschenswert einzustufen ist. 
Des Weiteren bildet die Gruppe der sozialpädagogischen Fachkräfte eine bezüglich Ausbildungszertifikaten heterogene Gruppe, welche Betreuungsassistentinnen und -assistenten ohne pädagogische Ausbildung, Fachangestellte Betreuung mit einer Berufsausbildung und „Hortleiterinnen“, die eine Fachhochschule oder höhere Fachhochschule der Sozialen Arbeit besucht haben, umfasst (vgl. in der Einleitung Fussnote 1). Anders als bei einem professionstheoretisch eng geführten Verständnis, bei dem eine Profession nur mit einem Hochschulabschluss und als Status verstanden wird, wird in dieser Untersuchung der Blick auf die Handlungen und die Professionalität (Nittel, 2011) gerichtet, sodass die sozialpädagogischen Fachkräfte mit einer Berufslehre ebenfalls eine multiprofessionelle Kooperation eingehen können.

\section{Befunde}

\subsection{Tendenzen der Zusammenarbeit und Koordination}

Zunächst zeigen die Befunde einige Tendenzen zu expliziter und impliziter Kooperation zwischen den Lehrkräften und den sozialpädagogischen Fachkräften in den untersuchten Schulhäusern. Hierbei ist es wichtig darauf hinzuweisen, dass der Begriff der „Kooperation“ mehrheitlich in der Fachsprache verwendet wird, die Interviewteilnehmenden jedoch von „Zusammenarbeit" sprechen, weshalb dieser Begriff auch in diesem Unterkapitel Verwendung findet. Zudem soll an dieser Stelle darauf hingewiesen werden, dass die Lehrkräfte und die sozialpädagogischen Fachkräfte ausserunterrichtliche Angebote in den hier vorliegenden Untersuchungsfällen mit dem Begriff „Hort“ gleichsetzen, in welchem unterschiedliche Angebote stattfinden.

Im Besonderen werden von beiden Professionen die ungünstigen örtlichen und zeitlichen Rahmenbedingungen erwähnt, welche eine Zusammenarbeit erschweren.

Eine weitere wichtige Tendenz zeigt sich darin, dass die genannte $\mathrm{Zu}$ sammenarbeit meist in Form von organisatorischem und administrativem Austausch und Absprachen dargestellt wird.

Die analysierten Daten zeigen kaum pädagogische oder inhaltliche $\mathrm{Zu}$ sammenarbeit, vielmehr lässt sich ein Nebeneinander im Sinne einer Koordination der beiden Arbeitsbereiche erkennen (unterrichtliche und ausserunterrichtliche Angebote). Ein Ausnahmebeispiel nennt folgende sozialpädagogische Fachperson, die eine konkrete Situation der Zusammenarbeit der beiden Professionen wie folgt schildert und diese als grossen Gewinn erachtet: 
«Ehm ich merke halt, dass dann auch Themen aus dem Bereich Unterricht in die Betreuung kommen. Also da wird mal miteinander ein Quiz gemacht, ein Geografie-Quiz oder irgendetwas, ja es lebt zwischen Unterricht und Betreuung.» (Schulhaus D, sozialpädagogische Fachkraft 2, 124)

Die Analyse der Daten offenbart zudem eine tendenzielle soziale Erwünschtheit der Zusammenarbeit, dass diese grundsätzlich positiv besetzt ist. Das ist in den Aussagen beider Professionen wiederzufinden: So sprechen Lehrkräfte explizit vom ,guten Austausch“ (Schulhaus A, Lehrkraft 1,111) und sozialpädagogische Fachkräfte davon, dass es wichtig sei, ,den Kindern [zu] vermitteln, dass alle an einem Strang ziehen an der Tagesschule (...)“ (Schulhaus C, sozialpädagogische Fachkraft 1, 77). Eine weitere Lehrkraft betont: „Mir ist es auch wichtig eben mit dem Hort zusammenzuarbeiten“ (Schulhaus A, Lehrkraft 2, 34, 87). Besonders in Textstellen, die die Tendenz der normativen Erwartung widerspiegeln, lassen sich explizite Benennungen des Begriffs der Zusammenarbeit finden. Neben diesen wird die Zusammenarbeit vielmehr implizit erwähnt, indem Beschreibungen des Arbeitsalltages rekonstruiert werden.

In einem weiteren Schritt lassen sich aus den Befunden unterschiedliche Perspektiven zum Thema Zusammenarbeit herausarbeiten, woraus folgende zwei zentrale Deutungsmuster resultierten.

\subsection{Ungenügende Kenntnis über den Arbeitsbereich der anderen Profession}

Die sozialpädagogischen Fachkräfte bemängeln, dass die Lehrkräfte die sozialpädagogischen Aufgaben und Leistungen nur ungenügend kennen, wodurch diese wiederum eine geminderte Wertschätzung in ihren Tätigkeitsbereichen wahrnehmen. Dieses Muster geht besonders deutlich aus folgender Aussage hervor:

„(...) diese Unterschiede [zwischen Lehrkräften und sozialpädagogischen Fachkräften], die wirklich grundlegend sind, dass wir [sozialpädagogische Fachkräfte] die Kinder pflegen und wir unterrichten sie nicht, zum Teil nicht ganz respektiert (...) aber man [Lehrkräfte] ist sich eigentlich gar nicht bewusst, was man eigentlich sagt und was für Problematiken wir [sozialpädagogische Fachkräfte] auch ehm, tagtäglich mit was wir umgehen müssen (...)“ (Schulhaus A, sozialpädagogische Fachkraft 1, 65)

Gleichzeitig monieren die befragten Lehrkräfte desselben Schulhauses, dass wiederum die sozialpädagogischen Fachkräfte den Arbeitsort und Aufgabenbereich der Lehrkräfte nur ungenügend kennen würden. Folgendes Zitat bezieht sich auf Schulregeln im Verlauf der Einführung der Tagesschule: 
„Aber das beweist mir auch wie sie [sozialpädagogische Fachkräfte] sich vorher nicht mit uns auseinandergesetzt haben. Also sie haben gar nicht gewusst, was alles da ist" (Schulhaus A, Lehrkraft 2, 105)

Die Lehrkraft geht davon aus, dass sich die sozialpädagogischen Fachkräfte mit dem Tätigkeitsfeld der Lehrkräfte und den darin bestehenden Regeln auseinandergesetzt haben. Die implizite Erwartung besteht darin, dass sich die sozialpädagogischen Fachkräfte selbständig zu informieren und von dem auszugehen haben, was im Schul- und Unterrichtskontext besteht.

Obwohl beide Professionen der anderen Profession Unkenntnis vorwerfen, werden weitere Herausforderungen, wie beispielsweise der respektvolle Umgang, von der sozialpädagogischen Fachkraft thematisiert.

Dieses Muster der ungenügenden Kenntnis der anderen Profession und die Schieflage bezüglich gegenseitig wahrgenommenen Respekts sind in weiteren Interviewtextstellen in diesem und den anderen Schulhäusern wiederzufinden.

\subsection{Asymmetrische und einseitige Zusammenarbeit}

Ein weiterer Befund zeigt das Muster der asymmetrischen und einseitigen Zusammenarbeit.

Bereits in der konzeptionellen Verankerung der Tagesschule ist eine beschränkte Form der Zusammenarbeit vorgesehen, wenn beispielsweise Hausaufgaben nicht mehr wie bisher von den sozialpädagogischen Fachkräften, sondern nur noch von Lehrkräften angeboten werden können, oder wenn sozialpädagogische Fachkräfte zwar bei Schulausflügen willkommen sind, aber keine gemeinsame Organisation der beiden Professionen vorgesehen ist (z.B. Schulhaus A, sozialpädagogische Fachkraft 1, 59). Hier liegen nicht nur Kompetenzen der sozialpädagogischen Fachkräfte brach, ebenso werden vorhandene inhaltliche und organisationale Synergien nicht genutzt.

Weiter erwähnen mehrere sozialpädagogische Fachkräfte im Interview, dass in Sitzungen, zu denen sie neu seit der Tagesschule hinzukamen und in denen sie untervertreten sind, die Anliegen und Wünsche von Lehrkräften mehr Gewicht hätten. So würden zum Beispiel Problemlösungen bei der Kontaktaufnahme mit Eltern gesucht, wo sozialpädagogische Fachkräfte gar keine Probleme sehen, wie folgendes Zitat untermauert.

„Also wirklich gar nicht, wir haben wirklich alle neutral und einzeln sagen können, dass es [das „Problem“, Eltern zu kontaktieren] unsere Arbeit noch nie beeinträchtigt hat. (...) Also eben, das ist wie, dass die Lehrer manchmal Problematiken sehen, wo wir gar keine sehen und dann aber wie uns [dabei haben] wollen, und sie 
schaffen es dann natürlich auch, oder. Und dann wird das beschlossen und dann ist das so (...)“(Schulhaus A, sozialpädagogische Fachkraft 1, 69).

Diese Textstelle impliziert einerseits eine Einschränkung der Themensetzung in Sitzungen, auch wenn nach eigener Ansicht die Eigenposition sachlich und neutral kommuniziert wurde. Andererseits kommen implizit die unterschiedlichen Arbeitsformen beider Professionen zum Zuge, wie die als mehr oder weniger herausfordernd wahrgenommene Zusammenarbeit mit Eltern. Im Anschluss daran steht nicht die Frage im Raum, was allenfalls von den sozialpädagogischen Fachkräften in der Zusammenarbeit mit Eltern gelernt werden kann, sondern die nicht nachvollziehbare und nicht geteilte Themensetzung und das Hinnehmen der gefällten Entscheidung sind zentral. In anderen Aussagen derselben sozialpädagogischen Fachkraft oder auch von einigen Kolleginnen und Kollegen ist eine teilweise resignierte Haltung dokumentiert.

Die Tendenz einer einseitigen Zusammenarbeit zwischen Lehrkräften und sozialpädagogischen Fachkräften zeichnet sich exemplarisch an folgender Aussage einer Lehrkraft ab:

„[Der Hort] muss seine Rolle finden und sich in den Schulalltag eingliedern“ (Schulhaus A, Lehrkraft 1, 110).

Diese Lehrkraft wünscht sich eine einseitige Vernetzung. Ihrer Meinung nach ist es die Aufgabe der sozialpädagogischen Fachkräfte, sich in der Tagesschule einzugliedern.

Das Muster der asymmetrischen und einseitigen Zusammenarbeit zwischen sozialpädagogischen Fachkräften und Lehrkräften zeichnet sich nicht nur in gedeuteten Handlungen, sondern teilweise auch auf struktureller Ebene ab (bspw. im Konzept der Tagesschule), wie folgende Passage verdeutlicht:

„Es ist mal ein Betriebskonzept entwickelt worden für die gesamte Schule. Und ehm dort habe ich mitgearbeitet. (...) Jedenfalls dort ist mir aufgefallen, es steht nichts drin zur Zusammenarbeit von, mit der Betreuung. Umgekehrt haben wir aber in unserem Betreuungskonzept drin sehr wohl diese Schnittstellen und Austausch und Vernetzungen, wo wir versuchen auch strukturell zu verankern.“ (Schulhaus B, sozialpädagogische Fachkraft 1, 118).

Gemäss der Wahrnehmung dieser sozialpädagogischen Fachkraft, die in der Arbeitsgruppe zum Entwurf des Tagesschulkonzeptes mitgewirkt hat, wurde seitens der Lehrkräfte die Zusammenarbeit mit sozialpädagogischen Fachkräften nicht aufgeführt, während bei den sozialpädagogischen Fachkräften die Zusammenarbeit mit den Lehrkräften Bestandteil des Betreuungskonzepts ist. Gleichzeitig sind die strukturell gleichbehandelnden Bedingungen wie beispielsweise Teilnahme an Konferenzen und Arbeitsgruppen (Austauschge- 
fässe) oder Leitungsstruktur (Schulleitung und Leitung sozialpädagogischer Bereich) gegeben.

\section{Fazit}

Die Stadt Zürich führt als erste Schweizer Gemeinde Tagesschulen schrittweise und flächendeckend ein. Seit 2016 haben fünf bestehende Regelschulen den Tagesschulbetrieb aufgenommen, wobei einzelne Umstellungen, wie beispielsweise Austauschgefässe zwischen Lehrkräften und sozialpädagogische Fachkräften bereits im Vorjahr gestartet haben. Vor diesem Hintergrund setzte sich auch ein Prozess in der Zusammenarbeit zwischen Lehrkräften und sozialpädagogischen Fachkräften in Gang. Die im Beitrag vorgestellten Forschungsbefunde aus dem AusTEr-Projekt liefern zentrale Elemente für die Gestaltung und Wahrnehmung der Zusammenarbeit beider Professionen.

Ausgehend vom Professionsverständnis nach Nittel (2002) und aus der Datenauswertung lässt sich zusammenfassen, dass sich in den gedeuteten Handlungen von Lehrkräften und sozialpädagogischen Fachkräften die Zusammenarbeit im Sinne eines „Nebeneinanders“ und eines Koordinierens der beiden Arbeitsfelder abzeichnet. Mit einer Schule als Ausnahme zeigt sich keine inhaltliche Verzahnung der Arbeitsfelder.

In der Einführungsphase der Tagesschule beschränkt sich zudem die Zusammenarbeit zwischen Lehrkräften und sozialpädagogischen Fachkräften auf organisationale Absprachen und das Lösen von Problemfällen, umfasst hingegen kaum eine integrierte (sozial-)pädagogische Förderung der einzelnen Kinder. Die fachlichen Ressourcen sind noch ungenügend verzahnt und insbesondere das fachliche Potential der sozialpädagogischen Fachkräfte fliesst noch nicht optimal in gemeinsame Aktivitäten, wie beispielsweise Schulausflüge oder in thematische Arbeitsgruppen, ein.

Die Unkenntnis des Arbeitsfeldes der jeweils anderen Profession und die asymmetrischen sowie einseitigen Formen der Zusammenarbeit sind zwei zentrale Deutungsmuster aus der Datenauswertung, die der normativ gesetzten Erwartung nach Zusammenarbeit zwischen Lehrkräften und sozialpädagogischen Fachkräften entgegenstehen. Hier liegt ein klares strukturelles und personelles Entwicklungspotential im Einführungsprozess von Tagesschulen, wie beispielsweise Hospitationen (vgl. Flitner, 2017) und Visualisierung der fachlichen Angebote der anderen Profession.

Parallel zu den erwähnten Gelingensfaktoren aus anderen Studien gehen aus der Datenanalyse ähnliche Tendenzen hervor: Trotz teilweise vorhandener Austauschgefässe könnten der Informationsfluss sowie die zeitlichen und örtlichen Rahmenbedingungen für Zusammenarbeit im Zuge des Einfüh- 
rungsprozesses von Tagesschulen sowohl auf struktureller als auch auf individueller Ebene weiter optimiert werden.

\section{Literatur}

AusTEr (2016). AusTEr - Aushandlungsprozesse der pädagogischen Zuständigkeiten an Tagesschulen im Spannungsfeld öffentlicher Erziehung. Vom Schweizerischen Nationalfonds gefördertes Forschungsprojekt, verfügbar unter www.zhaw.ch/sozialearbeit/auster.

AvenirSocial (2011). Beschäftigung und Ausbildung im Bereich der Sozialen Arbeit in der Schweiz. Bestandsaufnahme und Perspektiven. Bern: AvenirSocial.

Bildungsdirektion Kanton Zürich (Hg.) (2017). Die Tagesschule - von der Idee bis zur Einführung. Zürich: Bildungsdirektion Kanton Zürich. Online verfügbar unter https://digitalcollection.zhaw.ch/handle/11475/1241, zuletzt geprüft am 21.03. 2017.

Böhm-Kasper, O., Dizinger, V. \& Heitmann, V. (2013). Interprofessionelle Kooperation an offenen und gebundenen Ganztagsgrundschulen. Zeitschrift für Grundschulforschung $\mathrm{H} 2$, 53-68.

Bohnsack, R. (1999). Gruppendiskussionsverfahren. Rekonstruktive Sozialforschung (S. 123-142). VS Verlag für Sozialwissenschaften.

Breuer, A. \& Reh, S. (2010). Zwei ungleiche Professionen? Wie LehrerInnen und ErzieherInnen in Teams zusammenarbeiten. Soziale Passagen 2 (1), 29-46.

Chiapparini, E., Schuler Braunschweig, P. \& Kappler, C. (2016). Pädagogische Zuständigkeiten in Tagesschulen. Diskurs Kindheits- und Jugendforschung, 11 (3), 355-361.

Chiocchio, F. \& Richer M. C. (2015). From Multi-professional to Trans-professional Healthcare Teams: The Critical Role of Innovation Projects. In Soyez, K. \& Gurtner, S. (Hg.), Challenges and Opportunities in Health Care Management. Cham: Springer, 161-169.

Coelen, T. \& Stecher, L. (Eds.) (2014). Die Ganztagsschule: Eine Einführung. Grundlagentexte Pädagogik. Weinheim: Beltz Juventa.

Dizinger, V. (2015). Professionelle und interprofessionelle Kooperation von Lehrerinnen und Lehrern im Kontext schulischer Belastung und Beanspruchung. Dissertation zur Erlangung des Doktorgrades der Philosophie (Dr. Phil.). Universität Bielefeld.

Fischer, N., Klieme, E., Holtappels, H.-G., Stecher, L. \& Rauschenbach, T. (2013). Ganztagsschule 2012/2013. Deskriptive Befunde einer bundesweiten Befragung. StEG 2012 - Ergebnisse der Schulleitungsbefragung. Frankfurt a.M., Dortmund, Gießen \& München.

Flitner, C. (2017). Sozialpädagogischer Auftrag der schulischen Tagesschulbetreuung. vpod bildungspolitik 222, 24-25.

Holtappels, H.-G., Lossen, K., Spillebeen, L. \& Tillmann, Katja (2011). Schulentwicklung und Lehrerkooperation in Ganztagsschulen. Konzeption und Entwicklungsprozess als förderliche Faktoren der Kooperationsentwicklung? In Fischer, N., 
Holtappels, H.-G., Klieme, E., Rauschenbach, T., Stecher, L. \& Züchner, I. (Hg.), Ganztagsschule: Entwicklung, Qualität, Wirkungen. Längsschnittliche Befunde der Studie zur Entwicklung von Ganztagsschulen (StEG). Weinheim: Juventa, 2542.

Kamski, I. (2011). Innerschulische Kooperation in der Ganztagsschule. Eine Analyse der Zusammenarbeit von zwei Berufsgruppen am Beispiel von Lehrkräften und Erzieherinnen und Erziehern. Münster, New York, NY, München, Berlin: Waxmann (Internationale Hochschulschriften, 556).

Merten, U. \& Kaegi, U. (Hg.) (2015). Kooperation kompakt. Professionelle Kooperation als Strukturmerkmal und Handlungsprinzip der Sozialen Arbeit. 1. Aufl. Leverkusen: Budrich, Barbara.

Nittel, D. (2002). Professionalität ohne Profession? „Gekonnte Beruflichkeit“ in der Erwachsenenbildung im Medium narrativer Interviews mit Zeitzeugen. In M. Kraul, W. Marotzki, \& C. Schweppe (Eds.), Biografie und Profession (S. 253286). Bad Heilbrunn: Verlag Julius Klinkhardt.

Nittel, D. (2011). Von der Profession zur sozialen Welt pädagogisch Tätiger? Vorarbeiten zu einer komparativ angelegten Empirie pädagogischer Arbeiten. In Helsper, W. \& Tippelt, R. (Hg.), Pädagogische Professionalität (Zeitschrift für Pädagogik, Beiheft: 57). Weinheim, 40-59.

Olk, T., Speck, K. \& Stimpel, T. (2011). Professionelle Kooperation unterschiedlicher Berufskulturen an Ganztagesschulen - Zentrale Befunde eines qualitativen Forschungsprojektes. In Stecher, L., Krüger, H.-H. \& Rauschenbach, T. (Hg.), Ganztagsschule - Neue Schule? Eine Forschungsbilanz, Sonderheft 15 der Zeitschrift für Erziehungswissenschaft. Wiesbaden, 63-80.

Paseka, A. (2011). Pfade der Professionalisierung - Roads (not) Taken. Journal für LehrerInnenbildung, 2, 5-10.

Santen, E. van \& Seckinger, M. (2003). Kooperation: Mythos und Realität einer Praxis. Eine empirische Studie zur interinstitutionellen Zusammenarbeit am Beispiel der Kinder- und Jugendhilfe. München: Dt. Jugendinstitut.

Schüpbach, M., Jutzi, M. \& Thomann, K. (2012). Expertise zur Kooperation in verschiedenen Kooperationsfeldern. Eine qualitative Studie zu den Bedingungen gelingender multiprofessioneller Kooperationen in zehn Tagesschulen. Zuhanden des Erziehungsdepartements. Bern: Universität Bern, Institut für Erziehungswissenschaft.

Schütze, F. (1983). Biographieforschung und narratives Interview. Neue Praxis, 13(3), 283-293.

Speck, K., Olk, T. \& Stimpel, T. (2011). Auf dem Weg zu multiprofessionellen Organisationen? Die Kooperation von Sozialpädagogen und Lehrkräften im schulischen Ganztag. Empirische Befunde aus der Ganztagsforschung und dem Forschungsprojekt „Professionelle Kooperation von unterschiedlichen Berufskulturen an Ganztagsschulen“ (ProKoop). In W. Helsper \& R. Tippelt (Hg.), Pädagogische Professionalität. Weinheim und Basel: Beltz Juventa (Zeitschrift für Pädagogik, Beiheft 57), 184-201.

Speck, K., Olk, T., Böhm-Kasper, O., Stolz, H.-J. \& Wiezorek, C. (Hg.) (2011). Ganztagsschulische Kooperation und Professionsentwicklung. Studien zu multiprofessionellen Teams und sozialräumlicher Vernetzung. Weinheim: Juventa. 
Stadt Zürich, Schul- und Sportdepartement (2017). Tagesschule 2025. Online verfügbar unter:

https://www.stadt-zuerich.ch/ssd/de/index/volksschule/tagesschule2025.html, zuletzt geprüft am 02.03.2018.

Strauss, A. \& Corbin, J. M. (1997). Grounded theory in practice. Sage.

Terhart, E. (1997). Berufskultur und professionelles Handeln bei Lehrern. In Combe, A. \& Helsper, W. (Hg.), Pädagogische Professionalität. Untersuchungen zum Typus pädagogischen Handelns. Frankfurt a.M.: Suhrkamp Taschenbuch, 448471. 


\title{
Fachliches Handeln an der Schnittstelle von Schule und Kindesschutz: Empirische Erkenntnisse zum Einsatz von Gefährdungsmeldungen in der Schweiz
}

\author{
Andreas Jud, Madlaina Stauffer \& David Lätsch
}

\section{Einleitung}

Die Schule ist ein zentraler Ort des Aufwachsens von Kindern und Jugendlichen - schon rein zeitlich kommt ihr nach der Familie die grösste Bedeutung zu. Lehrpersonen haben teils fast täglichen Kontakt mit denselben Schülerinnen und Schülern und können so ungünstige Entwicklungen von Kindern potentiell frühzeitig wahrnehmen. Die negativen Veränderungen können mit schulinternen Mitteln, durch Interventionen schulnaher Dienste wie Schulsozialarbeit und Schulpsychologie niederschwellig angegangen werden. Die Schulsozialarbeit ist vor allem dann relevant, wenn die Schwierigkeiten des Kindes nicht primär oder nicht ausschliesslich den Leistungsbereich betreffen und soziale Probleme wie Konflikte in Familien oder unter Gleichaltrigen angegangen werden müssen. Da der Standort von Schulsozialarbeit in der Schweiz häufig auf dem Areal der Schule ist oder die Schulsozialarbeit zumindest zeitweise zu fixen Sprechstunden vor Ort anwesend ist (z.B. Iseli \& Stohler, 2012, S. 176, 180-184), sind die Wege sowohl für betroffene Schülerinnen und Schüler als auch für besorgte Lehrpersonen kurz, was den Zugang vereinfacht. ${ }^{1}$ Die Interventionsmöglichkeiten der Schulsozialarbeit basieren in bedeutendem Mass auf Freiwilligkeit der Zusammenarbeit (z.B. Heeg \& Baier, 2013, S. 174f.), staatliche Zwangsmittel sind nicht vorhanden. Auch das dürfte, die Kontaktaufnahme betreffend, zu einer niedrigen Hemmschwelle beitragen.

Um einem Kind in der Schweiz den nötigen Schutz vor vernachlässigendem Verhalten und vor psychischer, physischer und sexueller Gewalt zu gewähren, kann es jedoch mitunter auch für Schulen und schulnahe Dienste entscheidend sein, rechtzeitig die verantwortlichen Organe des zivilrechtli-

1 Zahlen zur Verbreitung der Schulsozialarbeit in der Schweiz finden sich z.B. bei Baier (2015) oder Seiterle (2014). 
chen Kindesschutzes, also die Kindesschutzbehörde ${ }^{2}$, einzuschalten. ${ }^{3}$ Diese Handlung wird in der juristischen Sekundärliteratur ${ }^{4}$ gemeinhin als Gefährdungsmeldung bezeichnet (z.B. Fassbind, 2016). Eine Entscheidung von Fachpersonen im schulischen Kontext für oder gegen eine Gefährdungsmeldung ist mit Unsicherheit behaftet, denn zumeist fehlen wichtige Informationen. Es kann nicht lediglich auf eine bereits erfolgte Schädigung reagiert werden. Eine Prognose ist notwendig, wie die Entwicklung des betroffenen Kindes und seiner Familie mit oder ohne Meldung und Einbezug der Kindesschutzbehörde verläuft. Durch diese Entscheidung unter Unsicherheit sind Fachpersonen im schulischen Kontext einem Dilemma ausgesetzt, das in gleicher oder ähnlicher Form alle Fachpersonen trifft, die eine potentielle Gefährdung des Kindeswohls abwenden wollen (vgl. Goldstein, Freud \& Solnit, 1973): Fachpersonen können einem gefährdeten Kind den notwendigen Schutz verwehren, weil sie zu spät reagieren oder eine Meldung unterlassen. Andererseits besteht ebenso die Gefahr, dass sie ungehörigerweise die Privatsphäre der Familie beinträchtigen, weil sie bereits bei milden Problemen eine Meldung auslösen. Bislang fehlten im deutschsprachigen Raum jedoch Studien, welche Daten zu auslösenden oder hindernden Faktoren für eine Gefährdungsmeldung aus dem schulischen Kontext analysieren. Die vorliegende Arbeit beschreibt die Ergebnisse zweier Schweizer Studien, die sich erstmals dieser Forschungslücke widmen.

\section{Methodisches Vorgehen}

\section{Studie zur Rolle der Lehrpersonen}

Eine Studie der Berner Fachhochschule ging der Frage nach, welche Rolle Lehrpersonen im Kindesschutz einnehmen. Verbunden damit war einerseits die Absicht, die von den Lehrpersonen wahrgenommenen Aufgaben sichtbar $\mathrm{zu}$ machen. Andererseits ging es indes auch um die Identifikation von Lücken, um mögliche Aufgaben im Bereich des Kindesschutzes also, die von den Lehrpersonen nicht oder nur unzureichend wahrgenommen werden - und damit womöglich auf ein Funktionsfeld der Schulsozialarbeit verweisen. $\mathrm{Zu}$ diesem Zweck wurden Lehrpersonen zu ihrem Rollenverständnis, Kenntnis-

2 Da die meisten Kindesschutzbehörden gleichzeitig auch als Erwachsenenschutzbehörde walten, hat sich in der deutschsprachigen Schweiz die Abkürzung KESB für Kindes- und Erwachsenenschutzbehörde etabliert.

3 Vertiefende Literatur zum System des Kindesschutzes in der Schweiz und zu dessen gesetzlichen Grundlagen findet sich z.B. bei Rosch und Hauri (2016).

4 Der Begriff Gefährdungsmeldung taucht jedoch in den entsprechenden Gesetzestexten selbst nicht auf. 
stand und professionellen Handeln im Bereich des Kindesschutzes befragt. Weiter wurde untersucht, aus welchen Gründen sich Lehrpersonen dafür oder dagegen entscheiden, bei einer vermuteten Kindeswohlgefährdung eine Gefährdungsmeldung einzureichen oder in Zusammenarbeit mit anderen Fachpersonen der Schule auf eine solche hinzuwirken. Zur Stichprobenauswahl wurde unter den öffentlichen Schulorganisationseinheiten im deutschsprachigen Kanton Bern eine nach dem Urbanitätsgrad der Schulgemeinde geschichtete Zufallsstichprobe von 252 Schulleiterinnen und Schulleitern gezogen. Die Schulleitenden wurden mit der Bitte angeschrieben, den Link zur OnlineBefragung an ihre Lehrpersonen weiterzuleiten. An der Befragung beteiligten sich schliesslich 156 Lehrpersonen der Primar- und Sekundarstufe des Kantons Bern (vgl. Weber, 2015).

\section{Studie zu Gefährdungsmeldungen durch Schulen und schulische Dienste}

Für die Deutschschweiz wurde die Schnittstelle zwischen Schulen und dem zivilrechtlichen Kindesschutz erstmals ausführlich in einer vom Schweizerischen Nationalfonds geförderten Studie der Hochschule Luzern - Soziale Arbeit untersucht (Jud, 2012; Jud \& Gartenhauser, 2015). ${ }^{5}$ Im Fokus des 2013 abgeschlossenen Projekts „Gefährdungsmeldungen durch Schulen und schulische Dienste" stand das Handeln von Fachkräften in Schulleitung, Schulsozialarbeit und Schulpsychologie und der rechtliche Kontext für ihr Handeln (Rosch, 2012). Ziel war es, über die Erfassung laufender Fälle und eine Umfrage bei den genannten Fachkräften im schulischen Bereich zu prüfen, wie Merkmale des betroffenen Kindes und seiner Familie, aber auch Eigenschaften der Fachkräfte und ihrer Organisation mit der Entscheidung für oder gegen eine Gefährdungsmeldung zusammenhängen. Gleichzeitig analysierte die Studie in einer qualitativen Forschungsmethodik, wie die Entscheidung für eine Gefährdungsmeldung entsteht - vom ersten Gedanken daran bis hin zur tatsächlichen Entscheidung. Die Besprechung zentraler Erkenntnisse in Kapitel 3 dieses Artikels fokussiert auf Ergebnisse aus diesem qualitativ ausgerichteten Studienteil, die bisher noch nicht publiziert wurden. Eine detaillierte Besprechung von Ergebnissen der anderen Studienteile sowie eine Vertiefung der methodischen Zugänge findet sich an anderer Stelle (Jud, 2012; Jud \& Gartenhauser, 2015; Rosch, 2012).

Zur Analyse von Strategien bei (potentiellen) Gefährdungsmeldungen durch Schulleitungen, Schulsozialarbeit und Schulpsychologie wurden bis Frühling 2011 insgesamt 24 Leitfaden-Interviews, je acht für die drei genannten Berufsgruppen geführt. Die Auswertung der transkribierten Text-Daten

5 Der Erstautor dankt an dieser Stelle Regula Gartenhauser und Katinka Gomez-Bugari für die Datenerfassung und Beteiligung an der Auswertung. 
erfolgte gestützt auf die Qualitative Inhaltsanalyse nach Mayring (2007; Mayring \& Gläser-Zikuda, 2005).

\section{Ergebnisse der beiden Studien}

\section{Die Rolle der Lehrpersonen}

In der Studie zur Rolle der Lehrpersonen im Kindesschutz wird deutlich, dass die überwiegende Mehrheit der befragten Lehrerinnen und Lehrer die eigene Profession als einen wichtigen Akteur im Handlungsfeld betrachtet: Rund drei Viertel (76\%) sehen sich als zentralen Partner der Kindesschutzbehörden. Die Überzeugung, im Bereich des Kindesschutzes über genügend Wissen zu verfügen, hält mit der wahrgenommenen Bedeutung dieses Bereichs jedoch nicht Schritt. Nur etwas mehr als ein Drittel gibt an, über solides Wissen zu verfügen. Eine naheliegende Ursache dafür findet sich in der Ausbildung der Lehrpersonen: 93\% finden, dass die Themen Kindeswohl und Kindeswohlgefährdung hier nicht ausreichend behandelt wurden. Die Wissensdefizite spiegeln sich schliesslich auch in den selbstberichteten Gründen wider, aus denen Lehrpersonen auf eine Gefährdungsmeldung trotz Verdacht auf Kindswohlgefährdung verzichten. Am häufigsten führt Unsicherheit (45\%) zur Unterlassung einer Gefährdungsmeldung, etwa Unsicherheit bezüglich der eigenen Rechte und Pflichten im Rahmen des Kindesschutzes (22\%) oder der eigenen Beobachtungen (24\%). Weiter trägt die Angst vor negativen Folgen für das betroffene Kind (35\%) oder seine Familie (16\%) vergleichsweise häufig zu einem Verzicht bei. Nicht selten genannt wird schliesslich auch eine zeitliche Überlastung (12\%). Diese selbstberichteten Gründe lassen sich danach beurteilen, ob sie potenziell legitim (wie die Angst vor negativen Folgen für das betroffene Kind) oder eindeutig problematisch sind (wie eine vorhandene Unsicherheit über eigene Rechte und Pflichten). Bestimmt man nun diejenigen Kontextbedingungen, die eine solche problematische Entscheidung wahrscheinlicher machen, so ragen zwei Faktoren heraus. Erstens fehlen Lehrpersonen, die aus problematischen Gründen auf eine Gefährdungsmeldung verzichten, gemäss eigenen Angaben häufiger die zeitlichen Ressourcen, um im Alltag überhaupt auf mögliche Gefährdungssituationen ihrer Schulkinder einzugehen. Zweitens berichten diese Lehrpersonen signifikant häufiger, dass die Thematisierung einer möglichen Gefährdung von Schulkindern sie emotional stark belaste.

In der Interpretation lassen sich diese Befunde so verstehen, dass Lehrpersonen ihre Unsicherheiten bezüglich der eigenen Pflichten im Kindesschutz dann nicht aktiv angehen, wenn sie entweder keine zeitlichen Ressourcen wahrnehmen, die ihnen für diese Aufgabe zur Verfügung stünden, 
oder wenn sie befürchten, durch das aktive Thematisieren der Gefährdung emotional verstrickt zu werden. Allgemeiner verweisen die Ergebnisse der Studie darauf, dass Lehrpersonen einen Beitrag im Bereich des Kindesschutzes in der Regel leisten wollen, praktisch jedoch oft nicht über die Zeit und auch nicht über die erforderlichen Kenntnisse des Kindesschutzes verfügen. Diese Befunde stammen aus dem Kanton Bern und lassen sich insofern nicht ohne weiteres auf andere Kantone mit unterschiedlichen Systemen der Schule und Lehrerausbildung übertragen. Dennoch unterstreichen sie die Wichtigkeit der Frage, ob und ggf. inwiefern Schulsozialarbeitende die mangelnden Ressourcen der Lehrkräfte im Kindesschutz auf unterschiedlichen Ebenen kompensieren können und sollen.

\section{Gefährdungsmeldungen durch Schulleitung, Schulsozialarbeit und Schulpsychologie}

Fokus elterliche Kooperation

Besonders deutlich tritt in der Studie der Hochschule Luzern die elterliche Kooperationsbereitschaft oder ihr Fehlen als massgebender Faktor bei Gefährdungsmeldungen hervor. Die vorhandene Kooperationsbereitschaft der Eltern führt bei allen untersuchten Berufsgruppen - also Schulleitungen, Schulsozialarbeit und Schulpsychologie - dazu, dass kaum Gefährdungsmeldungen ausgelöst werden. Umgekehrt ist das Fehlen oder Ausbleiben der Kooperationsbereitschaft der entscheidende Faktor, eine Gefährdungsmeldung einzuleiten. Diese Fokussierung auf die elterliche Kooperation ist nachvollziehbar und gleichzeitig mit verschiedenen Tücken behaftet. Fachkräfte aus Schulleitungen, Schulsozialarbeit und Schulpsychologie sind auf die Mitarbeit der Eltern angewiesen, um bei Problemsituationen von Kindern erfolgreich Unterstützung auf freiwilliger Basis anbieten zu können. Gehen die Eltern nicht auf Vereinbarungen oder Unterstützungsangebote ein oder reagieren gar mit Widerstand, sind Hilfen meist auch dann nicht erfolgversprechend, wenn sie dem Bedarf des Kindes entsprechen. Nicht nur der schulische Kontext, auch die staatlichen Kindesschutzmassnahmen stützen sich grösstenteils auf ein Mindestmass an elterlicher Kooperation: So nennt Art. 308 Abs. 1 ZGB für die mit Abstand am häufigsten errichtete zivilrechtliche Kindesschutzmassnahme der Beistandschaft (vgl. Konferenz für Kindes- und Erwachsenenschutz (KOKES), 2016) als zentrale Funktion, dass Beistandspersonen «die Eltern in ihrer Sorge um das Kind mit Rat und Tat» unterstützen.

Die elterliche Kooperation stellt einen massgeblichen Faktor für gelingende Hilfe bei kindlichen Problemsituationen dar. Dass alle untersuchten Berufsgruppen in der Studie die Entscheidung für oder gegen eine Gefähr- 
dungsmeldung jedoch vorwiegend auf diesen Aspekt abstützen, lässt dennoch eine kindzentrierte Perspektive vermissen. Im Vordergrund sollte bei allem Handeln im Interesse des Kindes dessen Befindlichkeit und potentielle Beeinträchtigungen der weiteren Entwicklung stehen. Eltern kann es an Fertigkeiten mangeln, die notwendig sind, um ein Unterstützungsangebot gelingen zu lassen, selbst wenn sie durchwegs in allen Situationen kooperativ handeln und sich einsichtig zeigen. ${ }^{6}$ Beim Fokus auf elterliche Kooperation ist die Gefahr gross, nicht genügend zwischen Kooperationsbereitschaft und kooperativem Handeln zu unterscheiden. «Echt» kooperatives Handeln erwächst aus der Motivation, an den vorhandenen Problemen zu arbeiten und Veränderung zu erreichen. Neben dieser Veränderungsmotivation muss bei Klientinnen und Klienten institutioneller Hilfen mitunter auch lediglich von einer Kontaktmotivation ausgegangen werden (vgl. Miller \& Rollnick, 2002). Durch das Wahrnehmen der Kontakte mit der Institution und ihren Fachkräften wird nach aussen Kooperation signalisiert, um zu verhindern, dass weitergreifende Massnahmen eingeleitet werden. Entscheidend ist daher zu prüfen, ob sich nach kurzfristigen Bemühungen bisherige Verhaltensmuster wieder einschleichen oder sich tatsächlich bedeutsame Veränderungen im Befinden oder in der Entwicklung des Kindes ergeben.

Für Fachkräfte, die auf freiwilliger Basis mit Kindern, Jugendlichen und ihren Familien arbeiten - also auch die Schulsozialarbeit - ist die Identifikation fehlender Veränderungsbereitschaft stets auch mit der Frage verknüpft, ob noch Unterstützung und Hilfe im Rahmen der eigenen Möglichkeiten geboten werden kann oder eine andere, externe Fachperson oder Stelle eingeschaltet wird, die fortan die Führung im Fall übernehmen wird. Entsprechend besteht die Gefahr, dass eine Gefährdungsmeldung besonders auch von Schulsozialarbeitenden als Scheitern erlebt wird, da das Problem nicht niederschwellig im Einvernehmen aller Beteiligten gelöst werden konnte. Diese Assoziation mit eigenem Scheitern wird in den geführten Leitfaden-Interviews an mehreren Stellen deutlich, wenn bspw. explizit Gefährdungsmeldungen mit Verlieren gleichgesetzt werden oder davon berichtet wird, dass eine Gefährdungsmeldung abgewendet werden konnte, so als wäre nicht die Kindeswohlgefährdung das Übel, das es abzuwenden gilt, sondern die Gefährdungsmeldung selbst. Eine Schulsozialarbeiterin formuliert bspw. explizit «... da konnten wir die Gefährdungsmeldung nochmals abwenden» (I13, 2:68). Stellvertretend für alle untersuchten Berufsgruppen illustriert die Bezeichnung der Gefährdungsmeldung als «ultima ratio» in einem transkribier-

6 Auch Massnahmen zur Abwendung von Kindeswohlgefährdungen setzen keine Verschuldensabhängigkeit der Eltern voraus (vgl. Rosch \& Hauri, 2016). 
ten Ausschnitt ${ }^{7}$ aus einem Interview mit einem Schulleiter, dass grosse Widerstände gegen das Instrument der Gefährdungsmeldung vorhanden sind:

Für mich ischs würklich ultima ratio, ä Gföhrdigsmeldig, äs isch ä massive Iigriff id Famili [...] und möglichscht lang probierä, d'Lüüt is Boot z'hole odr im Boot z'bhalte, immer wieder probierä mit Gspröch, zeigä, dass mer do isch, uf ne diskreti Art probiert z'understütze, und wänn das eifach, ... grundsätzlich nümmä goht, nümmä möglich isch, dass mer si, diä Lüüt nüm erreicht, denn möched mer dä Schritt. [...] würd lieber warte und de i im letschtä Moment, aber no rächtzitig, da isch wichtig." (I21 (SL), 11:70, 143-145). ${ }^{8}$

Das Zitat weist auch auf die wiederholt auftretenden, latent widersprüchlichen Haltungen gegenüber Gefährdungsmeldungen hin, die sich ebenfalls deutlich bei allen untersuchten Berufsgruppen zeigen. Es stellt sich nämlich die Frage, ob die erwähnte Gefährdungsmeldung im letzten Moment überhaupt noch rechtzeitig sein kann. Wird somit nicht erst dann weitergegeben, wenn immer wieder ein neuer eigener Lösungsversuch gestartet wurde, obschon bereits mehrere vorherige Lösungsversuche nicht zielführend waren?

Jenseits elterlicher Kooperation

Weiter wird aus den Interviews auch deutlich, dass problembelastete Schülerinnen und Schüler oft bereits seit dem Kindergarten bekannt sind. Die verschiedenen Stufenwechsel sind nicht nur mit Wechseln bei den Lehrpersonen, sondern oft auch mit Wechseln bei den zuständigen Fachkräften in schulischen Diensten verknüpft. Die neu zuständigen Personen versuchen, die Probleme erneut niederschwellig anzugehen. Dabei nutzen sie Mittel, die bereits von ihren Vorgängerinnen und Vorgängern angewendet wurden, ohne dass sich die Problemsituation des Kindes deutlich verändert hätte. Zwar wird eine Gefährdungsmeldung daher oft bereits früh angedacht, steht dann aber über längere Zeit im Raum, ohne tatsächlich ausgelöst zu werden. Dieses längere Zögern in der Entscheidung für eine Gefährdungsmeldung trotz ausbleibender Verbesserung in der Situation des Kindes wird wiederholt damit begründet, einen Vertrauensverlust verhindern zu wollen. Auch wird befürchtet, durch die Gefährdungsmeldung den subsidiären Hilfeprozess ganz abzuklemmen. Einige Aussagen zum Zuwarten bei der Entscheidung für eine

7 Im Interesse der Leserlichkeit wurden Räuspern und andere Lautäusserungen nicht zitiert.

8 Sinngemässe Übersetzung in deutsche Schriftsprache: „Für mich ist es eine „ultima ratio“, die Gefährdungsmeldung. Sie ist ein massiver Eingriff in die Familie [...] und möglichst lange versuchen, die Leute ins Boot zu holen oder dort zu halten. Immer wieder im Gespräch aufzuzeigen versuchen, dass man hier ist, auf diskrete Weise versucht zu unterstützen, und wenn das einfach ... grundsätzlich nicht mehr geht, nicht mehr möglich ist, dass man die Leute erreicht, dann machen wir den Schritt [...] würde lieber warten und dann im letzten Moment, aber noch rechtzeitig - das ist wichtig. 
Gefährdungsmeldung haben auch den Charakter nachträglicher Rationalisierung des Zuwartens, etwa, dass man die Behörden nicht mit «Bagatellfällen» belasten wolle, damit genügend Kapazität für die «richtigen» Fälle vorhanden bleibe. Ein Schulsozialarbeiter fasst den Prozess des Hinausschiebens einer angedachten Gefährdungsmeldung wie folgt zusammen ${ }^{9}$ :

„[H]üüfig passierts eso, dass es wiene lineare Prozäss isch. Öpper macht mau, aso d'Lehrperson macht mau öppis, bis sie nüm cha. $U$ de chunt vilich no so d'IF, d'Heilpädagogin chunt när vilich dri, u de übernähme die relativ viu, [...] u nächär mache die o bis sie nüm chöi, u de chöme vilich mir dri oder e Fachsteu dri u so vergeit ganz viu Ziit, so isch d'Früeerfassig i däm Sinn eh ziitlich nüm gwährleischted, [...] u de chunt äbä die Gwöhnig dri““(I18 (SSA), 13:48, 50). ${ }^{10}$

Weiter werden strukturelle und administrative Hürden als hinderliche Faktoren für die Auslösung einer Gefährdungsmeldung identifiziert. So wird der mit einer Gefährdungsmeldung verbundene Aufwand als eher gross eingeschätzt. Viele der damaligen als Laienbehörden ausgestalteten Kindesschutzorgane wurden zudem als inkompetent und nicht geeignet für eine angemessene Bearbeitung einer Gefährdungsmeldung wahrgenommen. Als besonders hinderlich wird auch beurteilt, dass in Folge einer Gefährdungsmeldung keine Rückmeldungen mehr über den weiteren Verlauf des Falls erfolgen. Zwar ist eine solche aufgrund des Datenschutzes nur eingeschränkt möglich, nichtsdestotrotz könnte selbst eine kurze Nachricht, dass im Fall weitere Unterstützung angeboten oder der Fall zumindest bearbeitet wird, das Vertrauen der Melderin oder des Melders stärken.

Schliesslich wird auf die Gefahr hingewiesen, dass sich das Zuwarten nicht verselbstständigen dürfe und Fachpersonen stets mögliche Veränderungen der kindlichen Situation im Auge behalten sollten. Wann ist nun - abgesehen vom Aspekt elterlicher Kooperation - für die betroffenen Fachkräfte der Zeitpunkt gekommen, doch eine Gefährdungsmeldung auszulösen? Einige Interviewte geben an, sich am Leidensdruck des Kindes zu orientieren. Der Zeitpunkt der Entscheidung wird damit unbewusst auf das betroffene Kind übertragen, was insofern problematisch ist, da der Leidensdruck auch von erfahrenen Professionellen nicht immer adäquat erfasst werden kann und eine deutlich ungünstige Entwicklung für das Kind nicht immer mit einem ausgeprägten Leidensdruck verbunden sein muss. Die Schwelle für eine Ge-

9 Im Interesse der Lesbarkeit wurde das Zitat gekürzt.

10 Sinngemässe Übersetzung in deutsche Schriftsprache: „Häufig ergibt das sowas wie einen linearen Prozess. Jemand, z.B. die Lehrperson, macht mal was, bis nichts mehr geht und dann kommt vielleicht noch die IF, d.h. die Heilpädagogin, die macht dann wieder ziemlich viel [...] dann machen die wieder, bis sie nicht mehr können und dann kommen wir noch zum Zug oder eine Fachstelle und so vergeht viel Zeit, so ist die Früherfassung im eigentlichen Sinn nicht mehr gewährleistet [...] und dann kommt noch der Gewöhnungseffekt.“ 
fährdungsmeldung ist für die befragten Fachkräfte auch bei besonders schwerer Kindeswohlgefährdung überschritten, ohne dass sie jedoch genau benennen, was eine besonders schwere Kindeswohlgefährdung ist. Es wird meist lediglich davon gesprochen, dass die Gefährdung akut, gewichtig, massiv oder eben gross sein müsse. Explizit als Auslöser werden unmittelbare Gefahren für «Leib und Leben» genannt: Dazu gehören Suizidgefahr, aber auch sexuelle oder körperliche Übergriffe auf die Kinder und Jugendlichen. Vernachlässigendes Verhalten, obschon häufiger und in seinen Folgen keineswegs per se weniger schädlich für die Betroffenen als körperliche und sexuelle Gewalt, wird weniger häufig als Anlass für eine Gefährdungsmeldung genommen. Dabei dürfte auch massgeblich sein, dass Unterlassungen schwerer zu definieren und zuzuordnen sind als klarer verortbare aktive Einwirkungen.

\section{Fazit}

Die beiden besprochenen Studien weisen auf verschiedene Faktoren hin, die für die untersuchten Berufsgruppen im schulischen Kontext - Lehrpersonen, Schulsozialarbeit, Schulpsychologie und Schulleitung - bedeutsam für die Erwägung einer Gefährdungsmeldung waren. Während bei Lehrpersonen vor allem fehlende Wissens- und Zeitressourcen bedeutsam waren, zeigten sich bei der Schulsozialarbeit, Schulpsychologie und den Schulleitungen vermehrt Merkmale des Falls (Kooperation der Eltern, Angst vor Vertrauensverlust) oder der Zusammenarbeit mit der Behörde (administrativer Aufwand, Zweifel an Fachlichkeit) als relevant. Trotz diesen Unterschieden wird als Gemeinsamkeit deutlich, dass Bedarf nach einer verstärkt kindzentrierten Perspektive bei der Einschätzung von Kindeswohlgefährdung durch Fachkräfte im schulischen Kontext besteht. Elterliche Kooperation oder Bedenken gegenüber der Fachlichkeit der Kindesschutzbehörden sind zwar Erwägungen, die berechtigterweise in den Prozess der Entscheidung für oder gegen eine Gefährdung einfliessen können. Im Zentrum sollte jedoch die kindliche Befindlichkeit und Entwicklung stehen und die Frage, ob sich bei niederschwelligen Lösungsansätzen hier auch tatsächlich bedeutsame Veränderungen ergeben.

Besonders auch in der Schulsozialarbeit betonen Fachkräfte eine ziemlich strikte Trennung von freiwilligem schulischen Kontext auf der einen Seite und Zwangsmassnahmen im zivilrechtlichen Kindesschutz auf der anderen Seite. In dieser Einfachheit lässt sich die Trennung allerdings nicht aufrechterhalten. Das Ausmass an Freiwilligkeit resp. Zwang im Kontakt mit institutionellen Settings ist vielmehr graduell zu betrachten. Eine Gefährdungsmeldung kann stets als mögliche eskalierende Stufe bei «freiwilligen» Hilfen 
vorgesehen werden oder zumindest mitschwingen und damit den Raum der Freiwilligkeit bei der Zusammenarbeit einschränken. Mitunter wird die Möglichkeit einer Gefährdungsmeldung auch explizit als (verklausulierte) Drohung vorgebracht. In der Theorie zur Sozialen Arbeit wird die Gleichzeitigkeit von Hilfe und Kontrolle ausführlich unter dem Begriff des Doppelmandats besprochen (z.B. Staub-Bernasconi, 2017). Wurde in Präsentationen und Artikeln zu Studienergebnissen auf den Aspekt der Kontrolle hingewiesen, hat das bei einigen Fachkräften der Schulsozialarbeit Widerstand ausgelöst und möglicherweise das Selbstbild einer primär stützenden Institution verletzt. Eine stets kritische Reflexion der Rolle des eigenen Berufsstandes ist daher auch im Berufsalltag unumgänglich.

Eigene emotionale Unsicherheit wiederum ist bei der Einschätzung von Kindeswohlgefährdung nicht komplett vermeidbar, darf aber nicht der bestimmende Faktor werden. Die identifizierten Lücken rufen nach einer verstärkten Förderung kinderschutzspezifischer Weiterbildung im schulischen Kontext, die sowohl Wissensaspekte - etwa die Meldepflicht von Personen in «amtlicher Tätigkeit» nach Art. 443 Abs. 2 ZGB (vgl. Rosch, 2012) - aufgreift als auch den Umgang mit eigener Fachlichkeit bei Entscheidungen unter Unsicherheit thematisiert. Der Ruf nach verstärkter Weiterbildung wird durch Angaben zur Häufigkeit von Weiterbildung in der Luzerner Studie gestärkt: Bei Schulsozialarbeitenden, die in ihrer Berufskarriere sicherlich mit Kindeswohlgefährdung konfrontiert werden, hatte nur jede zweite Fachkraft eine Weiterbildung zum Kindesschutz angegeben. Die anderen untersuchten Berufsgruppen weisen gar noch schlechtere Anteile in Weiterbildungen im Kindesschutz auf. Die weiterbildenden Hochschulen sind wiederum in der Pflicht, verstärkt empirische Erkenntnisse in die eigenen Weiterbildungsangebote rückfliessen zu lassen.

\section{Referenzen}

Baier, F. (2015). Schulsozialarbeit in der Schweiz. In T. Olk \& K. Speck (Hrsg.), Reader Schulsozialarbeit - Band 3 (S. 41-61). Berlin: Deutsches Rotes Kreuz.

Fassbind, P. (2016). Verfahren vor der KESB: Von der Gefährdungsmeldung bis zur Vollstreckung. In D. Rosch, C. Fountoulakis \& C. Heck (Hrsg.), Handbuch Kindes- und Erwachsenenschutz: Recht und Methodik für Fachleute (S. 99-123). Bern: Haupt.

Goldstein, J., Freud, A. \& Solnit, A. J. (1973). Beyond the best interests of the child. New York: Free Press.

Heeg, R. \& Baier, F. (2013). Wirkungschronologien in der Schulsozialarbeit. In E. M. Piller \& S. Schnurr (Hrsg.), Kinder- und Jugendhilfe in der Schweiz (S. 165196). Wiesbaden: Springer VS. 
Iseli, D. \& Stohler R. (2012). Schulsozialarbeit aus der Perspektive des Sozialmanagements - Ergebnisse einer Modellanalyse in verschiedenen Kantonen. In H. Bassarak \& A. Schneider (Hrsg.), Forschung und Entwicklung im Management sozialer Organisationen (S. 173-190). Regensburg: Walhalla.

Jud, A. (2012). Zwischen Schule und Kindesschutz: Zur Rolle der Schulsozialarbeit bei Gefährdungsmeldungen durch Schulen. Sozial Aktuell, 44(4), 30-31.

Jud, A. \& Gartenhauser, R. (2015). The impact of socio-economic status and caregiver cooperation on school professionals' reports to child protection services in Switzerland. European Journal of Social Work, 18(3), 340-353. doi: 10.1080/13691457.2014.933093

Konferenz für Kindes- und Erwachsenenschutz (KOKES) (2016). KOKES-Statistik 2015: Anzahl Personen mit Schutzmassnahmen. Zeitschrift für Kindes- und Erwachsenenschutz, 71(4), 313-315.

Mayring, P. (2007). Qualitative Inhaltsanalyse: Grundlagen und Techniken (9 Aufl.). Weinheim: Deutscher Studienverlag.

Mayring, P. \& Gläser-Zikuda, M. (2005). Die Praxis der qualitativen Inhaltsanalyse. Weinheim: Beltz.

Miller, W. R. \& Rollnick, S. (2002). Motivational interviewing preparing people for change (2nd ed.). New York: Guilford Press.

Rosch, D. (2012). Melderechte, Melde- und Mitwirkungspflichten, Amtshilfe: Die Zusammenarbeit mit der neuen Kindes- und Erwachsenenschutzbehörde. Die Praxis des Familienrechts, 13(4), 1020-1051.

Rosch, D. \& Hauri, A. (2016). Zivilrechtlicher Kindesschutz. In D. Rosch, C. Fountoulakis \& C. Heck (Hrsg.), Handbuch Kindes- und Erwachsenenschutz (S. 410449). Bern: Haupt.

Seiterle, N. (2014). Schulsozialarbeit in der Deutschschweiz: Eine Standortbestimmung. In K. Gschwind (Hrsg.), Soziale Arbeit in der Schule: Definition und Standortbestimmung (S. 82-145). Luzern: interact.

Staub-Bernasconi, S. (2017). Soziale Arbeit und Menschenrechte: Vom beruflichen Doppelmandat zum professionellen Tripelmandat. Leverkusen: Budrich.

Weber, M. (2015). Lehrpersonen als Partner im Kindesschutz. Eine Standortbestimmung anhand des Kantons Bern und Empfehlungen für die Zusammenarbeit [Unveröffentl. Masterarbeit]. Bern: Berner Fachhochschule. 



\section{Perspektiven aus der französischen und deutschen Schweiz}





\title{
Positionierungen der Schulsozialarbeit in der französischsprachigen Schweiz
}

\author{
Hans-Peter von Aarburg und Jacques Kottelat
}

\section{Föderale Vielfalt}

Die Bildungslandschaft der Schweiz ist trotz breit abgestützter Harmonisierungsbemühungen föderal stark fragmentiert. Auch in den vier französischund den drei zweisprachigen Kantonen der Romandie arbeiten sonderpädagogische, schulpsychologische und sozialarbeiterische Dienste unter institutionell höchst unterschiedlichen Rahmenbedingungen.

Nicht nur unterscheiden sich institutionelle Gegebenheiten von einem welschen Kanton zum andern oft erheblich, auch der Kenntnisstand der im Bildungsbereich arbeitenden Fachpersonen über solche Gegebenheiten manchmal sogar anderer Kantonsteile - ist meist beschränkt. Nicht zuletzt auch die öffentliche Anerkennung der Schulsozialarbeit als einer Profession mit durchsetzbaren Standards leidet an einem dadurch behinderten fachlichen Austausch. Seit 2012 trifft sich deshalb eine Gruppe von französischsprachigen Schulsozialarbeitenden im Rahmen einer „Tour de Romandie“ einmal im Jahr, um sich fachlich auszutauschen und dabei auch berufspolitisch zu positionieren. Ihren fünften Halt machte die Tour im Mai 2017 an der Fachhochschule für Soziale Arbeit in Sierre. Zusammen mit „AvenirSocial“, einem gesamtschweizerischen Verband von Professionellen der Sozialen Arbeit, organisierten die beiden Autoren dieses Treffen. An Schulen sozialarbeiterisch engagierte Fachpersonen aus allen ganz oder teilweise französischsprachigen Kantonen nahmen daran teil. ${ }^{1}$ Es wurde dabei die grosse institutionelle Vielfalt sichtbar, die im Folgenden skizziert werden soll. Mit Ausnahme des Unterwallis arbeiten heute Fachpersonen der Sozialen Arbeit in all diesen Kantonen an öffentlichen Schulen.

Jacques Kottelat (2015, S. 66f.), Koordinator der unter dem Dach von AvenirSocial organisierten Gruppe von welschen Schulsozialarbeitenden, führt in einer nach Kantonen geordneten Tabelle insgesamt 155 Stellen auf, die in der Romandie der Schulsozialarbeit (SSA) zugeordnet werden können.

1 Kurz zusammenfassende Dokumente zu diesem jährlichen Treffen finden sich unter: http://www.avenirsocial.ch/f/f42001506.html [Zugriff: 04.01.2017]. 
Dabei werden allerdings Stellen mit höchst unterschiedlichen Mandaten, organisatorischen Anbindungen und unterschiedlichen Bezeichnungen erfasst, was Vergleiche schwierig macht.

Diese föderale Vielfalt soll hier im Rahmen einer konzeptionellen Fragestellung umrissen werden. Empirisch beruht die Darstellung hauptsächlich auf persönlichen Recherchen über fachliche Netzwerke und auf öffentlich zugänglichen Dokumenten, wie sie sich etwa auf Internetportalen von Schulen, Gemeinden, Kantonen und Berufsverbänden finden. Daneben konnte gelegentlich auch auf Bachelor- und Masterarbeiten zurückgegriffen werden. Insgesamt ist dieser Fachbereich wenig dokumentiert.

Der vorliegende Beitrag möchte in einem Überblick Entwicklungslinien aufzeigen, er hat keinen Anspruch auf Vollständigkeit.

\section{1 „Schulinterne“ oder ,schulexterne“ Mandate?}

Fachpersonen der Sozialen Arbeit übernehmen auch in der Romandie an vielen Schulen sozialpädagogische, sozialarbeiterische und animatorische Mandate, die früher meist an Lehrpersonen übertragen waren. Die dazu notwendige Kooperation fordert alle Beteiligten heraus, Mandate zu verhandeln und sich zu positionieren: Wie viel Erziehung und Sozialarbeit hat die Schule neben der Wissensvermittlung und der Selektion zu übernehmen? In welchem Auftrag handelt die Soziale Arbeit?

Für alle direkt betroffenen Fachpersonen (und ihre Hierarchien) scheint es dabei wichtig zu sein, wieweit und in welchem Sinn sie Teil der Schule sind. Im Folgenden brauchen wir deshalb das Begriffspaar „schulintern“ und „schulextern“, um professionelle Mandate, wie wir sie beobachten, in ihrer Nähe bzw. Distanz zur Schule darzustellen. Diese Unterscheidung drängt sich auf, wie etwa aus Drillings (2009, S. 66) Darstellung von Kooperationsformen herausgeht, die auf Wilfried Wulfers und Mechthild Seithe beruht. Diese räumliche Kategorie verwenden auch die gemeinsam vom Schweizer Schularbeitsverband (ssav) und von AvenirSocial herausgegebenen Leitlinien (2010, 2016) explizit im Begriffspaar „intégré“/,,additionné“ (,,integriert“/ „addiert"), auf die auch Kottelat (2015) zurückgreift.

Das Kriterium von ,innen“ und ,aussen“ wird hier nacheinander unter vier verschiedenen Gesichtspunkten gesehen. Als Erstes wird die (Nicht-)Zugehörigkeit zu einer Profession der Schulpädagogik verstanden, als Zweites die organisatorische Einordnung der mit Mandaten Beauftragten in eine schulische bzw. eine sozialarbeiterische Hierarchie, als Drittes die Ausprägung einer partnerschaftlichen Kooperation mit den Lehrpersonen und als Viertes die räumliche Organisation des Mandates als eines „ambulanten“ bzw. „,im 
Schulhaus integrierten“ Dienstes. Um ein flüssiges Lesen zu ermöglichen, werden die einzelnen Beispiele jeweils möglichst zusammenhängend geschildert, auch wenn sie streng genommen aufgespalten und stets unter jedem der vier Punkte besprochen werden müssten.

\subsection{Sozial-oder schulpädagogische Mandate?}

Schule und Jugendhilfe in Genf spannten schon in den 1960er Jahren zusammen, um gemeinsam ein niederschwelliges Beratungsangebot im schulischen Alltag anzubieten (Dupuis \& Oldacre, 2013). Der Kanton finanziert seither an allen Schulen der Sekundarstufe 1 Fachpersonen der Sozialen Arbeit, die hauptberuflich Jugendliche, aber auch deren Eltern und Lehrpersonen beraten und unterstützend begleiten. Kottelat (2015) beziffert die Zahl dieser als "Sozialberaterinnen und -berater " (,conseillers sociaux") bezeichneten Fachpersonen der Sozialen Arbeit mit 38. Rund ebenso viele Sozialberatende arbeiten auf der Sekundarstufe 2, also an verschiedenen Arten nachobligatorischer Ausbildungsgänge.

All diese Fachpersonen arbeiten in Schulgebäuden und sind ein integrierter Teil des pädagogisch tätigen Personals. Sie sind in einem eigenen Dienst der kantonalen Jugendhilfe organisiert. Über die Einzelfallarbeit hinaus wird von Sozialberaterinnen und -beratern insbesondere auch erwartet, dass sie sich für die Förderung eines vernetzenden Dialogs zwischen allen direkt oder indirekt im Schulbetrieb involvierten Akteurinnen und Akteuren einsetzen.

Als Sozialpädagoginnen und -pädagogen (,éducateurs sociaux“) werden Fachpersonen der Sozialen Arbeit an Genfer Primarschulen vom Kanton seit 2006 beschäftigt. Sie arbeiten an Schulen, die als sozial besonders belastet eingestuft werden und deswegen im Rahmen des Programms REP (,réseau d'enseignement prioritaire") zusätzliche kantonale Mittel erhalten (Ferri, 2015). Die Sozialpädagoginnen und -pädagogen sollen in enger Zusammenarbeit insbesondere mit Lehrpersonen, der Schulleitung, dem schulpsychologischen Dienst und andern Institutionen die Integration der Schülerinnen und Schüler in den schulischen Alltag fördern. Ein wichtiger Teil des Mandates ist es, die Schule in ihrer Zusammenarbeit mit den Eltern zu unterstützen.

Ausgehend von der Waadt hat sich an den Schulen der Romandie (ausser in Genf) ein Modell für niederschwellige sozialpädagogische Unterstützung entwickelt, welches in der Deutschschweiz unbekannt ist. Lehrpersonen mit einer Zusatzausbildung setzen sich hier als „schulische Mediatorinnen und Mediatoren“ für ein gutes Schulklima ein. Dazu unterstützen und beraten sie insbesondere Heranwachsende, zusätzlich aber auch Lehrpersonen und Eltern, vertraulich und auf freiwilliger Basis. „Schulische Mediation“ gehört in 
der ganzen Westschweiz (mit Ausnahme des Kantons Genf) zumindest an den Sekundarschulen meist schon seit Jahrzehnten zum niederschwelligen und vom regulären Schulbudget finanzierten Hilfsangebot. Das Zusatzmandat der Mediation wird den Lehrpersonen mit einer Unterrichtsentlastung abgegolten, im Kanton Fribourg etwa mit der Entlastung von einer Wochenlektion pro 120 Schülerinnen und Schüler, im Kanton Wallis mit einer solchen pro 250 Schülerinnen und Schüler. In einem Sammelband werden von Fachverantwortlichen aller Kantone der Romandie differenzierte Einblicke in kantonale Entwicklungen dieses Modells gegeben (Mirimanoff, 2013).

In den vielen Schulen der welschen Schweiz, wo Vertreterinnen und Vertreter der Schulpädagogik und der Sozialen Arbeit inzwischen alltäglich zusammenarbeiten, scheinen die spezifischen und komplementären Möglichkeiten der beiden Ansätze erkannt, die Aufgabenbereiche bei Überschneidungen aushandelbar zu sein. Im Kanton Fribourg etwa sind inzwischen „Schulmediation“ und „Schulsozialarbeit“ als komplementäre Angebote auf kantonaler Ebene geregelt. ${ }^{2}$

An allen Schulzentren des Kantons Waadt arbeiten vom Kanton finanzierte und organisierte Teams von Fachpersonen verschiedener Disziplinen, die sich um das physische, psychische und soziale Wohlergehen der Schülerinnen und Schüler bemühen. ${ }^{3} \mathrm{Zu}$ diesen Fachpersonen gehören neben Pflegefachleuten (,infirmières scolaires“) insbesondere auch die erwähnten „schulischen Mediatorinnen und Mediatoren“, also spezialisierte Fachpersonen der Schulpädagogik, bisher allerdings keine Fachpersonen der Sozialen Arbeit. Seit einem Wechsel an der Spitze des Erziehungsdepartementes scheinen mit dem Schuljahr 2017/2018 im Rahmen eines zweijährigen Pilotprojektes allerdings auch auf kantonaler Ebene Modelle aus dem Bereich der Sozialen Arbeit zum Zuge zu kommen, wie sie sich erfolgreich auf Gemeindeebene entwickelt haben ${ }^{4}$.

Wie verwirrend es sein kann, institutionelle Gegebenheiten über Sprachgrenzen hinweg zu vergleichen, zeigen etwa die von der Schweizerischen Konferenz der Erziehungsdirektoren zum Entwicklungsstand der „Schulsozialarbeit“ zusammengestellten Resultate einer Kurzbefragung kantonaler Dienststellen. ${ }^{5}$ Darin wird der Begriff „Schulsozialarbeit“ mit ,,service social scolaire“ übersetzt, worunter zwei professionelle Gruppen, die „conseillers

2 Reglement vom 19. April 2016 zum Gesetz über die obligatorische Schule (SchR), Artikel 19 https://bdlf.fr.ch/frontend/versions/4734?locale=de [Zugriff: 04.01.2018].

3 https:/www.vd.ch/themes/formation/sante-a-lecole/equipes-psps/ [Zugriff: 04.01.2018].

4 Communiqué de presse de l'Etat de Vaud du 15 août 2017

https://www.bicweb.vd.ch/frame.aspx?pPage=/communique.aspx?pObjectID=636652 [Zugriff: 04.01.2018]. http://www.edk.ch/dyn/16173.php [Zugriff : 26.08.2017]. 
sociaux“ (,Sozialberater") und die „,médiateurs scolaires“ (,schulischen Mediatoren"), als gleichwertig vorausgesetzt und erfasst werden.

\subsection{Organisatorisch der Schule oder der Jugendhilfe unterstellt?}

Wie in andern Kantonen gingen viele Initiativen, professionelle Jugendhilfe mit dem schulischen Alltag zu verbinden, auch im Kanton Waadt oft von Gemeinden (Meyer \& al., 2003) aus. Einzelne von diesen haben schon vor Jahrzehnten damit begonnen, Fachpersonen der Sozialen Arbeit damit zu betrauen, Schülerinnen und Schüler, Eltern und Lehrpersonen zu beraten und ihnen gegebenenfalls auch längerfristige, institutionell vernetzte Unterstützung anzubieten. Vielerorts werden diese Fachpersonen als „Beratende Schule-Elternhaus" (,conseillers école-famille“) bezeichnet. In der Stadt Lausanne, wo sie in einem eigenen, städtischen Sozialdienst organisiert sind, werden sie „Schulsozialassistentinnen/-assistenten“ (,assistantes sociales scolaires“) genannt. Die ersten von ihnen waren bereits in den frühen 1970er Jahren angestellt worden.

Gehen in der Waadt Initiativen für schulsozialarbeiterische Angebote offenbar eher von sozialen Diensten der Gemeinden als von Schulen aus, so scheinen sich entsprechende Angebote in den Kantonen Neuchâtel und Fribourg eher auf Anregung von Schulleitungen und in direkter Abhängigkeit von diesen zu entwickeln.

La Chaux-de-Fonds und Le Locle waren die ersten Gemeinden des Kantons Neuenburg, welche Fachpersonen der Sozialen Arbeit an ihren Schulen einstellten, dies ebenfalls schon in den 1970er Jahren. Inzwischen gibt es in allen sieben Schulkreisen des Kantons sozialpädagogische Dienste (,,services socio-éducatifs"). Je nach Schule werden diese an Primar- und Sekundarschulen tätigen Fachkräfte als "Sozialpädagogisch Beratende“" (,conseiller socio-éducatif") oder als „Schulassistentinnen und -assistenten“ (,assistants sociaux") bezeichnet. Kottelat (2015) zählt 25 Personen, die unter einem dieser beiden Titel im Kanton Neuenburg beschäftigt sind. Diese Fachpersonen werden von den Gemeinden bezahlt, sind dabei aber direkt den Direktionen von Primar- und Sekundarschulen unterstellt. Ihr Mandat besteht darin, Schulleitungen, Lehrpersonen, Eltern und Schülerinnen und Schüler sowohl in akuten Krisen als auch in einem eher präventiven Sinn zu unterstützen und zu beraten. Aurélie Brunner (2014) zeichnet in ihrer Masterarbeit die Entwicklung dieser Institution in diesem Kanton nach. Im Kanton Neuenburg werden an einzelnen Schulen inzwischen zusätzlich auch Sozialpädagoginnen und -pädagogen beschäftigt, die nicht als Teil des sozialpädagogischen Dienstes gelten. Das Mandat dieser Fachpersonen der Sozialen Arbeit beruht 
dabei weniger als anderswo auf dem Prinzip der Freiwilligkeit: Sozialpädagoginnen und -pädagogen sollen Schulleitungen und Lehrpersonen gezielt von alltäglichen erzieherischen Aufgaben entlasten, etwa indem sie sozial auffällige und störende Schülerinnen und Schüler betreuen, wenn diese im Rahmen einer erzieherischen Sanktion für eine Lektion vom Unterricht ausgeschlossen werden.

Auch an den Schulen mehrerer Freiburger Gemeinden, so an den Sekundarschulen ("Cycles d'orientation") von Romont und Estavayer, werden Schulsozialarbeitende (,travailleurs sociaux scolaires“) mit vergleichbaren Mandaten betraut. Diesen Fachpersonen, die einer Schulleitung unterstehen, ist es vorab aufgetragen, Schülerinnen und Schüler mit störendem Verhalten im Rahmen von erzieherischen Sanktionen zu betreuen. Ziel dabei ist es, problematische Entwicklungen möglichst frühzeitig mit einer massgeschneiderten Begleitung aufzufangen. Neben Einzelfall- und Gruppenarbeit sollen sich diese Fachpersonen auch in einem umfassenderen strukturpräventiven Sinn für ein gutes Schulklima einsetzen. Die Kosten für ihre Anstellung werden von Gemeinden und vom Kanton geteilt.

Die Schulsozialarbeit an den französischsprachigen Sekundarschulen der Stadt Freiburg hält unter ähnlichen institutionellen Rahmenbedingungen weit grössere Distanz zu den Schulleitungen und setzt stärker auf Unterstützung im Rahmen freiwilliger Beratung.

\subsection{Als aktive Partner in Schulteams eingebunden?}

Im ganzen Kanton Bern, auch im französischsprachigen Teil, gibt es institutionalisierte und gesetzlich geregelte Schulsozialarbeit, der Kanton beteiligt sich mit momentan 10 Prozent an ihren Kosten. So arbeiten in der zweisprachigen Stadt Biel inzwischen je vier deutsch- und französischsprachige Fachpersonen der Sozialen Arbeit. Ihre grosse Akzeptanz bei Lehrpersonen und Schulleitungen wurde erst kürzlich erneut bestätigt und hat zu einer deutlichen Erhöhung der Stellenprozente geführt. Die Sozialarbeitenden sind hier zuständig für alle Primar- und Sekundarschulen der Stadt, unterstellt sind sie einem städtischen Sozialdienst (Andreoli, 2016). Dabei sind sie - wie gewöhnlich auch andernorts - in schulische und ausserschulische Helfernetze eingebunden. Kottelat (2017) betont, wie wertvoll dieser interprofessionelle Austausch im Rahmen institutionalisierter regelmässiger Zusammenkünfte für den Schulbetrieb ist.

Nicht zuletzt wohl allseitig gute Erfahrungen mit der Bieler Schulsozialarbeit und auch ein kollegial engagierter Austausch zwischen Fachpersonen und Behörden dieser Stadt mit umliegenden französischsprachigen Gemein- 
den haben diese veranlasst, an ihren Primar- und Sekundarschulen ebenfalls Schulsozialarbeit einzuführen. Dies gilt für Moutier, Valbirse und Tramelan/Reconvilier. Weitere Gemeinden erwägen eine Einführung.

Schätzen Schulleitung und Lehrpersonen die Tätigkeit der hier eng in die Schule eingebundenen Fachpersonen der Sozialen Arbeit besonders deshalb, weil diese den Schulalltag mit einem im Vergleich zu den Lehrpersonen dezentriert schulexternen Blick sehen? Im Idealfall geben Lehrpersonen hier Erziehungsverantwortung nicht an Fachpersonen der Sozialen Arbeit ab, sondern teilen sie mit ihnen. Wenn über Einzelfälle hinaus eingespielte Verhaltensmuster reflexiv bearbeitet werden, bedeutet das letztlich Schulentwicklung. Eine solche kooperative Einbindung von Fachpersonen in Schulteams ist anspruchsvoll und wirft Fragen auf, die hier nur gestellt, nicht aber beantwortet werden können:

Wie weit können Fachpersonen der Sozialen Arbeit in ihrer Zusammenarbeit mit Schulen ihre professionelle Eigenständigkeit als aussenstehende Dritte bewahren? Wie weit gelingt es ihnen dabei, oft zu Konflikten führende Routinen im Schulalltag mit einem systemisch und sozialpolitisch geschärften Blick von aussen zu betrachten und auftretende Spannungen als Drittpersonen im Interesse aller Beteiligten zu entspannen? Können sie sich davor schützen, mit delegierten Hilfsfunktionen von der Schule vereinnahmt zu werden und dadurch ihre mediative Stellung zu verlieren?

\subsection{Niederschwellig in Schulgebäuden tätig?}

Neben sonderpädagogischen sind auch schulpsychologische Dienste in einzelnen Kantonen unterschiedlich eng in die Schulen eingebunden. In der Waadt etwa werden solche Dienste grundsätzlich in den Schulgebäuden angeboten und ermöglichen deshalb einen alltäglichen Austausch mit andern Akteuren des Schulbetriebs. Auch die hier im Auftrag von Gemeinden beschäftigten „Beraterinnen und Berater Schule Familie“ arbeiten meist in Schulgebäuden.

Im Gegensatz dazu befanden sich etwa im Kanton Neuenburg bis vor kurzem schulnahe Dienste, insbesondere die Schulpsychologie, räumlich meist ausserhalb von Schulgebäuden. Räumliche Distanz bzw. Nähe dürfte alltägliche Praktiken stark beeinflussen.

Da sich Schulsozialarbeit als niederschwelliges Angebot versteht, sollten die Fachpersonen für die beratenen Personengruppen über möglichst kurze Wege erreichbar sein. Nun sind aber insbesondere Primarschulen oft über mehrere Standorte verteilt, was eine ständige Präsenz der SSA in vielen lokalen Gegebenheiten verunmöglicht. Kompromisse mit festgelegten Präsenz- 
zeiten in kleineren Schulhäusern oder der Möglichkeit, bei Bedarf eine Fachperson auch kurzfristig herbeizurufen, sind deshalb nötig. Die räumlich verstandenen Kategorien von ,ambulanter“ und ,integrierter“ SSA (Iseli \& Grossenbacher-Wymann, 2013, S. 9) sollten deswegen in einem graduellen Sinn angewandt werden.

Im Kanton Jura etwa arbeitet gegenwärtig eine Fachperson der Sozialen Arbeit an den verschiedenen Primar- und Sekundarschulen der Stadt Delémont unterschiedlich intensiv ,,integriert“, eine andere Fachperson der Sozialen Arbeit steht allen andern Schulen im Kanton ,ambulant“ zu Verfügung.

\section{Der Prozess der professionellen Positionierung}

Wie in der Deutschschweiz scheint sich die SSA auch in der Romandie hauptsächlich ,von unten nach oben“ zu entwickeln. Ganz offensichtlich fördern die für die ganze Schweiz typischen föderalen Systeme vielfältige Formen der schulischen oder schulnahen Jugendhilfe auf Gemeindeebene, die sich erst allmählich in kantonalen und noch weiter gefassten Strukturen verfestigen. Die SSA der Westschweiz befindet sich in weiten Teilen noch in einer Pionierphase, in der einerseits vieles möglich ist, anderseits aber auch institutionelle Sicherheit und verlässliche Orientierungspunkte fehlen.

In der berufssoziologischen Tradition von Hughes und Abbot (2003) kann beobachtet werden, wie sich hier eine neue Berufsgruppe mit ihren Zielen und Methoden auf einem konkreten Feld gegenüber andern Akteuren behauptet. Diese Akteure sind Schulbehörden und Dienste der Jugendhilfe auf kantonaler und kommunaler Ebene, Schulleitungen, verschiedene Gruppen der Lehrerschaft. Sie gehen untereinander und mit Fachpersonen der Sozialen Arbeit Allianzen ein oder geraten in Rivalitäten. Dabei zeigen die Spannungsfelder je nach Kanton und zum Teil sogar nach Gemeinde oft ganz andere Muster und Eigendynamiken, die von aussen betrachtet zudem oft nur schwer nachvollziehbar sind.

Erstaunlicherweise beschäftigt sich in der ganzen Romandie momentan noch keine Fachhochschule eingehend mit der Thematik von Sozialer Arbeit an Schulen und stösst entsprechende Diskussionen an oder moderiert sie. Noch keine Fachhochschule bietet hier einen Weiterbildungskurs zu spezifischen Fragen in diesem Themenkreis an, etwa in Form eines CAS. Auseinandersetzungen mit dem Thema fanden bisher vor allem über Studienpraktiken und Bachelor- und Masterarbeiten statt.

Auch dass es in der Westschweiz einen Berufsverband für SSA wie erwähnt erst im Ansatz gibt, ist ein Hinweis darauf, dass die Pionierphase noch nicht abgeschlossen ist. Schulsozialarbeitende sind hier noch wenig überregi- 
onal organisiert, sie finden sich oft isoliert an ihren Arbeitsorten. Unter diesen Bedingungen berufliche Standards zu entwickeln und durchzusetzen, ist schwierig, zumal sich institutionelle Gegebenheiten wie erwähnt zumindest von Kanton zu Kanton meist stark unterscheiden und Modelle und Erfahrungen deshalb nicht ohne weiteres übertragbar sind. Ein überregionales berufspolitisches Engagement in der Freizeit bringt zwar nur selten einen unmittelbar sichtbaren Nutzen, ist aber unverzichtbar.

\section{Literatur}

Abbott, A. (2003). Ecologies liées. Apropos du système des professions. In P.-M. Menger (Ed.), Les professions et leurs sociologies (pp. 29-50). Paris: Editions de la Maison des sciences de l'homme.

Andreoli, L. (2016). Problématiques scolaires: un terrain de coopération pour les travailleurs sociaux et travailleuses sociales et les enseignant-e-s?. Sierre: Mémoire de bachelor: HETS.

AvenirSocial \& ssav. (2010). Lignes directrices: qualité dans le travail social en milieu scolaire.

AvenirSocial \& ssav. (2016). Leitbild Soziale Arbeit in der Schule - Lignes directrices du travail social en milieu scolaire. Luzern, Bern: https://ssav.ch/de/publikationen/grundlagenpapiere [Zugriff: 02.03.2018].

Brunner, A. (2014). Le travail social en milieu scolaire dans le canton de Neuchâtel. Travail de master HES-SO. Lausanne.

Drilling, M. (2009). Schulsozialarbeit. Antworten auf veränderte Lebenswelten (4. ed.). Bern: Haupt.

Dupuis, G. \& Oldacre, N. (2013). Etre travailleur social à l'école. La construction du rôle de conseiller social et ses enjeux dans un cycle d'orientation. Genève.

Ferri, A. (2015). Apporter un autre regard. En milieu scolaire primaire, la présence d'éducateurs est très récente. actualité sociale, 55(03), 9-10.

Iseli, D. \& Grossenbacher-Wymann, S. (2013). Travail social en milieu scolaire. Lignes directrices (3e édition ed.). Berne: DIP.

Kottelat, J. (2015). Tournée romande: les travailleurs sociaux sont de plus en plus nombreux en milieu scolaire. actualité sociale, 55(03), 7-9.

Kottelat, J. (2017). Le groupe social scolaire: une démarche qui tend à favoriser l'inclusion scolaire? In L. Gremion \& e. al. (Eds.), Vers une école inclusive. Regards croisés sur des défis actuels (pp. 39-54). Ottawa: Presses universitaires.

Meyer, V. et al. (2003). Schulsozialarbeit in der Romandie. In M. Drilling (Ed.), Schule und Soziale Arbeit. Aspekte interdisziplinärer Zusammenarbeit (S. 34-42). Basel: Verlag Fachhochschule für Soziale Arbeit beider Basel.

Mirimanoff, J. A. (Ed.) (2013). Médiation et jeunesse: mineurs et médiations familiales, scolaires et pénales en pays francophones. Bruxelles: Larcier. 


\title{
Ausserschulische Betreuung in der Romandie - Herausforderungen und Gedankenanstösse
}

\author{
Claudia Mühlebach und Marianne Zogmal
}

Die Autorinnen des Artikels sind Vorstandsmitglieder der Westschweizer Plattform für familienergänzende Kinderbetreuung (ProEnfance 2017) und im Bereich der ausserschulischen Betreuung (l'accueil parascolaire) tätig. Die folgenden Ausführungen basieren auf ihren beruflichen Erfahrungswerten. Angesichts des äusserst komplexen Arbeitsfeldes und der vielfältigen Gestaltungsformen der familienergänzenden Betreuung in der Schweiz geben die Autorinnen mit den ausgewählten Beispielen der Kantone Genf und Waadt einen Einblick in die aktuelle ausserschulische Betreuung in der französischen Schweiz. Insbesondere wird der Blick auf die Gestaltung der ausserschulischen Betreuung und - bezogen auf die Betreuungsqualität - auf den Auftrag und die Qualifikation des Personals mit Bezug auf die Betreuungsqualität gerichtet.

Die Politik ist bemüht und bestrebt, den Eltern die Möglichkeit zu geben, Beruf und Familie miteinander zu vereinbaren. Bei der Umsetzung der ausserschulischen Betreuung gibt es jedoch grosse Herausforderungen, insbesondere im Hinblick auf den genauen Auftrag, den entsprechenden gesetzlichen Rahmen und die Zusammenarbeit mit unterschiedlichen Beteiligten sowie die geografischen und finanziellen Rahmenbedingungen.

\section{Ausserschulische Angebote in Genf und Lausanne}

In den verschiedenen Kantonen der Romandie ist die ausserschulische Betreuung - ähnlich wie in der Deutschschweiz - unterschiedlich ausgestaltet (SODK 2015). Durch die Annahme des HarmoS-Konkordats (HarmoS 2008) der Kantone Waadt und Genf im April 2008 haben sich diese verpflichtet, eine ganztätige Bildungsmöglichkeit (journée continue) anzubieten. Allerdings sind die Kantone frei in der Entscheidung, inwiefern sie die ganztägigen Bildungsangebote, wie beispielsweise die ausserschulische Betreuung, anbieten 
und welche Umsetzungsmöglichkeiten sie ausarbeiten. Im vorliegenden Artikel sollen am Beispiel der Kantone Genf und Waadt zwei unterschiedliche Zugänge für die Umsetzung aufgezeigt werden. Während im Kanton Genf eine einheitliche und etablierte, gemeindeübergreifende ausserschulische Betreuungsform gewählt wurde, sieht dies im Kanton Waadt heterogener aus. Daher fallen die Ausführungen zum Kanton Genf knapper aus, während die ausserschulischen Angebote im Kanton Waadt in ihrer Vielfalt vorgestellt und diskutiert werden.

\section{Genf:}

Das erste kantonale Gesetz über die Schaffung von Horten und Mittagstischen (classes gardiennes et de réfectoires scolaires) wurde am 5. Juni 1886 erlassen (Panardelli, 2006, S. 27). In Genf existiert somit seit über 130 Jahren ein ausserschulisches Angebot. Heute werden die Bestimmungen zu den Tagesstrukturen im kantonalen Bildungsgesetz ausgeführt. Diese ausserschulischen Angebote fallen in den Verantwortlichkeitsbereich des Genfer Gemeindeverbands (association des communes genevoises), der wiederum einen entsprechenden Auftrag an die interkommunale Gruppe für ausserschulische Betreuung (groupement intercommunal pour l'animation parascolaire GIAP) erteilt hat (Art. 29 Abs. 1). Das ausserschulische Angebot muss für alle Kinder zwischen 4 und 12 Jahren verfügbar sein, die eine öffentliche Primarschule besuchen. Dabei haben die Schule und die ausserschulische Betreuung in Genf klar getrennte Rollen. Das ausserschulische Betreuungsangebot verfügt über separate Räumlichkeiten und Mitarbeitende ausserhalb des Schulareals. Zudem werden die Arbeitsleistungen nicht miteinander koordiniert, beziehungsweise nur infolge der persönlichen Initiative von Mitarbeitenden. Schliesslich untersteht das Schulsystem der Kantonshoheit, während die ausserschulische Betreuung auf Gemeindeebene geregelt wird.

Was die Beiträge der Eltern angeht, ist der Kanton Genf sehr sozial: Sie decken $20 \%$ des Budgets ab (Sekretariat des Grossen Rats Genf, 2011). 2016 beliefen sich die Kosten für die ausserschulische Betreuung auf 58 Millionen Franken (ebd.). In den letzten Jahren ist dieser Betrag aufgrund der wachsenden Nachfrage stetig gestiegen. 2013 nutzten 65,5\% der Schülerinnen und Schüler ein ausserschulisches Angebot (ebd.). Diese rasante und umfassende Entwicklung führt zu Engpässen: So müssen neue Mitarbeitende gefunden werden, um mit der steigenden Nachfrage Schritt zu halten. 1200 Fachkräfte sind derzeit in der Kinderbetreuung tätig. Jahr für Jahr müssen 200 neue Teilzeitangestellte eingestellt werden. 95\% der Angestellten sind weiblich, da das Einkommen als Nebenerwerb gilt (Arbeitspensen von $25 \%$ bis $50 \%$ ) und entsprechend für Alleinerziehende nicht ausreicht. Diese Situation führt zu einer hohen Personalfluktuation (ebd., S. 3). 
Das kantonale Gesetz sieht vor, dass das Angebot für alle Kinder zugänglich sein muss, definiert jedoch keine Mindestanforderungen bezüglich der Ausbildung der Mitarbeitenden (Status der ständigen Mitarbeitenden der GIAP, vgl. GIAP, 2017). Die interkommunale Gruppe verlangt eine Ausbildung mit eidgenössischem Fähigkeitszeugnis sowie Französischkenntnisse auf Stufe B2. Nach der Anstellung werden den Mitarbeitenden interne Weiterbildungen angeboten (120 Stunden obligatorische fachspezifische Weiterbildung). Zudem wird derzeit ein Katalog mit 70 Weiterbildungen für die Betreuenden ausgearbeitet. Die Absolventen erhalten jedoch kein Diplom. Diese Bedingungen sollen angesichts der Arbeitszeit realistisch sein und ermöglichen, die steigende Nachfrage nach ausserschulischen Betreuungsplätzen hinsichtlich der nötigen personellen Ressourcen zu bewältigen.

\section{Waadt:}

Mit dem Beitritt zu HarmoS (HarmoS 2008) entschied die Waadtländer Bevölkerung im November 2009 die Aufnahme von Artikel 63a in die kantonale Verfassung, gemäss dem die Gemeinden «während der ganzen obligatorischen Schulzeit ein für die Familien fakultatives ausserschulisches Betreuungsangebot in der Form einer Tagesschule» zu organisieren haben (Verfassung des Kantons Waadt, Art. 63a).

Diese Änderung wurde bereits 2009 verabschiedet, kommt jedoch erst per 1. Januar 2018 zur Anwendung (vgl. Grosser Rat des Kantons Waadt, 2017). Aktuell ist der Zugang zur ausserschulischen Betreuung im Kanton Waadt im Gegensatz zu Genf noch nicht für alle Kinder gewährleistet. Deshalb hat nun jede Gemeinde die HarmoS-Elemente wie etwa die ausserschulische Betreuung für Kinder in Form einer Tagesbetreuung selbständig umzusetzen. Konkret sind die Gemeinden aufgefordert, folgende Mindestangebote zur Verfügung zu stellen (revidiertes Gesetz zur Kindertagesbetreuung des Kantons Waadt, 2017):

- Betreuter Mittagstisch für Kinder von 4 bis 15 Jahren

- Betreuung nach Schulschluss für Kinder von 4 bis 12 Jahren

- Morgendliche Betreuung vor Schulbeginn für Kinder von 4 bis 10 Jahren

- Ein Betreuungsangebot am Mittwochnachmittag für Kinder von 4 bis 10 Jahren

Die freiwillige Nutzung des Angebots, welche die Gemeinden grundsätzlich zur Verfügung zu stellen haben, ermöglicht die Vereinbarkeit von Beruf und Familie. Da die Gemeinden für das Angebot zuständig sind, obliegt es diesen auch, die Leitlinien für die ausserschulische Betreuung festzulegen, was bisher in den Zuständigkeitsbereich der Fachstelle Tagesbetreuung des Kantons 
(office cantonal d'accueil de jour) fiel (OAJE, 2017). Um zu verhindern, dass jede Gemeinde eigene Prozesse einführt, hat der Gesetzgeber die Schaffung einer interkommunalen Einrichtung für die ausserschulische Betreuung an Primarschulen (établissement intercommunal pour l'accueil parascolaire primaire) beschlossen (EIAP, 2017). Diese Einrichtung, deren zehn Delegierte von den Gemeinden ernannt werden, ist nun für die Ausarbeitung eines Referenzrahmens für die ausserschulische Betreuung zuständig. Dieser soll sämtliche, für die Eröffnung eines Betreuungsangebots einzuhaltenden Voraussetzungen umfassen, insbesondere die Anzahl der Kinder pro Betreuungsperson, die Anzahl der Betreuungspersonen pro Kindergruppe, architektonische Voraussetzungen, die erforderliche Anzahl von Quadratmetern sowie alle weiteren relevanten Rahmenbedingungen. Danach wird die Aufsicht über den Referenzrahmen an die kantonale Fachstelle OAJE delegiert.

Das ausserschulische Betreuungsangebot, in Kollektiveinrichtungen oder in individueller Form, etwa durch Tagesfamilien, hat Anspruch auf Subventionen von der Waadtländer Stiftung für die Tagesbetreuung (fondation pour l'accueil de jour des enfants - FAJE). Finanziert wird diese öffentlich-private Partnerschaft durch einen Arbeitgeberbeitrag (2016 betrug dieser 0,08 \% der Lohnmasse im Kanton), einen Sockelbeitrag der Gemeinden (CHF 5.- pro Einwohner), einen Kantonsbeitrag (der je nach Lohnsumme des Betreuungspersonals in den Einrichtungen variiert) und eine Spende der Loterie Romande. Diese Beiträge (2017 insgesamt 73 Millionen) werden den Betreuungsangeboten in Form einer Subventionierung der Lohnsumme der Fachkräfte ausgezahlt (25\% im Jahr 2017, Jahresbericht der Stiftung für die Tagesbetreuung; FAJE, 2017, S. 11), sofern die Strukturen bestimmte Anforderungen erfüllen (insbesondere müssen sie Teil eines Betreuungsnetzwerks sein). Es steht den Gemeinden frei, sich einem Betreuungsnetzwerk anzuschliessen, um in den Genuss der Subventionen zu kommen, oder sich selbständig zu organisieren (ohne kantonale Subventionen).

Die Kinderbetreuung des Kantons Waadt trägt vier politischen Bereichen Rechnung: Sozialpolitik, Wirtschaftspolitik sowie Bildungs- und Gleichstellungspolitik. Aus diesem Grund hat der Gesetzgeber beschlossen, den Auftrag der Betreuungsangebote im Rahmen des neuen Gesetzes auszuführen (LAJE von 2006, 2017 revidiert). Die ausserschulischen Betreuungseinrichtungen müssen «die körperliche, emotionale und soziale Entwicklung der Kinder unter Berücksichtigung der Hauptverantwortlichkeit der Eltern und eines Rahmens gemäss einem pädagogischen Projekt fördern, das auf das Alter und die Bedürfnisse der Kinder zugeschnitten ist» (Gesetz zur Kindertagesbetreuung des Kantons Waadt 2017, S. 2). Da die Tagesbetreuung «die Chancengleichheit und die soziale Integration der Kinder» unterstützen soll (op.cit., Abs. b), ist das Gesetz zudem sozial und präventiv. 
Die interkommunale Einrichtung EIAP arbeitet derzeit an der Ausarbeitung des Referenzrahmens, der ab Januar 2018 gelten soll. Aktuell findet die Anhörung der verschiedenen Interessengruppen statt: Gewerkschaften, Betreuungsnetzwerke, Arbeitgeberverband, Stiftung für die Tagesbetreuung, Elternverein usw.

Die Tatsache, dass der Auftrag im Gesetz verankert wird, ist ein neuartiger und begrüssenswerter Ansatz. Trotz der Aufnahme ins Gesetz ist es aber nicht möglich, diese Aufgaben zu erfüllen, ohne Debatten zu führen und Überlegungen rund um das Kind und dessen Bedürfnisse anzustellen. Im folgenden Kapitel wird auf die Aufgaben eingegangen, um aufzuzeigen, wie wichtig es ist, diese ausführlich zu klären.

\section{Der Auftrag}

Im Auftrag der ausserschulischen Betreuungsangebote steht unumstritten das Kind im Mittelpunkt und damit zusammenhängend die Rolle der Betreuungspersonen. Dies ist am Beispiel der Kantone Genf und Waadt ersichtlich.

In Genf muss das ausserschulische Betreuungsangebot für alle Kinder zugänglich sein, was in den letzten Jahren zu einem deutlichen Anstieg der Anzahl der Betreuungsplätze geführt hat. Der Hauptauftrag in Genf besteht darin, die steigende Anzahl der Kinder zu bewältigen.

Im Kanton Waadt ist der Zugang zu den ausserschulischen Angeboten für viele Kinder erst ab 2018 möglich. Das LAJE sieht vor, «im gesamten Kantonsgebiet genügend Betreuungsplätze anzubieten, die für die Eltern finanziell tragbar sind und ihnen erlauben, Beruf und Familie zu vereinbaren» (LAJE, S. 2). Der Zugang zum Betreuungsangebot ist somit auch in Zukunft nur bedingt gewährleistet. Gemäss dem kantonalen Gesetz sind demnach der Zugang (unter gewissen Bedingungen) und der sozialpädagogische Auftrag sowie Ziele, die parallel umzusetzen sind, sicherzustellen. Da der Auftrag der ausserschulischen Betreuungsangebote auf Bundesebene nicht eindeutig definiert ist, können die Angebote unterschiedlichste Formen annehmen, wie beispielsweise Gruppenaktivitäten, Betreuungseinheiten für Schüler, Jugendzentren, Mittagstische oder beaufsichtigte Hausaufgaben. Das Angebot umfasst somit Aufsicht, Lernaktivitäten, Sozialisierung, Unterstützung, Betreuung, Mahlzeiten, Aktivitäten usw. Es erscheint den Autorinnen wichtig, Überlegungen zum Kind und zu dessen Bedürfnissen im Laufe des Tages anzustellen. Dabei sollte nicht unterschieden werden, ob sich das Kind bei seiner Familie, in der Schule oder in einer kollektiven Betreuungseinrichtung aufhält. Der Ansicht der Autorinnen nach ist es entscheidend, dass das Alter 
und die Bedürfnisse des Kindes berücksichtigt werden und eine Kontinuität in dessen Alltag geschaffen wird.

Vor mehreren Jahren hat der Vorstand von Bildung und Betreuung des schweizerischen Verbands für die schulische Tagesbetreuung einen Qualitätsrahmen für die schulische Tagesbetreuung (Bildung und Betreuung, 2017) veröffentlicht. Er geht dabei von einer schulischen Tagesbetreuung aus, welche:

- «die Rahmenbedingungen für erfolgreiches Lernen verbessert

- die Sozialkompetenz fördert

- aktive Integrationsarbeit leistet

- mehr Bildungsgerechtigkeit schafft

- zur Gesundheitsförderung und Gewaltprävention beiträgt

- die Eltern unterstützt

- die Vereinbarkeit von Beruf und Familie sowie die Gleichstellung von Mann und Frau ermöglicht»

(Bildung und Betreuung, 2017)

Die Betreuungsplätze sind sowohl in der Waadt als auch in Genf aus politischen Gründen in erster Linie für Kinder von Einelternfamilien gedacht, bei denen die Mutter oder der Vater einer Erwerbstätigkeit nachgeht, arbeitslos ist oder eine Aus- oder Weiterbildung absolviert, sowie für Familien, bei denen beide Elternteile arbeiten. Gewisse Eltern sehen im Angebot jedoch weit mehr als ein Mittel, ihre Kinder betreuen zu lassen: «In den Agglomerationen und den ländlichen Gemeinden wurde die Vereinbarung der Betreuungszeiten mit den Arbeitszeiten der Eltern als Hauptgrund genannt, während in den Städten die ausserschulische Betreuung oft aufgrund der informellen Lernmöglichkeiten gewählt wird. Diese wurden in der Romandie zudem häufiger als in der Deutschschweiz als Grund angegeben» (Stern \& Gschwend, 2015, S. 32).

Es wäre interessant, diese Aussage zu vertiefen und zu ermitteln, inwiefern die kollektiven Betreuungseinrichtungen Brücken schlagen zwischen dem Lernen in der Schule und dem von den Eltern als informell bezeichneten Lernen. Es wäre denkbar, dass die Betreuungseinrichtungen als Mittler zwischen Familien und Schule agieren, insbesondere, wenn Schwierigkeiten auftreten.

Da die Betreuenden einen sozialpädagogischen Auftrag erfüllen, ist die Thematik der Aus- und Weiterbildung der Mitarbeitenden im Zusammenhang mit der Zurverfügungstellung eines zufriedenstellenden Betreuungsangebots entscheidend. 


\section{Ausbildung vs. Betreuungsqualität}

Gemäss F. Pirard (2014) wird eine qualitative hochwertige Betreuung erreicht, indem "der Umgang mit den Kindern und den Familien, die Umsetzungsbedingungen und allgemein die betroffenen Politikbereiche hinterfragt werden, ohne dabei im Voraus einen Rahmen zu definieren ...» (Pirard, 2014, S. 13). Es ist eindeutig, dass das blosse Verfassen von Protokollen mit Verhaltensanweisungen für das Betreuungspersonal bei Weitem nicht ausreicht. Um auf die entsprechenden Bedürfnisse eingehen zu können, ist ein hohes Ausbildungsniveau erforderlich. Die Anforderungen im Themenbereich sind äusserst vielfältig und zahlreich, weshalb die folgende Aufzählung, die auf unserem Erfahrungswert basiert, nicht abschliessend ist: Sicherstellung der sozialen Integration, Unterstützung beim Lernen in der Schule oder in der Gesellschaft, Betreuung der Kinder bei schwierigen familiären, sozialen oder schulischen Veränderungen und Förderung der Eigenständigkeit. Die alltägliche Arbeit muss immer wieder neu hinterfragt werden, die umgesetzten Lösungen müssen in einen Kontext eingebunden werden und vertiefte Kenntnisse über die emotionale, kognitive und soziale Entwicklung der Kinder sind nach wie vor unerlässlich, um den Auftrag der ausserschulischen Betreuung zufriedenstellend wahrnehmen zu können.

Angesichts der erwähnten Aufträge herrscht in den Betreuungsangeboten ein akuter Mangel an qualifiziertem Personal. Der Anteil der Mitarbeitenden ohne Ausbildung ist mit bis zu $47 \%$ der Angestellten hoch (IWSB, 2016, S. 29). Ein Grossteil der Erwachsenen, die sich um die Kinder kümmern, verfügt nicht über eine entsprechende Ausbildung oder höchstens über eine minimale Grundausbildung. Unter diesen Bedingungen ist es nicht möglich, eine qualitativ hochwertige Betreuung zu gewährleisten.

Bei einem Betreuungsteam, das aus Fachkräften mit verschiedenem Ausbildungshintergrund und Mitarbeitenden ohne fachspezifische Ausbildung zusammengesetzt ist, stellen sich folgende drei offene Fragen:

- Welche Rolle nehmen die einzelnen Beteiligten in den Betreuungsangeboten ein?

- Welcher Mehrwert geht mit einer sozialpädagogischen Ausbildung einher?

- Welche Fachkompetenzen werden beim Erwerb und der Vermittlung der fachlichen Betreuung aufgebaut?

Gegenüber den Kindern und deren Familien sowie innerhalb des Teams steht der unterschiedliche Ausbildungshintergrund der Beteiligten in den ausserschulischen Angeboten oft nicht im Vordergrund. Zudem herrscht eine flache Arbeitshierarchie. Gerade die fehlende Differenzierung der unterschiedlichen 
Ausbildung des Personals in den ausserschulischen Angeboten erschwert es, den spezifischen Beitrag der einzelnen Personen zu ermitteln. Dennoch ist die weitere Professionalisierung sehr zentral. Wie Studien aufzeigen, sind beispielsweise die Fachkompetenzen im Bereich der Kinderbetreuung nicht „sichtbar" genug:

«Bei der Erziehung handelt es sich um eine alltägliche Tätigkeit. Zudem weist sie die Besonderheit auf, dass es dabei ,keine bestimmten Gesten' gibt, die eindeutig wahrgenommen und verstanden werden können. Beobachtet man eine erfahrene Erzieherin bei der praktischen Arbeit, ist ihr Know-how somit unsichtbar, da ihre Kompetenzen praktisch nicht erkennbar sind» (Coquoz, 2009, S. 11).

Die Berufe im Bereich der Kinderbetreuung werden vorwiegend von Frauen ausgeübt und erhalten nach wie vor nicht die verdiente Anerkennung, die erlauben würde, die geleistete Arbeit „zu erklären“. Aus einer soziologischen Perspektive besteht die Schwierigkeit, ",die Arbeit zu erklären', in einem sprachlichen Defizit, das unter anderem darauf zurückzuführen ist, dass die ,Begriffe zur Beschreibung der Aktivität 'von den dominanten sozialen Gruppen produziert und kontrolliert werden; diese sind nicht in diesem Bereich tätig, sondern organisieren die Arbeit oder entwickeln Materialien» (Boutet et al., 1976, S. 72).

Die Problematik der Kompetenzen der Berufe im Bereich der Kinderbetreuung ist komplex. Es handelt sich hauptsächlich um Interaktionskompetenzen (Zogmal et al., 2013). Diese ermöglichen es, sich in Situationen an die Kinder anzupassen, die deren Initiative oder Teilnahme am täglichen Leben fördern.

\section{Soziale Arbeit im schulischen und ausserschulischen Bereich}

Anfang der 2000er-Jahre schuf das Genfer Erziehungsdepartement (département de l'instruction publique) ein Prioritäres Lernnetzwerk (réseau d'enseignement prioritaire - REP) für die Primarschulen (Anzules, 2016). Dieses Projekt wurde für Schulen in Quartieren ausgearbeitet, die einen «hohen Anteil an fremdsprachigen Familien aufweisen, die unter prekären wirtschaftlichen und sozioprofessionellen Bedingungen leben, und in denen viele Kinder Probleme beim Lernen und Verhalten in der Schule haben» (op.cit, S. 183). Den betroffenen Schulen steht eine Sozialpädagogin oder ein Sozialpädagoge in Vollzeit zur Verfügung. Diese Fachperson soll eine Beziehung zwischen Schule und Familie sowie mit allen Akteuren aufbauen, die mit dem Kind in Kontakt stehen. Eine solche Sozialpädagogin oder ein solcher Sozialpädago- 
ge kann so auch Beziehungen zwischen dem Lehrpersonal und den Fachkräften im Bereich der ausserschulischen Betreuung knüpfen.

Wie wichtig der gesellschaftliche Zusammenhalt und die Betreuung von Kindern mit sozial herausforderndem Hintergrund (grosse soziale, familiäre oder schulische Problemen) sind, zeigt sich auch im Kanton Waadt. In diesem gibt es spezifische Betreuungsangebote für Schülerinnen und Schüler, die der Gefahr eines Schulabbruches entgegensehen, beispielsweise das MATAS-Programm. Dies ist ein Angebot der sozialpädagogischen Politik im Bereich des Jugendschutzes vom 28. August 2006 (Departement für Bildung und Jugend), in dessen Rahmen ausserschulische temporäre Aktivitäten angeboten werden, die eine zeitlich begrenzte vertragliche Zusammenarbeit zwischen der Schule und spezialisierten Institutionen darstellen. Ferner laufen im Kanton Waadt seit dem Schulbeginn 2017 ähnliche Pilotprojekte (Syndicat des Enseignants Romands (SER), 2017).

So sollen an den Schulen Stellen für Betreuende mit sozialpädagogischen Aufgaben geschaffen werden. Diese sind dabei hauptsächlich für die Beaufsichtigung und Betreuung der Schüler zuständig, die gemäss der Schule und den Eltern «verhaltensauffällig» sind (Maendly, 2017). Das Projekt, das in einer ersten Phase an sechs Schulen des Kantons Waadt zur Anwendung kommt, entstand insbesondere aufgrund der wiederholten Nachfrage der Schulen. Mehrere Schulen haben jedoch bereits eigene Massnahmen im Bereich der Sozialen Arbeit umgesetzt und nicht gewartet, bis der Kanton aktiv wurde (vgl. AvenirSocial 2016 und Zeitschrift ActualitéSociale (AvenirSocial, 2015)). Die Finanzierung erfolgte dabei in mehreren Fällen durch die Gemeinden - zulasten anderer Bereiche. In der Zusammenfassung des Berichts der Pädagogischen Gesellschaft des Kantons Waadt (société pédagogique vaudoise) mit dem Titel «educateurs dans les écoles» (Sozialpädagogische Fachkräfte an den Schulen) wird darauf verwiesen, wie wichtig solche Massnahmen sind. Das Dokument erwähnt aber auch die dabei auftretenden Schwierigkeiten bezüglich Organisation, Struktur und Finanzierung. Was die Ziele der sozialen Arbeit an den Schulen angeht, führt der Bericht auf, dass oft erst im letzten Moment darauf zurückgegriffen wird. Zudem «sind sich die befragten Personen einig darüber, dass die Arbeit der Betreuenden eine präventive Wirkung haben sollte. Es sollte nicht sein, dass diese erst im letzten Moment zum Einsatz kommen» (Prod'homme, 2014, S. 7).

Die Beispiele in den Kantonen Genf und Waadt zeigen, wie wichtig die sozialpädagogischen Fachkräfte und die Soziale Arbeit an den Schulen ist. Diese Arbeit, die auch von den Elternvereinen erwartet und begrüsst wird, hat jedoch zu weiterführenden Überlegungen zu führen, damit sie nicht nur in bestimmten herausfordernden Situationen und für Kinder mit besonderen so- 
zialen Bedürfnissen beigezogen wird, sondern für alle Kinder im Sinne einer präventiven Tätigkeit zugänglich wird.

\section{Fazit}

Die Autorinnen haben versucht, in der gegebenen Kürze die äusserst komplexe Situation der sozialpädagogischen Arbeit mit Kindern und deren Familien zu skizzieren. Bei den ausserschulischen Betreuungsangeboten für Heranwachsende gibt es grosse Herausforderungen bezüglich Chancengleichheit, Integration und Betreuung bei schwierigen Veränderungen im Umfeld des Kindes. Die Betreuungstätigkeit umfasst somit zahlreiche Facetten der Sozialen Arbeit. Es gilt, alle Beteiligten in den ausserschulischen Angeboten und die involvierten Institutionen in die Ausarbeitung von Lösungen miteinzubeziehen. Zudem können qualitativ hochwertige ausserschulische Betreuungsangebote nur sichergestellt werden, wenn die Mitarbeitenden über eine angemessene sozialpädagogische Ausbildung und ausreichende Qualifikationen verfügen.

Trotz der zahlreichen und unbestrittenen Bedürfnisse existiert bis heute kein klar definierter Arbeitsrahmen für die Umsetzung der ausserschulischen Angebote. Alle Beteiligten aus Politik und Schule, welche ein Konzept der ausserschulischen Angebote entwickeln, sind sich allerdings darin einig, dass sowohl die Heranwachsenden zu berücksichtigen sind, welche die ausserschulischen Betreuungsangebote nutzen, als auch solche, die dies noch nicht tun.

Allgemein kann festgestellt werden, dass weitere Überlegungen zu den Aufträgen der verschiedenen Akteure und Einrichtungen zweifellos erlauben würden, Klarheit bezüglich der pädagogischen und sozialen Massnahmen zu schaffen. Diese können jedoch nur angemessen angewandt werden, wenn sie von geschultem Personal umgesetzt werden.

\section{Literatur}

Anzules, D. (2016). Contrer l'échec scolaire en favorisant la participation de tous. In: B. Vittori (Dir.). Au risque de la prévention. Genf: Editions IES.

AvenirSocial (2015). ActualitéSociale ${ }^{\circ}{ }^{\circ} 55$. Verfügbar unter: http://www.avenirsocial.ch/de/p42013776.html [Zugriff: 02.03.2018].

AvenirSocial (2016). Leitbild Soziale Arbeit in der Schule. Verfügbar unter: www.avenirsocial.ch/cm_data/AS_DE_Schulsozarbeit_160329.pdf [Zugriff: 02.03. 2018].

Bildung und Betreuung (2017). Schweizerischer Verband für schulische Tagesbetreuung. Verfügbar unter: http://www.bildung-betreuung.ch [Zugriff: 02.03.2018]. 
Boutet, J., Fiala, P. \& Simonin-Grumbach, J. (1976). Sociolinguistique ou sociologie du langage? Critique, 344 (Januar), S. 68-85.Coquoz, J. (2009). Les défis de l'éducation précoce et les enjeux de la formation. Revue [petite] enfance, 101, S. $10-14$.

EIPA (2017). Verfügbar unter: https://www.bicweb.vd.ch/communique.aspx?pObjectID=532908 [Zugriff: 02.03 . 2018].

Fondation pour l'accueil de jour (2017). Jahresbericht der Fondation pour l'accueil de jour, Seite 11. Verfügbar unter: https://faje-vd.ch/parution-du-rapport-annuel-2016-5/ [Zugriff: 02.03.2018].

GIAP (2017). Groupement intercommunal pour l'animation parascolaire. Verfügbar unter: http://www.giap.ch/ [Zugriff: 02.03.2018].

IWSB (2016). Fachkräftesituation in den sozialen Berufen in drei Bereichen (Kinder, Menschen mit Beeinträchtigungen, Menschen im Alter). Basel: IWSB/Savoir Social.

Le Grand Conseil du Canton de Vaud (2017). LOI modifiant celle du 20 juin 2006 sur l'accueil de jour des enfants. Verfügbar unter:

http://proenfance.ch/images/actualites/vaud_laje_170131.pdf [Zugriff: 02.03.2018].

Maendly, Vincent (2017). Les élèves ingérables mettent l'école au défi. 24heures du 24.8.2017. Verfügbar unter:

https://www.24heures.ch/vaud-regions/eleves-ingerables-mettent-ecoledefi/story/29922645 [Zugriff: 02.03.2018].

OAJE (2017). Verfügbar unter: https://www.vd.ch/oaje [Zugriff: 02.03.2018].

Palandella, L. (2006). Vive le parascolaire! Entre l'école et la famille à Genève depuis 1886. GIAP. Genf: Tricorne Editions.

Pirard F. (2014). La qualité de l'accueil fait débat. Revue [petite] enfance, 113, S. 13-19.

Prod'homme N. (2014). Synthèse du rapport du GT SPV, «Educateur dans les écoles», Société pédagogique vaudoise.

ProEnfance (2017). Platforme romande pour l'accueil de l'enfance. Verfügbar unter: http://www.proenfance.ch/ [Zugriff: 02.03.2018].

Secrétariat du Grand Conseil de Genève (2011). Rapport de la Commission des pétitions chargée d'étudier la pétition du personnel de l'animation para scolaire (GIAP) relative à la politique d'austérité menée par le Conseil d'Etat. Verfügbar unter: http://ge.ch/grandconseil/data/texte/P01714A.pdf [Zugriff: 02.03.2018].

SODK (2015). Konferenz der kantonalen Sozialdirektorinnen und Sozialdirektoren. Verfügbar unter: http://sodk.ch/ [Zugriff: 12.03.2018]

Syndicat des Enseignants Romands (SER) (2017). Rentrée scolaire dans le canton de Vaud. La Société pédagogique vaudoise (SPV) communique. Verfügbar unter: http://www.le-ser.ch/actualites/rentr\%C3\%A9e-scolaire-dans-le-canton-de-vaudla-spv-communique [Zugriff: 02.03.2018].

Ville de Genève (2014). Les droits de l'enfant en ville de Genève; Rapport à l'intention du Conseil administratif. Verfügbar unter: http://www.ville-geneve.ch/fileadmin/public/Departement_5/Publications/ 2014/Rapport-droits-enfants-ville-Geneve.pdf [Zugriff: 02.03.2018]. 
Zogmal, M., Trébert, D. \& Filliettaz, L. (2013). En quoi les éducatrices et les éducateurs de l'enfance sont-ils compétents? Présentation d'une démarche de recherche. Revue [petite] enfance, 110, S. 79-89.

\section{Webseiten}

Leitbild Soziale Arbeit in der Schule (2016). Verfügbar unter: www.avenirsocial.ch/cm_data/AS_DE_Schulsozarbeit_160329.pdf http://www.proenfance.ch

Alle Informationen über die Revision des Gesetzes zur Kindertagesbetreuung des Kantons Waadt sind verfügbar unter:

http://www.rsv.vd.ch/rsvsite/rsv_site/doc.pdf?docId=5374\&Pvigueur=2018-08-

$01 \&$ Padoption $=2010-11-09 \&$ Pcurrent_version $=\&$ PetatDoc $=F \&$ Pversion $=$ $4 \&$ docType $=$ loi\&page_format $=\mathrm{A} 4 \_3 \&$ isRSV$=$ true $\&$ is $S J L=$ true \&outformat $=$ pdf \&isModifiante $=$ false

Interkantonale Vereinbarung über die Harmonisierung der obligatorischen Schule (HarmoS-Konkordat) (2007). Verfügbar unter:

https://edudoc.ch/record/24711/files/HarmoS_d.pdf

\section{Zeitschriften}

Actualité sociale, Ausgabe zum Thema soziale Arbeit in den Schulen (März 2015), point fort/Bien vivre à l'école 


\title{
Auf der Suche nach einem gemeinsamen Bildungsverständnis. Ein strukturtypisierender Orientierungsrahmen für die Zusammenarbeit von Schule und Kinder- und Jugendförderung
}

\author{
Anna Maria Riedi und Martin Biebricher
}

Mit einem Augenzwinkern meint Deinet (2015, S. 38), dass McDonald's die erfolgreichste Jugendeinrichtung in Deutschland ist. Was könnten Kinderund Jugendförderung sowie Schule von McDonald's lernen und wie können sie ihre jeweiligen Stärken in die Zusammenarbeit einbringen? An Beispielen aus der Forschungs- und Arbeitspraxis geht der Beitrag diesen Fragen nach und stellt einen strukturtypisierenden Orientierungsrahmen für die Zusammenarbeit von Kinder- und Jugendförderung und Schule zur Diskussion.

\section{Kinder- und Jugendförderung in der Schweiz}

Kinder- und Jugendarbeit wird zunehmend auch in der Schweiz als Kinderund Jugendförderung bezeichnet. Sie bietet Freizeitaktivitäten, die neben Spiel, Bewegung und Projektarbeit auch Raum für informelle Begegnungen, Feiern und Musse beinhalten. Zudem werden niederschwellige Beratungen für kinder- und jugendspezifische Fragen angeboten. Kinder- und Jugendförderung ermöglicht so pädagogische Freizeiträume. Diese sind zwischen Schonraum (Chiapparini, 2017) und Talentraum (Spierts, 1998) resp. selbstorganisierter und angeleiteter Förderung situiert. Sie sind freiwillig sowie partizipativ orientiert und gelten damit - im Gegensatz zu Angeboten von Schule, Sport- und Musikvereinen - als Freiräume für Selbstbildung und Selbstwirksamkeit. Im Kern geht es dezidiert um „Selbständigkeit, Anerkennung und Raum zur Entfaltung" (Steiner, 2015).

Zur Kinder- und Jugendförderung zählen im Kanton Zürich beispielsweise verbandliche, kirchliche und Offene Kinder- und Jugendarbeit (http://www. okaj.ch/ueber-uns/zielgruppen), im nationalen Kinder- und Jugendförderungsgesetz ist die Rede von ausserschulischer Arbeit, namentlich ,verbandliche und offene Arbeit mit Kindern und Jugendlichen samt niederschwelligen Angeboten“" (Art. 5 lit. a KJFG vom 30. September 2011, SR 446.1). Die Angebote ha- 
ben unterschiedliche Trägerschaften (Marti, 2011, S. 1) und Traditionen. In ländlichen Gebieten bestehen heute noch Jungmannschaften in der Nachfolge der Burschenschaften des 17. Jahrhunderts (für Europa vgl. Krummenacher 2014, S. 19). Sie pflegen die dörfliche Freundschaft, die Geselligkeit und sorgen für die Erhaltung alter Bräuche. So pflegt beispielsweise die Jungmannschaft Klosters im Kanton Graubünden das Brauchtum des „Chränza, Plumpna, Graba“ (https://www.jungmannschaft-klosters.ch/dr-verein/), was in etwa bedeutet: Festkränze, insbesondere Hochzeitskränze, anfertigen, mit Schellen oder Glocken an einem Umzug teilnehmen sowie der Besuch bei einem auswärtigen Liebhaber eines weiblichen Mitglieds der Jungmannschaft. In der Regel sind die Mitglieder unverheiratet - aber nur noch vereinzelt ausschliesslich männlich, wie beispielsweise bei der Knabengesellschaft Urdorf im Kanton Zürich (http://knabengesellschaft.ch/).

Gegen Ende des 19. und Anfang des 20. Jahrhunderts bildeten sich verschiedene überregionale Kinder- und Jugendvereinigungen resp. -verbände bspw. Pfadfinder- resp. Pfadfinderinnenbund, Cevi (Christlicher Verein Junger Männer/Frauen), Jungwacht/Blauring, Töchter- resp. Jünglingsbund des Blauen Kreuzes, Sozialistische Jugend/Rote Falken etc. (vgl. auch Krummenacher 2014, S. 21-27). Im Gegensatz zu den Jungmannschaften verfügen die Jugendverbände national und regional in der Regel über ehrenamtliche und professionelle Strukturen.

In den 1960er Jahren entsteht ergänzend dazu in der deutschsprachigen Schweiz die Offene Kinder- und Jugendarbeit, die keine Mitgliedschaft erfordert, in der Regel koedukativ ausgerichtet ist und sich zunächst vor allem im städtischen Raum im sogenannten Jugendhaus etabliert, beispielsweise das bereits 1963 eröffnete Juhu Winterthur, Kanton Zürich (vgl. Torresan, Cabral, Tobler \& Rommel, 2015, S. 8). Ab Mitte der 1980er Jahre haben sich die Angebote der Offenen Kinder- und Jugendarbeit zunehmend erweitert und ausdifferenziert (Gerodetti \& Schnurr, 2013, S. 829). In der französischund italienischsprachigen Schweiz bestehen vergleichbare Angebote in der Regel unter dem Label , animation socioculturelle' resp. , animazione socioculturale‘ (vgl. bspw. Schenker \& Wettstein, 2013, S. 46).

Anfang der 1990er Jahre etablieren sich in grösseren Gemeinden der deutschsprachigen Schweiz Fachstellen, die mit sogenannten Jugendbeauftragten die Vernetzung der lokalen Kinder- und Jugendangebote sicherstellen und die Kinder- und Jugendförderung strategisch weiterentwickeln (vgl. KKJF, 2010). Teilweise unterstehen ihnen auch Angebote der Schulsozialarbeit, bspw. Siggenthal im Kanton Aargau (Riedi \& Stohler, 2013). An anderen Orten bilden die Fachstellen zusammen mit der Schule und den Tagesstrukturen (Mittagstisch etc.) ein Geschäftsfeld der Gemeindeverwaltung, bspw. Egg im Kanton Zürich (https://www.egg.ch/verwaltung/abteilungen/bildung/). 
Mit Inkrafttreten des neuen KJFG im Jahre 2013, welches das bisherige Bundesgesetz über die Förderung der ausserschulischen Jugendarbeit (JFG) von 1989 ablöste, hat sich der Begriff der Kinder- und Jugendförderung anstelle der Jugendarbeit zunehmend etabliert.

\section{Kinder- und Jugendförderung wirkt}

Politisch weitgehend unbestritten ist der Nutzen der Kinder- und Jugendförderung, das zeigte die parlamentarische Debatte zum neuen Gesetz: „Damit leistet die ausserschulische Arbeit anerkanntermassen einen wichtigen Beitrag sowohl zur Entwicklung von Kindern und Jugendlichen zu Personen, die Verantwortung für sich selber und für die Gemeinschaft übernehmen, als auch zu ihrer sozialen, kulturellen und politischen Integration“ (Ständerat Theo Maissen, Kommissionssprecher, Amtl. Bull 2011, S 170). Hingegen zeigen sich spezifische Herausforderungen im empirischen Nachweis ihrer Wirkung. Befunde zur Wirkung werden in der Regel dann nachgefragt, wenn Legitimationen von Angeboten benötigt werden, meist im Verbund mit eingeschränkten finanziellen Ressourcen. Evaluationen sollen den Grad der Effizienz sowie die Wirksamkeit der Angebote aufzeigen, um nicht (noch mehr) finanzielle Einschränkungen hinnehmen zu müssen (vgl. Riedi \& Biebricher, 2013; Riedi \& Stohler, 2013). Dabei zeigt sich aber die Schwierigkeit, dass sich in biografischer Sicht Selbstbildung und Selbstwirksamkeit kausal nicht unmittelbar und ausschliesslich auf die Nutzung von Angeboten der Kinder- und Jugendförderung zurückführen lassen.

Dass Kinder- und Jugendförderung immer irgendwie wirkt, scheint banal (Lindner, 2009. S. 13). Evaluationen sollten daher den Blick auf Befunde richten, die nicht nur Verantwortliche in den Trägerschaften überzeugen, sondern auch den Fachpersonen der Kinder- und Jugendförderung Hinweise geben für eine reflexive und evidenzbasierte Praxis (Riedi, 2015). Eine Praxis mit „doppelter Reflexion“ (Lindenberg, 2005, S. 105) ist anzustreben: sowohl auf das professionelle Selbstverständnis wie auch auf die Sinndeutung des Gegenübers, insbesondere der Kinder und Jugendlichen. Was denken sie über Wirkung und Nutzen der Bildungsangebote, was über Lehrpersonen und Jugendarbeitende - sind das einfach alles ,Erwachsene“ oder gibt es Unterschiede? 


\section{Unterschiedliche Kompetenzen nutzen}

Während sich die Kinder- und Jugendverbände in der Regel an einer mehr oder weniger festen Mitgliedschaft orientieren und sich oftmals erlebnispädagogisch (z.B. draussen in der Natur) und/oder nach einer klar definierten Programmatik ausrichten, zeichnet sich die Offene Kinder- und Jugendarbeit durch ihre sozialräumliche, nicht an eine formale Mitgliedschaft gebundene Pädagogik aus und das Jugendhaus stellt ihren idealtypischen Aufenthaltsort dar. Gemeinsam ist beiden, dass zunehmend mögliche Zusammenarbeitsformen mit der Schule ausgelotet werden (bspw. Agharmiri, 2012; Bock \& Otto, 2007; Böllert, 2008; Chehata, 2014; Lischer, Kislig, Mosimann, Lauper, Lozano \& Egger, 2005).

Die schweizerische Strategie für eine Kinder- und Jugendpolitik betont die Notwendigkeit der Kinder- und Jugendförderung, denn der ,formale Bildungsbereich stösst (...) an seine Grenzen, wenn es um das Erlernen von sogenannten Soft-Skills wie Teamfähigkeit, Verantwortungsbereitschaft, Unternehmungsgeist und inter-kulturelle Kompetenzen geht, die im Sinne von Schlüsselkompetenzen für den heutigen Arbeitsmarkt betont werden (....) Es ist deshalb von Bedeutung, dass in Ergänzung zum Bildungs- und Ausbildungssystem adäquate ausserschulische Lernfelder gezielt gefördert und unterstützt werden“" (BSV, 2008, S. 8). Kinder- und Jugendförderung ist jedoch gefordert, nicht in die Rolle der stillen Zuarbeiterin für Kompetenzbereiche zu fallen, die „die Schule alleine nicht abdecken kann“ (Chehata, 2014, S. 36). Vielmehr geht es darum, gemeinsam mit der Schule ein Bildungsverständnis zu entwickeln, „das sich wieder den Subjekten zuwendet“" (ebd.). Es gilt insbesondere die unterschiedlichen Kompetenzen für gemeinsame Anliegen zu nutzen und so die Kinder- und Jugendförderung aus ihrer Rolle als „Erfüllungsgehilfen“" (Sturzenhecker, 2003, S. 47) zu entlassen.

In einem ersten Schritt entwickeln wir daher nachfolgend einen strukturtypisierenden Orientierungsrahmen für die Zusammenarbeit von Kinder- und Jugendförderung und Schule. Er dient als Ausgangspunkt für erste Folgerungen für ein Modell gelingender Zusammenarbeit.

\section{Orientierungsrahmen für die Zusammenarbeit}

Der strukturtypisierende Orientierungsrahmen für die Zusammenarbeit von Kinder- und Jugendförderung und Schule wird auf der Grundlage idealtypischer Praxen (Praktiken, Angebote oder Verfahren) sowie Konzeptionen von Sozialer Arbeit am Beispiel der Kinder- und Jugendförderung und Schule 
entwickelt (vgl. Tab. 1). Idealtypisch werden die Praxen und Konzeptionen genannt, weil sie in keinem der besprochenen Fälle tatsächlich trennscharf sind und auch im anderen Fall vorkommen können. Die Rahmung erfolgt anhand ausgewählter Kriterien. Ausgewählt wurden Kriterien, die sich als besonders relevant für das Handlungsfeld zeigen: Angebots-Prototypen, Angebots-Charakter, Kooperationsformen, Interventionsorientierung, Bildungskontexte und Bildungsziele. Die Kriterien werden mit Beispielen aus Schule und Kinder- und Jugendförderung illustriert.

Tabelle 1: Strukturtypisierender Orientierungsrahmen der Zusammenarbeit von Kinder- und Jugendförderung ( $\mathrm{K}+\mathrm{J}$ Förderung) und Schule

\begin{tabular}{|c|c|c|c|c|}
\hline & Schule & $\begin{array}{l}\text { Schule \& } \\
\text { K+J Förderung }\end{array}$ & $\begin{array}{l}\text { K+J Förderung } \\
\text { \& Schule }\end{array}$ & $\mathrm{K}+\mathrm{J}$ Förderung \\
\hline $\begin{array}{l}\text { Angebots } \\
\text { Prototypen }\end{array}$ & Mathematik & $\begin{array}{l}\text { Gewaltprävention } \\
\text { an Schulen }\end{array}$ & $\begin{array}{l}\text { Schweizer Ju- } \\
\text { gendfilmtage }\end{array}$ & Zeltlager \\
\hline $\begin{array}{l}\text { Angebots } \\
\text { Charakter }\end{array}$ & standardisiert & \begin{tabular}{|l|} 
teil- \\
standardisiert
\end{tabular} & strukturiert & \begin{tabular}{|l|} 
teil- \\
strukturiert \\
\end{tabular} \\
\hline $\begin{array}{l}\text { Kooperations- } \\
\text { form }\end{array}$ & keine & $\begin{array}{l}\text { Schulstandort be- } \\
\text { zogen }\end{array}$ & \begin{tabular}{|l} 
Lebenswelt be- \\
zogen
\end{tabular} & keine \\
\hline $\begin{array}{l}\text { Interventions- } \\
\text { orientierung }\end{array}$ & $\begin{array}{l}\text { schul- } \\
\text { pädagogisch }\end{array}$ & $\begin{array}{l}\text { sozialarbeiterisch / } \\
\text { sozialpädagogisch }\end{array}$ & \begin{tabular}{|l|} 
sozial- \\
pädagogisch / \\
soziokulturell \\
\end{tabular} & \begin{tabular}{|l} 
soziokulturell / \\
sozial- \\
pädagogisch \\
\end{tabular} \\
\hline $\begin{array}{l}\text { Bildungs- } \\
\text { kontext }\end{array}$ & formal & & & non-formal \\
\hline Bildungsziel & employability & & & citizenship \\
\hline
\end{tabular}

Quelle: eigene Darstellung

Es darf davon ausgegangen werden, dass Mathematik von Kindern und Jugendlichen deutlich mehr mit Schule als mit Freizeitbeschäftigung in Verbindung gebracht wird. Mathematik gilt daher im Modell als Angebots-Prototyp für den Bereich Schule. Auf der anderen Seite steht das Zeltlager als AngebotsPrototyp für Kinder- und Jugendförderung. Zeltlager ist für viele emotional verbunden mit Pfadi, Cevi, Jubla und anderen Jugendverbänden. Zwischen den beiden Angebotstypen liegt das Angebot Gewaltprävention an Schulen. Dieser Typus wird im Bereich der Kinder- und Jugendförderung entwickelt, aber spezifisch an Schulen angeboten. Das Primat der Durchführungsverantwortung liegt bei der Schule. Die Schweizer Jugendfilmtage stehen im Orientierungsschema für einen Angebotstypus, der klar im Bereich der Kinder- und Jugendförderung steht und das Durchführungsprimat innehat. Seit über 40 Jahren 
werden neben der Präsentation von Filmen junger Kunstschaffenden Workshops zur Erstellung eigener Kurzfilme zu einem vorgegebenen Thema verbunden mit einem Wettbewerb in verschiedenen Altersklassen angeboten. Dazu sind neben Kinder- und Jugendgruppen und Einzelpersonen explizit auch Schulklassen eingeladen (http://jugendfilmtage.ch/).

Der Angebots-Charakter variiert begriffstypisch zwischen standardisiert und strukturiert. Schule basiert auf gesetzlich geregelten Standards resp. Vereinheitlichungen. So sind bspw. Lehrpläne, Qualifikationen der Lehrpersonen sowie Ein- und Austritte in der Schule gesetzlich geregelt. Schulische Angebote werden in diesem Sinne als standardisiert typisiert. Die Kinderund Jugendförderung arbeitet demgegenüber mit sogenannten strukturierten Angeboten, die regelmässig und geplant angeboten werden, bei denen aber keine direkten Rechtsansprüche geltend gemacht werden können oder formale Bildungsqualifikationen erzielt werden.

Wo Kooperationsformen bestehen, können sie nach Deinet (o.J.) als schulstandort- oder lebensweltbezogen typisiert werden. Schulstandortbezogene Angebote fokussieren auf eine „Orientierung an Schülerinnen und Schülern (der Schule) [mit] Problem- und Einzelfallbezug“ (S. 13). Lebensweltbezogene Angebote hingegen fokussieren auf eine „Orientierung an Kindern und Jugendlichen [und auf] Themen und Problemen ihrer Lebenswelt“ (ebd.).

Die Interventionsorientierung der Schule kann als schulpädagogisch typisiert werden. Demgegenüber stehen Angebote der Sozialen Arbeit, die sozialarbeiterisch, sozialpädagogisch oder soziokulturell orientiert sind (zur näheren Unterscheidung siehe Husi \& Villiger, 2012). Die sozialarbeiterische/sozialpädagogische Orientierung zeigt sich bei Angeboten, die schulstandortbezogen einen stärkeren Problem- und Einzelfallbezug aufweisen. Die sozialpädagogische/soziokulturelle Orientierung zeigt sich bei Angeboten, die Bildungsprozesse in eigens dazu entwickelten Workshops anbieten und auf lebensweltlich ausgerichtete Interessen der Kinder- und Jugendlichen fokussieren. Die soziokulturelle/sozialpädagogische Orientierung steht bei Freizeitaktivitäten im Vordergrund, die eine hohe Partizipation (Mitwirkung und Mitbestimmung bis hin zu Mitverantwortung) der Beteiligten voraussetzen.

Fachlich wird zuweilen zwischen formaler (curricular organisierter und verpflichtender), non-formaler (organisierter aber freiwilliger) und informeller (ungeplanter und nichtintendierter) Bildung unterschieden (Rohlfs, 2011, S. 35-42). Da diese Prozesse in allen Bildungssituationen in der einen oder anderen Art stattfinden, bevorzugen wir die Terminologie Bildungskontexte (kritisch dazu Bock \& Otto, 2007, S. 207), die idealtypisch die eine oder andere Form repräsentieren: formaler Kontext (Schule) und non-formaler Kontext (Kinder- und Jugendförderung). Informelle Bildung wäre in diesem Konstrukt dem Kontext Familie zuzuordnen. In eher formalen Bildungskontexten 
werden Bildungsprozesse von Lehrpersonen oder Fachpersonen Sozialer Arbeit angeleitet. Demgegenüber sind in eher non-formalen Bildungskontexten Kinder- und Jugendliche sowohl Adressatinnen und Adressaten der Angebote wie auch Akteurinnen und Akteure in eigener Sache. Dies zeigt sich in der Schweiz besonders deutlich daran, dass in Jugendverbänden die Leiterinnen und Leiter im Vergleich zu anderen Ländern sehr jung sind: „Bereits mit 14 leitet eine Gruppenführerin in der Pfadi ein Fähnli mit 6 bis 8 Mädchen (...), mit 18 kann man ein Zeltlager mit 20 bis 30 Kindern leiten“ (Steiner, 2017).

Schule und Kinder- und Jugendförderung werden abschliessend im Modell unter dem Kriterium Bildungsziel eingeordnet. Idealtypisch stehen bei der Schule die Qualifizierung für die Erwerbsarbeit und bei der Kinder- und Jugendförderung die Subjektbildung im Sinne von mündigen Bürgerinnen und Bürgern als Bildungsziele im Vordergrund. Bildungspolitisch werden diese Orientierungen auch als employability und citizenship bezeichnet. Sie umfassen instrumentelle, interpersonale sowie systemische Kompetenzen und wären künftig vermehrt miteinander abzustimmen (vgl. http://www.unideusto.org/ tuningeu/competences.html).

\section{Implikationen für die Zusammenarbeit}

Mit den im Orientierungsrahmen zur Diskussion gestellten Kriterien können Implikationen für die Zusammenarbeit von Kinder- und Jugendförderung und Schule skizziert werden. An verschiedenen Orten besteht bereits eine langjährige Zusammenarbeit. An anderen werden Möglichkeiten (und Instrumentalisierungen) dieser Zusammenarbeit bildungspolitisch erst jetzt so richtig erkannt. Der Orientierungsrahmen dient dazu, sowohl in der Planung als auch in der Durchführung der Zusammenarbeit die Stärken, Besonderheiten, Kompetenzen und die Möglichkeiten der Schule oder der Kinder- und Jugendförderung zu kennen, zu reflektieren und für beide Seiten nutzbringend einzusetzen. Allenfalls sind in der einen oder anderen Zusammenarbeit nicht alle der von uns ausgewählten Kriterien gleichermassen relevant für die gemeinsame Orientierung. Und möglicherweise zeigt sich in der konkreten Zusammenarbeit, dass das eine oder andere Kriterium neu hinzuzunehmen ist.

Zentral bleibt dabei aber immer die Frage, ob es in der fachlichen $\mathrm{Zu}$ sammenarbeit gelingt, ein gemeinsames Bildungsverständnis zu entwickeln, das die Besonderheiten des jeweilig anderen anerkennen kann. Damit nicht mehr gilt: „Alles, was Schule anfasst, wird zu Schule!“ (Deinet, o.J., S. 3). Und es ist auch zu fragen, wie Kinder und Jugendliche die jeweiligen Bildungskonzeptionen deuten, wie sie sich diese aneignen und welches Mehr an Standardisierung in der Kinder- und Jugendförderung resp. welches Mehr an 
Partizipation in der Schule sie sich tatsächlich wünschen. Denn McDonald's ist nicht aus sich heraus die ,erfolgreichste“ Jugendeinrichtung - sie wird es nur durch Sinndeutung anderer.

\section{Literatur}

Agharmiri, K. (2012). „Wenn die Sozialpädagogen da sind, muss man nix lernen“ Die Aneignung eines schulbezogenen Angebots der Jugendhilfe zum sozialen Lernen aus der Perspektive von Grundschulkindern. In R. Braches-Chyrek, G. Lenz \& B. Kammermeier (Hrsg.), Soziale Arbeit und Schule. Im Spannungsfeld von Erziehung und Bildung (S. 157-174). Opladen: Barbara Budrich.

Bock, K. \& Otto, H.-U. (2007). Die Kinder- und Jugendhilfe als Ort flexibler Bildung. In M. Harring, C. Rohlfs \& C. Palentien (Hrsg.), Perspektiven der Bildung. Kinderund Jugendliche in formellen, nicht-formellen und informellen Bildungsprozessen (S. 203-217). Wiesbaden: VS Verlag für Sozialwissenschaften.

Böllert, K. (2008). Bildung ist mehr als Schule - Zum kooperativen Bildungsauftrag von Familie, Schule, Kinder- und Jugendhilfe. In K. Böllert (Hrsg.), Von der Delegation zur Kooperation (S. 7-31). Wiesbaden: VS Verlag für Sozialwissenschaften.

BSV Bundesamt für Sozialversicherung. (2008). Strategie für eine Schweizerische Kinder- und Jugendpolitik. Bern: EDI Eidgenössisches Departement des Innern.

Chehata, Y. (2014). Jugendarbeit an und in Grenzen - Kooperationen von Jugendarbeit und Schule. deutsche jugend, 62 (1), 28-37.

Chiapparini, E. (2017). Schule und Freizeit. Partizipative Angebote für Kinder und Jugendliche. punktum (5), 12-14.

Deinet, U. (2015). Wohin entwickelt sich die Kinder- und Jugendförderung? Referat gehalten an der Delegiertenversammlung der okaj zürich am 26.05.2017. Abgerufen am 14.08.2017 unter:

http://www.okaj.ch/angebote/aus-und-weiterbildung/downloads-zuweiterbildungen-1

Deinet, U. (o.J.). Kooperation aus Sicht von Kindern und Jugendlichen, Schulen und Jugendhilfe - Schnittmengen und Unvereinbarkeiten. Abgerufen am 17.08.2017 unter:

http://ganztag-blk.de/ganztags-box/cms/upload/sozialraumorientierung/pdf/1a/2.

DEINET Kooper.Kindersicht.pdf

Gerodetti, J. \& Schnurr, S. (2013). Offene Kinder- und Jugendarbeit in der Schweiz. In U. Deinet \& B. Sturzenhecker (Hrsg.), Handbuch Offene Kinder- und Jugendarbeit (S. 827-839). Wiesbaden: VS Verlag für Sozialwissenschaften.

Husi, G. \& Villiger, S. (2012). Sozialarbeit, Sozialpädagogik, Soziokulturelle Animation. Theoretische Reflexion und Forschungsergebnisse zur Differenzierung Sozialer Arbeit. Luzern: interact.

KKJF Konferenz der kantonalen Beauftragten für Kinder- und Jugendförderung (2010). Standards der Kinder- und Jugendförderung Schweiz. Positionspapier. Abgerufen am 07.07.2017 unter: 
http://www.sodk.ch/fileadmin/user_upload/Fachbereiche/Kinder_und_Jugend/B_ 2010_KKJF_Standards_KJF.pdf

Krummenacher, J. (2014). Historische Betrachtung zur Entwicklung der Jugendverbände. In S. Gretler Heusser \& P. Stade (Hrsg.), Verbandsjugendarbeit in der Schweiz: Herausforderungen und Entwicklungen gestern, heute und morgen (S. 13-44). Luzern: interact.

Lindenberg, M. (2005). „Ohne Goffman hätte ich es im Knast nicht ausgehalten“. In W. Braun \& M. Nauerth (Hrsg.), Lust an der Erkenntnis. Zum Gebrauchswert soziologischen Denkens für die Praxis Sozialer Arbeit (S. 81-105). Bielefeld: Kleine-Verlag.

Lindner, W. (2009). Kinder- und Jugendarbeit wirkt. Aber wie und wo und warum genau? In W. Lindner (Hrsg.), Kinder- und Jugendarbeit wirkt. (2. Aufl., S. 918). Wiesbaden: VS Verlag für Sozialwissenschaften.

Lischer, R., Kislig, R., Mosimann, E., Lauper, S., Lozano, D. \& Egger, C. (2005). Offene Jugendarbeit und Schule. Ein Leitfaden für die Zusammenarbeit. Worb: VOJA Vernetzte Offene Jugendarbeit Kanton Bern. Abgerufen am 15.08.2018 unter: http://www.voja.ch/download/leitfaden_jugendarbeit_und_Schule.pdf

Marti, P. (2011). Theorie der Jugendarbeit. Arbeitshilfen für die Jugendarbeit. ZürichWipkingen: jugendarbeit.ch. Abgerufen am 16.10.2017 unter: http://www.jugendarbeit.ch/download/jar_ueberblick.pdf

Riedi, A. M. (2015). Dialogische Praxis- und Wissensentwicklung in der Sozialen Arbeit. In Zusammenarbeit mit Esther Forrer und Karin Werner. Zürich: ZHAW Soziale Arbeit. Abgerufen am 16.08.2017 unter: https://digitalcollection.zhaw.ch/handle/11475/1176

Riedi, A. M. \& Biebricher, M. (2013). Jugendarbeit Wallisellen. Bestandessichtung, Analyse und Empfehlungen. Schlussbericht. Dübendorf: ZHAW Soziale Arbeit.

Riedi, A. M. \& Stohler, R. (2013). Jugendnetz Siggenthal. Analyse und Empfehlungen zur Weiterentwicklung von Schulsozialarbeit, Jugendarbeit und Jugendnetz. Schlussbericht. Dübendorf: ZHAW Soziale Arbeit.

Rohlfs, C. (2011). Bildungseinstellungen. Wiesbaden: VS Verlag für Sozialwissenschaften.

Schenker, D. \& Wettstein, H. (2013). Soziokulturelle Animation und Jugendarbeit. In S. Huber \& P. Rieker (Hrsg.), Offene Kinder- und Jugendarbeit in der Schweiz (S. 44-61). Weinheim: Beltz Juventa.

Spierts, M. (1998). Balancieren und Stimulieren. Methodisches Handeln in der soziokulturellen Animation. Luzern: Verlag für Soziales und Kulturelles.

Steiner, R. (2015). Referat vom 28.11.2015 zum Abschlussevent der Jubiläumskampagne ,Kinder- und Jugendförderung wirkt! - 90 Jahre im Kanton Zürich“ der okaj Zürich. Unveröffentlichte Rede.

Steiner, R. (2017). Vortrag vom 31.05.2017 anlässlich der Vernissage zur Publikation „Alles was Recht ist" der okaj Zürich. Unveröffentlichte Rede.

Sturzenhecker, B. (2003). Zum Bildungsanspruch von Jugendarbeit. Mitteilungen des Landesjugendamtes Westfalen-Lippe (153), 47-61.

Torresan, S., Cabral, A., Tobler, M. \& Rommel, M. (2015). Mehr als ein Ja oder Nein. Eine Entdeckungsreise in die Welt des ältesten Jugendhauses der Schweiz: das Juhu in Winterthur. Winterthur: Juhu Winterthur. 
III. Perspektiven aus der Praxis 



\title{
„Kann ich mal mit jemandem reden?“ Berufsintegration und Sozialpädagogik in der Schule in Kleingruppen Dielsdorf
}

\author{
Joël Humbert-Droz
}

Angebote der Sozialpädagogik, wie beispielsweise Berufsintegration, zählen zu den Kernelementen der Schule in Kleingruppen Dielsdorf. Deshalb geht der folgende Beitrag praxisnah der Frage nach, wie sich die sozialpädagogischen Angebote in den letzten Jahren verändert haben und wie diese im gegenwärtigen Konzept integriert sind.

\section{Die Schule in Kleingruppen Dielsdorf - Kurzbeschreibung}

Die Schule in Kleingruppen Dielsdorf ist eine kommunale, von der Bildungsdirektion des Kantons Zürich anerkannte Tagessonderschule. Sie wird seit 1989 von der Sekundarschule Dielsdorf-Regensberg-Steinmaur geführt. Die Zielgruppe der Schule in Kleingruppen Dielsdorf besteht aus normalbegabten Mädchen und Knaben im Mittelstufen- und Sekundarschulalter mit Beziehungs-, Verhaltens- und Lernschwierigkeiten, welche im Rahmen der Volksschule nicht angemessen gefördert werden können. Die Schule führt zwei Schulklassen mit je sechs Schülern und Schülerinnen. Sämtliche Schüler und Schülerinnen besitzen einen Status der Sonderschulbedürftigkeit ${ }^{1}$. Dieser Status bedeutet, dass diese Schülerinnen und Schüler zusätzliche Unterstützung durch schulische Heilpädagogik sowie eine individuelle Unterstützung beim Berufswahlprozess durch Sozialpädagoginnen und Sozialpädagogen erhalten.

Die Hauptelemente des schulischen Konzeptes zielen auf drei Förderbereiche: auf den Bereich des sozialen Verhaltens der Kinder und Jugendlichen, auf eine mögliche Reintegration in die Regelschule und auf die Unterstützung beim Berufswahlprozess (vgl. KGS, 2017).

1 Der Status der Sonderschulbedürftigkeit kann durch eine schulpsychologische Abklärung bzw. durch einen schulpsychologischen Dienst ermittelt werden. 


\section{Einführung der Sozialpädagogik und Berufsintegration}

Die Ansprüche, diese drei Förderbereiche fachlich und differenziert bearbeiten zu können, haben sich im Kanton Zürich stetig weiterentwickelt und dazu geführt, dass sich viele Sonderschulen spezialisiert haben, um diesen Ansprüchen und Vorgaben des Kantons gerecht zu werden. Für die Schule in Kleingruppen Dielsdorf bedeutete dies einen kontinuierlichen Ausbau des Schulteams und einen Stellenausbau bezüglich Interdisziplinarität, was auch die Beiträge der Sozialen Arbeit begünstigen konnte. Heute setzt sich das Schulteam aus einer Schulleitung, zwei Lehrpersonen, einer schulischen Heilpädagogin und zwei Sozialpädagogen (einer im Bereich Sozialpädagogik und der andere im Bereich Berufsintegrationscoaching) zusammen.

Die Begleitung der Jugendlichen bei der Berufsintegration gewann in den letzten Jahren an Wichtigkeit. Das Finden einer für den einzelnen Jugendlichen bzw. die einzelne Jugendliche jeweils entsprechenden Anschlusslösung nach der obligatorischen Schulzeit ist für alle Beteiligten von großer Bedeutung - nicht zuletzt auch für die einweisenden Stellen, welche zudem die finanzielle Seite einer Sonderschulplatzierung zu beachten haben.

Mit der Einführung der Sozialpädagogik setzte sich die Schule in Kleingruppen Dielsdorf zum Ziel, den von vielen sozialen Themen begleiteten Prozess der Berufswahl fest in den Schulalltag einzuflechten. Weiter erhöhen seit Juli 2013 auch kantonale Vorgaben die Wichtigkeit der Berufsintegration. So erhalten auch integrierte Sonderschülerinnen und -schüler an einer Regelschule ab der ersten Sekundarstufe Einzelcoachings in der Berufsintegration (vgl. Bildungsdirektion Kanton Zürich, 2013, S. 11). Durch diese Vorgabe werden nach dem aktuellen Konzept der Schule in Kleingruppen Dielsdorf auch die integrierten Sonderschülerinnen und -schüler der Sekundarschule durch das Berufsintegrationscoaching der Schule in Kleingruppen Dielsdorf unterstützt. Zusätzlich entspricht es auch dem Anspruch der Schule in Kleingruppen Dielsdorf, dass die Jugendlichen besonders in der Anfangszeit einer Ausbildung begleitet werden können. Es findet also auch eine Nachbetreuung statt. Dies alles erfordert personelle Ressourcen und hat dazu geführt, dass das Schulteam um eine weitere Sozialpädagogik-Stelle ausgebaut wurde. Erst so konnte die zuerst eingeführte sozialpädagogische Stelle verstärkt auf das Berufsintegrationscoaching konzentriert werden. Aufgrund der vielen Schulplatzanfragen und des immer grösser werdenden Bedarfs bei der Unterstützung in der Berufswahl wurde diese zusätzliche Stelle von der Schulpflege diskussionslos bewilligt.

Folgend wird nun genauer auf das aktuelle Konzept eingegangen. Der Fokus liegt hierbei auf den Beiträgen der Sozialpädagogik. 


\section{Das gegenwärtige Konzept der Schule in Kleingruppen Dielsdorf}

Wie bereits im Vorfeld beschrieben, setzt sich die Schule in Kleingruppen Dielsdorf aus einem interdisziplinären Team und zwei Schulklassen mit je sechs Schülerinnen und Schülern zusammen. Der eher kleine Rahmen der Schule in Kleingruppen Dielsdorf und die niedrige Anzahl an Schülern und Schülerinnen schaffen eine kinderzentrierte Atmosphäre. Zudem können sich je nach Situation die Aufgaben des Fachpersonals überschneiden. Dies erfordert ein hohes Mass an Austausch innerhalb des Teams. Tägliche Briefings und wöchentliche Teamsitzungen gewähren diesen Austausch. Mit dem Bewusstsein, dass es zu Überschneidungen kommen kann, sind die Aufgabenund Verantwortungsbereiche gemäss dem Konzept der Schule in Kleingruppen Dielsdorf klar aufgeteilt. Auf den schulischen Unterricht bezogen, zeigt sich dies z.B. bei der Aufteilung der Schulfächer unter den Lehrkräften. Im Bereich der Sozialpädagogik haben sich zwei unterschiedliche Gebiete entwickelt. So werden sozialpädagogische Stelle und Berufsintegrationscoaching getrennt voneinander koordiniert, wenn auch in beiden Bereichen je ein Sozialpädagoge tätig ist. Beide Stellen arbeiten zusammen und haben regelmässige gemeinsame Planungsgefässe. Bis auf einige Überschneidungen im schulischen Alltag unterscheiden sich die Aufgabengebiete zwischen der sozialpädagogischen Stelle und dem Berufsintegrationscoaching jedoch klar, worauf in den zwei folgenden Abschnitten eingegangen wird.

\section{Sozialpädagogik in der Schule in Kleingruppen Dielsdorf}

Die Verantwortungsbereiche der sozialpädagogischen Stelle sind in der Schule in Kleingruppen Dielsdorf unterschiedlich und lassen sich in drei Arbeitsbereiche unterteilen:

Eine Hauptaufgabe besteht aus der direkten Begleitung und Unterstützung der Schüler und Schülerinnen, aber auch der Lehrperson im Unterricht und soll im Folgenden mit drei Methoden und je einem ausgewählten Fallbeispiel veranschaulicht werden:

a) Schulische Unterstützung nach Vorgabe:

Die Lehrperson erkennt, dass Peter $^{2}$ bei den Recherchen für seinen Vortrag über Elefanten mehr Anleitung benötigt. Gemeinsam wird vereinbart, dass

2 Bei den verwendeten Namen der Schülerinnen und Schüler im Text handelt es sich um Decknamen. 
die sozialpädagogische Fachperson zusammen mit Peter ein Mindmap erstellt, um die Themen zu definieren, welche Peter dann bei seinem Vortrag über Elefanten vorstellen möchte. Weiter kann Peter dann beim Recherchieren zu diesen Themen in Büchern oder im Internet direkt unterstützt und angeleitet werden.

\section{b) Sozialpädagogische Intervention in Form von Aktivierungsspielen:}

Die Lehrperson und die sozialpädagogische Fachperson stellen fest, dass gewisse Schülerinnen oder Schüler müde wirken und die Klassendynamik eher als träge beschrieben werden kann. Spontan wird entschieden, mit der Klasse auf den Pausenplatz zu gehen und unter Anleitung des Sozialpädagogen ein Tischtennis-Rundlauf zu spielen.

\section{c) Bearbeitung von Konflikten im schulischen Alltag:}

Peter und Felix kommen am Morgen zerstritten und schlecht gelaunt in die Schule. Die Stimmung ist geladen und lässt einen Unterricht kaum zu. Die sozialpädagogische Fachperson zieht sich mit den Beiden in ein Büro zurück und versucht mit ihnen gemeinsam nach Lösungen für ihren Konflikt zu suchen.

Ein zweiter Aufgabenbereich besteht darin, soziale Förderziele mitzuentwickeln, zu koordinieren und deren Erreichung zu unterstützen. Hierbei geht es zusammenfassend um die soziale Förderplanung (vgl. Hollenweger, 2009) ${ }^{3}$. Die Mitentwicklung und Begleitung bei der Umsetzung der sozialen Ziele findet in Zusammenarbeit mit den Fallverantwortlichen und den Eltern statt, indem Elemente des Case Managements (vgl. Stangl, 2017) beigezogen werden. ${ }^{4}$ Dieser Aufgabenbereich soll hier anhand von zwei Methoden und je einem ausgewählten Fallbeispiel aus der Praxis ebenfalls veranschaulicht werden:

\section{a) Mobilitätstraining:}

Sandra wird im nächsten Semester Schnupperlehren absolvieren und muss dafür oft Zug oder Bus fahren. Sie fühlt sich im Umgang mit den öffentlichen

3 Die soziale Förderplanung der Schule in Kleingruppen Dielsdorf orientiert sich an den zehn Lebensbereichen ICF (International Classification of Functioning): Allgemeines Lernen; Mathematisches Denken; Spracherwerb und Begriffsbildung; Lesen und Schreiben; Umgang mit Anforderungen; Kommunikation; Bewegung und Mobilität; Für sich selber sorgen; Umgang mit Menschen; Freizeit, Erholung und Gemeinschaft (vgl. Hollenweger, 2009).

4 Die Schule in Kleingruppen Dielsdorf arbeitet mit Elementen des Case Managements, einer Methode zur strukturierten und koordinierten Gestaltung von Unterstützungs- und Beratungsprozessen, die den Betroffenen die individuell benötigten Dienstleistungen zur Lösung ihrer Probleme zugänglich machen möchte (vgl. Stangl, 2017). Die Verantwortung über Aktenführung und Organisation von Elterngesprächen obliegen den jeweilig zugeteilten Lehrkräften oder sozialpädagogischen Fachpersonen. 
Verkehrsmitteln unsicher. Als Förderziel wird vereinbart, dass Sandra von der sozialpädagogischen Fachperson dabei unterstützt wird, den Umgang mit dem Fahrplan und die Planung von Reisen zu erlernen und anfänglich bei Übungsreisen begleitet wird.

\section{b) Unterstützung bei der Suche nach passenden Freizeitaktivitäten:}

Tim und seine Eltern wohnen noch nicht lange in der Schweiz und teilen bei einem Gespräch mit, dass sie sich leider noch nicht so gut auskennen, sich aber für den sportbegeisterten Tim eine regelmässige Freizeitbeschäftigung wünschen. Die sozialpädagogische Fachperson unterstützt die Eltern und Tim dabei, seine Interessen zu erkunden, hilft mit, einen Judo-Schnupperkurs zu organisieren und bespricht mit ihnen eine Liste von verschiedenen regionalen Vereinen.

Ein dritter Arbeitsbereich der sozialpädagogischen Stelle ist die Erlebnispädagogik. Wöchentlich ist jeweils ein Nachmittag erlebnispädagogischen Inhalten gewidmet. Hier bedient sich die Sozialpädagogik der Schule in Kleingruppen Dielsdorf gerne des Ansatzes von Heckmaier und Michl (1994): In diesem wird die Erlebnispädagogik als handlungsorientierte Methode betrachtet, bei welcher die Elemente Natur, Erlebnis und Gemeinschaft pädagogisch zielgerichtet miteinander verbunden werden und die zwischenmenschliche Begegnung und Beziehung begünstigen kann (vgl. Heckmaier, 1994).

Eine wesentliche Arbeitseigenschaft des Sozialpädagogen ist es auch, dass er sich vom ordentlichen Stundenplan loslösen kann und so die Möglichkeit hat, Aufgaben, die sich durch die interdisziplinäre Arbeit ergeben, in Absprache mit den Fallverantwortlichen zu übernehmen. Dazu gehören beispielsweise Gespräche mit Therapeuten und Therapeutinnen von Schülerinnen oder Schülern, Begleitung in die Logopädie oder Besuche bei Mittagstischen, weil viele Schülerinnen und Schüler der Schule in Kleingruppen Dielsdorf sich zum Mittagessen bei privaten Familien befinden, die sich bei der Ortsgemeinde zur Verfügung stellen.

\section{Berufsintegrationscoaching in der Schule in Kleingruppen Dielsdorf}

Das Berufsintegrationscoaching der Schule in Kleingruppen Dielsdorf setzt sich zum Ziel, die Jugendlichen bestmöglich auf den Übergang in das „Erwachsenenleben“ vorzubereiten und ihnen zu einer adäquaten Anschlusslösung zu verhelfen. Dies kann eine Berufslehre mit einem Eidgenössischen Fähigkeitszeugnis (EFZ), einem Eidgenössischen Berufsattest (EBA) oder 
eine Praxisausbildung (PrA) auf dem ersten oder zweiten Arbeitsmarkt sein ${ }^{5}$. Zudem kann eine adäquate Anschlusslösung auch aus einem Praktikumsjahr oder aus einem der vielen Brückenangebote ${ }^{6}$ bestehen. Das Berufsintegrationscoaching richtet sich an Schülerinnen und Schüler der Schule in Kleingruppen Dielsdorf und an die integrierten Sonderschülerinnen und -schüler der Sekundarschule Dielsdorf. Die Lernenden haben wöchentlich bis zu zwei Schullektionen Anspruch auf eine Einzelberatung. Je nach Bedarf kann die Anzahl der Lektionen auch erhöht werden. Der Auftrag der Schule in Kleingruppen Dielsdorf beinhaltet auch die Nachbetreuung der Jugendlichen. Dies bedeutet, dass den Betrieben, den Eltern und auch den Jugendlichen bei Bedarf während der Lehrzeit eine Unterstützung zur Verfügung steht. Die Zusammenarbeit mit den Eltern wird für den gesamten Prozess der Berufswahl als ein zentrales Element betrachtet.

$\mathrm{Zu}$ den Hauptaufgaben des Berufsintegrationscoachings gehören:

- Berufsfindungsprozess fördern, unterstützen, koordinieren.

- Zusammenarbeit mit den Eltern, Lehrkräften und der Sozialpädagogik.

- Coaching bei der Suche von Schnupperlehrstellen und Lehrstellen sowie beim Erstellen der Bewerbungsunterlagen.

- Aufbau und Pflege eines Netzwerkes von Schnupper- und Lehrbetrieben.

- Regelmässige Auswertungsgespräche in den Schnupper- bzw. Lehrbetrieben.

- Orientierungsgespräche bei potentiellen Lehrbetrieben zum Modell Schule in Kleingruppen Dielsdorf.

- Besuch relevanter Anlässe/Stellen (Berufsmesse; Berufsbesichtigungsanlässe; Berufsinformationszentrum etc.).

- Lehrlingscoaching (Nachbetreuung).

Die verschiedenen Aufgaben können in die Bereiche Coaching (a) und Netzwerk (b) unterteilt werden.

\section{a) Coaching:}

Die Form und die Inhalte des Coachings werden den Schülerinnen und Schülern individuell angepasst. So kann es sein, dass bei Peter ein guter Zugang

5 In der Schweiz wird zwischen dem ersten und dem zweiten Arbeitsmarkt unterschieden. Während bei beiden Arbeitsmärkten Berufslehren mit den Abschlüssen EFZ (Eidgenössisches Fähigkeitszeugnis) bzw. EBA (Eidgenössisches Berufsattest) absolviert werden können, werden die Lernenden auf dem zweiten Arbeitsmarkt zusätzlich durch ein Coaching unterstützt und es wird im Gegensatz zum ersten Arbeitsmarkt der Abschluss PrA (Praxisausbildung) angeboten. Zudem sind die Arbeitsplätze des zweiten Arbeitsmarktes geschützt.

6 Brückenangebote geben Schulabgängerinnen und Schulabgängern die Möglichkeit, während eines oder zwei Semestern gezielt schulische Lücken aufzuarbeiten, sich bei der $\mathrm{Zu}$ kunftsplanung durch Fachleute unterstützen zu lassen und eine berufliche oder gymnasiale Anschlusslösung zu finden und sich darauf vorzubereiten. 
durch praktische Aufgaben entsteht, während mit Sandra verstärkt mit Gesprächen und auf theoretischer Ebene gearbeitet wird. Das Berufsintegrationscoaching besitzt diesbezüglich die Freiheit, mit unterschiedlichen methodischen Ansätzen zu arbeiten, wobei klar gesagt werden kann, dass sich die Methoden im Bereich der Kompetenzorientierung befinden und ressourcenund lösungsorientierte Techniken eingesetzt werden (vgl. Cassée, 2007).

b) Netzwerk:

Die integrierten Sonderschülerinnen und -schüler an einer Regelschule der Sekundarschule Dielsdorf werden vor Ort beraten; denn der Weg in die Schule in Kleingruppen Dielsdorf würde für die jeweiligen Schüler und Schülerinnen der Sekundarschule zu viel Zeit in Anspruch nehmen. Das eben erwähnte Coaching jener Schülerinnen und Schüler und die Pflege des Netzwerkes mit Lehr- und Schnupperbetrieben sowie das Lehrlingscoaching in den Ausbildungsbetrieben gehören zum Aufgabenbereich des Netzwerkes. Diese Netzwerkarbeit führt dazu, dass sich das Berufsintegrationscoaching und damit der Sozialpädagoge oft ausserhalb der Schule in Kleingruppen befinden, was zu Herausforderungen führen kann (vgl. 7).

\section{6. Überschneidung der Bereiche Berufsintegrationscoaching und sozialpädagogische Stelle}

Die Auseinandersetzung der Schülerinnen und Schüler mit der eigenen $\mathrm{Zu}$ kunft wird von vielen sozialen Themen begleitet. Für das Berufsintegrationscoaching ist es deshalb vorteilhaft, die Jugendlichen persönlich zu kennen und die Schülerinnen und Schüler auch im schulischen Alltag zu erleben. Hier überschneiden sich sozialpädagogische Stelle und Berufsintegrationscoaching, indem das Berufsintegrationscoaching regelmässig die Funktion der sozialpädagogischen Stelle übernimmt und wöchentlich acht Lektionen unterstützend im Unterricht anwesend ist. Dies ermöglicht dann wiederum der sozialpädagogischen Stelle, Aufgaben außerhalb des Unterrichts wahrzunehmen. Zudem war es ein Bedürfnis der Schule in Kleingruppen Dielsdorf, einen Berufsintegrationscoach mit handwerklichem Hintergrund anzustellen, der die Verantwortung für den schuleigenen Werkunterricht übernimmt und dadurch einen weiteren Kanal zur Schülerschaft gewinnt. Das Berufsintegrationscoaching erhält somit die Möglichkeit, vieles über die Persönlichkeiten und Ressourcen der Schülerinnen und Schüler zu erfahren und auch praktische und soziale Kompetenzen kennen und einschätzen zu lernen, was dann wiederum für das Einzelcoaching wichtig sein kann. 


\section{Herausforderungen des Konzepts der Schule in Kleingruppen}

Von den eigenen Erfahrungswerten ausgegangen, siedeln sich die Herausforderungen auf unterschiedlichen Gebieten an, von denen vier als besonders zentral erachtet werden können:

a) Kommunikation:

Die Aufteilung in die verschiedenen Aufgabengebiete und die jeweiligen Überschneidungen erfordern ein hohes Mass an Kommunikationsmöglichkeiten. Um dieser Tatsache entgegenzutreten, besitzt die Schule in Kleingruppen Dielsdorf verschiedene Austauschgefässe. Die täglichen Briefings und wöchentlichen Teamsitzungen können mit einem grossen zeitlichen Aufwand einhergehen. Vor allem der Austausch zwischen den Lehrkräften und dem Berufsintegrationscoach muss gut geplant sein, da sich das Berufsintegrationscoaching wie bereits erwähnt oft nicht im Schulgebäude befindet. Zusätzlich bedient sich das Schulteam auch Hilfsmitteln wie z.B. Chat-Gruppen oder elektronischer Wolken (Clouds), um den Informationsaustausch zu vereinfachen.

b) Unterschiedliche Berufsgruppen und Berufskulturen:

Die unterschiedlichen Arbeitsbereiche und die damit einhergehenden, zu verfolgenden Ziele können zu unterschiedlichen Ansichten innerhalb des Teams führen. Es kann auch in gewisser Weise von einem „Konstruktiven Ringen“ zwischen den Berufsgruppen gesprochen werden. Hier zeigt sich klar, dass die Lehrkräfte ihre Ziele im Bereich der Wissensvermittlung zu verfolgen haben und deshalb auch den Fokus verstärkt auf schulische Inhalte richten müssen. Die Sozialpädagogen der sozialen Stelle und des Berufsintegrationscoachings hingegen neigen dazu, den Fokus eher auf die sozialen Ziele zu richten. Beide Disziplinen müssen sich dieser Tatsache bewusst sein und sich für eine Konsensfindung einsetzen.

c) Evaluation und Nachhaltigkeit:

Die Schule in Kleingruppen Dielsdorf ist mithilfe von verschiedenen Fragebögen für Eltern, Behörden und andere Mitarbeitende darum bemüht, die Qualität ihrer Arbeit zu reflektieren. Allerdings bleibt es eine Herausforderung, die Wirksamkeit der eigenen Arbeit zu evaluieren. Regelmässige Treffen mit ehemaligen Schülerinnen und Schülern, die Dimension der Beteiligung und die Qualität der Kontakte zu ehemaligen Schülerinnen und Schülern oder auch zu deren Eltern können es aber zulassen, Rückschlüsse zu ziehen.

d) Nachhaltigkeit Berufsintegrationscoaching:

Das Berufsintegrationscoaching besitzt durch die Nachbetreuung (Lehrlingscoaching) regelmässigen Kontakt zu den Lernenden, Wissen über die 
jeweiligen aktuellen Lebensumstände und die Übersicht der jeweiligen Lehrbetriebe. Im Rahmen der Nachbetreuung ist es auch schon vorgekommen, dass es während einer laufenden Ausbildung zu einem Berufswechsel oder gar zu einem Lehrstellenabbruch gekommen ist. Gründe für solche Richtungswechsel sind meistens sehr komplex. Für das Berufsintegrationscoaching ist es hierbei wichtig, solche Richtungswechsel mit den Lernenden differenziert zu reflektieren. Die Frage, wie gross schlussendlich der Einfluss des Berufsintegrationscoachings ist, scheint schwierig zu beantworten zu sein. Das Berufsintegrationscoaching hat sich aber zum Ziel gesetzt, für die statistische Erfassung des beruflichen Weiterverlaufs von Schülerinnen und Schülern der Schulen in Kleingruppen ein geeignetes Werkzeug zu entwickeln.

\section{Möglichkeiten und Chancen des Konzepts}

Die Beiträge der Sozialpädagogik prägen das heutige Konzept der Schule in Kleingruppen in Dielsdorf, das in Zusammenarbeit mit dem gesamten Team entwickelt wurde und stetig an die neuen Anforderungen und Herausforderungen angepasst wird. Es legt strukturelle Austauschgefässe fest, um eine erfolgreiche Zusammenarbeit zu begünstigen. Die Interdisziplinarität des Teams erlaubt eine differenziertere Arbeit mit den individuellen Themen der einzelnen Schülerinnen und Schüler. Weitere Chancen des Konzepts zeichnen sich in folgenden Punkten besonders klar ab:

a) Durch die enge Zusammenarbeit im Team und die unterstützende Funktion der sozialpädagogischen Methoden während des Unterrichts erhöhen sich die Chancen, den Bedürfnissen der Schülerschaft gerecht zu werden. Dadurch, dass sich die Sozialpädagogik losgelöst vom Unterricht bewegen kann, können die Schülerinnen und Schüler intensiver bei der Bearbeitung ihrer individuellen Förderziele unterstützt werden.

b) Das regelmässige Einzelcoaching ermöglicht eine sehr individuelle Arbeitsweise. Lehrmittel, Werkzeuge und theoretische Handlungsansätze können so je nach Bedürfnissen eingesetzt und angepasst werden.

c) Eine weitere Chance besteht darin, dass es den Lehrkräften durch die enge Zusammenarbeit mit dem Berufsintegrationscoach möglich ist, bei Jugendlichen im Berufswahlprozess ihre schulischen Inhalte anzupassen. So kann es vorkommen, dass Jugendliche im Deutschunterricht die Möglichkeit haben, z.B. an Bewerbungen zu arbeiten. Die Option, die Jugendlichen beim Prozess ihrer Berufswahl und bei der Ausbildung selbst so eng begleiten zu können, scheint auch bei den Jugendlichen gut anzu- 
kommen. So sind Jugendliche enttäuscht, falls einmal ein Einzelcoaching ausfällt oder ein Besuch im Lehrbetrieb nicht stattfinden kann.

d) Mittlerweile besteht ein grosses Netzwerk aus der ehemaligen Schülerschaft. Oft kommen Ehemalige zu Besuch, berichten von ihrer Ausbildung oder halten einen Vortrag dazu - Peer Mentoring (vgl. Parsons \& Blake, 2004).

e) Ein weiterer, zentraler Punkt ist die Zusammenarbeit mit den Eltern. Speziell für die Eltern ist die Zukunft ihres Kindes oft eine grosse Sorge, was für den Berufsintegrationscoach gleichzeitig auch ein Anknüpfungspunkt für eine enge Zusammenarbeit mit den Eltern darstellt. Sorgen und Ängste können konstruktiv mit den Eltern besprochen und direkt in den Berufswahlprozess miteinbezogen werden.

\section{Zusammenfassung und abschließende Gedanken}

Zusammenfassend lässt sich sagen, dass die Einführung der sozialpädagogischen Angebote in Form der Stelle Sozialpädagogik und des Berufsintegrationscoachings die Qualität der Schule in Kleingruppen Dielsdorf gestärkt und weiterentwickelt hat. Denn die interdisziplinäre Zusammenarbeit begünstigt eine ganzheitlichere Erfassung von Schülerinnen und Schülern und ermöglicht es, den sozialen Entwicklungsschritten und Herausforderungen der Schüler und Schülerinnen mehr Gewicht zu verleihen. Zudem können diese zusammen mit unterschiedlichen Fachpersonen differenzierter bearbeitet werden.

Weiter lässt das Konzept der Schule in Kleingruppen zu, dass immer wieder Eins-zu-Eins-Situationen geschaffen werden können, was bei den Schülerinnen und Schülern gerne genutzt wird, um über Themen zu reden, welche sie beschäftigen, oder auch über Erlebnisse aus der Freizeit oder aus den Ferien zu berichten. Der Redebedarf, ausgedrückt als ,, Kann ich mal mit jemandem reden “, kann durch die Präsenz und das Angebotssetting der Sozialpädagogen an der Schule spontan ermöglicht werden.

Das Konzept der Schule in Kleingruppen bietet eine Vielzahl an Möglichkeiten, Beziehung zwischen Lernenden und Fachpersonen zu leben. Die gemeinsame Auseinandersetzung und die Teilhabe an den unterschiedlichen Lebenslagen und Problemen der Schülerinnen und Schüler sind beziehungsfördernd, was wiederum einen positiven Einfluss auf eine pädagogische $\mathrm{Zu}$ sammenarbeit und auch auf die Zusammenarbeit mit den Eltern hat.

Die Sozialpädagogik an Schulen bietet viele Zugänge und Möglichkeiten, Kinder und Jugendliche auf ihrem Weg in das Erwachsenenleben zu unterstützen. Obwohl sich in den meisten schweizerischen Regelschulen die 
Schulsozialarbeit mittlerweile bewähren konnte, scheint es in den Augen des Verfassers für die Angebote der Sozialpädagogik an schweizerischen Regelschulen noch viel mehr Entfaltungsmöglichkeiten zu geben, als bisher gewährt wurde. Gerade in einem für die jeweilige Schule angepassten Konzept eines Berufsintegrationscoachings würde viel Potential stecken. Die Schulsozialarbeit könnte durch das Berufsintegrationscoaching gestärkt und unterstützt werden und das Berufsintegrationscoaching könnte mit den bewährten Methoden Zugänge zu den Jugendlichen sichern und die Zusammenarbeit mit den jeweiligen Eltern begünstigen.

Die Verschränkung von Sozialpädagogik und Schulpädagogik in der Schule und die daraus resultierenden Angebote enthalten auch in Zukunft weiter auszuschöpfende Potentiale.

\section{Literatur}

Bildungsdirektion Kanton Zürich, Volksschulamt (2013). Rahmenkonzept Berufswahl und Lebensvorbereitung. „raschle \& partner“ (1. Aufl.). Bildungsdirektion Kanton Zürich.

Cassée, Kitty (2007). Kompetenzorientierung. Ein Praxisbuch mit Grundlagen, Instrumenten und Anwendungen. Bern, Stuttgart, Wien: Haupt Verlag.

Heckmaier, Bernd; Michl, Werner (1994). „Erleben und Lernen. Einstieg in die Erlebnispädagogik“, zweite Auflage. Neuwied, Kruchtel, Berlin. S. 66-67.

Hollenweger, Judith; Lienhard, Peter (2009). Schulische Standortgespräche. Ein Verfahren zur Förderplanung und Zuweisung von sonderpädagogischen Massnahmen. Lehrmittelverlag des Kantons Zürich. S. 18-22.

KGS: Schule in Kleingruppen (2017). www.kgs-dielsdorf.ch (26.08.2017).

Parsons, M.; Blake, S. (2004). Peer support: An overview, Spotlight, National Children's Bureau.

http://www.citized.info/pdf/external/ncb/Final_Peer_support_aw.pdf (25.10.2017).

Stangl, W. (2017). Stichwort: 'Case Management'. Online Lexikon für Psychologie und Pädagogik. www://lexikon.stangl.eu/13412/case-management/ (04.03.2018). 


\title{
Familienklassenzimmer
}

\author{
Markus Buholzer
}

An der öffentlichen Schule beobachten wir als Schulpsychologen, Heilpädagogen, Schulsozialarbeitende, Lehrpersonen und Schulleitung seit einigen Jahren, dass die Anzahl der Kinder stetig steigt, welche nicht die nötige Ausstattung an sozialer und emotionaler Reife mitbringen, um die verschiedenen Anforderungen, beispielsweise im Unterricht oder in den sozialen Kontakten, zu erfüllen. Die Ursachen sind vielschichtig und enden oft in einem unüberwindbaren Kreislauf von verhängnisvollen Verstrickungen. Auf störende Ereignisse im Umfeld reagieren betroffene Kinder mit Mängeln in ihrer Persönlichkeitsentwicklung. Findet in folgenden Interaktionen die Individualität des Kindes nicht im erforderlichen Umfang Beachtung, d.h., wird nur am Symptom „Kind“ und nicht auch am Familiensystem gearbeitet, treten eine Verschärfung und die Ausweitung der Gesamtproblematik ein. Der begonnene Kreislauf erfährt eine höhere negative qualitative und quantitative Form, was die Zunahme unangepasster Verhaltensweisen in Familie und Schule zur Folge hat (vgl. bspw. Schmid, 2007).

Zur erfolgreichen Bewältigung solcher sozialen Herausforderungen setzt die Volksschule Kriens (Primar- und Sekundarstufe) auf die Kooperation von Eltern und Schule (Disler, 2016b). Seit 5 Jahren bietet sie darum u.a. mit dem Eltern-Kind-Angebot „Familienklassenzimmer“ ein erfolgreiches schulisches Unterstützungsangebot mit bindungstheoretischem und multisystemischem Ansatz an (Volksschule Kriens, 2013; Disler, 2016b). Im folgenden Beitrag wird das Konzept „Familienklassenzimmer“ vorgestellt und reflektiert.

\section{Grundlagen des Familienklassenzimmers}

Das Familienklassenzimmer basiert auf drei zentralen bindungstheoretischen Konzepten: Mentalisierungsfähigkeit, Neue Autorität und Multifamilientherapie. Diese gründen auf dem systemischen Ansatz und werden im Folgenden skizziert. 
a) Die Förderung der sogenannten „Mentalisierungsfähigkeit" ist dabei ein zentraler methodischer Zugang. Hierbei geht es darum, die eigene innere Befindlichkeit, eigene Gefühle und diejenigen der Mitmenschen wahrzunehmen.

Schwierigkeiten sind nur in einer günstigeren Entwicklung aufzulösen, wenn die Schwierigkeiten als Beziehungs- und Kommunikationsstörungen von Menschen und von Institutionen, wie beispielsweise der Schule, begriffen werden. Daher kann erst ein nachhaltiger Beitrag zum gelingenden Aufwachsen des Kindes geleistet werden, wenn Familien, Eltern, Erziehungsbeauftragte, schulisches Personal und Kinder aufeinander zugehen und Herausforderungen zusammen angehen. Verschiedene Perspektiven und Blickpunkte abzugleichen und im Vergleich herauszufinden, welche Zugänge für eine Lösung besonders aufschlussreich sein können, wird als „systemisches Denken" bezeichnet (Journal für Schulentwicklung, 4/2011, S. 5). Aus meinem bald 20-jährigen Erfahrungshintergrund in der Schulführung geht klar hervor, dass die Schule heute die Aufgabe hat, vernetzt mit anderen Hilfssystemen und in Kooperation mit Eltern hinter die Verhaltensauffälligkeit eines Kindes oder Jugendlichen zu sehen und auf das Leiden oder auf eine andere verborgene Thematik eines Kindes oder Jugendlichen zu blicken (Preuss-Lausitz, 2013, S. 23).

Zudem sind entwicklungstraumatisierte Kinder in ihrem Grundvertrauen, ihrem Selbstwert, ihrer Selbstwirksamkeit und Beziehungsfähigkeit beeinträchtigt. Wenn sich das Kind sicher fühlt, wird das Erkundungssystem aktiviert und das Bindungssystem deaktiviert. Wenn sich das Kind unsicher fühlt, in Stress kommt, wird das Bindungssystem aktiviert und das Erkundungssystem deaktiviert (Brisch, 2015). Bindung vor Bildung ist Voraussetzung für neugierige Exploration und Lernen. Die Bezugspersonen sollten die innere Welt des Kindes verbalisieren und zusammen mit dem Kind regulieren, um herauszufinden, was den Stress verursacht.

b) Das Familienklassenzimmer basiert auf dem Ansatz der sogenannten „Neuen Autorität“ nach Haim Omer (2006). Es geht um die Frage, wie Menschen in ihren Systemen, wie beispielsweise der Schule oder der Familie, operieren. Omer geht es weniger um die Frage, wie die am Erziehungsprozess Beteiligten das Verhalten eines Kindes direkt verändern können, sondern wie sich ein Rahmen gestalten lässt, in welchem den Kindern und ihren Eltern neue Lernmöglichkeiten zur Verfügung gestellt werden. Somit ist unter „Neue Autorität“ das Lernsetting gemeint, das den Heranwachsenden und allen Beteiligten in Erziehung und Bildung bereitgestellt wird.

Das Konzept der „Neuen Autorität“ verfolgt folgende Ziele: 
- die Präsenz von Erwachsenen, welche jungen Menschen Halt und Orientierung gibt, zu stärken

- die Beziehung zwischen Lehrpersonen, Kindern und deren Eltern zu verbessern

- destruktives Verhalten von Kindern und Jugendlichen zu vermindern

- einen tragfähigen Rahmen für gelingende Erziehungsprozesse zu schaffen.

Der Ansatz der „Neuen Autorität" ist stark werteorientiert. Im Zentrum stehen Sicherheit, Beziehung, Entwicklung und Anerkennung. Der Bezug zu diesen Werten wird mit folgenden Elementen hergestellt (Omer \& von Schlippe, 2006).

- Die physische und zeitliche Präsenz drückt aus, dass ich da bin, weil mir die andere Person wichtig ist. Es geht um die Entscheidung, in gutem Kontakt mit mir selbst und respektvoll, wertschätzend und gewaltfrei der anderen Person gegenüber zu sein. Durch die Teilnahme im Familienklassenzimmer markieren Eltern Präsenz.

- Durch die Selbstkontrolle wird zum Ausdruck gegeben, dass man nur sich selbst, aber nicht andere kontrollieren kann. Erziehende können nur das eigene Verhalten bestimmen und dadurch dasjenige der Kinder beeinflussen. Das führt zu einer Stärkung der Erziehenden.

- Das Unterstützernetzwerk wird im Familienklassenzimmer aufgebaut. Das Wissen, nicht alleine zu sein und von anderen Eltern unterstützt zu werden, gibt Sicherheit, stärkt die Beziehungen und eröffnet neue Lösungswege.

- Anstatt unmittelbar auf destruktives Verhalten zu reagieren, teilen Erziehende mit, dass sie das gezeigte Verhalten des Kindes nicht tolerieren werden und dass sie nach einer Zeit darauf zurückkommen würden. Dadurch lassen sich Eskalationen und überstürzte Konsequenzen, die oft nicht umgesetzt werden können, vermeiden. In der gewonnenen Zeit können sich Erziehende vernetzen und Handlungsmöglichkeiten entwickeln, die den Aspekt einer Wiedergutmachung beinhalten.

- Schwierige Situationen werden transparent und damit öffentlich gemacht. Im Familienklassenzimmer kennen alle die Probleme der anderen. Dadurch wird die Sicherheit der Teilnehmenden erhöht.

c) Eine weitere Grundlage des Familienklassenzimmers ist die Multifamilientherapie nach Aia Asen (2009). Darunter versteht man eine kollegiale Beratung unter Familien mit der Idee, die Familien direkt in den Prozess ihrer eigenen Veränderungen einzubinden. Ziel der Multifamilientherapie im schulischen Kontext ist, in schwierigen Situationen (Leistungsschwierigkeiten, 
Disziplinarprobleme oder drohender Schulausschluss) die Ressourcen der Eltern und deren Kinder mit Unterstützung anderer Eltern und Kinder mit gleichartigen Problemen zu stärken. Schul- und Familienprobleme sollen gemeinsam angegangen werden. Die Leitenden der Familienklassenzimmer (vgl. Kapitel 2) handeln dabei als Begleitende oder Moderierende, indem sie die Problemlösungsfindung auf Basis eines systemischen Verständnisses fördern. Beispielsweise schaffen es eine Mutter und ihr Kind nicht, sich auf ein neues Teilziel mitsamt einer möglichen Umsetzung zu einigen. Anschreien, Vorwürfe und Erniedrigungen begleiten die Szene. Eine Bitte um Unterstützung der Leiterin des Familienklassenzimmers reicht, dass eine andere Mutter einschreitet, die beiden Frauen ihre Kinder „,auswechseln“, um die nächsten Entwicklungsschritte mit ihren nicht eigenen Kindern zu definieren. Die Situation beruhigt sich, die Offenheit für einen neuen Weg ist hergestellt.

Multifamilientherapie arbeitet mit der Erkenntnis, dass Schwierigkeiten keine isolierten Erfahrungen einzelner Familien sind und auch andere Menschen davon betroffen sind. „Wir sitzen alle im gleichen Boot" ist einer der bekanntesten Leitsätze der Multisystemtherapie. Die Anwesenheit von Familien mit ähnlichen Schwierigkeiten ist mit der Idee verbunden, dass sich Betroffene gegenseitig helfen, neue Lösungen zu finden und Ideen auszutauschen, um dadurch die Herausforderungen in der eigenen Familie anzugehen. Die Erfahrung, dass alle im selben Boot sitzen, soll zu mehr Akzeptanz des Problems und Selbstreflexion führen. Der Problemlösungsprozess wird dadurch angeregt, dass sich die Familien in anderen Familien ,gespiegelt“ fühlen und so neue Perspektiven eröffnet werden können. Wie Befunde aus der Evaluation des Familienklassenzimmers in der Volksschule Kriens zeigen, sieht man oft beim Gegenüber Dinge, die man selber bei sich nicht sieht (Disler, 2016a). So versucht eine Mutter dem Kind in einer Stuhlkreissituation mehrmals zu sagen, es solle aufhören sich so daneben zu benehmen und fordert es auf, sich endlich auf den Stuhl zu setzen wie alle anderen auch. Die anderen Familien im Kreis beobachten diese Situation. Automatisch stellt man sich die Frage: „Würde ich das auch so machen wie die andere Mama? Bin ich auch so hilflos? Ich mache das sicher nicht so, oder doch?"

Diese drei beschriebenen Ansätze bilden die Grundlage der Arbeit im Familienklassenzimmer und werden konsequent angewendet.

\section{Familienklassenzimmer in der Volksschule Kriens}

Im folgenden Abschnitt wird geschildert, wie das Konzept „Familienklassenzimmer" in der Volksschule Kriens umgesetzt wird. Dies geschieht anhand 
der Beschreibung der Strukturen und der Kriterien für den Eintritt ins Familienklassenzimmer, der Schilderung des Ablaufs und mit einigen Hinweisen auf die Evaluation.

\section{Struktur und Kriterien für den Eintritt ins Familienklassenzimmer}

Das Familienklassenzimmer wird von zwei Personen geleitet. Eine Person ist in sozialpädagogischer oder psychologischer Richtung ausgebildet, die andere Person ist eine Lehrperson der Volksschule Kriens. Eine der beiden Personen ist in Multifamilientherapie ausgebildet, beide haben die Grundlagen und Ansätze des Familienklassenzimmers verinnerlicht.

Eltern werden über die Lehrpersonen, die Schulsozialarbeitenden oder die Schulleitenden auf das freiwillige Angebot aufmerksam gemacht. In Eintrittsgesprächen, bei denen die Klassenlehrperson, die Schulleitung, die Leitung des Familienklassenzimmers und die Familie anwesend sind, wird beurteilt, ob das Familienklassenzimmer die richtige Massnahme für die entsprechende Fragestellung sein könnte. Andere Unterstützungsmöglichkeiten werden ebenfalls in Betracht gezogen. In diesen Eintrittsgesprächen entsteht ein klarer Eindruck über die Arbeitsweise, die Idee und die Chancen des Familienklassenzimmers. Das zu erreichende Hauptziel für das jeweilige Kind wird formuliert. Die Familien werden informiert, dass mit wöchentlichen Zielen gearbeitet und der Fokus auf den gemeinsamen Austausch des Elternteils und des Kindes gelegt wird. Das Eintrittsgespräch hilft vielen Eltern, die nötige Sicherheit zu erlangen, um der Teilnahme am Familienklassenzimmer zuzustimmen. Bedingungen werden transparent gemacht. So wird die Teilnahme davon abhängig gemacht, ob ein Elternteil während mindestens drei Monaten einen halben Tag pro Woche für das Familienklassenzimmer einsetzen kann. Viele Eltern unternehmen sehr viel, damit das möglich wird.

Für viele, aber nicht für alle Eltern, ist es möglich, sich für die Teilnahme am Familienklassenzimmer zu organisieren. Eltern mit noch jüngeren Kindern organisieren sich mit ihrem familiären Netzwerk, um die Kinderbetreuung zu garantieren. Erwerbstätige Eltern können manchmal auf kooperative Arbeitgeber zählen, um für den halben Tag zugunsten des Familienklassenzimmers frei zu nehmen. Ausserdem sind individuelle Lösungen möglich, indem sich die Elternpaare beispielsweise abwechseln oder ein älteres Geschwister den Elternteil (stundenweise) vertritt oder jüngere zu betreuende Geschwister ins Familienklassenzimmer mitgenommen werden. Dies ermöglicht die Übernahme von Verantwortung von verschiedenen Familienmitgliedern, was positive Auswirkungen auf die Beziehungsgestaltung innerhalb ihres Systems haben kann. Für viele Eltern ist der Besuch des Familienklassenzimmers eine zeitliche Belastung, die sie auf Grund einer schwierigen Situation und zum Wohle des Kindes annehmen. 
Nicht in allen, aber in den meisten Fällen nimmt die Mutter mit dem Kind am Familienklassenzimmer teil. Rund die Hälfte der Mütter ist alleinerziehend mit einem bis drei Kindern und steht dabei vor der Herausforderung, Beruf und Familie unter einen Hut zu bringen. Häufig können diese Mütter aufgrund der Doppelbelastung wenig Zeit für ihre Kinder aufbringen, was die Bindung zwischen Mutter und Kind beeinflusst. Die Familien mit Migrationshintergrund müssen sich - gerade in Bezug auf die Erziehung - mit den Werten, Normen und Anforderungen an die Eltern und Kinder auseinandersetzen. Sie verfügen teilweise über einen unsicheren Aufenthaltsstatus und ein kleines soziales Netz. Viele Familien sind ausserdem von finanziellen Unsicherheiten und/oder gesundheitlichen Belastungen betroffen. Einzelne Familien kennen keine der genannten Belastungen, sehen sich jedoch in der Erziehung des Kindes trotzdem mit grossen Herausforderungen konfrontiert.

Die Auseinandersetzung der Eltern mit ihren Erziehungsaufgaben und den damit verbundenen Herausforderungen gestaltet sich bei Eintritt ins Familienklassenzimmer sehr unterschiedlich; während sich einzelne Eltern bereits vorher im Rahmen von (Familien-)Coachings oder Eltern-/Erwachsenenbildung mit dem Thema Erziehung auseinandergesetzt haben, ist diese Art von Auseinandersetzung mit Erziehungsfragen für viele Eltern neu.

Die Kriterien für eine Vermittlung einer Familie an das Familienklassenzimmer sind sehr offen. Es geht um Schülerinnen und Schüler, die die Anforderungen beim Einhalten von Regeln und Arbeitsstrukturen nicht ausreichend erfüllen können. Die Kinder zeigen im Schulalltag herausforderndes Verhalten wie Verweigerung von Arbeitsstrukturen und Arbeitsaufträgen, Regelund Grenzverletzungen, Aggressivität, Respektlosigkeit gegenüber anderen Lernenden und Lehrpersonen, apathisches Verhalten, Isolation in der Klasse, Zurückgezogenheit und/oder häufige Abwesenheiten. Ein wichtiges Kriterium für die Teilnahme am Familienklassenzimmer ist, wenn die Beziehungsstärkung zwischen Eltern und Kindern ein zentrales Thema ist und alle Beteiligten - Eltern, Kinder und Lehrpersonen - gewillt sind, auf eine Veränderung hinzuarbeiten. Das Familienklassenzimmer wird teilweise als erstes Unterstützungsangebot herangezogen oder aber in Situationen, in welchen bereits viele Angebote ohne die erwünschte Wirkung in Anspruch genommen wurden. Eltern werden für das Familienklassenzimmer gewonnen, nicht verpflichtet.

Themen, welche auf Anregung der Schule zu einer Vermittlung ins Familienklassenzimmer führen, betreffen insbesondere das Verhalten der Heranwachsenden, Verweigerungen, das Befolgen von Regeln, Erfüllen von schulischen Aufgaben, die Leistungsbereitschaft oder Mobbingsituationen. Auch das Sozialverhalten im Kontakt mit Mitschülerinnen und Mitschülern und Lehrpersonen bzw. die Integration in der Klasse sind Thema. Bei einzel- 
nen Jugendlichen wurde das Familienklassenzimmer als Alternative zu einem schulischen Time-out oder einer Sonderbeschulung angesetzt, um innerhalb der schulischen Regelstrukturen ein Unterstützungsangebot zu ermöglichen. Teilweise wurde das Familienklassenzimmer auch als Übergangslösung genutzt, bis eine längerfristige Perspektive in Form eines begleiteten Wohnens oder einer externen Schule möglich war. Solche Übergangslösungen haben sich nicht bewährt und werden nicht mehr angeboten.

Die erlebten Belastungen zu Hause betreffen insbesondere die elterliche Präsenz und Wahrnehmung der Erziehungsaufgaben, die Kommunikation in der Eltern-Kind-Beziehung, Respekt der Kinder gegenüber ihren Eltern und das Befolgen der Regeln zu Hause (Essensregeln, Schlafzeiten, Freizeit, Mithilfe). Die betroffenen Kinder helfen im Haushalt wenig mit, respektieren Freizeitregeln kaum, verbringen viel Zeit mit digitalen Medien und die Hausaufgaben werden unzuverlässig erledigt. Durch die oftmals in den Familien herrschenden sozialen und finanziellen Belastungen (alleinerziehend, Belastung Familie-Beruf, Arbeitslosigkeit, Krankheit, Aufenthaltsstatus etc.) können die Eltern eingeschränkte Präsenz und Konsequenz zeigen und der Austausch zwischen Eltern und Kind kommt zu kurz. Eine alleinerziehende Mutter fühlt sich alleine mit ihrem Sohn und hat wenig Ideen, wie sie die Zeit mit ihm verbringen könnte. Einzelne Eltern mit Migrationshintergrund erwähnen, dass sie wenig wissen über die hiesigen Vorstellungen von Erziehung und sie daher ein Defizit wahrnehmen.

Andere geraten aufgrund des ausfälligen Verhaltens des Kindes zu Hause und in der Schule in Machtkämpfe und möchten im Familienklassenzimmer den Umgang damit üben. Bei diesen Familien wird das auffällige Verhalten des Kindes auch zur Belastung zu Hause.

\section{Ablauf des Familienklassenzimmers}

Die Volksschule Kriens bietet an zwei unterschiedlichen Halbtagen ein Familienklassenzimmer an, das von der Gemeinde finanziert wird. Die Grundstruktur ist in beiden Klassenzimmern gleich, in der Ausgestaltung und Gewichtung der einzelnen Phasen unterscheiden sie sich.

In der Eingangsrunde tauschen die Eltern und Kinder aus, was in der vergangenen Woche wichtig war und wie es ihnen aktuell geht. Es geht darum, die Gruppenkohärenz herzustellen.

Anschliessend wird eine angeleitete Körperarbeit mit Eltern und ihren Kindern durchgeführt, in welcher es darum geht, traumabedingte Spannungsund Stresszustände abzubauen. Für die Eltern und ihre Kinder sind dies einerseits neue Erfahrungen, die ihre Bindung stärken, anderseits erhalten sie ein Modell für weitere spielerische und kreative Begegnungen und Erlebnisse. 
In einer moderierten Sequenz werden Rückmeldungen über die Zielerreichung der vergangenen Woche zusammengetragen und besprochen. Dazu gehören auch Rückmeldungen der Klassenlehrperson, die über die gesetzten Ziele von den Leitenden des Familienklassenzimmers informiert wird. Erfolge und Misserfolge werden transparent gemacht, um gegenseitige Unterstützung zu ermöglichen. Die Aushandlung neuer Wochenziele rundet die Phase $\mathrm{ab}$.

Je nach Situation wird eine unterrichtsnahe Schulsequenz eingebaut, um die Schwierigkeiten der Kinder in ihrem Lernverhalten deutlich zu machen und um die Eltern in der Frage zu unterstützen, wie sie ihren Kindern adäquate Hilfestellungen geben können. Eltern erfahren, wie es ist, wenn ein Kind in einer Unterrichtssituation oft vom Arbeitsplatz davonläuft, nicht bei der Arbeit bleiben kann, sich dauernd von Kleinigkeiten ablenken lässt oder seinen Unmut laut kundtut. Elternhaus und Schule kommen sich hier nahe, es entsteht ein gemeinsamer kleiner Nenner. In der gemeinsamen Besprechung dieser Sequenz werden individuelle Unterstützungsmöglichkeiten besprochen. Die Erhöhung der Präsenz der Eltern gegenüber ihrem Kind ist auch in dieser Sequenz ein wichtiges Ziel.

Die Pause bietet Raum für den informellen Austausch unter den Eltern sowie offene Spielsequenzen für die Kinder.

Nach der Pause finden unterschiedliche Übungen, Spiele und Rollenspiele statt, mit dem Ziel, die Bindungsbeziehung, d.h. das Fürsorgeverhalten auf Seiten der Eltern (Bonding) und das Aufsuchen und Zulassen von Schutz und Unterstützung seitens der Kinder (Attachement), zu fördern.

Alle Sequenzen im Familienklassenzimmer sind darauf ausgerichtet, die inneren Befindlichkeiten des jeweils anderen wahrzunehmen, auszudrücken und verbalisieren zu können. Die Fähigkeit, das eigene Verhalten und das Verhalten anderer Menschen durch Zuschreibung mentaler Zustände zu interpretieren ist zentral. In einer Spielsituation nimmt ein Kind die Rolle eines Tieres ein, das aufgrund einer Notsituation gehegt und gepflegt werden muss. Das Tier leidet, hat Schmerzen und sucht die Nähe zur Mutter. Die Mutter kommt und kitzelt das Kind, um es zu erheitern. Solche Situationen werden mit der Gruppe besprochen, um der Mutter beratend zur Seite zu stehen und die Frage zu klären, welches Verhalten das Kind in seiner Rolle besser unterstützt hätte.

\section{Evaluation des Familienklassenzimmers}

Die Wirkung des Familienklassenzimmers wird von den Beteiligten an den wahrgenommenen Veränderungen festgemacht. Die Veränderungen werden vor allem an den verbesserten Eltern-Kind-Beziehungen, an einem besseren gegenseitigen Verständnis Eltern-Schule und an den angeeigneten Kompe- 
tenzen der Eltern festgestellt. Dies belegen Aussagen, die in den Austrittsgesprächen gemacht wurden:

- «Heute nach 19 Wochen im Familienklassenzimmer ist wieder Ruhe eingekehrt, unsere Beziehung ist entspannt, wir sind ruhiger geworden, alle haben ihren Platz gefunden und wir haben ein gutes Rüstzeug auf den Weg mitbekommen, um weiter zu machen.»

- «Früher habe ich immer gedacht, dass nur mein Sohn in der Schule Probleme macht und der Lehrer nur bei uns zu Hause anruft. Aber jetzt habe ich mich gut gefühlt. Ich habe gedacht, ah, andere haben auch Probleme.»

- «Durch das Beobachten der Kinder konnte ich besser verstehen, wo die Stärken und Schwächen meines Sohnes im schulischen Unterricht sind.»

- «Das Familienklassenzimmer ist ein grosses Geschenk, denn ich darf mein Kind einen ganzen Morgen begleiten und tolle Sachen mit ihm erleben, was sonst im Alltag zu kurz kommt.»

- «Das Familienklassenzimmer gibt Hoffnung und hilft nicht aufzugeben.»

- «Es ist einfacher Ziele zu erreichen.»

- «Mit der Zeit wird man bewusster, stärker, und zielorientierter.»

- «Der Meinungsaustausch ist wichtig - man sieht, dass man nicht alleine ist mit seinen Problemen. Es hilft die eigenen Stärken und Schwächen schneller zu entdecken.»

Einige Eltern nehmen sich stärker, kompetenter und konsequenter in ihrer Rolle als Erziehende wahr. Es gelingt ihnen besser, klarere Strukturen in den Familienalltag zu bringen. Sie bekommen Mut, etwas, was ihnen wichtig ist, durchzusetzen. Sie nehmen sich als Handelnde wahr, wenn sie „nein“ sagen und dabei bleiben können. Oft beschreiben Eltern, wie es ihnen gelingt, die Zeiten für die Benutzung der verschiedenen Medien zu regeln. Eine Mutter berichtet von der Erleichterung, nachdem ihr Sohn nach langer Zeit das erste Mal wieder in seinem eigenen Bett geschlafen hat. Andere Eltern stellen fest, einen anderen Umgang mit ihrem Kind gelernt zu haben, indem sie Kinder auf partizipative Weise einbeziehen, die Bedürfnisse der Kinder besser wahrnehmen und damit die Kinder besser begleiten können. Eltern schildern immer wieder, wie sich die Situation zu Hause entspannt, dass sie ihre Kinder besser verstehen und die Kinder zu Hause auch besser am Familienalltag teilnehmen, wie zum Beispiel ein Junge, der für seine Erfolge (der Mutter besser gehorchen, rechtzeitig ins Bett gehen und aufstehen, Hausaufgaben lösen, rechtzeitig nach Hause kommen, weniger essen) mit Kuschelzeit belohnt wird. Wieder andere Eltern geben an, ruhiger mit dem Kind zu kommunizieren, geduldiger mit ihm zu sein und seinen Bedürfnissen mehr Aufmerksamkeit zu schenken. 
Bei vielen Kindern verbessern sich das emotionale Befinden und ihr Verhalten deutlich, wie sie selbst berichten. Mehrere Kinder, die kurz vor einem Übertritt an eine externe Sonderschule standen, konnten in ihrer angestammten Klasse bleiben. Für viele Kinder konnte eine schwierige Situation so bearbeitet werden, dass sie sich wieder sicher fühlen. Allerdings braucht die Entwicklung Zeit. Die Familien sind meist bis zu einem Jahr im Familienklassenzimmer. Lehrpersonen wünschen sich oft, dass eine Verbesserung der Situation schneller eintritt. Je nach Notwendigkeit werden neben dem Familienklassezimmer noch andere Massnahmen beigezogen. Das kann eine noch intensivere und individuellere Begleitung der Familie in Form eines Familien- oder Elterncoachings sein oder eine Begleitung durch eine andere Fachstelle.

Das Familienklassenzimmer wurde von Februar 2014 bis Juni 2015 von der Fachhochschule Bern evaluiert (Erzinger \& Disler, 2015). Diese Evaluation beinhaltetet eine schriftliche Befragung aller Klassenlehrpersonen, aus deren Klasse ein Kind am Familienklassenzimmer teilgenommen hat, eine mündliche Befragung aller Eltern, eine schriftliche Befragung aller Kinder im Familienklassenzimmer, Beobachtungen im Familienklassenzimmer und Gruppengespräche mit den Leitenden des Familienklassenzimmers. In dieser Zeitspanne konnten 18 Eltern-Kind-Paare bei Eintritt und 15 Eltern und Kinder sowie 7 Klassenlehrpersonen bei Abschluss des Familienklassenzimmers (5 Familien) bzw. bei Abschluss der Evaluation (10 Familien) befragt werden. Ausserdem wurden an den zwei Familienklassenzimmer-Standorten insgesamt zehn Beobachtungen durchgeführt.

Die Empfehlungen gingen dahin, dass die unterschiedlichen Erwartungen, Anliegen, Ziele und Rollen aller Akteure transparenter und besser geklärt sein müssen. Insbesondere bei der Kommunikation und beim Austausch zwischen den Klassenlehrpersonen und dem Familienklassenzimmer wies die Evaluation Klärungsbedarf aus. Hinweise lieferte die Evaluation auch zur Rahmung des Familienklassenzimmers. In diesem Bereich wiesen die Evaluatoren darauf hin, dass die Überlegungen zu den Vorgehensweisen nachvollziehbarer gemacht werden sollen.

Die beiden Familienklassenzimmer wurden für die Primarschulkinder und deren Eltern entwickelt und aufgebaut. 2017 wurde eine erneute Standortbestimmung vorgenommen. Bei dieser Standortbestimmung wurden mündliche Interviews mit den Leitenden der Familienklassenzimmer und den Schulleitenden geführt. Die Erkenntnisse waren, dass die verschiedenen Strukturen und Abläufe immer noch geeignet sind, auch wenn sie aufgrund der mittlerweile mehrjährigen Erfahrung Anpassungen erfahren haben. So wurde beispielsweise mit der Zeit der Fokus bei den Arbeitsmethoden stärker auf das beziehungsfördernde Spiel gelegt. Weiter wurde bestätigt, dass das Familien- 
klassenzimmer in vielen Fällen eine positive Wirkung erzeugt und Veränderungen bewirkt. Wichtig und neu war die Erkenntnis, dass das Familienklassenzimmer auch schulintern immer wieder neu erklärt werden muss.

Aufgrund der positiven Erfahrungen wurde 2015 auch ein Familienklassenzimmer für die Jugendlichen der Sekundarschule aufgebaut. Die Standortbestimmung hat aber gezeigt, dass die Methodik des Familienklassenzimmers der Primarschule nicht auf die Jugendlichen angewendet werden kann, weil sie zu wenig auf deren Entwicklungsstand abgestimmt ist. Durch diese Erkenntnis wurde das Familienklassenzimmer der Sekundarschule durch andere Angebote ersetzt.

\section{Fazit}

Das Familienklassenzimmer stärkt die Eltern in ihren Erziehungsaufgaben und damit das Familiensystem. Dadurch fühlen sich Kinder und Eltern sicherer. Durch die erlangte Sicherheit kann das Kind sein Verhalten zu Hause und in der Schule anpassen. Das Kind muss seine Notsituation weniger oft oder nicht mehr mit auffallendem Verhalten zum Ausdruck bringen. Diese Veränderung tritt nicht sofort, aber nach einer gewissen Zeit ein, wie die Evaluationsbefunde belegen. Mehrere mögliche externe Sonderschulmassnahmen konnten verhindert oder verzögert werden. Herausfordernd sind Situationen, in denen Eltern der Teilnahme im Familienklassenzimmer zustimmen, in der Arbeit sich dann aber zu wenig öffnen und ihre Rolle im Familiensystem nicht erkennen können. In Situationen, in denen sich Eltern in ihren Familiensystemen nicht anders positionieren wollen oder können und dadurch der Rahmen für ihre Kinder nicht gestärkt wird, wirkt das Familienklassenzimmer wenig. Das Familienklassenzimmer kann nicht verfügt werden, die Eltern müssen den Nutzen selber erkennen. Abbrüche des Familienklassenzimmers gibt es keine oder nur in dem seltenen Fall, in dem die Eltern gemeinsam mit den Leitenden zu der Einsicht kommen, dass das Familienklassenzimmer nicht die richtige Massnahme ist.

Für die Schule ist und bleibt es eine Daueraufgabe, das systemische Denken und Handeln zu verankern. Die Volksschule Kriens bietet darum Informationsveranstaltungen und Weiterbildungen an, zunehmend auch für die Öffentlichkeit. Schwierige Situationen werden nach dem Modell der neuen Autorität und in Verbindung mit allen Systemen bearbeitet. Das erfolgreiche Bewältigen einer schwierigen Situation dank einer systemischen Vorgehensweise wird zunehmend als positiv erfahren. 


\section{Literatur}

Asen, E., Scholz, M. (2012). Praxis der Multifamilientherapie. Zweite vollst. überarbeitete und erweiterte Auflage. Heidelberg: Carl-Auer.

Baur. J. (2015). Selbststeuerung - Die Widerentdeckung des freien Willens. Dritte Auflage. München: Karl Blessing.

Brisch, Karl Heinz (2015). Kindergartenalter. München: Klett-Cotta

Disler, Stephanie (2016a). Familienklassenzimmer: Wo Kinder und Eltern gemeinsam Schule machen. BFH impuls 2/2016.

Disler, Stephanie (2016b). Eltern und Kinder gemeinsam im Familienklassenzimmer. knoten \& maschen. Blog des BFH-Zentrums Soziale Sicherheit. Verfügbar unter: https:/www.knoten-maschen.ch/tag/eltern/ [Zugriff: 04.03.2018].

Erzinger, Barbara, Disler Stephanie (2015). Familienklassenzimmer (FKZ) der Volksschule Kriens. Evaluationsbericht. Bern: Berner Fachhochschule. Verfügbar unter: https://www.volksschule-kriens.ch/public/upload/assets/1668/Evaluationsbericht _Familienklassenzimmer_Kriens_2015.pdf[Zugriff: 04.03.2018].

Omer, H., von Schlippe, A. (2006). Autorität durch Beziehung. Göttingen: Vandenhoek \& Ruprecht.

Omer, H., von Schlippe, A. (2010). Stärke statt Macht. Göttingen: Vandenhoek \& Ruprecht.

Preuss-Lausitz, U. (2013). Schwierige Kinder - schwierige Schule. Zweite erweiterte und aktualisierte Auflage. Weinheim und Basel: Beltz.

Schmid, Edeltraut (2007). Gestörte Beziehungen als Auslöser von Verhaltensauffälligkeiten und die Korrektur durch Halten und Halt geben bei älteren Kindern und Jugendlichen mit aggressivem Verhalten in der Schule. Verfügbar unter: http://www.adhs-schweiz.ch/ADHS_schule.htm [Zugriff: 04.03.2018].

Volksschule Kriens (2013). Familienklassenzimmer an der Volksschule Kriens. Kriens: Volksschule. 


\title{
Sozialpädagogik an der Volksschule Basel-Stadt - Schnittmengen und Stolpersteine
}

\author{
Marco Dalcher
}

\section{Die Schule als Lebensraum}

Die Schule als wichtiger Lebensort von Jugendlichen bietet sich im Sinne einer Lebensweltorientierung als Handlungsfeld der Sozialpädagogik an. Dabei ist es zentral zu erkennen, dass die Ziele der Schule mit den Zielen der Sozialpädagogik in vielen Punkten übereinstimmen: Die Stärkung der Persönlichkeit der jungen Menschen, die Förderung des eigenverantwortlichen Handelns oder die Qualifikation für das Leben in der Erwachsenenwelt sind Beispiele für solche gemeinsamen Ziele (vgl. EDBS, 2016). Beim Bestreben, bestmögliche Bedingungen für die erfolgreiche Bildung und Förderung junger Menschen in der Schule zu schaffen, entsteht so eine grosse Schnittmenge. Diese Schnittmenge gilt es als Handlungsfelder zu definieren, gegenseitig anzuerkennen und mit weiteren Angeboten der Schule (wie zum Beispiel die Freiwahlfächer oder die Begabungsförderung) zu koordinieren. Eine besonders bedeutende Rolle nimmt dabei die Kooperation ein. Einerseits mit den verschiedenen schulischen Partnern (z.B. Schulsozialarbeit oder Heilpädagogik) andererseits auch mit externen Partnern (z.B. aus der Jugendarbeit oder dem Sportamt) (bspw. Holtappels, Lossen, Spillebeen \& Tillmann, 2011). Ein Bewusstsein für unterschiedliche Aufgaben, Professionen und Betrachtungsweisen ist dabei ein wesentlicher Erfolgsfaktor. Ein fehlendes Ineinandergreifen der verschiedenen Angebote der Schule kann die Verfolgung der gemeinsamen Ziele an der Schule erschweren, was sowohl auf Erfahrungswerten beruht als auch aus der erwähnten Studie hervorgeht.

Unterrichtsergänzende Betreuung als sozialpädagogisches Handlungsfeld an der Schule

$\mathrm{Da}$ es die Schule genauso wenig wie die Sozialpädagogik gibt, erscheint es mir umso wichtiger, dass Unterschiede - ebenso wie Gemeinsamkeiten - der Schulpädagogik und der Sozialpädagogik (hier am Beispiel der Volksschule Basel-Stadt) diskutiert und dargelegt werden.

Die Tagesstrukturen, und mit ihnen die sozialpädagogische Betreuung der Schülerinnen und Schüler, haben sich als klassisches sozialpädagogisches An- 
gebot auf der Primarstufe der Volksschule Basel-Stadt in den letzten zehn Jahren etabliert. Die Fremdbetreuung von Kindern hat in der Schweiz eine lange Geschichte (vgl. hierzu den Beitrag von Staub in diesem Band). Wurden früher primär Kinder aus Familien im Hort betreut, deren Mütter aus wirtschaftlichen Gründen einer Erwerbsarbeit nachgehen mussten, so steht heute für viele Eltern der Wunsch nach Vereinbarkeit von Familie und Beruf im Zentrum. (EKFF, 2015). Bei der Entwicklung einer eigenständigen sozialpädagogischen Identität ist es aus meiner Sicht zentral, sich Klarheit über die aktuellen Aufgaben zu verschaffen, welche den Tagesstrukturen im Kontext aktueller gesellschaftlicher Rahmenbedingungen zugewiesen werden. Nur so kann sich die Tagesstruktur als eigenständiges sozialpädagogisches Arbeitsfeld etablieren. Ebenso zentral ist das Anerkennen, dass diese gesellschaftliche Aufgabe im Kontext der Schule übernommen wird. Die Einbettung in eine Institution des formalen Bildungsbereichs und ihren Einfluss auf die sozialpädagogische Handlungsweise der Tagesstrukturen gilt es zu benennen.

\section{Die Tagesstrukturen an der Sekundarschule des Kantons Basel-Stadt}

Während sich die Tagesstrukturen, und mit ihnen die sozialpädagogische Betreuung der Schülerinnen und Schüler an der Schule, auf der Primarstufe der Volksschule Basel-Stadt in den letzten zehn Jahren etabliert haben, sind sie auf der Sekundarstufe I für die Schule ein neues Angebot. Mit der Einführung der Sekundarschule im Jahre 2015 wurden gleichzeitig an allen zehn Sekundarschulstandorten der Volksschule Basel-Stadt auch Tagesstrukturen für Schülerinnen und Schüler eingesetzt. Dabei handelt es sich um ein unterrichtsergänzendes, freiwilliges Angebot, welches grundsätzlich aus einer kostenpflichtigen Verpflegung während der Mittagspause sowie einer beaufsichtigten Aufenthaltsmöglichkeit bis $17 \mathrm{Uhr}$ an der Schule besteht. Jedem Schulstandort ist es freigestellt, dieses Grundangebot mit weiteren Elementen (z.B. Freizeitkurse, Sportangebote oder Projekte) zu ergänzen. Es stellt sich sowohl die Frage nach der gewinnbringenden Ausgestaltung als auch nach der gelingenden Integration dieses Angebots in den Schulbetrieb.

Sozialpädagogische Grundhaltung der Tagesstrukturen an der Sekundarschule des Kantons Basel-Stadt

Der Aufbau der Tagesstrukturangebote an den Sekundarschulstandorten erfolgte durch die Tagesstrukturleitung (in anderen Kantonen als Leitung Betreuung bekannt) in enger Zusammenarbeit mit der Schulleitung. 
Während der Aufbauphase wurde deutlich, dass es für die Ausgestaltung der Angebote vor Ort pädagogischer Grundlagen bedarf, welche beispielsweise Hinweise zu sozialpädagogischen Handlungsfeldern oder zur sozialpädagogischen Grundhaltung der Tagesstrukturen geben. In der Folge wurde die Handreichung „Pädagogische Grundlagen für die Umsetzung der Tagesstrukturen an der Sekundarschule des Kantons Basel-Stadt“ (eduBS, 2017) erarbeitet. Darin wird die sozialpädagogische Grundhaltung der Tagesstrukturen wie folgt definiert:

\section{Die Tagesstruktur...}

- $\quad$ ist freiwillig, bedürfnisorientiert

- $\quad$ setzt auf die Ressourcen und auf das Potential der Jugendlichen

- fördert in hohem Masse die Eigenständigkeit der Jugendlichen

- ist partizipativ und prozessorientiert

- ist für alle Schülerinnen und Schüler zugänglich

- vermittelt Lebensfertigkeiten und leistet einen Beitrag zur Vorbereitung der Jugendlichen auf ihre Rolle als Erwachsene

- trägt zur Rhythmisierung des Ganztages der Jugendlichen an der Schule bei

- leistet einen Beitrag zum Wohlbefinden der Schülerinnen und Schüler im Lebensraum Schule

- erkennt Problemlagen von Jugendlichen und vermittelt diese an die Schulsozialarbeit

- leistet einen Beitrag zur Chancengerechtigkeit in der Bildung

Bei der konkreten Ausgestaltung der Angebote steht den einzelnen Standorten ein grosser Handlungsspielraum zur Verfügung. Dabei spielen die individuellen Unterschiede der Standorte eine bedeutende Rolle. Beispielsweise können Unterrichtskonzepte oder die Infrastruktur der Tagesstruktur unter den Schulstandorten stark kontrastieren. Ziel ist es, dass durch die standortspezifische Ausgestaltung der Tagesstruktur die Integration in den Schulbetrieb und die Akzeptanz der Tagesstruktur als schulisches Angebot vereinfacht wird. Dabei soll die Tagesstruktur aber immer als sozialpädagogisches Angebot erkennbar sein, indem sie sozialpädagogische Methoden anwendet, die im Unterschied zum Unterricht beispielsweise stärker auf die individuelle Förderung oder auf die selbstorganisierte Freizeitgestaltung fokussieren.

Sozialpädagogische Ausgestaltung der Angebote der Tagesstrukturen Die sozialpädagogische Ausgestaltung der Angebote der Tagesstrukturen kann sich, gemäss Handreichung „Pädagogische Grundlagen für die Umset- 
zung der Tagesstrukturen an der Sekundarschule des Kantons Basel-Stadt“ (2017), an folgenden sechs Kernpunkten orientieren.

\section{Ort des Vertrauens}

Im Jugendalter vollzieht sich der Übergang zum zukünftigen Erwachsenen. In dieser Phase ist die Suche nach der eigenen Identität eine zentrale Entwicklungsaufgabe (bspw. Hurrelmann \& Quenzel, 2016). In diesem Prozess kann es wiederholt zu Krisen kommen. Über eine vertrauensvolle Beziehung zu einer Ansprechperson zu verfügen, kann ein wesentliches, niederschwelliges Unterstützungselement in einer solchen Krise sein (ebd.). In der Tagesstruktur stehen solche Ansprechpersonen verlässlich zur Verfügung. Dabei können die Jugendlichen ohne Verpflichtung (Gesprächstermin, Gesprächssetting) die Ansprechperson und den Gesprächsinhalt spontan wählen.

\section{Raum für Begegnung und Beziehung}

Gleichaltrige Freunde (peers) werden oft als die besseren Kontakte und als hilfreicher bei der Bewältigung der Entwicklungsaufgaben angesehen als Erwachsene (ebd.). Ebenso oft sind sie die wichtigsten Bezugspersonen in dieser Phase (Chiapparini, 2017; Scherr, 2010). Um sich in der Peergroup auszutauschen, Beziehungen zu knüpfen und neue Verhaltensmuster auszuprobieren, braucht es Freiraum. Dieser offene Raum für Begegnungen und Beziehungen innerhalb der Peergroup bietet die Tagesstruktur den Jugendlichen an. Die eigenständige Nutzung und das Erkennen von Freiräumen werden so gefördert.

\section{Auseinandersetzung: Selbst- und Fremdbestimmung}

Die Beantwortung der Fragen „Wer bin ich?“ und „Wohin will ich in meinem Leben?" impliziert die Auseinandersetzung mit sich selbst (Mansel, 2003). Jugendliche können dabei leicht überfordert sein und sind auf Unterstützung von aussen angewiesen. Innerhalb der Tagesstrukturen wird beispielsweise das Verhalten der Jugendlichen reflektiert, es werden Konsequenzen daraus aufgezeigt oder es wird die Einhaltung von Regeln eingefordert.

\section{Eigenständigkeit und Verantwortung}

Dem Bedürfnis und der Anforderung nach wachsender Autonomie kann in der Tagesstruktur Rechnung getragen werden. Dies geschieht durch die Freiwilligkeit der Angebote der Tagesstruktur, mittels partizipativer Projekte, aber auch durch die selbstbestimmte Gestaltung der unterrichtsfreien Zeit in der Tagesstruktur. In Gesprächen über aktuelle Lebensthemen wird die Entwicklung von eigenständigen Bewältigungsstrategien gefördert. 


\section{Ort des Lernens}

Die Tagesstruktur versteht sich als Ort des Lernens. Einerseits wird mittels der Hausaufgabenunterstützung ein sehr konkreter Beitrag zum formalen Lernen an der Schule geleistet. Anderseits beinhaltet die Tagesstruktur viele Angebote aus dem non-formalen (z.B. Freizeit- oder Sportkurse) und informellen Bildungsbereich (z.B. Peergroup-Effekte, Verfolgung von individuellen Interessen).

\section{Erkennen und Fördern von Begabungen in den Tagesstrukturen}

Die Tagesstrukturen bieten ein grosses Potential hinsichtlich der Erkennung und Förderung von Begabungen, die abseits von schulischen Leistungsanforderungen liegen. So können Jugendliche beispielsweise je nach Angebot ihr motorisches Geschick, ihre kreativen Fähigkeiten oder ihre Führungskompetenzen beim Organisieren von Projekten (z.B. Filmprojekt) zeigen. Die Tagesstrukturen bieten Raum für die Verfolgung individueller Interessen, die im Unterricht möglicherweise keinen Platz haben. Es ist wesentlich, im Rahmen der Tagesstrukturen differenzierende Angebote zu schaffen, die die Jugendlichen auf unterschiedlichen Ebenen herausfordern und anregen. Durch die Vielfalt der Angebote können Jugendliche eigene Begabungen und Neigungen entdecken. Die Schaffung von Möglichkeiten zur Partizipation für Schülerinnen und Schüler im Tagesstrukturalltag ist dabei zentral.

\section{Chancen und Risiken}

Die Tagesstrukturen an der Sekundarschule Basel-Stadt befinden sich in einer Phase der Weiterentwicklung, denn die Angebotsgestaltung sowie deren Verankerung im Schultag ist ein laufender Prozess. Eine regelmässige Selbstevaluation unter Einbezug der Jugendlichen, der Mitarbeitenden und der Schulleitung ist angedacht. Dabei soll überprüft werden, ob die Angebote den Bedürfnissen der Jugendlichen entsprechen und das Wohlbefinden der Jugendlichen an der Schule fördern. Als Hilfsmittel dafür wurde ein Orientierungsraster als Evaluationsinstrument entwickelt (eduBS, 2017).

Durch die stetige Evaluation der Angebote kann der Entwicklungsprozess der Angebote gesteuert und bestehende Konzepte angepasst werden. Das Erkennen und die Reflexion von Stärken und Schwächen der Tagesstrukturen generell können für die Steuerung wesentliche Hinweise geben.

Basierend auf den bisher gemachten Erfahrungen lassen sich aus meiner Sicht folgende zentrale Stärken und Chancen sowie Schwächen und Risiken festmachen (vgl. auch Tab. 1). Dabei besteht kein Anspruch auf Vollständigkeit: 
Als eine wesentliche Stärke und gleichzeitig als Chance kann man sicher den Beitrag zur ganzheitlichen Bildung bezeichnen. Durch die verschiedenen persönlichkeitsbildenden Angebote der Tagesstrukturen wird die Identitätsentwicklung der Jugendlichen gefördert. Gleichzeitig übernehmen die Tagesstrukturen eine gesellschaftspolitische Aufgabe, indem sie einen wesentlichen Beitrag zur Vereinbarkeit von Familie und Beruf leisten. Der kontinuierliche Ausbau der Tagesstrukturplätze im Kanton Basel-Stadt während der letzten 10 Jahre belegt diesen grossen Bedarf eindrücklich.

Als Schwäche und gleichzeitig als Risiko lässt sich zum Beispiel die bisher noch fehlende Verankerung der sozialpädagogischen Arbeitsweisen an der Schule benennen. Bei der Integration von sozialpädagogischen Methoden in der Schule liegt aus meiner Sicht ein grosses Potential für die Weiterentwicklung der Schule als Lern- und Lebensort von Jugendlichen. Diese Integration muss meiner Meinung nach zwingend als gegenseitiger Prozess verstanden werden. Als ein weiteres Risiko kann die Abhängigkeit von politischen und gesellschaftlichen Entwicklungen bezeichnet werden. Dies könnte sich in Form von Einsparung von Ressourcen bei den Tagesstrukturen oder in einem Rückgang des Bedarfs an Tagesstrukturplätzen auswirken.

Tab. 1: Reflexionen, Chancen und Risiken von Tagesstrukturen mit Fokus auf die Sekundarschule

\begin{tabular}{|c|c|}
\hline Stärken & Chancen \\
\hline 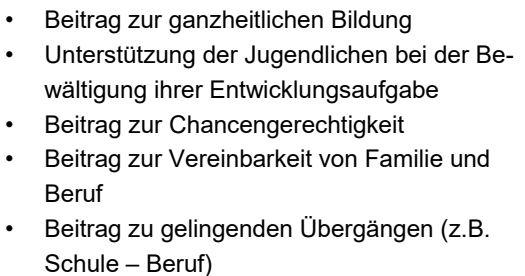 & $\begin{array}{l}\text { - Übernahme einer zugewiesenen gesell- } \\
\text { schaftspolitischen Aufgabe } \\
\text { - Steigender Bedarf an Ressourcen durch } \\
\text { grosse Nachfrage }\end{array}$ \\
\hline
\end{tabular}

\begin{tabular}{|l|l|}
\hline Schwächen & Risiken \\
\hline - Potential der Tagesstrukturen als sozialpä- & - Abhängigkeit von sozial-politischen Trends \\
dagogisches Handlungsfeld wird nicht er- & - Sozialpädagogisches Konzept wird von \\
kannt und nicht genutzt & $\begin{array}{l}\text { aussen nicht erkannt resp. anerkannt } \\
\text { - Fehlende Verankerung im Schulsystem }\end{array}$ \\
& Kaum dokumentierte Tradition der \\
& Sozialpädagogik an der Schule \\
& Ressourcen werden abgezogen \\
\hline
\end{tabular}

Quelle: eigene Zusammenstellung basierend auf Berufserfahrung in Basel-Stadt

Davon ausgehend, dass die Schule von vielen Jugendlichen nicht lediglich als Ort der formalen Bildung gesehen wird, sondern vielmehr einen wichtigen Aufenthaltsort darstellt, an dem sie oft den ganzen Tag mit Gleichaltrigen ver- 
bringen (Krüger, Deinert \& Zschach, 2012), kann man die Schule als eine bedeutende Lebenswelt der Jugendlichen betrachten (Grunwald \& Thiersch, 2016). Die Schule bietet einen wichtigen Entwicklungskontext an, in dem aktuelle Entwicklungsaufgaben bewältigt werden können. Im Übergang zum zukünftigen Erwachsenen ist die Suche nach der eigenen Identität eine dieser Entwicklungsaufgaben (Hurrelmann \& Quenzel, 2016). Das In-Frage-Stellen der Bezugspersonen, der eigenen Rolle in der Gruppe, die Entwicklung von romantischen Beziehungen sowie die Auseinandersetzung mit der beruflichen Zukunft können Anzeichen dieser Identitätssuche sein. Wie bereits erwähnt, nimmt die Peergroup dabei als Orientierung eine immer wichtigere Rolle ein und gewinnt an Tiefe. Das Ausprobieren von Selbstbestimmung und Selbstverwirklichung braucht Freiraum und Anerkennung der Eigenständigkeit. Diesen Freiraum kann die Tagesstruktur innerhalb der Lebenswelt Schule bieten, indem geleitete und selbstorganisierte Freizeitgestaltung während der Mittagspause und nach dem obligatorischen Unterricht stattfindet. Damit wird ein Lernsetting geschaffen, das weniger geleitet und schulleistungsorientiert ist, sondern vielmehr auf Selbstorganisation, Persönlichkeitsbildung und auf die Förderung von Sozial- und Selbstkompetenz fokussiert. Gleichzeitig stehen erwachsene Ansprechpersonen zur Verfügung, welche keine beurteilenden Aufgaben im Rahmen des obligatorischen Unterrichts an der Schule übernehmen. Dies kann Jugendlichen (z.B. bei der Bewältigung von Stress oder Angst) Sicherheit verleihen. Als ebenso wichtig wird die Tagesstruktur als Ort der Erholung bezeichnet (unveröffentlichte Befragung der Besucherinnen und Besucher von Tagesstrukturen der Sekundarschule Basel-Stadt, Woche 36, 2017). Auf die Frage „Was machst Du in der Tagesstruktur am liebsten?“ war die Antwort „chillen“ mit Abstand die häufigste. Das Bedürfnis nach Erholung scheint derart gross zu sein, dass von einer hohen Belastung der Schülerinnen und Schüler im Schulalltag ausgegangen werden kann. Vor diesem Hintergrund erscheint das Angebot eines Ortes an der Schule, an dem man sich zurückziehen und erholen kann, als durchaus legitim. Ebenfalls geschätzt - wenn auch deutlich weniger - werden Angebote wie: Sport treiben, Spielen oder Unterstützung bei der Erledigung von Hausaufgaben.

\section{Fazit}

Aus den vorausgegangenen Ausführungen geht hervor, dass die Schule für Jugendliche und für die Peergroup als Ort der Entwicklung, der Persönlichkeitsbildung, der Freundschaften mit Gleichaltrigen und der Erholung betrachtet werden kann. Diese Perspektive auf die Schule ergänzt das unterrichtliche Lernen. Deshalb stellt sich die Frage, was man (je nach Arbeitsbe- 
reich) unter „Förderung“ versteht. Im Unterricht und in der Tagesstruktur kann sich dieses Verständnis durchaus unterscheiden (Leistungsförderung oder Persönlichkeitsbildung). Wichtig erscheint mir, dass dieser Unterschied als Ergänzung und als Erweiterung des Lernangebots an der Schule betrachtet wird. Wird Lernen an der Schule als ganzheitlicher Lernprozess verstanden, eröffnet sich für die Sozialpädagogik ein weites Betätigungsfeld. Voraussetzung dafür ist allerdings, dass die Schule als Ort gesehen wird, an dem neben dem Bildungsauftrag (im Sinne der formalen Bildung) der Erziehungsauftrag (im Sinne der Persönlichkeitsbildung) ebenso anerkannt wird. Für die Schule mit ihren stabilen Strukturen und ihrer festen Tradition bedeutet dies, sich einer anderen Profession mit einem zum Teil anderen Verständnis von Bildung zu öffnen. Umgekehrt gilt es für die Sozialpädagogik, sich im Kontext der Schule zu definieren und sich weiterzuentwickeln. Dabei ist eine gegenseitige Akzeptanz der Handlungsfelder aus meiner Sicht lediglich ein erster Schritt.

Idealerweise entwickeln sich die beiden Handlungsfelder von einem Nebeneinander hin zu einem Miteinander. So können die professionellen Stärken beider Berufsgruppen sich gegenseitig ergänzend genutzt und Synergien zum Wohl des Kindes (bzw. Jugendlichen) eingesetzt werden.

Die scharfe Aufteilung zwischen Schulpädagogik und Sozialpädagogik sowie die damit verbundene Abgrenzung der beiden Berufsgruppen würde so überwunden und verabschiedet. In der Folge böten sich vielfältige Möglichkeiten, den Ganztag an der Schule neu zu denken und zu gestalten - stets mit dem Wohlbefinden und der ganzheitlichen Förderung der Schülerinnen und Schüler im Fokus.

\section{Literatur}

Chiapparini, E. (2017). Schule und Freizeit. Partizipative Angebote für Kinder und Jugendliche. punktum. (5), 12-14.

Eidgenössische Koordinationskommission für Familienfragen (EKFF) (2015). Schulergänzende Betreuung aus Eltern- und Kindersicht. Unter Mitarbeit von Stephanie Schwab Cammarano, Susanne Stern, Donald Sigrist, Infras. Bern: BBL.

Erziehungsdepartement Basel-Stadt (eduBS) (2017). Pädagogische Grundlagen für die Umsetzung der Tagesstrukturen an der Sekundarschule des Kantons Basel-Stadt.

Erziehungsdepartement Basel-Stadt (eduBS) (2017). Tagesstrukturen an der Sekundarschule. Orientierungsraster für die Schulentwicklung und Schulevaluation an den Volksschulen des Kantons Basel-Stadt. Retrieved from https://www.edubs.ch/schulentwicklung/link/downloads/Bro_BS_Tagesstrukture nSek_v04.pdf/at_download/file [Zugriff: 07.03.2018].

Erziehungsdepartement des Kantons Basel-Stadt (EDBS) - Volksschulen (2016). Lehrplan 21. Stand 29.02.2016 (Gesamtausgabe). Verfügbar unter http://bs.lehrplan.ch/container/BS_DE_Gesamtausgabe.pdf [Zugriff: 07.03.2018]. 
Grunwald, K. \& Thiersch, H. (Hrsg.) (2016). Praxishandbuch Lebensweltorientierte Soziale Arbeit: Handlungszusammenhänge und Methoden in unterschiedlichen Arbeitsfeldern (3., vollständig überarbeitete Auflage). Grundlagentexte Pädagogik. Weinheim: Beltz Juventa.

Holtappels, H.-G., Lossen, K., Spillebeen, L. \& Tillmann, K. (2011). Schulentwicklung und Lehrerkooperation in Ganztagsschulen. Konzeption und Entwicklungsprozess als förderliche Faktoren der Kooperationsentwicklung? In N. Fischer, H.G. Holtappels, E. Klieme, T. Rauschenbach, L. Stecher \& I. Züchner (Eds.), Ganztagsschule: Entwicklung, Qualität, Wirkungen. Längsschnittliche Befunde der Studie zur Entwicklung von Ganztagsschulen (StEG) (pp. 25-42). Weinheim: Juventa.

Hurrelmann, K. \& Quenzel, G. (2016). Lebensphase Jugend: Eine Einführung in die sozialwissenschaftliche Jugendforschung (13., überarbeitete Auflage). Grundlagentexte Soziologie. Weinheim: Beltz Juventa.

Krüger, H.-H., Deinert, A. \& Zschach, M. (Hrsg.) (2012). Jugendliche und ihre Peers: Freundschaftsbeziehungen und Bildungsbiografien in einer Längsschnittperspektive. Opladen: Verlag Barbara Budrich.

Mansel, J. H. K. (2003). Jugendforschung und Sozialisationstheorie. Über die Möglichkeiten und Grenzen der Lebensgestaltung im Jugendalter. In J. Mansel, H. M. Griese \& A. Scherr (Eds.), Jugendforschung. Theoriedefizite der Jugendforschung: Standortbestimmung und Perspektiven (pp. 75-90). Weinheim: JuventaVerl.

Scherr, A. (2010). Cliquen/informelle Gruppen: Strukturmerkmale, Funktionen und Potentiale. In M. Harring, O. Böhm-Kasper, C. Rohlfs \& C. Palentien (Eds.), Freundschaften, Cliquen und Jugendkulturen. Peers als Bildungs- und Sozialisationsinstanzen (pp. 73-90). Wiesbaden: VS Verlag für Sozialwissenschaften. 


\title{
„Auch wir haben was zu sagen!“ - Beitrag zur Qualitätssteigerung in Tagesschulen durch Kooperation in interprofessionellen Teams
}

\author{
Simon Benz
}

Innerhalb der theoretischen wie praxisrelevanten Auseinandersetzung mit Tagesschulen ${ }^{1}$ in der Schweiz rückt die Thematik um „Kooperation in interprofessionellen Teams" vermehrt in den Fokus der beteiligten Profession. Dabei wird Kooperation als relevantes Qualitätsmerkmal eingestuft und in diversen Konzepten gesondert berücksichtigt sowie für die Praxis aufbereitet $^{2}$. Speck, Olk, Böhm-Kasper, Stolz, Wiezorek (2011, S. 11-12) meinen da$\mathrm{zu}$ :

Diese Entwicklung ist unabdingbar, da Kooperation zwischen Lehrpersonen mit inner- sowie ausserschulischen Partnerinnen und Partnern an Tagesschulen auch darauf ausgelegt sein sollte, Kinder und Jugendliche umfassender zu fördern, schulische und soziale Benachteiligung zu vermeiden, non-formale Bildungs- und Betreuungsangebote sicherzustellen, Übergänge von Kindern und Jugendlichen in weiterführende Schulstufen zu optimieren und neue Bildungsorte und Gelegenheiten zu erschliessen.

Dadurch rücken Fachpersonen der Sozialen Arbeit vermehrt in den Fokus solcher Kooperationsprozesse.

Im ersten Teil dieses Beitrages geht es um die Auseinandersetzung mit aktuellen Forschungserkenntnissen bezüglich Kooperation in multiprofessionellen Teams. Dabei wird der Fokus auf die Entwicklung einer Schulkultur

1 Unter einer Tagesschule oder Tagesstrukturen versteht man in der Schweiz überwiegend eine schulische Institution mit einem Angebot, welches den ganzen Schultag abdeckt. An einer Tagesschule gibt es teilweise einen unterrichtlichen und einen ausserunterrichtlichen Teil oder aber die beiden Teile verschmelzen im Schultag (Schüpbach, 2010, S. 13). Tagesschule sowie Tagesstruktur sind gleichzusetzten mit dem in Deutschland geläufigen Begriff der Ganztagsschule. Die beiden Begriffe werden im vorliegenden Beitrag synonym verwendet.

2 Die Publikation „Qualität in Tagesschulen/Tagesstrukturen (QuinTaS)“ liefert einen Qualitätsrahmen, bestehend aus einem Grundlagenbuch und sechs Arbeitsbüchern, mit Hilfe dessen Qualitätsmerkmale überprüft und implementiert werden können. Für diesen Beitrag ist insbesondere das Arbeitsbuch 3 „Kooperation“ (Kuster \& Bussmann, 2017) zu berücksichtigen. 
gelegt, welche Kooperation mit einem starken Partizipationsgedanken verknüpft. Für die Praxis bedeutet dies, dass die Fachpersonen der Sozialen Arbeit vermehrt in die Entwicklung der Schulkultur einbezogen werden sollten, damit diese partizipative Einbindung zu einer professionsübergreifenden und intensiveren Kooperation innerhalb interprofessioneller Teams führen kann. Die gewonnenen Erkenntnisse werden weiterführend in einen praxisnahen Reflexionenrahmen eingebunden, indem Referenz auf ein konkretes Konzept einer bestehenden Tagesschule der Stadt Zürich genommen wird. Diese Gegenüberstellung liefert das Fundament für die Auseinandersetzung mit der Fragestellung, inwiefern Fachpersonen Sozialer Arbeit als Teil des Betreuungsbereichs in multiprofessionellen Kooperationsprozessen in Tagesschulen einen Beitrag zur Qualität leisten können.

\section{Kooperation innerhalb interprofessioneller Teams}

„In der Fachdiskussion besteht weitgehend Konsens in der Annahme, dass sich die Umsetzung der Tagesschule nur mit der Vernetzung der Ressourcen und Kompetenzen anderer, an Tagesschulen relevanter Berufsgruppen ${ }^{3}$ bewerkstelligen lässt" (Speck, Olk, \& Stimpel, 2011, S. 69). Tillmann und Rollett (2011, S. 33) haben bezugnehmend auf diese Auffassung zwei für die Kooperationsforschung relevante aufeinanderfolgende Aspekte herausgearbeitet. Einerseits gehen sie aufgrund von Befunden zu Effekten der Lehrerpartizipation auf die Lehrerkooperation davon aus, dass eine intensivere Partizipation der Fachpersonen der Sozialen Arbeit die Kooperation mit Lehrpersonen stützt. Andererseits kann unter Berücksichtigung dieser Argumentationslinie zusätzlich davon ausgegangen werden, dass eine ausgeprägte strukturelle Einbindung der Fachpersonen der Sozialen Arbeit die Kooperationskultur innerhalb des Schulbetriebs verbessert.

Da zudem empirisch nachgewiesen wurde, dass sich Kooperationsintensität und strukturelle Einbindung gegenseitig unterstützen und verstärken (Fischer et al., 2011), bietet sich die Implementation von Massnahmen ${ }^{4}$ an, welche unter anderem die Intensität fördern, als auch solcher, welche die Erwei-

3 Unter der Subsumierung, ,relevante Berufsgruppen“werden im vorliegenden Beitrag in erster Linie die im Schulkontext tätigen Fachpersonen der Sozialen Arbeit gefasst. Weiterführend muss in diesem Zusammenhang jedoch auch von Berufen wie Fachfrau/Fachmann Betreuung, den im Betreuungsbereich tätigen betrieblichen Assistenten oder den sonderschulpädagogischen Fachlehrpersonen gesprochen werden.

4 Weitere Erkenntnisse betreffend Massnahmen zur Kooperation in Tagesschulen liefert die qualitative Studie von Schüpbach, Jutzi und Thomann (2012). 
terung der Partizipation für die Fachpersonen der Sozialen Arbeit ${ }^{5}$ unterstützen, sich aktiv an der Planung, Entwicklung und Gestaltung des Tagesschullebens zu beteiligen (Tillmann \& Rollett, 2011, S. 44).

Bezugnehmend auf die oben referierten Befunde wird nachfolgend Partizipation als kooperationsverstärkender Mechanismus genauer betrachtet. Tillmann und Rollett (2011, S. 35) liefern dazu eine differenzierte Definition, welche in einem Index für Partizipation von Fachpersonen der Sozialen Arbeit $^{6}$ folgendermassen zusammengefasst wird: „Partizipation kann sich auf die Mitarbeit in Gremien und Steuergruppen, die Entwicklung des Tagesschulkonzepts, die Organisation und das Management des laufenden Tagesschulbetriebs sowie die Vorbereitung und Durchführung schulischer Veranstaltungen beziehen“. Somit steht das Verständnis von Partizipation in der Tradition der Partizipationsleiter nach Hart (1997), welche sich an den letzten drei Stufen, namentlich der Mitbestimmung, Selbstbestimmung und Selbstverwaltung, orientieren sollten.

\section{Einordnung der empirischen Erkenntnisse in der Praxis}

Aufbauend auf die oben skizzierten Erkenntnisse wird nachfolgend exemplarisch an einem bestehenden Betreuungskonzept einer Tagesschule ${ }^{7}$ aufgezeigt, wie sich intensivere Kooperation zwischen Fachpersonen der Sozialen Arbeit und Lehrpersonen in der Praxis gestalten lässt.

Die besagte Tagesschule kann auf eine lange Tradition von Kooperation in interprofessionellen Teams zurückblicken. Entsprechend haben sich strukturelle Rahmenbedingungen etabliert, welche mit den empirischen Erkenntnissen von Tillmann und Rollett übereinstimmen. Daher bietet sich eine exemplarische Darstellung unter Einbezug persönlicher Erfahrungswerte und einer anschliessenden konzeptorientierten Reflexion an.

5 Tillmann und Rollett sprechen im Original vom ,weiteren pädagogisch tätigen Personal“. Aus Positionierungsgründen wird in diesem Beitrag konsequent von Fachpersonen der Sozialen Arbeit gesprochen.

6 Vgl. Fussnote 7.

7 Die Tagesschule wurde 1992 als eine von sieben Pilottagesschulen der Stadt Zürich per Gemeinderatsbeschluss in der Volksschule verankert. Der Unterschied zu einer Schule mit integriertem Hort war seit Beginn die intensive Entwicklung von Kooperationsmechanismen zwischen Lehr- und der Fachpersonen Sozialer Arbeit. 
Tab. 1: Kooperationsprozesse zwischen Betreuung und Lehrpersonen (Quelle: Eigene Darstellung, basierend auf dem unveröffentlichten Betreuungskonzept Tagesschule)

\begin{tabular}{|c|c|}
\hline Kooperationsgremien & Ausgestaltung \\
\hline Stufenteam & 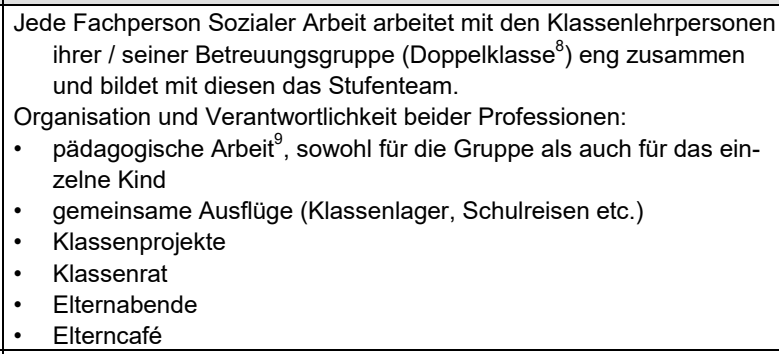 \\
\hline Sitzungen & $\begin{array}{l}\text { Die Fachpersonen der Sozialen Arbeit vertreten in allen schulischen } \\
\text { Gremien die Anliegen des Betreuungsbereichs gemäss dem Berufsauf- } \\
\text { trag und arbeiten an schulinternen Entwicklungsprozessen mit. } \\
\text { Die Teilnahme an den } 14 \text { jährlich stattfindenden Tagesschulsitzungen } \\
\text { ist für Fachpersonen der Sozialen Arbeit obligatorisch (ab } 40 \text { Stellen- } \\
\text { prozent). } \\
\text { Ebenso ist die Teilnahme an den Sitzungen der pädagogischen } \\
\text { Teams }{ }^{10} \text { alle zwei Wochen obligatorisch, welche in die Bereiche Unter- } \\
\text { stufe und Mittelstufe unterteilt sind (ab } 40 \text { Stellenprozent). }\end{array}$ \\
\hline Schulkonferenz & $\begin{array}{l}\text { Die Teilnahme an der Schulkonferenz, in der Regel einmal pro Monat, } \\
\text { ist für Fachpersonen der Sozialen Arbeit ab einem Stellenpensum von } \\
40 \% \text { obligatorisch. }\end{array}$ \\
\hline Q-Gruppen & $\begin{array}{l}\text { Alle Fachpersonen der Sozialen Arbeit sind gemäss Jahresplanung der } \\
\text { Schuleinheit Mitglied einer Q-Gruppe. }{ }^{11}\end{array}$ \\
\hline $\begin{array}{l}\text { Zusammenarbeit mit der } \\
\text { Schulleitung }\end{array}$ & $\begin{array}{l}\text { Die Schulleitung nimmt an der wöchentlich stattfindenden Betreu- } \\
\text { ungsteamsitzung teil. } \\
\text { Eine Fachperson der Sozialen Arbeit übernimmt die Funktion der An- } \\
\text { sprechperson für die Schulleitung. }\end{array}$ \\
\hline Informelle Gespräche & $\begin{array}{l}\text { Die Fachpersonen der Sozialen Arbeit bemühen sich um den informel- } \\
\text { len Austausch innerhalb des Schulteams. Sie besuchen z.B., wenn } \\
\text { möglich, die 10-Uhr-Pause und suchen den aktiven Austausch mit den } \\
\text { Lehrpersonen. }\end{array}$ \\
\hline
\end{tabular}

8 Unter dem Begriff Doppelklasse werden altersdurchmischte Klassen verstanden.

9 Die pädagogische Arbeit setzt sich aus den Besprechungen der verschiedenen Gremien (Pädagogische Teamsitzung, Stufenteam, Schulkonferenz, Q-Gruppe, Betreuungsteamsitzung), der Orientierung am pädagogischen Konzept, dem Berufsauftrag sowie dem persönlichen Fach- und Erfahrungswissen zusammen.

10 Pädagogische Teams setzen sich aus den Lehrpersonen und Fachpersonen der Sozialen Arbeit aus dem Unter- und Mittelstufenbereich zusammen, welche getrennt voneinander an bereichsspezifischen sowie -übergreifenden Themen arbeiten.

11 Die interne Schulentwicklung wird in der Stadt Zürich in Qualitätsgruppen (Q-Gruppen) vollzogen. 
Bei genauerer Betrachtung der vorangestellten Tabelle fällt auf, dass die strukturelle Rahmung von Stufenteams bis hin zum vollständigen Team einer Einbettung folgt, welche vorwiegend durch formelle Austauschsituationen geprägt ist. Dabei scheint es unumgänglich, auf die notwendige und steuernde Wirkung funktionierender Konzepte hinzuweisen, in denen das inhaltliche wie professionelle Profil der Sozialen Arbeit erkennbar ist (Thimm, 2015, S. $34)$.

Die Kooperation im präsentierten Beispiel findet auf verschiedenen organisatorischen und strukturellen Ebenen statt und basiert auf dem Grundsatz, dass die Bereiche Schule und Betreuung nicht getrennt voneinander betrachtet werden. Hierfür ist eine gelingende Kooperation innerhalb der Stufenteams von besonderer Relevanz. Eines der zentralen Ziele dieser Stufenteams ist die Entwicklung von Wertesystemen, welche gemeinsam im Betreuungs- sowie Schulbereich gelebt werden. Zur Implementierung dieser Wertesysteme bieten sich Gremien wie der Klassenrat, Ausflüge, Projekte, Mittagszeiten ${ }^{12}$ oder das Elterncafé an, da diese gemeinsam gestaltet und durchgeführt werden. Dadurch entsteht eine differenziertere und ganzheitliche Betrachtung und Bewertung von schulinternen Themen, welche direkt oder indirekt auf weitere Kooperationsebenen einwirken. In der Praxis zeigt sich dies beispielsweise dadurch, dass es für eine Lehrperson der zuvor erwähnten Tagesschule selbstverständlich ist, Fachpersonen der Sozialen Arbeit bei Situationen oder Konflikten einzubeziehen, um das weitere Vorgehen im Stufenteam zu besprechen.

Zusätzlich muss der informelle Bereich mit spezifischen Kriterien konkretisiert und intensiviert werden, da ansonsten das Potential der informellen und personalisierten Kooperationsformen nicht ausreichend genutzt werden kann. Laut Horstkemper (2011, S. 134ff.) legen empirische Befunde diesbezüglich eine Investition in informelle Kooperationsprozesse nahe, da diese im Vergleich zu den stark formalisierten Verfahren eine positivere Wirkung zu haben scheinen.

Tabelle 2 stellt einen auf Kooperationsmechanismen begrenzten Auszug eines Hortkonzeptes dar, welcher implizit eine Reihe von möglichen Polaritäten innerhalb einer gelebten Interprofessionalität enthält. Im Wesentlichen geht es dabei um Akzeptanz-, Rollen- und Kooperationsprobleme, welche durch verschiedene konzeptionelle sowie reflexive Überlegungen entflechtet und nachfolgend in Form einer Tabelle dargestellt werden.

12 Lehrpersonen arbeiten bis zu zwei Tage in der Woche über Mittag in der ausserunterrichtlichen Betreuung. 


\section{Tab. 2: Interprofessionelle Problemkategorien und ihre konzeptuelle Auflö-}

sung (Quelle: Eigene Darstellung)

\begin{tabular}{|c|c|}
\hline Problemstellung & $\begin{array}{l}\text { Darlegung und konzeptionelle Überlegung zur Auflösung nachgestellter } \\
\text { Probleme }\end{array}$ \\
\hline & $\begin{array}{l}\text { Darlegung des Problems } \\
\text { „Akzeptanzprobleme entstehen häufig dann, wenn Sozialarbeitende nicht in die } \\
\text { Kernzonen der Schule mit eigenständigen Positionen integriert sind“ (Thimm, } \\
\text { 2015, S. 34). }\end{array}$ \\
\hline $\begin{array}{l}\text { Akzeptanz- } \\
\text { probleme }\end{array}$ & $\begin{array}{l}\text { Konzeptorientierte Reflexion } \\
\text { Durch die konzeptionelle Festschreibung, dass der Betreuungsbereich in allen } \\
\text { Austauschsitzungen eine auf dem Berufsauftrag basierende Perspektive ein- } \\
\text { nehmen und mit Hilfe derer die Schulentwicklung vorantreiben soll, wird eine kla- } \\
\text { re Integration mit eigenständigen, die Profession betreffenden Positionen gefor- } \\
\text { dert und gefördert. Diese konzeptionelle Steuerung führt unweigerlich zu einem } \\
\text { Diskurs, der die unterschiedlichen Deutungen bezüglich der Bedürfnisse und An- } \\
\text { sprüche der Adressatengruppen der Tagesschule und der damit einhergehenden } \\
\text { pädagogischen Herausforderungen betrifft. Weiterführend kann diese Debatte in } \\
\text { den bestehenden Gremien diskutiert werden. Dabei muss die Integration eigen- } \\
\text { ständiger Positionen durch die Fachpersonen der Sozialen Arbeit forciert und } \\
\text { eingefordert werden. }\end{array}$ \\
\hline \multirow[b]{2}{*}{$\begin{array}{l}\text { Rollen- } \\
\text { probleme }\end{array}$} & $\begin{array}{l}\text { Darlegung des Problems } \\
\text { „Oftmals sind die Rollen nicht genügend geklärt bzw. kann Rollenunschärfe die } \\
\text { Beteiligten verunsichern“ (Thimm, 2015, S. 35). }\end{array}$ \\
\hline & $\begin{array}{l}\text { Konzeptorientierte Reflexion } \\
\text { Die Zusammenarbeit in Stufenteams fordert eine präzise Rollenklärung, um die } \\
\text { gemeinsamen Aufgaben und Ziele zu erreichen. Hierfür sind professionsspezifi- } \\
\text { sche Habitualisierungen und Handlungsdispositionen unerlässlich, da erst durch } \\
\text { eine Konkretisierung dieser professionseigenen Merkmale die zu klärenden Di- } \\
\text { vergenzen sichtbar werden. Diese Klärung sollte sich in einem stetig wiederho- } \\
\text { lenden Prozess befinden, da sich Rollenzuschreibungen in unregelmässigen Pe- } \\
\text { rioden verändern können. Deshalb ist es von Vorteil, wenn Stufenteams wö- } \\
\text { chentliche Treffen implementieren oder Q-Gruppen den Fokus stärker auf solche } \\
\text { Thematiken legen, um Prozesse zur Auflösung professionsbezogener Span- } \\
\text { nungsfelder voranzutreiben. Der Austausch der Stufenteams sollte idealerweise } \\
\text { im Konzept der Tagesschule konkretisiert und mit Massnahmen versehen sein, } \\
\text { welche doppelte Zielkongruenzen, unterschiedliche Handlungslogiken und un- } \\
\text { präzise Abgrenzungen sowie Rollenunschärfe verhindern. }\end{array}$ \\
\hline \multirow[b]{2}{*}{$\begin{array}{l}\text { Kooperations- } \\
\text { probleme }\end{array}$} & $\begin{array}{l}\text { Darlegung des Problems } \\
\text { „Nicht hinreichend besprochene Aufgabenverteilung sowie Koordinationsschwä- } \\
\text { chen an Schnittstellen zwischen Betreuung und Schule gehen mit zu geringer } \\
\text { Kooperationszeit einher“ (Thimm, 2015, S. 35). }\end{array}$ \\
\hline & $\begin{array}{l}\text { Konzeptorientierte Reflexion } \\
\text { Die im Konzept beschriebenen Kooperationsgremien sind verbindlich und müs- } \\
\text { sen bis auf die informellen Gespräche regelmässig wahrgenommen werden. } \\
\text { Dadurch wird eine konzeptionelle Dringlichkeit propagiert, welche den Mitarbei- } \\
\text { tenden einerseits den Status von Kooperation verdeutlicht und anderseits genü- } \\
\text { gend Zeit zur Verfügung stellt, den darin enthaltenen Anforderungen nachzu- } \\
\text { kommen. }\end{array}$ \\
\hline
\end{tabular}


Dieses konzeptionelle Bekenntnis für mehr zeitliche Ressourcen kann nicht darüber hinwegtäuschen, dass der Faktor Zeit in der Praxiswahrnehmung beider Professionen eine unterschiedlich gedeutete Variable ist. In der Arbeitspraxis der Tagesschule hat sich gezeigt, dass eine konsequente Umsetzung des Konzepts über einen längeren Zeitraum einen Mehrwert sichtbar macht, der durch konstruktiven Austausch in Gremien weiter intensiviert werden kann.

\section{Beitrag zur Qualitätssteigerung durch Fachpersonen der Sozialen Arbeit durch interprofessionelle Kooperation}

Laut Horstkemper (2011, S. 134) zeigen empirische Befunde, dass eine hohe Intensität der Kooperation offensichtlich nicht automatisch zu positiven Bewertungen ebendieser führt. Als wichtiges Merkmal einer gelingenden Kooperation wird die Selbstwirksamkeit der Fachpersonen der Sozialen Arbeit aufgeführt, welche durch Partizipation zum Tragen kommt. Die Möglichkeit, aktiv in die Gestaltung und Konzeption von Tagesschulen eingebunden zu werden, beeinflusst die Intensität und führt zu einer positiven Bewertung der auf dem Partizipationsgedanken basierenden Kooperation.

Dies untermauert die Praxis der exemplarisch herangezogenen Tagesschule, welche den Fachpersonen der Sozialen Arbeit seit geraumer Zeit auf organisatorischer sowie struktureller Ebene Mitbestimmung, Selbstbestimmung und Selbstverwaltung zuspricht und dadurch ein hohes Mass an Selbstwirksamkeit generiert. Diese positiv bewertete Eigenwahrnehmung der Profession fördert zusätzlich die Zusammenarbeit innerhalb der Stufenteams. Dies ist wichtig, da die Stufenteams gemeinsame Wertesysteme für die zu führenden Doppelklassen aushandeln und erarbeiten müssen, damit das Potential beider Professionen in der Wechselwirkung maximal ausgeschöpft werden kann. Dabei bietet sich der Fokus auf Themen des sozialen Miteinanders und der Herstellung von sozialer Gerechtigkeit in Bezug auf Klassenklima, Konflikte, Werte, Beziehungen und Schulkultur an, da dort unter anderem die gemeinsame Handlungsbasis der beiden Professionen festzustellen ist und diese Themen durch den Habitus der Sozialen Arbeit geprägt und entsprechend weiterentwickelt werden können. Innerhalb dieser Prozesse können Fachpersonen der Sozialen Arbeit soziale Prozesse begleiten oder diese initiieren und dabei Formen des sozialen Lernens implementieren. Weiterführend kann durch einen intensiven Austausch zwischen Lehrpersonen und Fachpersonen der Sozialen Arbeit auch eine präventive Wirkung auf entstehende Konflikte erwirkt werden, da Unterricht und Betreuung nicht entkoppelt, sondern miteinander verwoben sind und dadurch eine ganzheitliche, multiperspektivische Sichtweise entsteht. Dabei sollte von Seiten der Fachpersonen der Sozialen Arbeit bewusst unter Verknüpfung formaler, non-formaler und informeller Bildungsangebote individuelle Förderung, Ent- 
wicklung von Kompetenzen und eine sinnvolle Freizeitgestaltung vorangetrieben werden.

Die positiven Aspekte von Kooperation können nicht darüber hinwegtäuschen, dass sich ebenso Spannungsfelder auftun, welche in direktem Bezug zum Berufsauftrag der jeweiligen Profession stehen. Die Fachpersonen der Sozialen Arbeit orientieren sich an Grundsätzen des Berufskodexes von AvenirSocial $^{13}$, wohingegen der Lehrkörper unter anderem auf Selektion und Sanktion zurückgreift und sich somit in ein Spannungsfeld begibt, das von den divergierenden Positionen der innerhalb von Kooperationsbeziehungen ausgehandelten Habitualisierungen und Handlungsdispositionen sowie den eigenen Verpflichtungen zur Bewertung, Benotung, Selektion und Sanktion geprägt ist (vgl. Beitrag von Thieme in diesem Sammelband). Als Teil des Schulsystems muss sich die Soziale Arbeit ebenfalls an den Prämissen dieses Systems orientieren und kann sich somit den Selektions- und insbesondere den Sanktionsfunktionen nur bedingt widersetzen und entziehen. Diese Tatsache eröffnet jedoch Chancen für einen professionsübergreifenden konstruktiven Dialog, wie mit diesen Paradoxien umgegangen werden kann.

Die aufgeführten Elemente können durch eine konsequente Umsetzung und Einforderung seitens der Fachpersonen der Sozialen Arbeit in Kombination mit bildungspolitischen Bestrebungen zu einem merklichen Qualitätsgewinn innerhalb einer auf Kooperation ausgelegten Tagesschule führen.

\section{Fazit}

In diesem Beitrag wurde ein Konzept einer auf Kooperation ausgerichteten Tagesschule aufgegriffen, welche Partizipation der verschiedenen Professionen konzeptuell verankert und mit empirischen Erkenntnissen sowie reflexiven Erfahrungswerten basierend auf dem spezifischen Praxisalltag einer exemplarischen Tagesschule vermengt. Die sich daraus entwickelnden Fragen sowie Dilemmata verstehen sich als logische Konsequenz einer intensiven Kooperation, aufgrund welcher im Umkehrschluss wieder durch Kooperation tragfähige Lösungen für alle betroffenen Parteien kreiert werden können. Die im Beitrag erwähnte Selbstwirksamkeit als zentraler Aspekt einer partizipativen Kooperation hat für die Kooperation und darauf aufbauend für die Qualität einer Tagesschule eine essenzielle Bedeutung. Deshalb sollten Fachpersonen der Sozialen Arbeit partizipative Bedingungen in Verzahnung mit bildungspolitischen Forderungen, welche diese Selbstwirksamkeit ermöglichen, prononciert verlangen.

13 Vgl. AvenirSocial (2010). Berufskodex Soziale Arbeit Schweiz. Ein Argumentarium für die Praxis der Professionellen. Bern: AvenirSocial. 
Das daraus erwachsende Selbstverständnis sollte von einer grundlegenden konstruktiven, ziel-, lösungs- und ressourcenorientierten Haltung, welche zusätzlich durch soziale Werte und positive Einstellungen gegenüber Kooperation geprägt ist, von allen Beteiligten des Systems Tagesschule gelebt werden, um das vorhandene Potential der Profession Sozialer Arbeit maximal nutzen zu können. Dafür sind jedoch Fachpersonen der Sozialen Arbeit vonnöten, welche dieses Potential erkennen und propagieren.

\section{Literatur}

Hart, R. (1997). Children's Participation. The Theory and Practice of Involving Young Citizens in Community Development and Environmental Care. New York: United Nations Children's Fund.

Horstkemper, M. (2011). Multiprofessionelle Kooperation an Ganztagesschulen. Herausforderung und Chance für Schul- und Professionsentwicklung. In K. Speck, T. Olk, O. Böhm-Kasper, H. J. Stolz \& C. Wiezorek (Hrsg.), Ganztagsschulische Kooperation und Professionsentwicklung. Studien zu multiprofessionellen Teams und sozialräumlicher Vernetzung (S. 128-137). Weinheim: Juventa.

Kuster, R. \& Bussmann, E. (2017). Kooperation (Arbeitsbuch 3.) In Brückel F.; Kuster, R.; Annen, L.; Larcher, S. (Hrsg.), Qualität in Tagesschulen/ Tagesstrukturen (QuinTaS). Bern: hep-Verlag.

Schüpbach, M. (2010). Ganztägige Bildung und Betreuung im Primarschulalter. Qualität und Wirksamkeit verschiedener Schulformen im Vergleich. Wiesbaden: VS.

Speck, K., Olk, T., Böhm-Kasper, O., Stolz, H. J. \& Wiezorek, C. (2011). Multiprofessionelle Teams und sozialräumliche Vernetzung? Befunde zur Ganztagsentwicklung. In K. Speck, T. Olk, O. Böhm-Kasper, H. J. Stolz \& C. Wiezorek (Hrsg.), Ganztagsschulische Kooperation und Professionsentwicklung. Studien zu multiprofessionellen Teams und Sozialräumlicher Vernetzung (S. 7-28). Weinheim: Juventa.

Speck, K., Olk, T. \& Stimpel, T. (2011). Professionelle Kooperation unterschiedlicher Berufskulturen an Ganztagesschulen. Zwischen Anspruch und Wirklichkeit. In K. Speck, T. Olk, O. Böhm-Kasper, H. J. Stolz \& C. Wiezorek (Hrsg.), Ganztagsschulische Kooperation und Professionsentwicklung. Studien zu multiprofessionellen Teams und sozialräumlicher Vernetzung (S. 69-84). Weinheim: Juventa.

Thimm, K. (2015). Soziale Arbeit im Kontext Schule. Reflexion, Forschung und Praxisimpulse. Weinheim: Beltz Juventa.

Tillmann, K. \& Rollett, W. (2011). Multiprofessionelle Kooperation und Partizipation an Ganztagesschulen. Welche Auswirkungen hat die strukturelle Einbindung des weiteren pädagogisch tätigen Personals auf die berufsgruppenüberreifende $\mathrm{Zu}$ sammenarbeit? In K. Speck, T. Olk, O. Böhm-Kasper, H. J. Stolz \& C. Wiezorek (Hrsg.), Ganztagsschulische Kooperation und Professionsentwicklung. Studien zu multiprofessionellen Teams und sozialräumlicher Vernetzung (S. 29-47). Weinheim: Juventa. 


\title{
Schulsozialarbeit und Schulentwicklung - eine Betrachtung aus der Perspektive der Schulsozialarbeit
}

\author{
Aldo Venzi
}

Inwiefern kann oder soll die Schulsozialarbeit (SSA) Einfluss auf die Entwicklungsprozesse einer Schule nehmen? Diese Fragestellung wird im vorliegenden Artikel aus empirischer Sicht sowie anhand eines Praxisbeispiels aus der Perspektive der SSA betrachtet und diskutiert. Ein Blick auf die aktuelle Literatur zeigt, dass nur wenige Arbeiten zu dieser spezifischen Fragestellung vorliegen und darin unterschiedliche Standpunkte vertreten werden.

Ist Schulentwicklung eine primäre Aufgabe der SSA oder ist sie als untergeordnete Aufgabe, bestenfalls als ein Nebenprodukt oder eine Begleiterscheinung, einzuordnen? Diese Frage wird in der Literatur kontrovers diskutiert (Holtbrink \& Kastirke, 2013, S. 100ff.). Es gilt diesbezüglich zu erwähnen, dass im deutschsprachigem Raum unterschiedliche Definitionen von Schulentwicklung existieren; zudem werden verschiedene Begriffe synonym verwendet, die jedoch allesamt eher vage bleiben (ebd., S. 102f.).

Gemäss Holtbrink und Kastirke nimmt die SSA in der Schulentwicklung in der deutschen Bildungslandschaft eine bedeutende Rolle ein, insbesondere bezüglich aktueller Themen, wie z.B. der Inklusion oder Ganztagsbildung. Die Autorinnen verstehen die Funktion der SSA als Schnittstelle zwischen Jugendhilfe und Schule und sprechen in diesem Zusammenhang von einer multiprofessionellen Schule (Holtbrink \& Kastirke, 2013, S. 100ff.; Holtbrink, 2015, S. 36ff.).

Ein Blick auf die Praxis in der Schweiz zeigt, dass die SSA gerade bei diesen aktuellen Themen mancherorts ausgeschlossen bleibt. Auch in den konzeptionellen Verankerungen sind unterschiedliche Standpunkte erkennbar: So wurde die Schulentwicklung z.B. im ersten Konzept der SSA der Stadt Zürich, das im Jahre 2003 definitiv implementiert wurde, als Aufgabe der SSA ausgeschlossen. An dieser Auslegung wird bis heute festgehalten. Im Leitbild für die Soziale Arbeit in der Schule des Berufsverbandes für Soziale Arbeit der Schweiz (AvenirSocial, 2016) wird hingegen sowohl bei den 
Zielen als auch bei den Aufgaben darauf hingewiesen, dass die SSA aktiv auf die Schulentwicklung einwirken und die Schule darin unterstützen soll.

\section{Hat die Schulsozialarbeit aus empirischer Sicht einen Einfluss auf die Schulentwicklung?}

Holtbrink und Kastirke erläutern ein Schulentwicklungsmodell von Rolff (2010), das auch einen hilfreichen Orientierungsrahmen für die SSA bietet. Das Modell beinhaltet drei Elemente bzw. Subsysteme: die personale Entwicklung, die Unterrichtsentwicklung und die Organisationsentwicklung. Diese Subsysteme bedingen sich gegenseitig und stehen gleichzeitig in einer Wechselwirkung mit ihrem Umfeld. Systemisch betrachtet ergeben Veränderungen in einem Subsystem unvermeidlich Veränderungen in den anderen Subsystemen. So wirken sich personale Entwicklungen, wie z.B. in Form von Beratungen der Lehrpersonen (LP), ebenfalls auf die Unterrichts- und Organisationsentwicklung aus (Rolff, 2010; zit. nach Holtbrink \& Kastirke, 2013, S. 103ff.).

Im Rahmen einer empirischen Untersuchung wurde der Einfluss der SSA auf die Schulentwicklung in diesen drei Subsystemen erhoben (Holtbrink \& Kastirke, 2013). Gemäss dieser Studie wirkt sich die SSA auf alle drei Subsysteme aus, wenn auch mit unterschiedlichen Ausprägungen. Der stärkste Einfluss der SSA ist im Bereich der Unterrichtsentwicklung erkennbar. Dabei stehen nicht didaktische oder methodische Kompetenzen der LP (Verbesserung des Unterrichtes), sondern Verhaltensveränderungen der Schülerinnen und Schüler (SuS) im Vordergrund. Die SSA wirkt durch ihre Beratungstätigkeit auf die SuS und durch kollegiale Beratung auf die LP ein. Dadurch können z.B. Unterrichtsstörungen nachweislich verringert werden. Auch durch die Entwicklung spezifischer Unterrichtseinheiten zu Themen wie z.B. Sexualität, Sucht oder Gewalt übt die SSA Einfluss auf die Unterrichtsentwicklung aus (S. 105ff.).

Im Subsystem der personalen Entwicklung werden die Personalfortbildung, -führung und -förderung zusammengefasst. Gemäss der Studie ist der Einfluss der SSA in diesem Subsystem geringer und findet am ehesten in der Personalfortbildung statt. Dort kann die SSA Impulse für die Erarbeitung von spezifischen Unterrichtskonzepten einbringen. Auch im Subsystem Organisationsentwicklung können konkrete Einflussbereiche definiert werden: Die SSA kann z.B. auf institutioneller Ebene auf die konzeptionelle Schulentwicklung (Steuergruppen, Konferenzen usw.) einwirken (Holtbrink \& Kastirke, S. 109ff.). Einen weiteren Bereich der Einflussnahme sehen die Autorinnen in der Vernetzung mit verschiedenen Institutionen ausserhalb des Sys- 
tems Schule. Die SSA stellt dabei eine Verbindung zwischen den verschiedenen Angeboten der Sozialen Arbeit, der Schule und dem Arbeitsmarkt dar und gewährleistet durch ihr spezifisches Wissen sowie ihre sozialraumorientierte Vernetzung eine optimale Unterstützung der SuS. Als letzter Bereich, auf den die SSA Einfluss nimmt, werden die Schulkultur und das Schulklima erwähnt (S. $111 \mathrm{ff}$.).

In den Ausführungen von Holtbrink und Kastirke (2013) wird deutlich, dass die SSA Einfluss auf Schulentwicklungsprozesse nehmen kann, wobei dieser massgeblich von der fachlichen und organisationalen Verankerung in der jeweiligen Schuleinheit abhängig ist. Der Einfluss der SSA wird entscheidend vom Kooperationsverhältnis zu den LP sowie der Beziehung zur Schulleitung (SL) und deren Unterstützung beeinflusst. Zugleich ist die Eigeninitiative der SSA wesentlich: Je aktiver die SSA ihren Gestaltungsraum nutzt, umso mehr Einflussmöglichkeiten hat sie (Holtbrink \& Kastirke, 2013, S. 113f.; Holtbrink, 2015, S. 36ff.).

\section{Ein Praxisbeispiel: Die Implementierung von Konfliktlotsen}

Die Einflussnahme der SSA auf Schulentwicklungsprozesse lässt sich z.B. bei der Implementierung von Konfliktlotsen aufzeigen. Exemplarisch wird im Folgenden der systemische Entwicklungsprozess des Konfliktmanagements an der Schule Riedtli ${ }^{1}$ in Zürich aufgezeigt. ${ }^{2}$

Das Praxisbeispiel orientiert sich am eingangs erwähnten Konzept der SSA der Stadt Zürich, das von den Sozialen Diensten in Kooperation mit dem Schul- und Sportdepartement der Stadt Zürich erarbeitet wurde. Fachlich und strategisch ist die SSA darin der Jugend- und Familienhilfe der Sozialen Dienste unterstellt und nicht wie mancherorts den Schulen, der Schulbehörde usw. In Bezug auf die Schulentwicklung gilt in diesem Praxisbeispiel jedoch die Schuleinheit ${ }^{3}$, in der sowohl die SL als auch die SSA direkt und aktiv Einfluss nehmen können, als Orientierungssystem.

In den vergangenen Jahren implementierten verschiedene Schulen der Stadt Zürich mit Unterstützung der Fachstelle für Gewaltprävention (FGP) ${ }^{4}$ das Konfliktlotsen-Modell. Die Schule Riedtli entschied sich aufgrund aktueller Gewaltvorfälle für eine Teilnahme. Das Modell orientiert sich an den

\footnotetext{
Der Name der Schule darf mit Einverständnis der Schulleitung verwendet werden.

Der Autor war mehrere Jahre als SSA in dieser Schuleinheit tätig.

Eine Schuleinheit kann mehrere Kindergärten, Schulen und Horte umfassen.

Die Fachstelle für Gewaltprävention ist eine Abteilung des Schul- und Sportdepartementes der Stadt Zürich.
} 
schüler- und handlungsorientierten Unterrichtsmethoden von Hagedom (2005), welche an diversen Schulen in Berlin erfolgreich eingesetzt werden. Das Konfliktlotsen-Modell ist als ein Peer-to-Peer-Mediationsmodell anzusehen, in dem die sozialen Kompetenzen von ausgewählten Schülerinnen und Schülern (SuS) im Rahmen der Ausbildung gestärkt werden, um sie zu befähigen, bei Konflikten zwischen Schulkindern deeskalierend und vermittelnd zu intervenieren. Dafür werden Methoden mit ausgeprägten partizipativen Ansätzen verwendet. Durch die Peer-Mediation werden auch die sozialen Kompetenzen der anderen SuS gestärkt, was die gesamte Schulhauskultur und das Schulklima positiv beeinflusst.

Die Schule Riedtli orientierte sich im Rahmen der Implementierung des Modells im Jahr 2009 ergänzend zu den Ausführungen von Hagedom (2005) an den systemischen Grundannahmen von Faller (1998) und Rademacher (2009). Beide Autoren vertreten die Meinung, dass Peer-Mediation nur eine Chance auf eine nachhaltige Wirkung hat, wenn sie Teil eines gesamten systemischen Veränderungsprozesses ist und in das Konfliktmanagement der Schule integriert wird.

Um diesen systemischen Veränderungsprozess zu gestalten und die Struktur des bestehenden Konfliktmanagements zu analysieren, kann der Ansatz des pädagogischen Hexagons nach Faller (1998) beigezogen werden. Es beinhaltet sechs Elemente: konstruktive Konfliktbearbeitung, begleitende Programme, soziale Lernziele im Unterricht, Trainings zum Lehrpersonenverhalten, Öffnung der Schule sowie Prävention im Schulprogramm. Diese Elemente müssen unabhängig voneinander bearbeitet werden, stehen jedoch in einer Wechselwirkung zueinander (S. 76f.).

In einer ersten Entwicklungsphase fand in Kooperation mit der FGP die Konzeption und Durchführung der Konfliktlotsen-Ausbildung der SuS statt. Auf inhaltlicher/personeller/struktureller Ebene mussten dazu verschiedene Massnahmen an der Schule umgesetzt werden, damit die Konfliktlotsen zeitnah ihre Tätigkeit aufnehmen konnten. Dafür war ein Entwicklungsplan zur Implementierung des „Subsystems Konfliktlotsen“ auf organisationaler Ebene notwendig, um dieses inhaltlich und strukturell mit allen bestehenden Subsystemen (Klassen- und Schulhausparlament, Präventionsprojekte usw.) $\mathrm{zu}$ „verweben“. Dabei galt es darauf zu achten, dass sich das „Subsystem Konfliktlotsen" verankern, festigen und weiterentwickeln kann und nicht von der Schuleinheit ,abgestossen“ wird. Es gilt zu erwähnen, dass die Implementierung des Modells aufgrund der natürlichen Fluktuation der SuS in der Schule auch immer einen Wettlauf gegen die Zeit darstellte. Dieser komplexe und dynamische Prozess dauerte einige Jahre und erforderte fortlaufende Überprüfungen und Abstimmungen. 
Diese erste Phase zeigte eine nachhaltige Wirkung: Das Angebot der Peer-Mediation wurde von den SuS aktiv genutzt. Die Konfliktlotsen erhielten in den ersten Jahren zunehmend Mediationsaufträge innerhalb der Schuleinheit. In der Folge war ein Rückgang der Gewalt an der Schule zu vermerken, der von allen Beteiligten wahrgenommen wurde. Die positiven Auswirkungen während der ersten drei Jahre führten zur nächsten Entwicklungsphase, welche den Fokus auf die Peer-Education ${ }^{5}$ legte. Das vordringliche Ziel bestand darin, dass die Konfliktlotsen ihr erworbenes Wissen und ihre Erfahrungen an andere SuS weitergeben, sodass die Handlungsansätze der Partizipation bei allen SuS der Schule eine Wirkung entfalten.

Diese Phase war von zwei Entwicklungsschritten geprägt. Der erste richtete die Aufmerksamkeit auf Unterrichtseinheiten zur Förderung der sozialen Kompetenzen, die in Kooperation mit den Konfliktlotsen entwickelt wurden. Die inhaltliche und methodische/didaktische Konzeption wurden dabei massgeblich von diesen definiert. In der Folge unterrichteten die Konfliktlotsen die SuS zu Themen wie Gewaltfreie Kommunikation, Impulskontrolle oder positive Funktionen von Affekten.

In einem zweiten Schritt wurde der Fokus auf den Bereich der Krisenintervention gelegt. Es wurden wiederum in Zusammenarbeit mit den Konfliktlotsen Konzepte für Klasseninterventionen ausgearbeitet, um deren Kompetenzen auf der Primarstufe einzusetzen. Die Konfliktlotsen wurden in entsprechenden Unterrichtssequenzen auf ihre Funktion und Rollen im Rahmen von Klasseninterventionen vorbereitet und führten in der Folge in Primarklassen anderer Schulhäuser verschiedene Klasseninterventionen zu Themen wie Zivilcourage oder Respekt durch.

Die Umsetzung der skizzierten Phasen verlief selbstverständlich nicht ohne kritische Zwischenfälle, wie z.B. disziplinarisch-grenzüberschreitende Verhaltensweisen von Konfliktlotsen, die eine Auseinandersetzung und Klärung hinsichtlich Haltungsfragen der Schulteams oder klärende Interventionen erforderten. In diesem Zusammenhang mussten z.B. auch Fragen zu Sanktionen oder Ausschlüssen von Konfliktlotsen geklärt werden. Dabei musste stets umsichtig zwischen der individuellen Klärung der Situation und einer potentiellen Gefährdung der Reputation des Konfliktlotsen-Modells abgewogen werden. Die strategische Steuerung des gesamten Entwicklungsprozesses wurde von der SL in Kooperation mit der SSA, die operative Steuerung und fachliche Begleitung der Konfliktlotsen während des Schuljahres von der SSA durchgeführt und verantwortet.

Im Rahmen der Implementierungsphase wurden keine strukturierten Evaluationen nach wissenschaftlichen Kriterien umgesetzt, jedoch in den ersten

5 Die Schule verwendete synonym für Peer-Education den Begriff des Peer-Involvements. 
Jahren verschiedene Befragungen der Konfliktlotsen und SuS der Schuleinheit durchgeführt. Im Rahmen der jährlich stattfindenden Q-Tage ${ }^{6}$ wurde das Modell innerhalb des Schulteams wiederkehrend ausgewertet. Die Evaluationen dieser Befragungen führten „organisch“ zu weiteren Entwicklungsphasen des Modells, die mit einer sukzessiven Ausweitung des Einflusses des „Subsystems Konfliktlotsen“ innerhalb des ganzen Schulsystems einhergingen. Die Konfliktlotsen nahmen z.B. bei der Gestaltung von Elternabenden, Projekttagen und schulinternen Anlässen zunehmend eine bedeutende Rolle ein. Um die Qualität ihrer Begleitung sicherzustellen, wurde das KonfliktlotsenModell fortan von den LP und der SSA, die Peer-Education von den Fachpersonen der Betreuung begleitet ${ }^{7}$.

Peer-Education wird seit vielen Jahren erfolgreich in Schulen eingesetzt, insbesondere in der Suchtprävention sowie in der Gesundheits- und Sexualerziehung. Evaluationen von Peer-Projekten zeigen bei den Peer-Educators positive Ergebnisse hinsichtlich der Zunahme ihrer themenspezifischen Kompetenzen, der Stärkung ihrer Selbstwirksamkeit und in der Folge ihres Selbstwertes. Die anderen SuS werden durch die Peer-Education in ihrer sozialen und kognitiven Entwicklung gefördert (Faller, 1998, S. 58ff.; Polleichtner, 2015, S. 35ff.). Die Selbstevaluation der Schule Riedtli weist darauf hin, dass bei den Konfliktlotsen durch die Peer-Education eine Zunahme spezifischer Kompetenzen sowie eine Stärkung der Selbstwirksamkeit erzielt und bei den SuS die soziale Entwicklung gefördert wurden.

In der Literatur werden solche Partizipationsprogramme ${ }^{8}$ kritisch diskutiert. Damit die Integration von sozial benachteiligten Kindern und Jugendlichen durch Teilhabe ermöglicht wird, sollte auch deren soziale Benachteiligung berücksichtigt werden, fordern die Kritikerinnen und Kritiker. In diesem Zusammenhang werden solche Programme z.T. als zu ,sprachlastig“ und abstrakt eingeschätzt. Dies hat zur Folge, dass sie oft auf Kinder und Jugendliche aus bildungsnahen, sozial privilegierten Familien ausgerichtet sind und dadurch nicht zu einer effektiven Teilhabe, sondern zu einer Exklusion sozial benachteiligter Kinder und Jugendlichen führen (Biebricher, 2011, S. 225ff.). Um eine Durchmischung der individuellen soziokulturellen Voraussetzungen der SuS sicherzustellen, hat die Schule Riedtli als Konsequenz ein entsprechendes Auswahl- und Aufnahmeverfahren entwickelt.

Vor dem Hintergrund des pädagogischen Hexagons nach Faller fand das Training des Lehrpersonenverhaltens im Praxisbeispiel nur eine untergeord-

6 Der Q-Tag beinhaltet Teamfortbildungen und Organisationsentwicklung. Jede Schuleinheit ist der Kreisschulpflege in den Jahresberichten diesbezüglich rechenschaftspflichtig.

7 Fachpersonen der Betreuung können Fachpersonen der Sozialen Arbeit, sozialpädagogische Fachkräfte, Fachpersonen Betreuung, Betreuungsassistenzen sein (vgl. Einleitung).

8 Peer-Education kann als Handlungsansatz der Partizipation angesehen werden. 
nete Beachtung. Doch obwohl es kaum bewusst bearbeitet wurde, übte die Stärkung der SuS bezüglich des Themas einen „bottom-up“-Einfluss auf das gesamte System und damit auch auf das Verhalten der LP aus. Ein Beispiel dafür war ein Vorfall im Rahmen einer Durchführung der KonfliktlotsenAusbildung, wo es Widerstände von Seiten der SuS gab. Zentraler Konfliktpunkt war das Verhalten gewisser LP gegenüber den SuS, das anscheinend durch Abwertungen, Beleidigungen u.a. gekennzeichnet war. Die Konfliktlotsen waren dezidiert der Ansicht, dass einige LP Schwächen bezüglich ihrer eigenen Konfliktfähigkeit zeigen. In der Folge formulierten sie eine Charta mit ihren Erwartungen und überreichten diese der SL. Diese nahm diese Charta ernst und führte eine SuS-Gesamtbefragung hinsichtlich dieser Vorwürfe durch. Die Vorwürfe wurden von den anderen SuS bestätigt, was das System stark „erschütterte“. Einige LP vertraten dabei die Ansicht, dass den Konfliktlotsen dadurch zu viel Macht und Einfluss gewährt wurde. Die Ergebnisse der SuS-Befragung wurden jedoch noch von einer zeitnah folgenden externen Schulevaluation ${ }^{9}$ erhärtet.

Abschliessend kann festgehalten werden, dass die sechs Elemente des pädagogischen Hexagons in einer untrennbaren Wechselwirkung zueinanderstehen und sich ein System auch dann verändert, wenn nicht alle Elemente gleichermassen bearbeitet werden.

\section{Abschliessende Überlegungen zur Rolle der SSA in der Schulentwicklung}

Die empirischen Erkenntnisse und die Ergebnisse des systemischen Entwicklungsprozesses im selbstevaluierten Fallbeispiel stehen grundsätzlich nicht im Widerspruch zueinander. Es kann festgehalten werden, dass die SSA im Fallbeispiel in Anlehnung an das Schulentwicklungsmodell von Rolff (2010) im Subsystem der Organisationsentwicklung einen Einfluss auf die Schulkultur und das Schulklima nehmen konnte. Es ist zudem erkennbar, dass im Entwicklungsprozess auch Aspekte des Subsystems Unterrichtsentwicklung (spezifische Unterrichtseinheiten) und personale Entwicklung (spezifische Unterrichtskonzepte) miteinflossen, was durch die Wechselwirkung der drei Subsysteme $\mathrm{zu}$ erklären ist. Wünschenswert sind weitere Untersuchungen zum Einfluss der SSA auf die Schulentwicklung, um aufgrund der Erkenntnisse u.a. die Aufgaben und Ziele der SSA im Kontext der jeweiligen Schule zu konkretisieren.

9 Weiterführende Informationen: www.vsa.zh.ch 
Im Kontext des föderalistischen Bildungssystems der Schweiz zeigt sich in Bezug auf die fachliche und organisationale Verankerung der SSA ein vielfältiges Bild. Der Einfluss der SSA auf die Schulentwicklung ist massgeblich von den Bedingungen der jeweiligen Schuleinheit abhängig. Es ist jedoch wesentlich, dass unabhängig von dieser Tatsache ein Weg gefunden wird, um die gesellschaftlichen Herausforderungen und Veränderungen der Schulen zwischen beiden Professionen partnerschaftlich zu diskutieren und zu gestalten.

Insbesondere auf handlungspraktischer Ebene bestehen diesbezüglich konkrete Möglichkeiten. In der Stadt Zürich werden z.B. von den Leitungspersonen der Jugend- und Familienhilfe der Sozialen Dienste sowie den SL Jahresziele für die SSA festgelegt. Bei der Diskussion dieser Jahresziele sollten Schulentwicklungsprozesse vermehrt berücksichtigt und ein langfristiger Planungshorizont von mehreren Jahren fokussiert werden, wobei das Schulentwicklungsmodell von Rolff einen sinnvollen Orientierungsrahmen bieten kann. Das verwendete Praxisbeispiel war z.B. über mehrere Jahre Teil der Jahresziele der SSA und wurde kontinuierlich weiter konkretisiert.

Dem Kooperationsverhältnis zwischen SL, LP und SSA als zentraler Aspekt muss ein wichtiger Stellenwert zugesprochen werden. Ein Blick in die aktuelle Praxis zeigt, dass das Kooperationsverhältnis bezüglich des Einflusses der SSA auf Schulentwicklungsprozesse ein wesentliches Merkmal für den Erfolg darstellt. Der positive Entwicklungsprozess im verwendeten Praxisbeispiel wurde entscheidend durch das partnerschaftliche Kooperationsverhältnis zwischen den LP, der SL und der SSA beeinflusst. Es wurde zudem deutlich, dass die Einflussnahme der SSA auf Schulentwicklungsprozesse auch von der Eigeninitiative der SSA, also dem Selbstverständnis jeder/jedes Schulsozialarbeitenden abhängig ist. Es ist davon auszugehen, dass Kooperationsverhältnis und Eigeninitiative korrelieren und sich gegenseitig beeinflussen. Es lässt sich daher der Anspruch an die SSA formulieren, sich bewusst und aktiv an Schulentwicklungsprozessen zu beteiligen und dadurch einen Beitrag an eine multiprofessionelle Schule zu leisten. Aus Sicht der Sozialen Arbeit - verstanden als Profession, die sich für soziale Gerechtigkeit einsetzt und sich an den Prinzipien der Menschenrechte orientiert - steht die SSA daher gewissermassen in der Pflicht, zum Wohle der SuS Einfluss auf die Schulentwicklung zu nehmen, wie im verwendeten Praxisbeispiel über Handlungsansätze der Partizipation. Die SSA verfügt jedoch über ein viel breiteres fachliches und methodisches Potential, das es zur Entwicklung einer Schuleinheit einzubringen gilt - die SSA ist mehr als eine Feuerwehrübung und ein Einzelfallhilfe-Pflaster. 


\section{Literatur}

AvenirSocial (2016). Leitbild Soziale Arbeit in der Schule. Bern, Luzern: AvenirSo$\mathrm{cial} / \mathrm{SSAV}$.

Biebricher, M. (2011). Partizipation von Schülerinnen und Schülern - Herausforderungen und Handlungsansätze für die Schulsozialarbeit. In F. Baier \& U. Deinet (Hrsg.), Praxisbuch Schulsozialarbeit. Methoden, Haltungen und Handlungsorientierungen für eine professionelle Praxis. Opladen: Barbara Budrich.

Drilling, M. (2007). Schulsozialarbeit. Antworten auf veränderte Lebenswelten (3. Aufl.). Bern: Haupt Verlag.

Faller, K. (1998). Mediation in der pädagogischen Arbeit. Ein Handbuch für Kindergarten, Schule und Jugendarbeit. Müllheim: Verlag an der Ruhr.

Hagedom, O. (2005). Mediation - durch Konflikte lotsen. 58 schüler- und handlungsorientierte Unterrichtsmethoden. Stuttgart, Leipzig: Ernst Klett Schulbuchverlag.

Holtbrink, L. (2015). Neue Herausforderungen für die Schulsozialarbeit im Rahmen des Inklusionsprozesses. In Sozialmagazin 11.-12.2015, S. 31-38.

Holtbrink, L. \& Kastirke, N. (2013). Schulsozialarbeit im Kontext Schulentwicklung. In A. Spies (Hrsg.), Schulsozialarbeit in der Bildungslandschaft. Beiträge zur Sozialen Arbeit an Schulen. Wiesbaden: Springer Fachmedien.

Polleichtner, I. (2015). Peer-Education in der schulischen Suchtprävention. SuchtMagazin 2/2015, S. 35-38.

Rademacher, H. (2009). Mediation in der Schule. Unveröff. Skript. CAS Mediation. Zürcher Hochschule für angewandte Wissenschaften, Departement Soziale Arbeit.

Rolff, H. G. (2010). Schulentwicklung als Trias von Organisations-, Unterrichts- und Personalentwicklung. In T. Bohl (Hrsg.), Handbuch Schulentwicklung. TheorieForschung-Praxis (S. 29-36). Stuttgart: UTB. 


\title{
„Chance und Herausforderung mit Fachpersonen Tagesstrukturen in der Schule zu kooperieren“. Soziale Arbeit in der Schule aus der Sicht einer Schulleiterin
}

\author{
Karin Vaneck
}

Das Thiersteiner Schulhaus liegt im Basler Gundeldingerquartier („Gundeli“). Dieser Teil Basels steht mit nahezu 19.000 Einwohnern, nur 1,2 $\mathrm{km}^{2}$ und einem Fremdsprachenanteil von über 50\% hohen sozialen Herausforderungen gegenüber. Die Schule gilt als „Brennpunktschule“ und hat enorme Anforderungen zu meistern, wie beispielsweise ungenügende Deutschkenntnisse, soziale Probleme oder schwierige familiäre Verhältnisse auszugleichen. Es ist offensichtlich, dass eine Schule mit den alten Schulstrukturen - z.B. eine rigide Stundentafel oder Lehrmittelzwang - den Lernbedürfnissen der Heranwachsenden und den Lebenslagen ihrer Familien nicht gerecht werden kann.

Die Schulsozialarbeit (SSA), die in der Schule fest verankert ist, übernimmt hier eine tragende Rolle. Kinder, Eltern und Lehr- und Fachpersonen Tagesstrukturen können sich an die SSA wenden. Sie ist die Kontaktperson zu den schulnahen Diensten wie Kinder- und Jugenddienst, Kinder- und Erwachsenenschutzbehörde (KESB) oder Familienberatung/-begleitung. Sie arbeitet konkret mit allen Beteiligten, tauscht sich mit Fachpersonen unterschiedlicher Themenfelder aus und gibt Empfehlungen ab. Sie entlastet nicht nur Lehr- und Fachpersonen Tagesstrukturen, sondern auch die Schulleitung und die angehängten Dienste (Schulpsychologe, Tagesstruktur, KITA etc.).

Doch welche Herausforderungen sind es, die uns täglich vor grosse Probleme stellen?

Zunächst ist der hohe Fremdsprachenanteil zu erwähnen, der in der Schule noch viel spürbarer ist als im restlichen Quartier. Viele Migranten- und Flüchtlingsfamilien sind in den letzten Jahren ins Gundeli gezogen (Statistisches Amt Basel-Stadt, 2017a). Ein grosser Teil dieser Familien weist einen tiefen bis sehr tiefen Bildungsstand aus (Statistisches Amt Basel-Stadt, 2017b). Neben wenig bis keinen Deutschkenntnissen kämpfen die Familien mit Geldsorgen und behördlichen Ansprüchen, denen sie oft nicht genügen können. Die Leidtragenden sind dabei die Kinder dieser Familien, die oft 
weder ihre Muttersprache noch Deutsch lernen. Die schlechten Sprachkenntnisse der Eltern betreffen die Kinder ganz direkt. Der Anteil der logopädischen Befunde ist an der Primarschule Thierstein in den vergangenen 5 Jahren von 23 auf 48 Kinder pro Jahr gestiegen. Deren Schullaufbahn ist somit meist schon vorgezeichnet. Kinder mit einem logopädischen Befund erreichen bis Ende des 8. Schuljahres die Lernziele in den Sprachen nur ungenügend. Die Lehr- und Fachpersonen Tagesstrukturen der Mittelstufe versuchen bei vielen Kindern die Defizite durch einen Nachteilsausgleich oder gar individuelle Lernziele, welche zeugnisrelevant sind, abzufedern.

Wenig Deutschkenntnisse zu Beginn der Schulzeit, grosse Wissensdefizite nach den ersten Schuljahren, soziale Probleme wie bspw. wenig ausserfamiliäre Kontakte oder überforderte alleinerziehende Mütter präsentieren sich verstärkt, weil jene Kinder wenig oder keine Unterstützung durch die Eltern erhalten. Die Folge ist, dass die betroffenen Kinder keinen genügenden Schulabschluss erreichen und keine passende Ausbildung möglich wird. Dies kann längerfristig für jene Heranwachsenden heissen, dass eine Unterstützung der Sozialhilfe die logische Folge ist (EDI, BSV, Oktober 2016, Leitfaden: Kriterien wirksamer Praxis in der frühen Förderung).

Um diesen Teufelskreis zu durchbrechen, machte ich mich mit Lehrpersonen und dem Leiter Tagesstrukturen vor fünf Jahren auf den Weg, eine Bildungslandschaft zu werden.

\section{Bildungslandschaften}

Im Jahr 2012 konnten wir uns auf eine Ausschreibung für das Projekt „Bildungslandschaften Schweiz" bewerben, das die Jacobs Stiftung (2015) und der Kanton Basel Stadt lancierten.

Dieses Projekt wollten wir gemeinsam mit Tagesstruktur und Unterricht angehen. Der Leiter der Tagesstrukturen, ein ausgebildeter Sozialpädagoge, war deshalb als Co-Projektleiter neben der Schulleiterin von Beginn an dabei.

Die Hauptziele des Projektes waren, zusammen mit den Tagesstrukturen die Integration und Partizipation der Kinder und deren Familien im Kontext Schule zu verbessern und die Bildungschance jedes einzelnen Kindes zu optimieren. Dabei ging es zum einen darum, die Sprachkompetenzen von Eltern und Kindern sowie die Erziehungskompetenzen der Eltern zu stärken. Zum anderen wollten wir eine gemeinsame pädagogische Haltung aller Beteiligten im Schulhaus und eine positive Schulhauskultur aufbauen.

Im Quartier gab es bereits eine bunte Mischung vieler Angebote für Familien und Kinder. Nennenswert hierbei sind die Angebote der Robi-SpielAktionen oder der Stadtbibliotheken GGG, die neben vielen anderen in der 
Quartierkoordination („QuKo Gundeli“) vernetzt waren. Die QuKo ist ein Zusammenschluss von über 80 Vereinen im Quartier, der bestehende Angebote mit Neuem verknüpft. Bisher war die Schule dort jedoch nicht involviert, da die Schule als öffentlich rechtliche Einrichtung nicht als Verein auftreten kann. Bereits bestehende Angebote wie Sprachkurse für Eltern, Femmes-Tische oder Spielgruppenangebote sollten also in der Schule etablierter und bekannter werden.

Zusätzlich sollte ein Ressourcenzimmer innerhalb der Schule eingerichtet werden, um die individuellen Talente der Kinder zu fördern und die unterschiedlichen Herkunftskulturen und -sprachen verstärkt miteinzubeziehen.

Dies geschieht beispielsweise durch spezielle Angebote wie den „Matheclub“, „Leseclub“, die „Zeitungsmacher“ oder den „Projektnachmittag“. Diese Angebote können von allen Kindern genutzt werden, ausserhalb oder auf Anfrage der Lehrperson während der Unterrichtszeit. Im Klassenzimmer finden andere individuelle Angebote statt, die mit der Leiterin vom Ressourcenzimmer abgestimmt sind. Alle Aktivitäten halten die Kinder in ihrem eigenen Talentportfolio fest.

Alle diese Massnahmen sollen den individuellen Bildungsweg jedes Kindes optimieren.

\section{2. «Es braucht ein ganzes Dorf, um ein Kind zu erziehen.» (Afrikanisches Sprichwort)}

Als schulzentrierte Bildungslandschaft (Jacobs Foundation, 2015), in der die Schule den Knotenpunkt der Bildungslandschaft bildet und die weiteren ausserschulischen Akteure mit sich vernetzt, ging es in einem ersten Schritt darum, das Bildungsverständnis von Lehr- und Fachpersonen Tagesstrukturen innerhalb der Schule abzustimmen.

Deshalb war es für uns vorerst wichtig, innerbetrieblich die beiden Bereiche „Unterricht - Tagesstruktur“ zusammenzuführen, bevor wir nach aussen treten konnten. Denn das längerfristige Ziel lässt sich durch das afrikanische Sprichwort veranschaulichen: „Es braucht ein ganzes Dorf, um ein Kind zu erziehen“.

Non-formale Bildung nicht als Konkurrenz, sondern als Ergänzung anzusehen, bedeutet anzuerkennen, dass die Schule kein Bildungsmonopol besitzt. Die Zusammenarbeit vieler an der Bildung beteiligten Institutionen, so eines der Projektziele, soll die Bildungschancen vergrößern. Der ganzheitliche Blick auf das Kind ermöglicht es, dessen Stärken und Schwächen besser zu erkennen und es gezielter zu fördern.

Beispiele der Zusammenarbeit sind: 
- Zweimal jährlich tauschen sich die Fachpersonen Tagesstrukturen mit den Lehrpersonen intensiv über jedes Kind aus, bevor die Beurteilung im Zeugnis verschriftlicht wird.

- Freiwillige des Jugendrotkreuzes (JRK) ${ }^{1}$ arbeiten an kleinen Forschungsprojekten mit Kindern in der Freizeit. Die Lehrperson und die Eltern erhalten einmal pro Semester eine Rückmeldung zur Arbeit des Kindes.

Seit einigen Jahren ist die Zusammenarbeit in multiprofessionellen Teams in vielen Schulen verbreitet. In multiprofessionellen Teams arbeiten Lehrpersonen, Heilpädagoginnen und Heilpädagogen, Logopädinnen und Logopäden, Psychomotorikerinnen und Psychomotoriker, Schulsozialarbeitende und Fachpersonen Tagesstrukturen. Allerdings haben nicht alle Berufsgruppen gleiche Arbeitsbedingungen, was immer wieder zu Diskussionen unter den Mitarbeitenden führt. Aus meiner Erfahrung als Schulleiterin geht es allerdings in erster Linie darum, den Blick differenziert und breit auf die optimale Entwicklung des Kindes zu richten. Ich beobachte, wie alle Professionen voneinander profitieren und lernen können.

Beispielsweise informiert die Logopädin das Team über die diagnostizierte Legasthenie eines Kindes.

- Sie beschreibt die Symptome und den therapeutischen Ansatz.

- Die Heilpädagogin übernimmt die Lernzielformulierung im Fach Deutsch.

- Die Lehrperson erfährt, mit welchen Methoden sie den Nachteil des Kindes im Unterricht ausgleichen kann.

- Die Sozialarbeiterin spricht mit den Eltern, die das Kind in die extern gelegene Therapie bringen müssen.

Dieses und weitere Fallbeispiele zeigen auf, wie Teamwork im modernen Bildungsverständnis unverzichtbar und nötig ist.

Gelungene Kooperation hilft dem Kind, sein Wohlbefinden zu steigern, und ermöglicht es ihm, sich in seiner Lebenswelt zu bewegen (Kreis et al., 2016).

\section{Unterricht und Sozialpädagogik}

Wie meine bisherige Erfahrung als Schulleiterin zeigt, baut die Vernetzung der beiden Bereiche Unterricht und Tagesstruktur (=Freizeit) auf Vertrauen untereinander und offene Kommunikation. Das Bildungsziel aller Beteiligten in der Schule ist, die Eigenverantwortung jedes einzelnen Kindes zu stärken

1 Das Schweizerische Rote Kreuz vereinigt wie kein anderes Hilfswerk eine Vielfalt von Stärken in den Bereichen Gesundheit, Integration und Rettung unter einem Dach. 
und es somit zu befähigen, selbständig zu werden. Dies beschreibt die PS Thierstein in ihrem pädagogischen Leitbild von 2009. Dies bedeutet Lebenskompetenz zu gewinnen, die im Lehrplan 21 (EDBS, 2016) ${ }^{2}$ folgendermassen beschrieben wird:

„Personale und soziale Kompetenzen befähigen das Kind zur Selbstreflexion, Selbständigkeit, Eigenständigkeit, zur Kooperations- und Konfliktfähigkeit und fördern den Umgang mit Vielfalt.“ (EDBS, 2016, S. 11ff.)

Hierzu fördern Fachpersonen Tagesstrukturen dezidiert die Lebenskompetenzen des Kindes (Handbuch Tagesstrukturen der Volksschulen im Kanton Basel-Stadt, 2013, S. 106; standortspezifisches Förderkonzept PS Thierstein, 2014, S. 18), indem alltägliche Handlungen geübt und reflektiert werden, Gruppenprozesse angeleitet und besprochen und Konfliktsituationen 1:1 mit Einbezug aller Beteiligten angegangen werden.

- Die Kinder lernen Tischmanieren, die in unserer Gesellschaft normiert sind.

- Die Kinder erfahren, wie Körperhygiene anzuwenden ist.

- Die Kinder halten sich selbständig an einfache Abläufe (Hausaufgaben machen, Tische putzen, Boden wischen etc.).

- In einfachen Konfliktsituationen wird den Kindern Raum und Zeit zur selbständigen Lösung angeboten.

\subsection{Kooperation zwischen Lehrpersonen und Fachpersonen Tagesstrukturen}

Die Kooperation zwischen Lehrpersonen und Fachpersonen Tagesstrukturen hat im Rahmen der Einführung der Bildungslandschaft zugenommen. Das veränderte Bildungsverständnis (,lernen passiert überall und immer“") hat da$\mathrm{zu}$ geführt, dass beide Seiten sich bewusster wahrnehmen und voneinander hören. Dazu gehören fix installierte Austauschgefässe, aber auch „Türschwellen"-Gespräche, die es allen ermöglichen, ein breiteres und umfassenderes Bild von einem Kind zu bekommen.

Im Folgenden skizziere ich vier gelungene Kooperationsgefässe und veranschauliche diese an Beispielen. Untenstehend folgen nun Beispiele, wie diese Kooperation konkret aussieht:

\section{Talentportfolio}

Jedes Kind führt ab dem 1. Kindergarten bis zum Ende der Primarschulzeit (während acht Jahren) ein Talentportfolio. Die Fachpersonen Tagesstrukturen

2 Aus: Lehrplan 21 „Überfachliche Kompetenzen; Einleitung“ 
motivieren die Kinder, die während der Freizeit geleisteten Arbeiten ins Portfolio einzufügen. Während der Projekttage der Tagesstrukturen produzierte eine Gruppe von Mädchen der 6. Klasse einen Film von A-Z, was sie im Portfolio festhielten.

Da das Kind seine Stärken darin erfasst, lernt es auf seine Fähigkeiten zu vertrauen und diese weiterzuentwickeln.

Die Lehrperson und Fachpersonen Tagesstrukturen leiten die Kinder an, das Portfolio zu führen und Erfolge zu erfassen und zu reflektieren. Das Talentportfolio dient dann als Diskussionsgrundlage für Einzelgespräche mit dem Kind und für Elterngespräche. Eltern sollen den Wert jeder geleisteten Arbeit ihres Kindes wertschätzen können.

\section{Lernziele}

Die individuellen Lernziele werden von der Lehrperson mit jedem Kind einzeln besprochen. Die Fachpersonen Tagesstrukturen werden einbezogen und die Ziele sind unter allen Fachpersonen Tagesstrukturen im Schulhaus bekannt. Jedes Kind legt mit der Lehrperson auch ein persönliches Lernziel fest. Dieses Ziel teilt es ,seiner“ Fachperson Tagesstrukturen mit, welche zugleich die Bezugsperson ist. Häufig sind dies Ziele, die das soziale Verhalten betreffen.

Wie z.B. „Ich beende einen Streit immer per Handschlag.“ oder „Ich erledige die Hausaufgaben, bevor ich mit dem freien Spiel beginne."

Bei komplexeren Zielformulierungen arbeiten alle Beteiligten mit dem Kind an seinen Zielen, dabei können verschiedene Teilbereiche von verschiedenen Personen abgedeckt werden. Z.B. „Ich unterscheide zwischen guten und schlechten Geheimnissen.“ oder „Ich halte mein Material in Ordnung und kontrolliere es einmal pro Woche."

\section{Qualitätsgruppen}

In den Qualitätsgruppen (Q-Gruppen) arbeiten Lehrpersonen mit Heilpädagoginnen, Lega-Therapeutinnen, Logopädinnen und Fachpersonen Betreuung zusammen. Die Wahl des Themas liegt bei der Gruppe selbst. Jede Gruppe nützt selbstverständlich die verschiedenen Blickwinkel, die eingebracht werden können.

Beispielsweise wird an folgenden Themen der Q-Gruppen gearbeitet:

- Hausaufgabenbetreuung und Lerncoaching

- Bewegungsförderung und Gesundheit

- Klassen-Tagesstruktur-Rat

- Resilienz fördern bei Kindern 
Die Gruppen formulieren nach den Diskussionen Ziele. Sie legen fest, was es braucht, um diese Ziele zu erreichen und woran man erkennt, ob ein Ziel erreicht wurde.

Am Beispiel des Klassenrates / Schülerparlaments, das aktuell bearbeitet wird, lässt sich dies verdeutlichen:

- Die Gruppe will mittelfristig in allen Klassen einen Klassenrat installieren, längerfristig ein „Schulparlament“ einrichten.

- Sie erkennt, dass die politische Bildung ein Thema ist, das untrennbar ist von der Thematik „Klassenrat / Schulparlament“.

- Diese Erkenntnisse bringt die Gruppe in die Schulkonferenz, um abzufragen, ob der Wille vorhanden ist, weiter in die Thematik einzutauchen.

- Die Lehrpersonen und Fachpersonen Tagesstrukturen besprechen das Anliegen und geben weitere Inputs, die die Gruppe wieder aufnimmt.

\section{Leitungsebene}

Die Schulleitung und die Tagesstrukturleitung arbeiten eng zusammen und erkennen die Wichtigkeit der beiden Bereiche füreinander an. Dies wirkt sich auf die Bereitschaft der Mitarbeitenden aus, sich untereinander zu vernetzen, auch wenn dies Mehraufwand bedeutet.

Die Fachperson Tagesstrukturen, welche zugleich die Bezugsperson ist, und die Lehrperson treffen sich, um jedes einzelne Kind zu besprechen. Dabei werden die lernrelevanten Aspekte in einem internen Formular, das von beiden vorab ausgefüllt wird, festgehalten. Die Bezugsperson nimmt bei Bedarf an Elterngesprächen teil und tauscht sich in einer gemischten Intervisionsgruppe aus.

Gleichzeitig ist dieser Mehraufwand für das gelingende Aufwachsen der Heranwachsenden nötig und Ressourcen sind hierzu zur Verfügung zu stellen.

Die Schulen im Kanton Basel-Stadt haben sich als Ziel gesetzt, die ,Schule als Lebenswelt" (FHNW PH und EDBS, 2015, S. 6) zu verstehen. Dies bleibt eine Herausforderung innerhalb des Betriebes, da der Weg zu einem gemeinsamen Bildungsverständnis trotz allem steinig ist. In Bildungsdebatten werden beispielsweise Fachpersonen Tagesstrukturen nicht als gleichwertige Partner für die Lehrpersonen verstanden, was eklatante Lohnunterschiede zwischen den Berufsgruppen unterstreichen. Der einzelnen Schule obliegt es nun, diese Unterschiede mit fairen Bedingungen am Standort bestmöglich auszugleichen. Beispielsweise finden Austauschgespräche während der bezahlten Arbeitszeit der Fachpersonen Tagesstrukturen statt. Die Stellvertretung im Betrieb übernehmen Lehrpersonen in ihrer unterrichtsfreien Zeit.

Um die Herausforderung der „Schule als Lebenswelt“ noch besser zu meistern, ist die Vernetzung und Zusammenarbeit mit Bildungsakteuren ausserhalb der Schule unabdingbar. 


\subsection{Ausserschulische Vernetzung und Angebote}

Als ,schulzentrierte Bildungslandschaft“ müssen wir Kooperationen suchen, die einen Gewinn für alle Beteiligten darstellen, insbesondere aber für das Kind.

$\mathrm{Zu}$ den zentralen Zielen dieser Vernetzung zählen folgende vier:

- Kinder haben bessere Bildungschancen

- Unterstützung der Eltern, Stärkung der Erziehungskompetenz

- Fördern der Selbständigkeit des Kindes

- Netzwerk „Bildungslandschaft“ ermöglicht wertvollen Erfahrungsaustausch untereinander und bringt Wissensgewinn

Die Bildungspartner, mit denen wir über die letzten vier Jahre Kooperationen festigen konnten, sind soziale und pädagogische Einrichtungen, die für und mit Kindern arbeiten. Namentlich sind folgende Angebotsinstitutionen zu nennen: Stadtbibliothek GGG, Forschungsclub Jugend Rotkreuz, FaZ (Familienzentrum Gundeli) und Robi-Spiel-Aktionen, die im Folgenden weiter ausgeführt werden.

Jede dieser Institutionen ermöglicht professionelle Angebote, bei denen das Kind seinen Wissenshorizont oder sein Können erweitert und somit sein Selbstvertrauen stärkt. Die Angebote sind kostenfrei und werden von Fachpersonen unterschiedlicher Arbeitsfelder angeboten. Zum Beispiel bietet die Stadtbibliothek GGG mittwochnachmittags unter der Anleitung einer Sozialpädagogin an, eigene Texte oder Aufsätze zu schreiben, Bilder dazu zu malen und dies bei einem speziellen Event auszustellen. Der Forschungsclub Jugend Rotkreuz hat eigene Versuchsanlagen entworfen, mit denen Kinder selbständig in verschiedenen naturwissenschaftlichen Bereichen ,forschen und experimentieren" können. Das FaZ bietet Bastelkurse an, in denen die Kinder explizit in der deutschen Sprache gestärkt werden. Die Robi-Spiel-Aktionen ermöglichen ein Freizeitangebot am Nachmittag mit Kletterwand und weiteren Spielmöglichkeiten, zu denen die Kinder angeleitet werden.

Diese Angebote stehen beispielhaft für die Zusammenarbeit mit den Netzwerkpartnern im Freizeitbereich, die den schulischen Auftrag an formaler Bildung miterfüllen und gleichzeitig im Bereich der non-formalen Bildung wertvolle Beiträge leisten.

Die Vielfalt der Angebote ermöglicht es jedem Kind, seine Freizeit nach seinen Möglichkeiten zu gestalten. Kinder, die nicht in der Tagesstruktur sind, nützen diese Angebote stärker als Kinder mit Tagesstruktur. Durch die verstärkte Kooperation mit den ausserschulischen Partnern konnten vermehrt Kinder durch die Lehrpersonen in die Angebote vermittelt werden, wenn z.B. die Eltern dazu nicht in der Lage waren. 


\subsection{Soziale Arbeit im Schulhaus - Gelingensbedingungen}

All diese guten Beispiele der Vernetzung basieren auf pädagogischem Konsens. Die optimale Förderung jedes einzelnen Kindes steht im Zentrum.

Oft können Kinder aber nicht an diesen Angeboten teilnehmen, weil die Eltern sie nicht zu einem Angebot begleiten, sie gar nicht anmelden oder bei Mädchen mit islamischem Glauben aus religiösen Motiven die Teilnahme nicht erlauben.

Die Sozialarbeit im Schulhaus leistet hier wertvolle Arbeit, indem sie bei Konflikten mit Eltern im Bereich „Freizeitgestaltung“ interveniert. Sie unterstützt die Schulleitung und die Lehrpersonen in Elternarbeit, bespricht sich mit den Fachpersonen Tagesstrukturen und begleitet Kinder in schwierigen Situationen (z.B. Mobbing, Prüfungsängste).

Die Sozialarbeit begleitet auch ganze Gruppen, die einen problematischen Umgang haben, in speziellen Unterrichtsituationen.

Zum Beispiel beschwert sich eine 5. Klasse über ihre Fachlehrerin beim Klassenlehrer, der in einen Interessenskonflikt gerät. Die Sozialarbeit spricht mit der Kindergruppe und mit den Lehrpersonen und vermittelt unter allen Beteiligten.

Der Fokus kann aber auch auf einem einzelnen Kind liegen, das in der Klasse Hilfe braucht, um sich zu behaupten. Die Sozialarbeit nimmt an Unterrichtssequenzen teil, in denen sich die Probleme des Kindes offenbaren. Für die Lehrperson bringt es Entlastung, für das Kind ist es eine Hilfestellung.

Die bisherigen Erfahrungen an der Thiersteiner Schule zeigen, dass Soziale Arbeit im Unterricht wertvoll und wichtig ist. Es braucht die Offenheit aller Beteiligten, um neben heilpädagogischen Massnahmen auch soziale Arbeit im Klassenzimmer (=Unterricht) zu nutzen (und zu „dulden“).

Zusätzlich braucht es Strukturen, die eine Zusammenarbeit fördern und erleichtern:

- eine gleiche „Sprache“, um Ziele zu formulieren

- $\quad$ strukturelle Anpassung, z.B. gleiche Formulare, um den Lernstand eines Kindes zu erfassen

- Zeitgefässe für den Austausch unter den Fachpersonen

- Zugriff auf alle Daten (elektronische Plattform), die es erlauben, an der ganzheitlichen Entwicklung eines Kindes mitzuarbeiten.

Gleichzeitig wäre hier ein Bildungsauftrag auf Seiten der Fachpersonen der Sozialen Arbeit und Tagesstrukturen nötig, den es noch nicht gibt, aber der wichtige Arbeitsorientierungen in der Kooperation geben könnte. 


\subsection{Soziale Arbeit im Unterricht-konkretes Beispiel}

Die Begleitung durch einen Sozialpädagogen in einer ersten Klasse in unserer Schule im letzten Jahr war ein grosser Gewinn für alle Beteiligten. Der Einsatz wurde nötig aufgrund einer Diagnose eines Kindes, das an AutismusSpektrum-Störung litt. Der Sozialpädagoge war für dieses Kind verantwortlich, übernahm aber auch viel Verantwortung für den Rest der Klasse. Die Zusammenarbeit wurde beidseitig als äusserst spannend und wertvoll empfunden. Die unterschiedlichen pädagogischen Blickwinkel ermöglichten es dem Team, das betroffene Kind, aber auch andere Kinder der Klasse ganzheitlicher zu betrachten und somit gezielter und spezifischer zu fördern.

An der Thiersteiner Schule würden wir uns vermehrt Soziale Arbeit im Unterricht wünschen. Ein gutes Beispiel haben wir erlebt, weitere folgen hoffentlich.

\section{Fazit}

Mit dem Aufbau einer Bildungslandschaft versucht die Primarschule Thierstein, allen Kindern einen optimalen Bildungsweg zu ermöglichen und jedes einzelne Kind in seinen Stärken zu stärken. Die „Schule als Lebensraum“ ist der Ort, an dem formale Bildung stattfindet, an dem aber der Zugang zu nonformaler Bildung ebenso gefördert wird.

Innerhalb der Schule können die Kinder Angebote nutzen, die sie in ihren individuellen Begabungen fördern. Dazu hat die Schule ein Ressourcenzimmer eingerichtet, das beispielsweise $\mathrm{zu}$ allen naturwissenschaftlichen Themen Material bietet.

Die Zusammenarbeit zwischen den Lehrpersonen, den Fachpersonen Tagesstrukturen und der Sozialarbeiterin ist institutionalisiert, braucht aber noch mehr strukturelle Anpassungen, um noch optimaler zu wirken.

Mit einer bisherigen Erfahrung eines Sozialpädagogen im Unterricht möchte sich die Primarschule Thierstein auf den Weg machen, um Soziale Arbeit vermehrt im Unterricht einbauen zu können. Dazu braucht es aber wiederum strukturelle Anpassungen.

Die Soziale Arbeit in der Primarschule Thierstein wäre eine wichtige Unterstützung, damit Lehrpersonen ihren Lehrauftrag erfüllen können und jedes Kind eine optimale Förderung erfahren könnte.

Die Herausforderungen einer sehr heterogenen Schülerschaft und der Auftrag der vollen Integration aller Kinder sind zukünftig ohne Unterstützung durch Fachpersonen Soziale Arbeit aus unserer Sicht nicht mehr leistbar. 


\section{Literatur}

Erziehungsdepartement des Kantons Basel-Stadt (EDBS) - Volksschulen (2016). Lehrplan 21 - Stand 29.02.2016 (Gesamtausgabe). Verfügbar unter: http://bs.lehrplan.ch/container/BS_DE_Gesamtausgabe.pdf [Zugriff: 21.12.2016].

Fachhochschule Nordwestschweiz Pädagogische Hochschule (FHNW PH) \& Erziehungsdepartement des Kantons Basel-Stadt (EDBS) - Volksschulen (2015). Tagesstrukturen. Orientierungsraster für die Schulentwicklung und Schulevaluation an den Volksschulen des Kantons Basel-Stadt. Verfügbar unter: https://www.edubs.ch/schulentwicklung/link/tagesstrukturen [Zugriff: 08.10.2016].

Jacobs Foundation (2015). Bildungslandschaften Schweiz. 22 Netzwerke für mehr Bildungsgerechtigkeit. Zürich. Verfügbar unter:

http://bildungslandschaften.ch/wp-content/uploads/2016/03/2015_22-

Netzwerke_Bildungslandschaften.pdf.pdf [Zugriff: 08.03.2018].

Kreis, Annelies; Wick, Jeannette; Kosorok Labhart, Carmen (Hg.) (2016). Kooperation im Kontext schulischer Heterogenität. 1. Auflage, neue Ausgabe. Münster: Waxmann.

Meier Magistretti, C. \& Walter-Laager, C. (2016). Kriterien wirksamer Praxis in der frühen Förderung: Evidenzbasierte Gestaltung von Angeboten der frühen Förderung mit einem speziellen Fokus auf Kinder aus sozial benachteiligten Familien. Leitfaden. Bern: Bundesamt für Sozialversicherungen.

Statistisches Amt Basel-Stadt (2017a). Bildungsniveau. Verfügbar unter: http://www.statistik.bs.ch/zahlen/tabellen/15-bildungwissenschaft/bildungsniveau.html [Zugriff: 08.03.2018].

Statistisches Amt Basel-Stadt (2017b). Ausländeranteil. Verfügbar unter: http://www.statistik.bs.ch/zahlen/tabellen/1-bevoelkerung/bestand-struktur.html [Zugriff: 08.03.2018].

Züchner, Ivo (2013). Formale, non-formale und informelle Bildung in der Ganztagsschule. In: Jugendhilfe 51 (1), S. 26-35. 


\section{Autorinnen und Autoren}

Simone Ambord ist wissenschaftliche Mitarbeiterin an der Pädagogischen Hochschule Bern (PHBern). Im Rahmen des SNF-Projekts „Kooperationsformen und Nutzungsstrukturen in der Schulsozialarbeit" (www.phbern.ch/schulsozialarbeit) doktoriert sie am Institut für Soziologie der Universität Bern. Ihre Forschungsschwerpunkte sind interprofessionelle Kooperationsforschung, empirische Sozialforschung und Vertrauensforschung.

E-Mail: simone.ambord@phbern.ch

Simon Benz, Student Bachelor in Sozialer Arbeit an der Zürcher Hochschule für Angewandte Wissenschaften (ZHAW). Während seines Studiums arbeitet Simon Benz als Mitarbeiter in Ausbildung im Betreuungsbereich einer Tagesschule der Stadt Zürich.

E-Mail: benzsim2@students.zhaw.ch

Martin Biebricher, Diplom-Pädagoge (Univ.) und Diplom-Sozialarbeiter (FH), ist Dozent für Theorien und Methoden der Sozialen Arbeit am Institut für Kindheit, Jugend und Familie der Zürcher Hochschule für Angewandte Wissenschaften (ZHAW), Departement Soziale Arbeit. Zu seinen Arbeitsschwerpunkten gehören die Themenfelder Theorie- und Methodendiskurse in der Sozialen Arbeit, Kinder- und Jugendhilfe (insb. Kinder- und Jugendarbeit), Evaluation/Qualitätsentwicklung in der Sozialen Arbeit und Geschichte der Sozialen Arbeit.

E-Mail: martin.biebricher@zhaw.ch

Monique Brunner ist wissenschaftliche Mitarbeiterin an der Pädagogischen Hochschule Bern (PHBern). Im Rahmen des SNF-Projekts „Kooperationsformen und Nutzungsstrukturen in der Schulsozialarbeit" (www.phbern.ch/schulsozialarbeit) doktoriert sie am Institut für Soziologie der Universität Bern. Ihre Forschungsschwerpunkte sind interprofessionelle Kooperationsforschung, empirische Sozialforschung und Geschlechterforschung.

E-Mail: monique.brunner@phbern.ch

Markus Buholzer, MAS Schulmanagement, Rektor der Volksschule Kriens. Er setzt auf multisystemische Kooperation zwecks Stärkung der Bindung zwischen dem Kind 
und seinen Vertrauenspersonen und versteht diese Bindung als Voraussetzung für Lernprozesse.

E-Mail: markus.buholzer@kriens.ch

Esther Bussmann, Sozialarbeitende, Dozentin, Zentrum Lehre der Zürcher Hochschule für Angewandte Wissenschaft (ZHAW), Departement Soziale Arbeit. Ihre inhaltlichen Schwerpunkte sind die Praxisausbildung, Interprofessionelle/Interdisziplinäre Kooperation und Internationale Perspektiven.

E-Mail: Esther.Bussmann@zhaw.ch

Emanuela Chiapparini, Dr. phil., ist Erziehungswissenschaftlerin und Dozentin am Institut für Kindheit, Jugend und Familie an der Zürcher Hochschule für Angewandte Wissenschaften (ZHAW), Soziale Arbeit. Gegenwärtig leitet sie am Standort ZHAW Soziale Arbeit das SNF-Projekt zu pädagogischen Zuständigkeiten an Tagesschulen (www.zhaw.ch/sozialearbeit/auster). Weitere Forschungsschwerpunkte sind ausserschulische Bildung, Peergroups, soziale Ungleichheit, Civic Engagement und User Involvement.

E-Mail: Emanuela.Chiapparini@zhaw.ch

Marco Dalcher ist Sozialpädagoge FH und hat ein Master Studium (Executive Master of Business Administration) an der Hochschule für Wirtschaft in Basel absolviert. Seit 2014 arbeitet er beim Erziehungsdepartement Basel-Stadt auf der Fachstelle Tagesstrukturen. Innerhalb seiner Tätigkeit war er schwerpunktmässig mit dem Aufbau der Tagesstrukturen an der Sekundarschule des Kantons Basel-Stadt beauftragt.

E-Mail: Marco.Dalcher@bs.ch

Ueli Hostettler, Prof. Dr. habil., ist Dozent und Leiter des Forschungsschwerpunkts „Governance im System Schule“ an der Pädagogischen Hochschule Bern (PHBern) und verantwortlicher Leiter des SNF-Projekts „Kooperationsformen und Nutzungsstrukturen in der Schulsozialarbeit" (www.phbern.ch/schulsozialarbeit). Daneben leitet er die Forschungsgruppe „Prison Research Group“ an der Universität Bern (prisonresearch.ch).

E-Mail: ueli.hostettler@phbern.ch

Joël Humbert-Droz arbeitet als Sozialpädagoge und Berufsintegrationscoach für die Schule in Kleingruppen und für die Sekundarschule Dielsdorf ZH. Er hat das Studium der Sozialpädagogik und die Weiterbildung zum Berufsintegrationscoach an der FHNW in Olten absolviert. Zu seiner Hauptaufgabe gehört es, Jugendliche beim gesamten Berufswahl- und Ausbildungsprozess individuell zu unterstützen und zu begleiten.

E-Mail: j.humbert@kgs-dielsdorf.ch

Andreas Jud, Prof. Dr. leitet den Lehrstuhl Epidemiologie und Verlaufsforschung im Kinderschutz an der Kinder- und Jugendpsychiatrie/Psychotherapie des Universitätsklinikums Ulm. Auch als Dozent/Projektleiter an der Hochschule Luzern - Soziale 
Arbeit ist er seit mehreren Jahren in der interdisziplinären Forschung zum Kinderschutz tätig. Seine Studien betreffen das Ausmass von Kindeswohlgefährdung und Entscheidungsprozesse im institutionalisierten Kinderschutz. Seine internationale Karriere umfasst ausserdem einen Postdoc-Aufenthalt 2011 an der McGill University in Montreal, Kanada, und die Mitherausgeberschaft des Peer-Review-Journals „Child Abuse \& Neglect“".

E-Mail: andreas.jud@hslu.ch

Christa Kappler, Dr. phil., hat an der Universität Zürich Pädagogik und Soziologie studiert und promovierte 2013 in Erziehungswissenschaften an der Universität Bern. Seit 2009 arbeitet sie als wissenschaftliche Mitarbeiterin und Dozentin in der Abteilung Forschung und Entwicklung der Pädagogischen Hochschule Zürich. Ihre inhaltlichen Schwerpunkte liegen in den Bereichen Ganztagsbildung, Studien- und Berufswahlprozesse, Gender und Diversity sowie Methoden der qualitativen Sozialforschung.

E-Mail: christa.kappler@phzh.ch

Jacques Kottelat, Sozialpädogoge (Diplom eesp Lausanne und Master in Erziehungswissenschaften Uni Genf), arbeitet als Schulsozialarbeiter in Biel. Als Lehrbeauftragter unterrichtet er an den Fachhochschulen für Soziale Arbeit in Genf, Lausanne, Fribourg und Sierre, daneben an der Pädagogischen Hochschule BEJUNE (Bern, Jura Neuenburg). Seine Forschungsschwerpunkte sind insbesondere schulische Inklusion, Team Learning und Kooperation.

E-Mail: j.kottelat@bluewin.ch

David Lätsch, Dr., ist Dozent (Prof. FH) und Forschungsleiter im Departement Soziale Arbeit der Berner Fachhochschule. In seiner Arbeit setzt er sich mit Ursachen, Formen und Folgen von Kindeswohlgefährdungen auseinander. Von besonderem Interesse ist dabei die Frage, wie Institutionen und Akteure des Bildungs- und Gesundheitswesens, der Kinder- und Jugendhilfe und insbesondere des Kindesschutzes dem Auftreten von Kindeswohlgefährdungen wirkungsvoll vorbeugen und sie in ihren Folgen eindämmen können.

E-Mail: david.laetsch@bfh.ch

Claudia Mühlebach, éducatrice sociale, Master en direction et stratégie d'institutions éducatives, sociales et socio-sanitaires, Directrice d'une institution pré- et d'une parascolaire dans le canton de Vaud, Intervenante à l'école supérieur en éducation (esede) à Lausanne, Membre du comité de Pro Enfance.

E-Mail: claudia.muhlebach@bluewin.ch

Roger Pfiffner ist Dozent und Projektleiter am Fachbereich für Soziale Arbeit der Berner Fachhochschule. Dort beschäftigt er sich mit Fusionen sozialer Dienste und forscht zu Fragen des Qualitätsmanagements, der Personalentwicklung sowie der interdisziplinären Zusammenarbeit. Er ist Mitantragsteller beim SNF-Forschungsprojekt „Kooperationsformen und Nutzungsstrukturen in der Schulsozialarbeit“.

E-Mail: roger.pfiffner@bfh.ch 
Anna Maria Riedi, Prof. Dr. phil., lehrt und forscht am Institut für Kindheit, Jugend und Familie an der Zürcher Hochschule für Angewandte Wissenschaften (ZHAW), Departement Soziale Arbeit zu den Themen Aufwachsen, Gender, Diversity sowie Soziale Probleme/Soziale Konflikte. Sie ist Vorstandsmitglied der okaj Zürich, kantonale Kinder- und Jugendförderung.

E-Mail: annamaria.riedi@zhaw.ch

Patricia Schuler Braunschweig, Prof. Dr., ist promovierte Erziehungswissenschaftlerin und leitet an der Pädagogischen Hochschule das Zentrum „Professionalisierung und Kompetenzentwicklung“. Sie hat eine Professur zur Professionsforschung unter besonderer Berücksichtigung innovativer Kontexte inne. Ihre inhaltlichen Schwerpunkte liegen in den Bereichen der Ganztagsbildung, des Kompetenzerwerbes und der Messung von überfachlichen Kompetenzen, der Auswahl von Lehramtskandidatinnen und -kandidaten und den Methoden der qualitativen Sozialforschung.

E-Mail: patricia.schuler@phzh.ch

Kadrie Selmani, M.A., studierte an der Universität Zürich Soziologie und Biologie und verfasst gegenwärtig ihre Dissertation in Erziehungswissenschaften an der Universität Zürich. Seit 2016 arbeitet sie als wissenschaftliche Assistentin an der Zürcher Hochschule für Angewandte Wissenschaften (ZHAW), Soziale Arbeit. Ihre inhaltlichen Schwerpunkte liegen in den Bereichen Tagesschule, ausserschulische Bildung, Kindheits- und Wohlbefindensforschung.

E-Mail: kadrie.selmani@zhaw.ch

Mirjam Staub, MA UZH, ist Erziehungswissenschaftlerin, Sozialpädagogin FH und Lehrerin HF für Sozialpädagogik an der Höheren Fachschule Gesundheit und Soziales (HFGS) in Aarau. Sie doktoriert am Lehrstuhl Historische Bildungsforschung und Steuerung des Bildungssystems, Institut für Erziehungswissenschaft der Universität Zürich zum Thema der Geschichte der ausserschulischen Betreuung von Schulkindern im 19. und 20. Jahrhundert (https://www.uzh.ch/blog/ife-hbs/forschungsprojekte/laufend/ kinderhorte/).

E-Mail: Mirjam.Staub@uzh.ch

Madlaina Stauffer, lic. phil., ist Psychologin, arbeitet als wissenschaftliche Mitarbeiterin im Arbeitsschwerpunkt Soziale Interventionen am Departement Soziale Arbeit der Berner Fachhochschule und doktoriert an der Universität Zürich in der Erziehungswissenschaft. An der BFH arbeitet sie derzeit an verschiedenen Projekten zum Thema Kindesschutz, ein Schwerpunkt ihrer bisherigen Tätigkeit lag in der Mitarbeit bei Evaluationen von Präventionsprogrammen an Schulen.

E-Mail: madlaina.stauffer@bfh.ch

Renate Stohler, lic. phil., ist Erziehungswissenschaftlerin und arbeitet als Dozentin und Projektleiterin im Institut für Kindheit, Jugend und Familie der Zürcher Hochschule für Angewandte Wissenschaften (ZHAW), Departement Soziale Arbeit. Ihre 
Arbeits- und Forschungsschwerpunkte sind Soziale Arbeit und Schule sowie Fremdunterbringung in Pflegefamilien und Heimen.

E-Mail: renate.stohler@zhaw.ch

Nina Thieme, Dr. phil., Gastprofessorin für Soziale Arbeit an der Universität Kassel; Forschungs- und Arbeitsschwerpunkte: Kinder- und Jugendhilfe, Professions-, Professionalisierungs- und Professionalitätsforschung Sozialer Arbeit, Bildungsungerechtigkeit, multiprofessionelle Kooperation (in inklusiven Settings), qualitativ-rekonstruktive Sozialforschung.

E-Mail: nina.thieme@uni-kassel.de

Karin Vaneck Hubmann, Schulleiterin an der Primarstufe Thierstein, Basel- Stadt. E-Mail: karin.vaneck@bs.ch

Aldo Venzi, MAS Supervision, Coaching \& Mediation, BSc Soziale Arbeit, lehrt am Institut Sozialmanagement an der Zürcher Hochschule für Angewandte Wissenschaften (ZHAW), Departement Soziale Arbeit. Zu seinen Arbeitsschwerpunkten gehören die Themenfelder Praxisausbildung in der Sozialen Arbeit, Team- und Personalführung, Gesprächsführung, Konfliktmanagement, Gruppendynamik, Supervision und Coaching. Er ist für das Institut Sozialmanagement als Berater im Praxisfeld der Sozialen Arbeit und in verwandten Arbeitsfeldern tätig.

E-Mail: aldo.venzi@zhaw.ch

Hans-Peter von Aarburg, Dr. phil., Ethnologe, forschte und unterrichtete als Professor an der Fachhochschule für Soziale Arbeit in Sierre, dies bis zu seiner Pensionierung Ende 2017. Wohl auch aufgrund seiner Erfahrungen als ehemaliger Sekundarlehrer fokussierte er Schnittstellen zwischen Schule und Sozialer Arbeit. Methodisch orientierte er sich - wie in früheren Forschungen zu Themen der Migration und des Drogenkonsums - an biographischen und zunehmend auch sozialräumlichen Ansätzen.

E-Mail: hp.vonaarburg@bluewin.ch

Marianne Zogmal, Dr. in Erziehungswissenschaften, arbeitet an der Universität Genf als wissenschaftliche Mitarbeiterin. Die Forschungsschwerpunkte sind die Berufsbildung in Arbeitssituationen und ein langjähriges Forschungsprojekt betrifft die Ausbildung der KindererzieherIn HF. Gleichzeitig ist sie als Leiterin einer Kindertagesstätte in der Stadt Genf tätig.

E-Mail: zogmal@sunrise.ch 